\title{
Synthesis of Seven-membered Cross-conjugated Cyclic Trienes by $8 \pi$ Electrocyclic Reaction
}

\author{
Ranmaru Kato ${ }^{\mathrm{a}}$, Hiroki Saito ${ }^{\mathrm{a}}$, Shoko Uda ${ }^{\mathrm{a}}$, Daisuke Domon ${ }^{\mathrm{a}}$, Kazutada Ikeuchi ${ }^{\mathrm{b}}$, Takahiro Suzuki ${ }^{\mathrm{b}}$, \\ and Keiji Tanino*b
}

${ }^{a}$ Graduate School of Chemical Sciences and Engineering, Hokkaido University, Sapporo 060-0810, Japan

${ }^{\mathrm{b}}$ Department of Chemistry, Faculty of Science, Hokkaido University, Sapporo 060-0810, Japan

*Corresponding Author (E-mail: ktanino@sci.hokudai.ac.jp)

\section{Table of Contents}

Supplementary Figures, Schemes and Tables

$\begin{array}{ll}\text { Table S1. Investigation on the effect of different leaving groups } & \text { S2 }\end{array}$

$\begin{array}{ll}\text { Scheme S1. Substrates which could not be prepared } & \text { S2 }\end{array}$

$\begin{array}{ll}\text { Scheme S2. Reaction of a triene having no leaving group } & \text { S3 }\end{array}$

Table S2. Optimization of base for the cyclization of $\mathbf{6 a}$ and $7 \mathbf{a} \quad$ S3

Scheme S3. Intramolecular nitrile aldol reaction under mild silylation conditions, and its $\begin{array}{ll}\text { application to the synthesis of } \mathbf{1 8 b} & \mathrm{S} 4\end{array}$

General Information $\quad$ S5

Experimental and Characterization Details

$\begin{array}{ll}\text { Preparation of } \gamma, \delta \text {-unsaturated esters } & \text { S6 }\end{array}$

$\begin{array}{ll}\text { Preparation of } \alpha, \beta \text {-unsaturated aldehyde S14 } & \text { S7 }\end{array}$

$\begin{array}{ll}\text { Preparation of other } \alpha, \beta \text {-unsaturated aldehydes } & \mathrm{S} 10\end{array}$

General procedures for preparation of trienyl ester (for syntheses of 7a,b, 12a-d, 18a,b, 20a) S12

Preparation of other trienyl esters (for syntheses of 7c, 14, 16, 20b) S21

$\begin{array}{ll}\text { Preparation of phosphate 6a } & \text { S26 }\end{array}$

$\begin{array}{ll}\text { General procedure for preparation of cyclization precursors } & \text { S27 }\end{array}$

$\begin{array}{ll}\text { General procedure for } 8 \pi \text { electrocyclic reaction } & \text { S33 }\end{array}$

$\begin{array}{ll}\text { Structural assignment of 21a by cyclopropanation } & \text { S39 }\end{array}$

$\begin{array}{ll}\text { Transformation of cross-conjugated triene into cycloheptatrienyl cation } & \text { S39 }\end{array}$

$\begin{array}{ll}\text { References } & \text { S41 }\end{array}$

${ }^{1} \mathrm{H}$ and ${ }^{13} \mathrm{C}$ NMR Spectra $\quad$ S42-S153 


\section{Supplementary Figures, Schemes and Tables}

Table S1. Investigation on the effect of different leaving groups

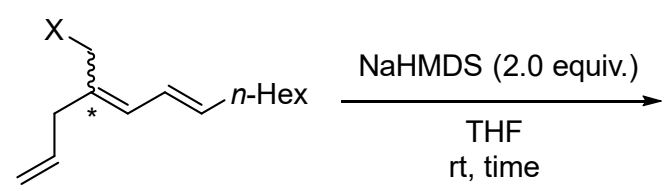

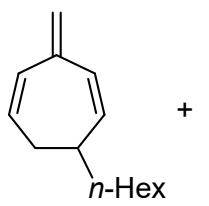

$8 a$

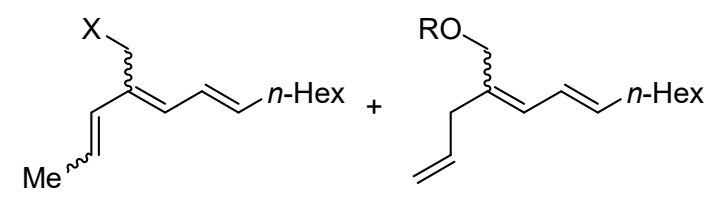

S5
S6 $(R=T M S)$ $5 a(R=H)$

6a, 7a, S1-4 $\left({ }^{*} E / Z=4: 1\right)$<smiles>CCC</smiles>

entry substrate

X time

NMR yield

\begin{tabular}{cclrccccc} 
entry & substrate & \multicolumn{1}{c}{$\mathrm{X}$} & time & $\mathbf{8 a}$ & SM recovery & $\mathbf{S 5}$ & $\mathbf{S 6}$ & $\mathbf{5 a}$ \\
\hline 1 & $\mathbf{6 a}$ & $\mathrm{OP}(=\mathrm{O})(\mathrm{OEt})_{2}$ & $1.5 \mathrm{~h}$ & $69 \%$ & - & - & - & - \\
2 & $\mathbf{7 a}$ & $\mathrm{OC}(=\mathrm{O}) \mathrm{NMe}_{2}$ & $2 \mathrm{~h}$ & $72 \%$ & - & $13 \%$ & - & - \\
3 & $\mathbf{S 1}$ & $\mathrm{OC}(=\mathrm{O}) \mathrm{MMe}$ & $2 \mathrm{~h}$ & $<5 \%$ & - & - & $40 \%$ & $13 \%$ \\
4 & $\mathbf{S 2}$ & $\mathrm{OC}(=\mathrm{O}) \mathrm{t}-\mathrm{Bu}$ & $2 \mathrm{~h}$ & $<10 \%$ & $<10 \%$ & - & $51 \%$ & $26 \%$ \\
5 & $\mathbf{S 3}$ & OC(=O)Ph & $2 \mathrm{~h}$ & - & $23 \%$ & - & $21 \%$ & $11 \%$ \\
6 & $\mathbf{S 4}$ & OTIPS & $2 \mathrm{~h}$ & $<5 \%$ & $>95 \%$ & - & - & - \\
$7^{\mathrm{a}}$ & $\mathbf{S 4}$ & OTIPS & $4 \mathrm{~h}$ & $53 \%{ }^{\mathrm{b}}$ & - & - & - & - \\
\hline
\end{tabular}

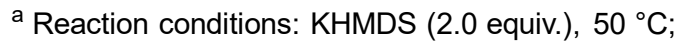

${ }^{\mathrm{b}}$ Byproducts were formed.

Optimization of the leaving group was conducted by using 4-substituted trideca-1,4,6-triene (Table S1). Substrates having various leaving groups (6a, 7a and S1-4) were treated with 2.0 equivalents of NaHMDS at room temperature. From phosphate $6 \mathbf{a}$ and carbamate $7 \mathbf{a}$, the desired product $\mathbf{8 a}$ was obtained in 69\% and 72\% NMR yield respectively (entry 1, 2), while from other precursors, 8a was generated only as a minor product (entry 3-6). In entry 7, treatment of silyl ether S4 with KHMDS at $50{ }^{\circ} \mathrm{C}$ afforded $\mathbf{8 a}$ in $55 \% \mathrm{NMR}$ yield, as an inseparable mixture with tremendous amount of byproduct.

Scheme S1. Substrates which could not be prepared<smiles>C=CCC(=CC=CO)CO</smiles>

$5 \mathbf{a}$<smiles>[R][Ca]/C=C/C=C(/CO)CC=C</smiles>

$5 a$

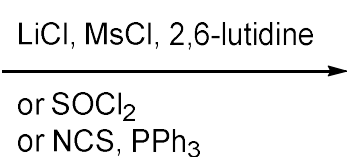
or NCS, $\mathrm{PPh}_{3}$

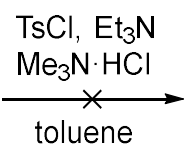

toluene<smiles>[R]C=CC=CC(Cl)C(=C)CC=C</smiles>

S7<smiles>C=CCC(=CC=C[18O])CO[As]</smiles>

s9 
Various methods for chlorination of 5a resulted in formation of desired $\mathbf{S} 7$ along with $\mathrm{S}_{\mathrm{N}} 2^{\prime}$ byproduct S8. By treatment of the resultant crude mixture with NaHMDS in THF, partial conversion of $\mathbf{S 7}$ into cyclized product 8a was observed. We could not obtain a $p$-toluene sulfonyloxyfunctionalized S9 probably due to its instability (Scheme S1).

Scheme S2. Reaction of a triene having no leaving group

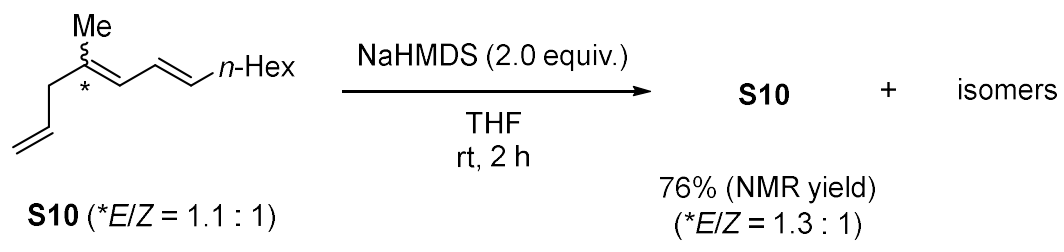

Treatment of triene S10 with 2.0 equivalents of NaHMDS at room temperature resulted in $76 \%$ recovery of starting material (Scheme S2). This result suggests that in the reactions of phosphate $\mathbf{6 a}$ and carbamate $7 \mathbf{a}$ the leaving groups assisted the generation of anionic intermediate by the inductive effect.

Table S2. Optimization of base for the cyclization of $\mathbf{6 a}$ and $7 \mathbf{a}$
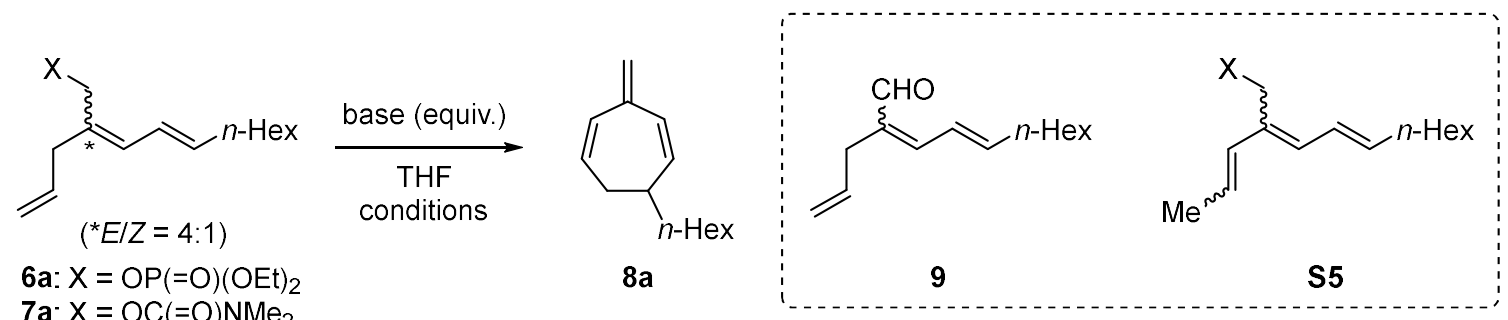

\begin{tabular}{|c|c|c|c|c|c|}
\hline entry & substrate & base & equiv. & conditions & results (NMR yield) \\
\hline 1 & $6 a$ & LDA & 2.0 & rt $0.5 \mathrm{~h}$ & $\mathbf{6 a}(9 \%), \mathbf{9}(53 \%)$ \\
\hline 2 & $6 a$ & LTMP & 2.0 & rt $1.5 \mathrm{~h}$ & $\mathbf{6 a}(9 \%), \mathbf{9}(23 \%)$ \\
\hline 3 & $6 a$ & LHMDS & 2.0 & rt. $1.5 \mathrm{~h}$ & $\mathbf{6 a}(16 \%), \mathbf{8 a}(35 \%)$ \\
\hline 4 & $6 a$ & LHMDS & 2.0 & $\mathrm{rt}, 2 \mathrm{~h} ; 50^{\circ} \mathrm{C}, 1 \mathrm{~h}$ & $8 \mathbf{a}(48 \%)$ \\
\hline 5 & $6 a$ & NaHMDS & 2.0 & $\mathrm{rt} .1 .5 \mathrm{~h}$ & $8 \mathbf{a}(69 \%)$ \\
\hline 6 & $6 a$ & NaHMDS & 1.1 & rt $1.5 \mathrm{~h}$ & $8 \mathbf{a}(59 \%)$ \\
\hline 7 & $6 a$ & KHMDS & 2.0 & rt: $0.5 \mathrm{~h}$ & $8 \mathbf{a}(64 \%)$ \\
\hline 8 & $6 a$ & KHMDS & 1.1 & rt: $0.5 \mathrm{~h}$ & $\mathbf{8 a}(36 \%), \mathbf{S 5}(18 \%)$ \\
\hline 9 & $7 a$ & LDA & 2.0 & rt $0.5 \mathrm{~h}$ & complex mixture \\
\hline 10 & $7 a$ & LTMP & 2.0 & $\mathrm{rt}, 2 \mathrm{~h}$ & complex mixture \\
\hline 11 & $7 a$ & LHMDS & 2.0 & $\mathrm{rt}, 2 \mathrm{~h}$ & $7 \mathbf{a}(>95 \%), 8 \mathbf{a}(<5 \%)$ \\
\hline 12 & $7 a$ & LHMDS & 2.0 & $\mathrm{rt}, 0.5 \mathrm{~h} ; 50^{\circ} \mathrm{C}, 3.5 \mathrm{~h}$ & $7 a(52 \%), 8 a(30 \%)$ \\
\hline 13 & $7 a$ & NaHMDS & 2.0 & $\mathrm{rt}, 2 \mathrm{~h}$ & $8 \mathbf{a}(72 \%), \mathbf{S 5}(13 \%)$ \\
\hline 14 & $7 a$ & KHMDS & 2.0 & $\mathrm{rt}, 2 \mathrm{~h}$ & $8 a(64 \%)$ \\
\hline 15 & $7 a$ & KHMDS & 1.1 & $\mathrm{rt}, 2 \mathrm{~h}$ & $8 \mathbf{a}(55 \%)$ \\
\hline
\end{tabular}


Optimization of base has revealed that use of 2.0 equivalents of NaHMDS or KHMDS was suitable for cyclization of both $\mathbf{6 a}$ and $7 \mathbf{a}$ (Table S2, S3). KHMDS exhibited even higher reactivity than NaHMDS, but formation of inseparable impurities was problematic.

Scheme S3. Intramolecular nitrile aldol reaction under mild silylation conditions, and its application to the synthesis of $\mathbf{1 8 b}$
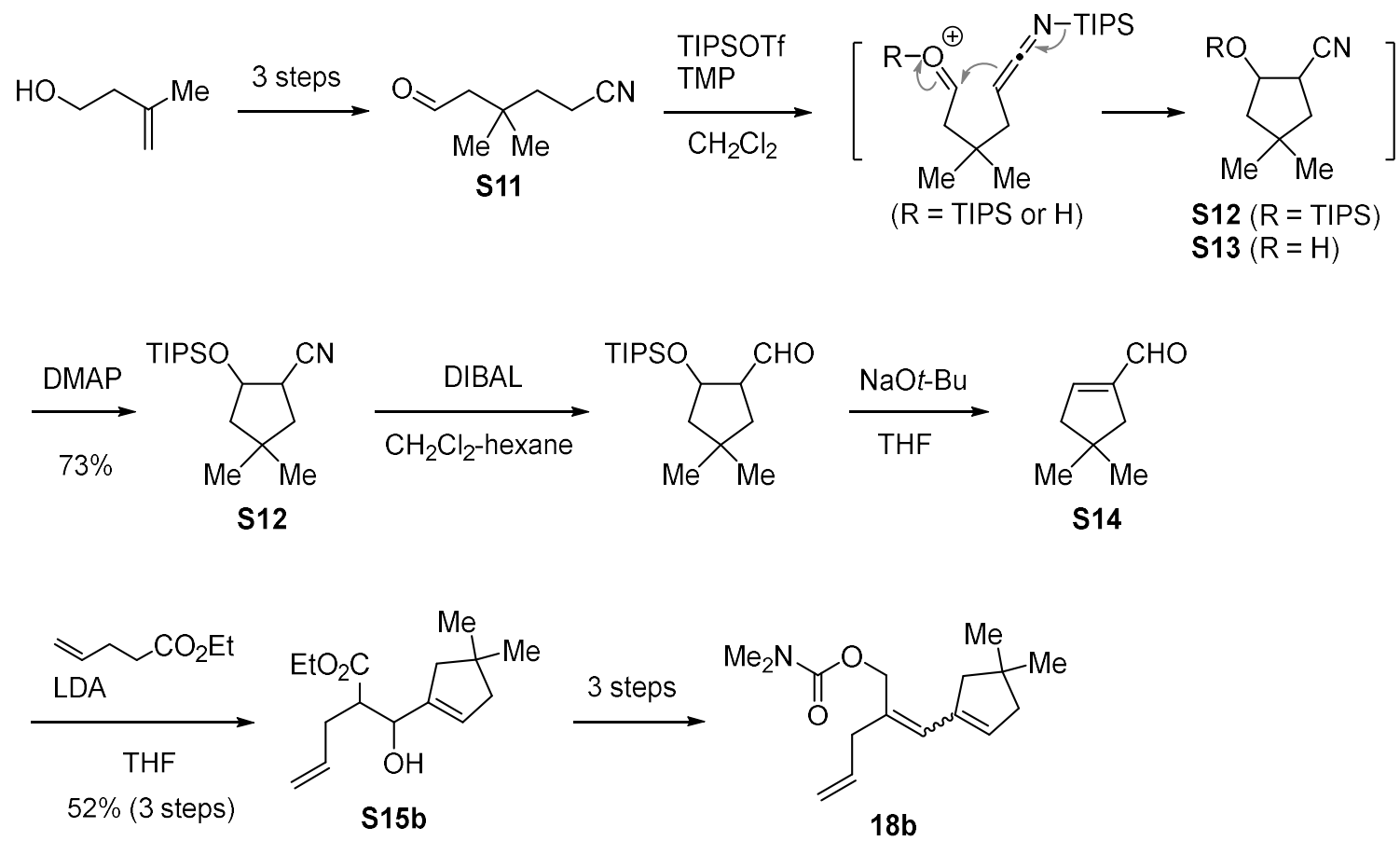

To synthesize cyclization precursor $\mathbf{1 8 b}$, we established a new synthetic route to obtain intermediate S14, which we considered as accessible using our previously reported method (Scheme S3) ${ }^{[1]}$. A cyanoaldehyde S11 was synthesized from 3-methyl-3-butene-1-ol in 3 steps. By treatment of S11 with TIPSOTf and TMP, $N$-silylketeneimine was generated in situ, which cyclized to $\beta$-siloxynitrile S12. However, at the same time $\beta$-hydroxynitrile S13 was generated, thus we then added DMAP to promote silylation of S13. Reduction and $\beta$-elimination of $\mathbf{S 1 2}$ afforded $\alpha, \beta$-unsaturated aldehyde S14, which was then subjected to aldol reaction with ethyl 4-pentenoate to give S15b. S15b was converted to $\mathbf{1 8 b}$ according to general methods. 


\section{General Information}

The reactions were performed using flame-dried glasswares under a positive pressure of argon. Reactions at elevated temperatures were performed under heating in an oil bath. THF and $\mathrm{Et}_{2} \mathrm{O}$ were distilled from sodium benzophenone ketyl. Other anhydrous solvents were purchased from chemical companies. $i-\mathrm{Pr}_{2} \mathrm{NH}, \mathrm{Et}_{2} \mathrm{NH}$ and $\mathrm{Et}_{3} \mathrm{~N}$ were distilled from $\mathrm{CaH}_{2}$ under argon and stored in the presence of $\mathrm{NaOH}$ (pellets). All other reagents were used as received from commercial sources without further purification.

${ }^{1} \mathrm{H}$ NMR spectra were measured using a JEOL ECA-500 $(500 \mathrm{MHz})$ in $\mathrm{CDCl}_{3}\left(\delta_{\mathrm{H}} 7.26\right)$. Chemical shifts are reported in parts per million (ppm), and signal are expressed as broad (br), singlet (s), doublet (d), triplet (t), quartet (q), and multiplet (m). Coupling constants are reported in Hz. ${ }^{13} \mathrm{C}$ NMR spectra were measured using a JEOL ECA-500 $(126 \mathrm{MHz})$ in $\mathrm{CDCl}_{3}\left(\delta_{\mathrm{C}} 77.0\right)$. Highresolution mass spectra (HRMS) were recorded on a JEOL JMS-T100GCV (GC-TOFMS) at the GC-MS \& NMR Laboratory, Faculty of Agriculture, Hokkaido University. Fourier transform infrared (FT-IR) spectra were recorded on a JASCO FT/IR-4100 spectrophotometer. Analytical thin layer chromatography (TLC) was performed using Silica Gel $60 \mathrm{~F}_{254}$ (E. Merck) or Chromatorex NH-TLC Plates (Fuji Silysia Chemical Ltd.), and preparative thin layer chromatography (PTLC) was performed using PLC Silica Gel $60 \mathrm{~F}_{254}$ (E. Merck). Reaction components were visualized by illumination with ultraviolet light $(254 \mathrm{~nm})$ and by staining with $8 \%$ ethanolic phosphomolybdic acid, ceric ammonium molybdate in $10 \%$ sulfuric acid, or basic potassium permanganate aqueous solution. Silica Gel 60N (spherical, neutral, Kanto Chemical Co.) or Chromatorex NH-DM1020 (Fuji Silysia Chemical Ltd.) was used for flash column chromatography. 


\section{Experimental and Characterization Details}

\section{Preparation of $\gamma, \delta$-unsaturated esters}

\section{Ethyl 4-methyl-4-pentenoate}

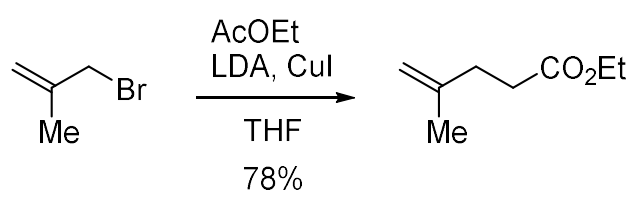

To a solution of $i$ - $\operatorname{Pr}_{2} \mathrm{NH}(1.69 \mathrm{~mL}, 12.0 \mathrm{mmol})$ in THF $(50 \mathrm{~mL})$ was added $n$-BuLi $(2.69 \mathrm{~mol} / \mathrm{L}$ in hexane, $4.46 \mathrm{~mL}, 12.0 \mathrm{mmol}$ ) at $0{ }^{\circ} \mathrm{C}$. After stirring for $30 \mathrm{~min}$, the reaction mixture was cooled down to $-78{ }^{\circ} \mathrm{C}$ and AcOEt $(1.17 \mathrm{~mL}, 12.0 \mathrm{mmol})$ and $\mathrm{CuI}(2.29 \mathrm{~g}, 12.0 \mathrm{mmol})$ were added successively. The reaction mixture was then warmed up to $-30{ }^{\circ} \mathrm{C}$ and stirred for $30 \mathrm{~min}$. To this mixture was added methallyl bromide $(1.02 \mathrm{~mL}, 10.0 \mathrm{mmol})$ and the reaction mixture was stirred for $10 \mathrm{~min}$ at the same temperature. The reaction was quenched with saturated aqueous $\mathrm{NH}_{4} \mathrm{Cl}$ solution $(30 \mathrm{~mL})$. Two layers were separated, and the aqueous layer was extracted with $\mathrm{Et}_{2} \mathrm{O}(20 \mathrm{~mL}$ $\times 3$ ). The combined organic layer was dried over $\mathrm{MgSO}_{4}$, filtered, and concentrated under reduced pressure. The resulting residue was purified by flash column chromatography $\left(\mathrm{SiO}_{2}\right.$, pentane:Et $\mathrm{O}_{2}$ $=20: 1)$ to afford the title compound $(1.11 \mathrm{~g}, 7.81 \mathrm{mmol}, 78 \%$ yield $)$ as a yellow oil. ${ }^{1} \mathrm{H}$ spectral property was identical to that reported ${ }^{[2]}$.

\section{Ethyl 3-(cyclopenten-1-yl)propanoate}

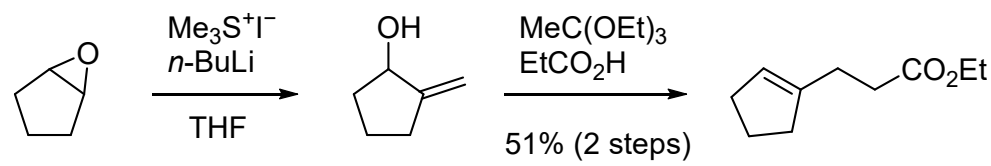

To a solution of trimethylsulfonium iodide $(8.16 \mathrm{~g}, 40.0 \mathrm{mmol})$ in THF $(50 \mathrm{~mL})$ was added $n$-BuLi $(2.64 \mathrm{~mol} / \mathrm{L}$ in hexane, $12.5 \mathrm{~mL}, 33.0 \mathrm{mmol})$ at $-20{ }^{\circ} \mathrm{C}$. After stirring for $30 \mathrm{~min}$, cyclopentene oxide $(867 \mu \mathrm{L}, 10.0 \mathrm{mmol})$ was added to the reaction mixture. The reaction mixture was then warmed up to room temperature over $30 \mathrm{~min}$ and stirred for additional $1.5 \mathrm{~h}$. The reaction was quenched with $\mathrm{H}_{2} \mathrm{O}(30 \mathrm{~mL})$. Two layers were separated, and the aqueous layer was extracted with $\mathrm{Et}_{2} \mathrm{O}(30 \mathrm{~mL} \times$ 3). The combined organic layer was dried over $\mathrm{MgSO}_{4}$, filtered, and concentrated under reduced pressure to give a crude oil.

To a solution of crude alcohol in triethyl orthoacetate $(9.11 \mathrm{~mL}, 50.0 \mathrm{mmol})$ was added propionic acid $(74.1 \mu \mathrm{L}, 1.00 \mathrm{mmol})$, and the reaction mixture was refluxed at $145{ }^{\circ} \mathrm{C}$ for $4 \mathrm{~h}$. The reaction mixture was then cooled down to room temperature and diluted with $\mathrm{Et}_{2} \mathrm{O}(10 \mathrm{~mL}) .1 \mathrm{~N}$ aqueous $\mathrm{HCl}$ solution $(10 \mathrm{~mL})$ was then added to the reaction mixture. After stirring for $1.5 \mathrm{~h}$, two layers 
were separated, and the aqueous layer was extracted with $\mathrm{Et}_{2} \mathrm{O}(10 \mathrm{~mL} \times 3)$. The combined organic layer was dried over $\mathrm{MgSO}_{4}$, filtered, and concentrated under reduced pressure. The resulting residue was purified by flash column chromatography $\left(\mathrm{SiO}_{2}\right.$, hexane:EtOAc $\left.=20: 1\right)$ to afford the title compound ( $859 \mathrm{mg}, 5.11 \mathrm{mmol}, 51 \%$ yield for 2 steps) as a yellow oil. ${ }^{1} \mathrm{H}$ spectral property was identical to that reported ${ }^{[3]}$.

\section{Preparation of $\alpha, \beta$-unsaturated aldehyde S14 (Scheme S3)}

\section{4,4-Dimethyl-6-((triethylsilyl)oxy)hexanenitrile (S16)}

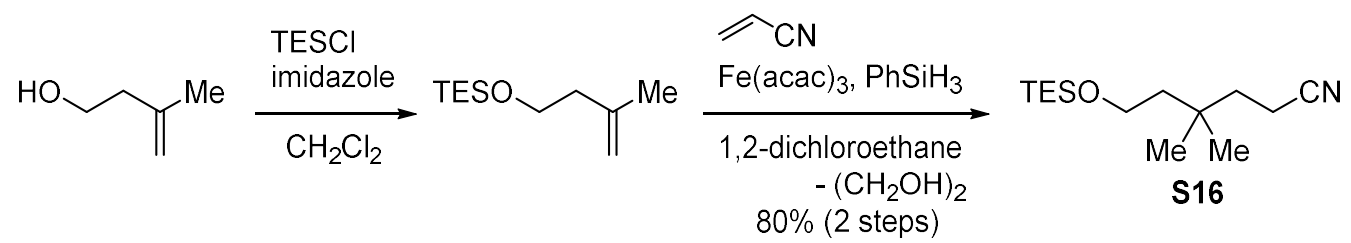

To a solution of imidazole (1.02 g, $15.0 \mathrm{mmol})$ and 3-methyl-3-butene-1-ol (1.01 mL, $10.0 \mathrm{mmol})$ in $\mathrm{CH}_{2} \mathrm{Cl}_{2}(20 \mathrm{~mL})$ was added TESCl $(2.01 \mathrm{~mL}, 12.0 \mathrm{mmol})$ at $0{ }^{\circ} \mathrm{C}$. The reaction mixture was then warmed up to room temperature and stirred for $1 \mathrm{~h}$. The reaction mixture was cooled down to $0{ }^{\circ} \mathrm{C}$ and added $\mathrm{MeOH}(1 \mathrm{~mL})$. After stirring at room temperature for several minutes, the solvent was removed under reduced pressure. The resulting mixture was filtered through a pad of Celite ${ }^{\circledR}$ and the residue was washed with hexane. The resulting filtrate was concentrated to give a crude oil.

To a crude solution in 1,2-dichloroethane and ethylene glycol $(100 \mathrm{~mL}, 5: 1)$ were added $\mathrm{Fe}(\mathrm{acac})_{3}$ (1.41 g, $4.00 \mathrm{mmol}$ ), acrylonitrile (1.97 mL, $30.0 \mathrm{mmol})$, and $\mathrm{PhSiH}_{3}(1.84 \mathrm{~mL}, 15.0 \mathrm{mmol})$ successively at room temperature. The reaction mixture was then warmed up to $60{ }^{\circ} \mathrm{C}$ and stirred for $1 \mathrm{~h}$. The reaction was cooled to room temperature and quenched with brine $(50 \mathrm{~mL})$. Two layers were separated, and the aqueous layer was extracted with $\mathrm{Et}_{2} \mathrm{O}(50 \mathrm{~mL} \times 3)$. The combined organic layer was washed with brine $(100 \mathrm{~mL})$, dried over $\mathrm{MgSO}_{4}$, filtered, and concentrated under reduced pressure. The resulting residue was purified by flash column chromatography $\left(\mathrm{SiO}_{2}\right.$, hexane:EtOAc $=20: 1)$ to afford $\mathbf{S 1 6}$ (2.04 g, $7.98 \mathrm{mmol}, 80 \%$ for 2 steps $)$ as a yellow oil ${ }^{[4]}$.

S16: ${ }^{1} \mathrm{H}$ NMR $\left(500 \mathrm{MHz}, \mathrm{CDCl}_{3}\right) \delta 3.66(\mathrm{t}, J=6.9 \mathrm{~Hz}, 2 \mathrm{H}), 2.31(\mathrm{t}, J=8.2 \mathrm{~Hz}, 2 \mathrm{H}), 1.67(\mathrm{t}, J=8.2 \mathrm{~Hz}$, $2 \mathrm{H}), 1.47(\mathrm{t}, J=6.9 \mathrm{~Hz}, 2 \mathrm{H}), 0.96(\mathrm{t}, J=7.8 \mathrm{~Hz}, 9 \mathrm{H}), 0.93(\mathrm{~s}, 6 \mathrm{H}), 0.60(\mathrm{q}, J=8.0 \mathrm{~Hz}, 6 \mathrm{H}) ;{ }^{13} \mathrm{C} \mathrm{NMR}$ $\left(126 \mathrm{MHz} \mathrm{CDCl}_{3}\right) \delta 120.5,59.1,43.1,37.6,32.2,26.9$ (2C), 12.4, 6.8 (3C), 4.3 (3C); FT-IR (ATR) v $2955,2875,2247,1459,1092,1001,726 \mathrm{~cm}^{-1}$; HRMS (FD) calcd for $\mathrm{C}_{14} \mathrm{H}_{30} \mathrm{NOSi}\left([\mathrm{M}+\mathrm{H}]^{+}\right): 256.2097$, found: 256.2085 . 


\section{4,4-Dimethyl-6-oxohexanenitrile (S11)}

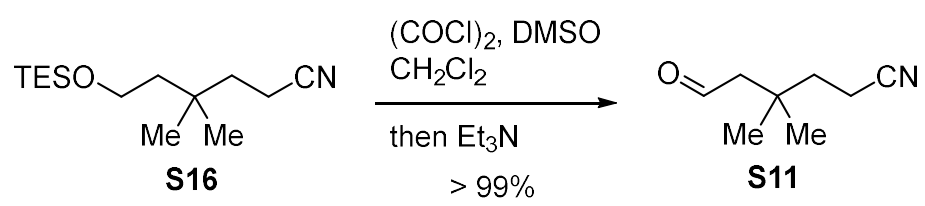

To a solution of oxalyl chloride $(822 \mu \mathrm{L}, 9.58 \mathrm{mmol})$ in $\mathrm{CH}_{2} \mathrm{Cl}_{2}(30 \mathrm{~mL})$ was added DMSO (1.36 $\mathrm{mL}, 19.2 \mathrm{mmol})$ in $\mathrm{CH}_{2} \mathrm{Cl}_{2}(5.0 \mathrm{~mL})$ at $-78{ }^{\circ} \mathrm{C}$, and the reaction mixture was stirred for $15 \mathrm{~min}$. To this mixture was added $\mathbf{S 1 6}(2.04 \mathrm{~g}, 7.98 \mathrm{mmol})$ in $\mathrm{CH}_{2} \mathrm{Cl}_{2}(3.0 \mathrm{~mL})$ and the reaction mixture was warmed up to $-40{ }^{\circ} \mathrm{C}$ over $30 \mathrm{~min}$. The resulting mixture was stirred for additional $20 \mathrm{~min}$ at the same temperature before $\mathrm{Et}_{3} \mathrm{~N}(3.28 \mathrm{~mL}, 23.5 \mathrm{mmol})$ was added. The reaction mixture was then warmed up to $0{ }^{\circ} \mathrm{C}$ and stirred for $20 \mathrm{~min}$. The reaction was quenched with saturated aqueous $\mathrm{NH}_{4} \mathrm{Cl}$ solution. Two layers were separated, and the aqueous layer was extracted with $\mathrm{Et}_{2} \mathrm{O}(20 \mathrm{~mL} \times 3)$. The combined organic layer was washed with brine $(50 \mathrm{~mL})$, dried over $\mathrm{MgSO}_{4}$, filtered, and concentrated under reduced pressure. The resulting residue was purified by flash column chromatography $\left(\mathrm{SiO}_{2}\right.$, hexane:EtOAc $=4: 1$ to 2:1) to afford $\mathbf{S 1 1}(1.11 \mathrm{~g}, 7.97 \mathrm{mmol},>99 \%)$ as a yellow oil.

S11: ${ }^{1} \mathrm{H}$ NMR $\left(500 \mathrm{MHz}, \mathrm{CDCl}_{3}\right) \delta 9.83(\mathrm{t}, J=2.9 \mathrm{~Hz}, 1 \mathrm{H}), 2.35-2.31(\mathrm{~m}, 4 \mathrm{H}), 1.78(\mathrm{t}, J=8.0 \mathrm{~Hz}$, 2H), $1.11(\mathrm{~s}, 6 \mathrm{H}) ;{ }^{13} \mathrm{C} \mathrm{NMR}\left(126 \mathrm{MHz}, \mathrm{CDCl}_{3}\right) \delta 201.8,119.8,53.9,37.3,33.1,26.7$ (2C), 12.3; FT-IR (ATR) $v 2964,2875,2738,2247,1716,1472 \mathrm{~cm}^{-1}$; HRMS (FI) calcd for $\mathrm{C}_{8} \mathrm{H}_{13} \mathrm{NO}\left(\mathrm{M}^{+}\right)$: 139.0997, found: 139.1004 .

\section{4,4-Dimethyl-2-((triisopropylsilyl)oxy)cyclopentane-1-carbonitrile (S12)}

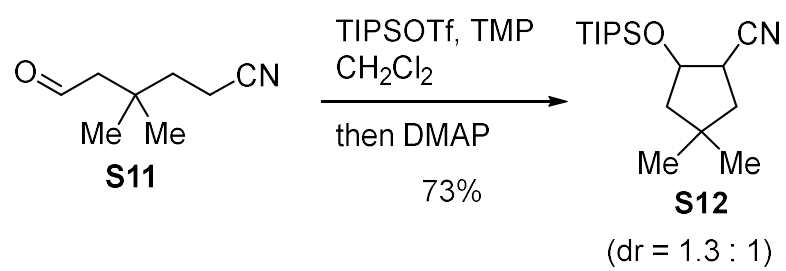

To a solution of $\mathbf{S 1 1}(1.12 \mathrm{~g}, 8.02 \mathrm{mmol})$ in $\mathrm{CH}_{2} \mathrm{Cl}_{2}(40.0 \mathrm{~mL})$ were added tetramethylpiperidine (TMP) $(2.05 \mathrm{~mL}, 12.0 \mathrm{mmol})$ and TIPSOTf $(3.23 \mathrm{~mL}, 12.0 \mathrm{mmol})$ successively at room temperature. After stirring for $45 \mathrm{~min}$, 4-dimethylaminopyridine (DMAP) $(95.1 \mathrm{mg}, 778 \mu \mathrm{mol})$ was added and the reaction mixture was stirred for additional $1 \mathrm{~h}$. The reaction was quenched with saturated aqueous $\mathrm{NH}_{4} \mathrm{Cl}$ solution. Two layers were separated, and the aqueous layer was extracted with $\mathrm{CH}_{2} \mathrm{Cl}_{2}$ (3 times). The combined organic layer was dried over $\mathrm{MgSO}_{4}$, filtered, and concentrated under reduced pressure. The resulting residue was purified by flash column chromatography $\left(\mathrm{SiO}_{2}\right.$, hexane:EtOAc $=50: 1$ to $20: 1)$ to afford $\mathbf{S 1 2}(\mathrm{dr}=1.3: 1,1.74 \mathrm{~g}, 5.89 \mathrm{mmol}, 73 \%)$ as a yellow oil. 
S12: ${ }^{1} \mathrm{H}$ NMR $\left(500 \mathrm{MHz}, \mathrm{CDCl}_{3}\right) \delta 4.61(\mathrm{q}, J=6.9 \mathrm{~Hz}, 0.37 \mathrm{H}), 4.52(\mathrm{q}, J=5.5 \mathrm{~Hz}, 0.63 \mathrm{H}), 2.97-$ $2.93(\mathrm{~m}, 0.63 \mathrm{H}), 2.85-2.80(\mathrm{~m}, 0.37 \mathrm{H}), 2.05-1.68(\mathrm{~m}, 4 \mathrm{H}), 1.20(\mathrm{~s}, 0.63 \times 3 \mathrm{H}), 1.11-1.05(\mathrm{~m}, 21 \mathrm{H}+$ $0.37 \times 6 \mathrm{H}), 0.98(\mathrm{~s}, 0.63 \times 3 \mathrm{H}) ;{ }^{13} \mathrm{C} \mathrm{NMR}\left(126 \mathrm{MHz}, \mathrm{CDCl}_{3}\right) \delta 122.4,120.3,77.8,74.1,50.1,49.9$, 43.3, 43.1, 38.1, 38.0, 36.9, 36.8, 30.9, 30.3, 30.1, 29.7, 18.0 (6C), 17.9 (6C), 12.1 (3C), 12.0 (3C); FT-IR (ATR) v 2944, 2867, 2240, 1463, 881, $681 \mathrm{~cm}^{-1}$; HRMS (FI) calcd for $\mathrm{C}_{17} \mathrm{H}_{33} \mathrm{NOSi}\left(\mathrm{M}^{+}\right)$: 295.2331, found: 295.2320 .

NOTE: DMAP was added to promote silylation of concomitant $\beta$-hydroxynitrile, but the reproducibility of this step was low.

\section{4,4-Dimethylcyclopentene-1-carbaldehyde (S14)}

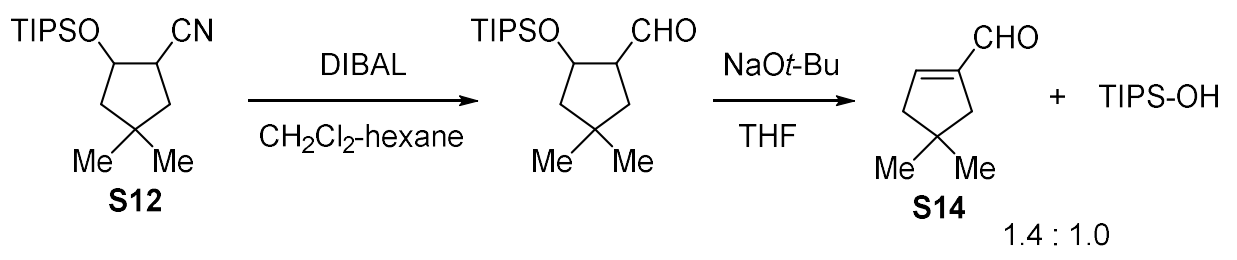

To a solution of $\mathbf{S 1 2}(887 \mathrm{mg}, 3.00 \mathrm{mmol})$ in $\mathrm{CH}_{2} \mathrm{Cl}_{2}(15 \mathrm{~mL})$ was added DIBAL $(1.03 \mathrm{~mol} / \mathrm{L}$ in hexane, $4.37 \mathrm{~mL}, 4.50 \mathrm{mmol}$ ) at $-78{ }^{\circ} \mathrm{C}$. The reaction mixture was then warmed up to $0{ }^{\circ} \mathrm{C}$ and stirred for $1.5 \mathrm{~h}$. The reaction was quenched with $10 \%$ aqueous $(+)$-tartaric acid solution $(10 \mathrm{~mL})$, and the biphasic mixture was stirred vigorously overnight. Two layers were separated, and the aqueous layer was extracted with $\mathrm{CH}_{2} \mathrm{Cl}_{2}(10 \mathrm{~mL} \times 3)$. The combined organic layer was dried over $\mathrm{MgSO}_{4}$, filtered, and concentrated under reduced pressure to afford a crude oil.

To a solution of the crude aldehyde in THF $(15 \mathrm{~mL})$ was added $\mathrm{NaOt}$-Bu $(577 \mathrm{mg}, 6.00 \mathrm{mmol})$ at $0{ }^{\circ} \mathrm{C}$. The reaction mixture was then warmed up to room temperature and stirred for $40 \mathrm{~min}$. The reaction was quenched with saturated aqueous $\mathrm{NH}_{4} \mathrm{Cl}$ solution $(15 \mathrm{~mL})$. Two layers were separated, and the aqueous layer was extracted with $\mathrm{Et}_{2} \mathrm{O}(15 \mathrm{~mL} \times 3)$. The combined organic layer was dried over $\mathrm{MgSO}_{4}$, filtered, and concentrated under reduced pressure. The resulting residue was purified roughly by flash column chromatography $\left(\mathrm{SiO}_{2}\right.$, pentane:Et $\mathrm{E}_{2} \mathrm{O}=30: 1,20: 1$ to 5:1) to afford $\mathbf{S 1 4}$ along with triisopropylsilanol (TIPSOH) $(480 \mathrm{mg}, \mathbf{S 1 4}: \mathrm{TIPSOH}=1.4: 1)$. The mixture of products was directly used for the next step without further purification. 


\section{Preparation of other $\alpha, \beta$-unsaturated aldehydes}

\section{2-Methyleneoctanal}

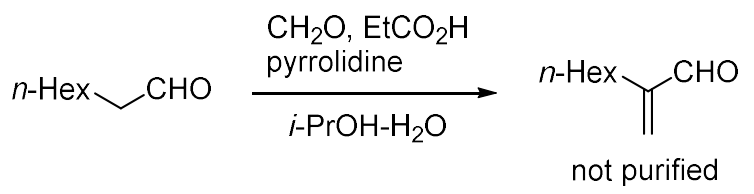

To a solution of $n$-octanal $(469 \mu \mathrm{L}, 3.00 \mathrm{mmol})$ in $i$-PrOH $(300 \mu \mathrm{L})$ were added $37 \%$ aqueous $\mathrm{CH}_{2} \mathrm{O}$ solution $(221 \mu \mathrm{L}, 3.00 \mathrm{mmol})$, propionic acid $(22.2 \mu \mathrm{L}, 300 \mu \mathrm{mol})$, and pyrrolidine $(24.8 \mu \mathrm{L}, 300$ $\mu \mathrm{mol})$ successively. The reaction mixture was then warmed up to $45{ }^{\circ} \mathrm{C}$ and stirred for $4 \mathrm{~h}$. The reaction was quenched with saturated aqueous $\mathrm{NaHCO}_{3}$ solution $(1.0 \mathrm{~mL})$. Two layers were separated, and the aqueous layer was extracted with $\mathrm{CH}_{2} \mathrm{Cl}_{2}(1.0 \mathrm{~mL} \times 5)$. The combined organic layer was dried over $\mathrm{MgSO}_{4}$, filtered, and concentrated under reduced pressure to afford the title compound as a crude product. The resulting crude product was directly used for next steps without further purification ${ }^{[5]}$.

\section{2-Methylene-5-((triisopropylsilyl)oxy)pentanal (S18)}

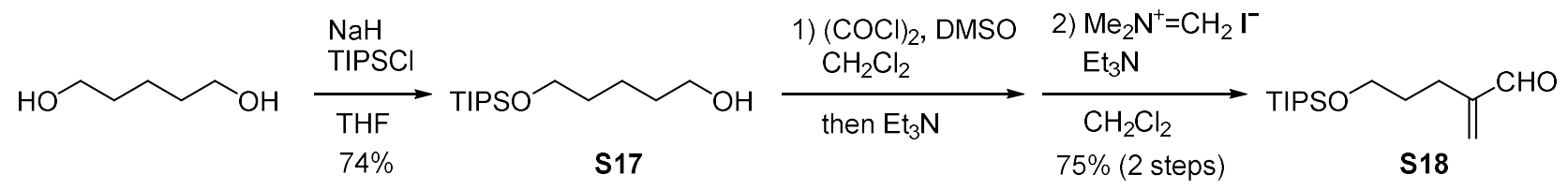

S17

$\mathrm{NaH}$ (55\% dispersion in mineral oil, $873 \mathrm{mg}, 20.0 \mathrm{mmol})$ was washed with hexane and dissolved with THF $(40 \mathrm{~mL})$. To this mixture was added 1,5-pentanediol $(2.10 \mathrm{~mL}, 20.0 \mathrm{mmol})$ at $0{ }^{\circ} \mathrm{C}$ and the reaction mixture was then warmed up to room temperature. After stirring for $1 \mathrm{~h}$, the reaction mixture was cooled down to $0{ }^{\circ} \mathrm{C}$ and TIPSCl $(4.28 \mathrm{~mL}, 20.0 \mathrm{mmol})$ was added. The reaction mixture was then warmed up to room temperature and stirred for $3 \mathrm{~h}$. The reaction was quenched with $\mathrm{H}_{2} \mathrm{O}(20 \mathrm{~mL})$. Two layers were separated, and the aqueous layer was extracted with $\mathrm{Et}_{2} \mathrm{O}(20$ $\mathrm{mL} \times 3)$. The combined organic layer was dried over $\mathrm{MgSO}_{4}$, filtered, and concentrated under reduced pressure. The resulting residue was purified by flash column chromatography $\left(\mathrm{SiO}_{2}\right.$, hexane:EtOAc $=5: 1)$ to afford $\mathbf{S 1 7}(3.83 \mathrm{~g}, 14.7 \mathrm{mmol}, 74 \%$ yield $)$ as a colorless oil.

S17: ${ }^{1} \mathrm{H}$ spectral property was identical to that reported ${ }^{[6]}$. 


\section{S18}

To a solution of oxalyl chloride $(515 \mu \mathrm{L}, 6.00 \mathrm{mmol})$ in $\mathrm{CH}_{2} \mathrm{Cl}_{2}(21 \mathrm{~mL})$ was added DMSO (852 $\mu \mathrm{L}, 12.0 \mathrm{mmol})$ in $\mathrm{CH}_{2} \mathrm{Cl}_{2}(2.0 \mathrm{~mL})$ at $-78^{\circ} \mathrm{C}$. After stirring for $25 \mathrm{~min}, \mathbf{S 1 7}(1.30 \mathrm{~g}, 5.00 \mathrm{mmol})$ in $\mathrm{CH}_{2} \mathrm{Cl}_{2}(2.0 \mathrm{~mL})$ was added, and the reaction mixture was stirred for additional $15 \mathrm{~min}$ at the same temperature before $\mathrm{Et}_{3} \mathrm{~N}(3.48 \mathrm{~mL}, 25.0 \mathrm{mmol})$ was added. The reaction mixture was then warmed up to room temperature and stirred for $15 \mathrm{~min}$. The reaction was quenched with saturated aqueous $\mathrm{NaHCO}_{3}$ solution $(20 \mathrm{~mL})$. Two layers were separated, and the aqueous layer was extracted with $\mathrm{CH}_{2} \mathrm{Cl}_{2}(20 \mathrm{~mL} \times 3)$. The combined organic layer was dried over $\mathrm{MgSO}_{4}$, filtered, and concentrated under reduced pressure to afford a crude oil.

To a solution of the crude aldehyde in $\mathrm{CH}_{2} \mathrm{Cl}_{2}(25 \mathrm{~mL})$ were added $N, N$-dimethylmethylene ammonium iodide (Eshenmoser's salt) (1.85 g, $10.0 \mathrm{mmol})$ and $\mathrm{Et}_{3} \mathrm{~N}$ (1.95 mL, $\left.14.0 \mathrm{mmol}\right)$ successively at $0{ }^{\circ} \mathrm{C}$. The reaction mixture was then warmed up to room temperature and stirred for $15 \mathrm{~min}$. The reaction was quenched with saturated aqueous $\mathrm{NaHCO}_{3}$ solution $(20 \mathrm{~mL})$. Two layers were separated, and the aqueous layer was extracted with $\mathrm{CH}_{2} \mathrm{Cl}_{2}(20 \mathrm{~mL} \times 3)$. The combined organic layer was dried over $\mathrm{MgSO}_{4}$, filtered, and concentrated under reduced pressure. The resulting residue was purified by flash column chromatography $\left(\mathrm{SiO}_{2}\right.$, hexane:EtOAc $\left.=20: 1\right)$ to afford $\mathbf{S 1 8}$ (1.02 g, $3.76 \mathrm{mmol}, 75 \%$ for 2 steps) as a colorless oil.

S18: ${ }^{1} \mathrm{H}$ NMR $\left(500 \mathrm{MHz}, \mathrm{CDCl}_{3}\right) \delta 9.55(\mathrm{~s}, 1 \mathrm{H}), 6.28(\mathrm{~s}, 1 \mathrm{H}), 6.01(\mathrm{~s}, 1 \mathrm{H}), 3.70(\mathrm{t}, J=6.3 \mathrm{~Hz}, 2 \mathrm{H})$, $2.34(\mathrm{t}, J=7.4 \mathrm{~Hz}, 2 \mathrm{H}), 1.72-1.67(\mathrm{~m}, 2 \mathrm{H}), 1.08-1.05(\mathrm{~m}, 21 \mathrm{H}) ;{ }^{13} \mathrm{C} \mathrm{NMR}\left(126 \mathrm{MHz}, \mathrm{CDCl}_{3}\right) \delta$ 194.7, 150.0, 134.1, 62.5, 30.9, 24.3, 18.0 (6C), 12.0 (3C); FT-IR (ATR) v 2942, 2865, 2341, 1697 , 1463, 1105, 881, $679 \mathrm{~cm}^{-1}$; HRMS (FI) calcd for $\mathrm{C}_{15} \mathrm{H}_{30} \mathrm{O}_{2} \mathrm{Si}\left(\mathrm{M}^{+}\right)$: 270.2015, found: 270.2005 .

\section{1-Cyclopentenecarbaldehyde}

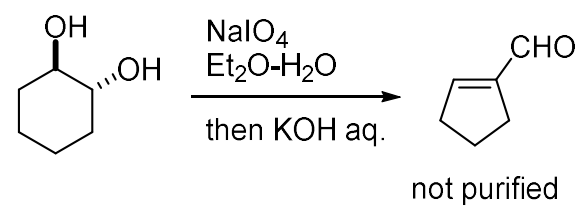

To a suspension of $\mathrm{NaIO}_{4}(512 \mathrm{mg}, 2.39 \mathrm{mmol})$ in $\mathrm{Et}_{2} \mathrm{O}(3.0 \mathrm{~mL})$ was added trans-1,2cyclohexanediol $(233 \mathrm{mg}, 2.00 \mathrm{mmol})$ in $\mathrm{H}_{2} \mathrm{O}(5.0 \mathrm{~mL})$ at room temperature. After vigorous stirring for $30 \mathrm{~min}, 20 \%$ aqueous $\mathrm{KOH}$ solution $(550 \mu \mathrm{L})$ was added, and the reaction mixture was stirred for $1 \mathrm{~h}$ at the same temperature. The two layers were separated, and the aqueous layer was extracted with $\mathrm{Et}_{2} \mathrm{O}(8.0 \mathrm{~mL} \times 3)$. The combined organic layer was dried over $\mathrm{MgSO}_{4}$, filtered, and concentrated under reduced pressure to afford the title compound $(133 \mathrm{mg}, 1.38 \mathrm{mmol}, 69 \%$ crude yield). The resulting crude product was directly used for next steps without further purification. 


\section{Procedure A: Aldol reaction}

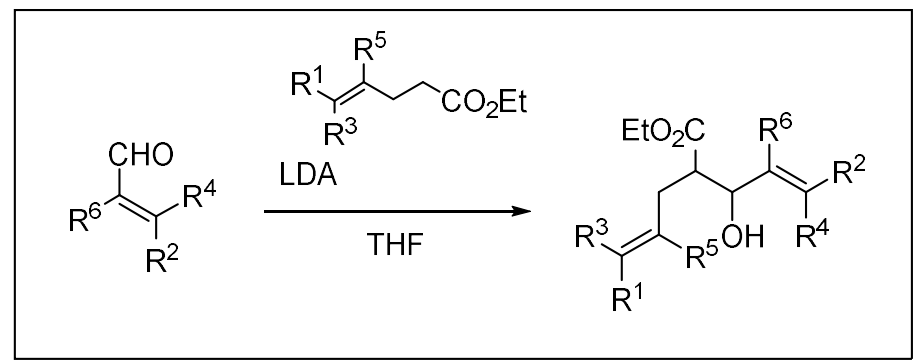

To a solution of $i-\mathrm{Pr}_{2} \mathrm{NH}$ in THF $(0.24 \mathrm{~mol} / \mathrm{L}, 1.2$ equiv.) was added $n$-BuLi $(2.69 \mathrm{~mol} / \mathrm{L}$ in hexane, 1.2 equiv.) at $0{ }^{\circ} \mathrm{C}$ and stirred for $30 \mathrm{~min}$. The reaction mixture was then cooled down to $-78^{\circ} \mathrm{C}$ and ester (1.2 equiv.) in THF was added. After stirring for $30 \mathrm{~min}$, aldehyde in THF was added and the resulting mixture was stirred at appropriate temperature $\left(-78--30{ }^{\circ} \mathrm{C}\right)$ until no further changes in TLC spots were observed (10-30 min). The reaction was quenched with saturated aqueous $\mathrm{NH}_{4} \mathrm{Cl}$ solution. Two layers were separated, and the aqueous layer was extracted with EtOAc (3 times). The combined organic layer was dried over $\mathrm{MgSO}_{4}$, filtered, and concentrated under reduced pressure. The resulting residue was purified by flash column chromatography $\left(\mathrm{SiO}_{2}\right.$, hexane:EtOAc $=10: 1$ to $3: 1)$ to afford the product.

For small-scale reactions ( $<0.5 \mathrm{mmol})$, LDA(ca. $1.0 \mathrm{~mol} / \mathrm{L}$ in THF-hexane) was prepared in another flask from $i-\mathrm{Pr}_{2} \mathrm{NH}(281 \mu \mathrm{L}, 2.00 \mathrm{mmol})$ and $n$-BuLi $(2.69 \mathrm{~mol} / \mathrm{L}$ in hexane, $743 \mu \mathrm{L}, 2.00 \mathrm{mmol})$ in THF $(1.26 \mathrm{~mL})$ at $0{ }^{\circ} \mathrm{C}$. The LDA solution (1.2 equiv.) was added via a syringe to a solution of ester in $\operatorname{THF}(0.24 \mathrm{~mol} / \mathrm{L}, 1.2$ equiv. $)$ at $-78^{\circ} \mathrm{C}$.

\section{ß-Hydroxy ester 3a}

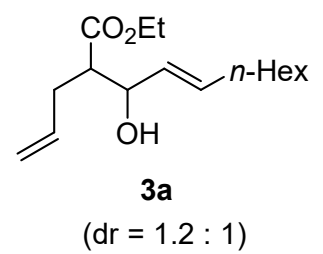

According to procedure A (reaction conditions: $\left.-78^{\circ} \mathrm{C}, 10 \mathrm{~min}\right), i-\operatorname{Pr}_{2} \mathrm{NH}(843$ $\mu \mathrm{L}, 6.00 \mathrm{mmol}), n$-BuLi (2.69 mol/L in hexane, $2.23 \mathrm{~mL}, 6.00 \mathrm{mmol})$, ethyl 4pentenoate $(854 \mu \mathrm{L}, 6.00 \mathrm{mmol})$, and trans-2-nonenal $(825 \mu \mathrm{L}, 5.00 \mathrm{mmol})$ gave 3a $(\mathrm{dr}=1.2: 1,1.33 \mathrm{~g}, 4.96 \mathrm{mmol}, 99 \%$ yield $)$ as a yellow oil.

3a: ${ }^{1} \mathrm{H}$ NMR $\left(500 \mathrm{MHz}, \mathrm{CDCl}_{3}\right) \delta 5.83-5.69(\mathrm{~m}, 2 \mathrm{H}), 5.49-5.41(\mathrm{~m}, 1 \mathrm{H}), 5.09-5.01(\mathrm{~m}, 2 \mathrm{H}), 4.27$ $(\mathrm{t}, J=6.3 \mathrm{~Hz}, 0.55 \mathrm{H}), 4.21-4.11(\mathrm{~m}, 2 \mathrm{H}+0.45 \mathrm{H}), 2.61(\mathrm{dt}, J=10.3,4.6 \mathrm{~Hz}, 0.55 \mathrm{H}), 2.53(\mathrm{q}, J=$ $7.1 \mathrm{~Hz}, 0.45 \mathrm{H}), 2.47-2.34(\mathrm{~m}, 2 \mathrm{H}), 2.06-2.01(\mathrm{~m}, 2 \mathrm{H}), 1.36-1.24(\mathrm{~m}, 11 \mathrm{H}), 0.88(\mathrm{t}, J=6.9 \mathrm{~Hz}, 3 \mathrm{H})$; ${ }^{13} \mathrm{C}$ NMR $\left(126 \mathrm{MHz}, \mathrm{CDCl}_{3}\right) \delta 174.4,173.8,135.5,134.7,134.2,134.1,129.9,128.9,117.1,116.7$, $73.1,72.9,60.5,60.5,51.1,51.1,33.5,32.2,32.0,32.0,31.6,29.0,29.0,28.8,22.6,14.2$ (overlapped), 14.0 (overlapped) (3 peaks missing); FT-IR (ATR) v 3449 (br), 3079, 2957, 2925, 2855, 1732, 1715, $1178 \mathrm{~cm}^{-1}$; HRMS (FI) calcd for $\mathrm{C}_{16} \mathrm{H}_{28} \mathrm{O}_{3}\left(\mathrm{M}^{+}\right)$: 268.2038, found: 268.2045 . 


\section{ß-Hydroxy ester 3b}

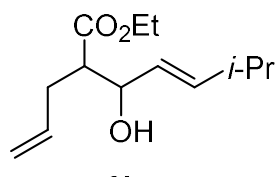

$3 b$

$(\mathrm{dr}=1.3: 1)$

According to procedure A (reaction conditions: $\left.-78^{\circ} \mathrm{C}, 15 \mathrm{~min}\right), i-\mathrm{Pr}_{2} \mathrm{NH}(843$ $\mu \mathrm{L}, 6.00 \mathrm{mmol}), n$-BuLi (2.69 mol/L in hexane, $2.23 \mathrm{~mL}, 6.00 \mathrm{mmol})$, ethyl 4pentenoate $(854 \mu \mathrm{L}, 6.00 \mathrm{mmol})$, and trans-4-methyl-2-pentenal $(581 \mu \mathrm{L}, 5.00$ mmol) gave 3b $(\mathrm{dr}=1.3: 1,1.10 \mathrm{~g}, 4.86 \mathrm{mmol}, 97 \%$ yield $)$ as a yellow oil.

3b: ${ }^{1} \mathrm{H}$ NMR $\left(500 \mathrm{MHz}, \mathrm{CDCl}_{3}\right) \delta 5.83-5.66(\mathrm{~m}, 2 \mathrm{H}), 5.45-5.36(\mathrm{~m}, 1 \mathrm{H}), 5.09-5.01(\mathrm{~m}, 2 \mathrm{H}), 4.27$ $(\mathrm{t}, J=6.3 \mathrm{~Hz}, 0.57 \mathrm{H}), 4.21-4.11(\mathrm{~m}, 2 \mathrm{H}+0.43 \mathrm{H}), 2.63-2.59(\mathrm{~m}, 0.57 \mathrm{H}), 2.54(\mathrm{q}, J=6.9 \mathrm{~Hz}, 0.43 \mathrm{H})$, 2.47-2.26 (m, 3H), 1.28-1.24 (m, 3H), 1.00-0.98 (m, 6H); $\left.{ }^{13} \mathrm{C} \mathrm{NMR} \mathrm{(126} \mathrm{MHz,} \mathrm{CDCl}_{3}\right) \delta 174.4$, 173.8, 140.8 (overlapped), 135.5, 134.7, 127.0, 126.0, 117.1, 116.7, 73.1, 73.0, 60.5, 60.5, 51.2, 51.1, 33.5, 32.0, 30.7, 22.2 (2C), 22.2 (2C), 14.3, 14.2 (1 peak missing); FT-IR (ATR) v 3448 (br), 3079, 2959, 2870, 1732, 1715, 1178, $971 \mathrm{~cm}^{-1}$; HRMS (FI) calcd for $\mathrm{C}_{13} \mathrm{H}_{22} \mathrm{O}_{3}\left(\mathrm{M}^{+}\right): 226.1569$, found: 226.1573.

\section{$\beta$-Hydroxy ester S19a}

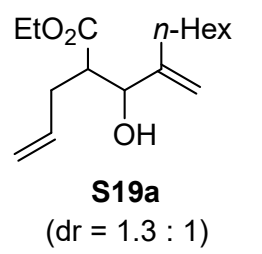

According to procedure A (reaction conditions: $-78^{\circ} \mathrm{C}, 25 \mathrm{~min}$ ), $i$ - $\operatorname{Pr}_{2} \mathrm{NH}(169 \mu \mathrm{L}$, $1.20 \mathrm{mmol}), n$-BuLi (2.69 mol/L in hexane, $446 \mu \mathrm{L}, 1.20 \mathrm{mmol})$, ethyl 4-pentenoate $(171 \mu \mathrm{L}, 1.20 \mathrm{mmol}$ ), and crude 2-methyleneoctanal (prepared from $1.00 \mathrm{mmol}$ of $n$-octanal) gave S19a $(\mathrm{dr}=1.3: 1,192 \mathrm{mg}, 715 \mu \mathrm{mol}, 72 \%$ yield for 2 steps from $n$-octanal) as a colorless oil.

S19a: ${ }^{1} \mathrm{H}$ NMR (500 MHz, $\left.\mathrm{CDCl}_{3}\right) \delta 5.84-5.71(\mathrm{~m}, 1 \mathrm{H}), 5.14-4.93(\mathrm{~m}, 4 \mathrm{H}), 4.35(\mathrm{~d}, J=5.2 \mathrm{~Hz}$, $0.43 \mathrm{H}), 4.19-4.11(\mathrm{~m}, 2 \mathrm{H}+0.57 \mathrm{H}), 2.74-2.65(\mathrm{~m}, 1 \mathrm{H}), 2.42-2.30(\mathrm{~m}, 2 \mathrm{H}), 2.14-1.92(\mathrm{~m}, 2 \mathrm{H}), 1.50-$ $1.44(\mathrm{~m}, 2 \mathrm{H}), 1.32-1.23(\mathrm{~m}, 9 \mathrm{H}), 0.89(\mathrm{t}, J=6.9 \mathrm{~Hz}, 3 \mathrm{H}) ;{ }^{13} \mathrm{C} \mathrm{NMR}\left(126 \mathrm{MHz}, \mathrm{CDCl}_{3}\right) \delta 174.6,174.5$, 149.2, 148.3, 135.8, 134.6, 117.3, 116.6, 111.3, 111.2, 75.4, 74.6, 60.6 (overlapped), 48.7, 48.6, 34.0, 31.9, 31.7, 31.3, 31.0, 29.2 , 29.1, 27.8, 27.7, 22.6, 14.3, 14.2, 14.1 (overlapped) (2 peaks missing); FTIR (ATR) v 3471 (br), 3080, 2956, 2927, 2858, 1733, 1715, 1177, 1030, $909 \mathrm{~cm}^{-1}$; HRMS (FI) calcd for $\mathrm{C}_{16} \mathrm{H}_{28} \mathrm{O}_{3}\left(\mathrm{M}^{+}\right)$: 268.2038, found: 268.2035. 


\section{$\beta$-Hydroxy ester S19b}

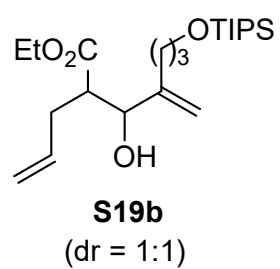

According to procedure $\mathrm{A}$ (reaction conditions: $-78^{\circ} \mathrm{C}, 20 \mathrm{~min} ;-30^{\circ} \mathrm{C}, 10 \mathrm{~min}$ ), LDA (ca. $1.0 \mathrm{~mol} / \mathrm{L}$ in THF-hexane, $543 \mu \mathrm{L}, 543 \mu \mathrm{mol}$ ), ethyl 4-pentenoate (77.3 $\mu \mathrm{L}, 543 \mu \mathrm{mol})$, and $\mathbf{S 1 8}(123 \mathrm{mg}, 453 \mu \mathrm{mol})$ gave $\mathbf{S 1 9 b}(\mathrm{dr}=1: 1,126 \mathrm{mg}, 315$ $\mu \mathrm{mol}, 70 \%$ yield) as a colorless oil.

S19b: ${ }^{1} \mathrm{H}$ NMR $\left(500 \mathrm{MHz}, \mathrm{CDCl}_{3}\right) \delta 5.83-5.69(\mathrm{~m}, 1 \mathrm{H}), 5.15-4.96(\mathrm{~m}, 4 \mathrm{H}), 4.35(\mathrm{~d}, J=5.2 \mathrm{~Hz}$, $0.5 \mathrm{H}), 4.21-4.11(\mathrm{~m}, 2 \mathrm{H}+0.5 \mathrm{H}), 3.74-3.70(\mathrm{~m}, 2 \mathrm{H}), 2.75-2.55(\mathrm{~m}, 1 \mathrm{H}), 2.43-2.29(\mathrm{~m}, 2 \mathrm{H}), 2.23-$ $2.04(\mathrm{~m}, 2 \mathrm{H}), 1.79-1.68(\mathrm{~m}, 2 \mathrm{H}), 1.28-1.23(\mathrm{~m}, 3 \mathrm{H}), 1.11-1.05(\mathrm{~m}, 21 \mathrm{H}) ;{ }^{13} \mathrm{C} \mathrm{NMR}(126 \mathrm{MHz}$, $\left.\mathrm{CDCl}_{3}\right) \delta 174.6,174.4,148.8,147.9,135.8,134.6,117.3,116.5,111.8,111.6,75.6,74.9,62.8,62.8$, $60.6,60.5,48.9,48.7,34.0,31.4,31.3,31.2,28.0,27.6,18.0(6 \mathrm{C}+6 \mathrm{C}), 14.3,14.2,11.9(3 \mathrm{C}+3 \mathrm{C})$; FT-IR (ATR) v 3491 (br), 3080, 2942, 2866, 1734, 1716, $1103 \mathrm{~cm}^{-1}$; HRMS (FD) calcd for $\mathrm{C}_{22} \mathrm{H}_{43} \mathrm{O}_{4} \mathrm{Si}\left([\mathrm{M}+\mathrm{H}]^{+}\right)$: 399.2931, found: 399.2924 .

\section{$\beta$-Hydroxy ester S19c}

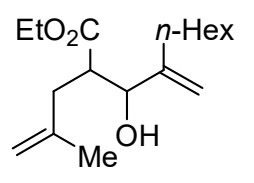

S19c $(\mathrm{dr}=1.4: 1)$

According to procedure A (reaction conditions: $\left.-78{ }^{\circ} \mathrm{C}, 15 \mathrm{~min}\right), i-\mathrm{Pr}_{2} \mathrm{NH}(271 \mu \mathrm{L}$, $1.93 \mathrm{mmol}), n$-BuLi (2.69 mol/L in hexane, $717 \mu \mathrm{L}, 1.93 \mathrm{mmol})$, ethyl 4-methyl-4pentenoate $(229 \mathrm{mg}, 1.61 \mathrm{mmol})$, and crude 2-methyleneoctanal $(271 \mathrm{mg}$, ca. 1.93 mmol) gave $\mathbf{S 1 9 c}(\mathrm{dr}=1.4: 1,269 \mathrm{mg}, 952 \mu \mathrm{mol}, 59 \%$ yield $)$ as a yellow oil.

S19c: ${ }^{1} \mathrm{H}$ NMR $\left(500 \mathrm{MHz}, \mathrm{CDCl}_{3}\right) \delta 5.14(\mathrm{~s}, 0.42 \mathrm{H}), 5.05(\mathrm{~s}, 0.58 \mathrm{H}), 4.96(\mathrm{~s}, 0.42 \mathrm{H}), 4.93(\mathrm{~s}, 0.58 \mathrm{H})$, $4.77-4.71(\mathrm{~m}, 2 \mathrm{H}), 4.33(\mathrm{~d}, J=5.2 \mathrm{~Hz}, 0.42 \mathrm{H}), 4.16-4.08(\mathrm{~m}, 2 \mathrm{H}+0.58 \mathrm{H}), 2.88-2.78(\mathrm{~m}, 1 \mathrm{H})$, 2.44-2.39 (m, 1H), 2.31 (dd, $J=15.1,3.2 \mathrm{~Hz}, 0.42 \mathrm{H}), 2.22(\mathrm{dd}, J=14.3,5.2 \mathrm{~Hz}, 0.58 \mathrm{H}), 2.15-1.92$ (m, 2H), 1.77-1.72 (m, 3H), 1.51-1.41 (m, 2H), 1.35-1.22 (m, 9H), $0.89(\mathrm{t}, J=6.6 \mathrm{~Hz}, 3 \mathrm{H}) ;{ }^{13} \mathrm{C}$ $\mathrm{NMR}\left(126 \mathrm{MHz}, \mathrm{CDCl}_{3}\right) \delta 174.7$ (overlapped), 149.4, 148.2, 143.2, 142.2, 112.6, 111.8, 111.3, 111.2, 75.8, 74.8, 60.6, 60.5 , 47.4, 47.2, 38.1, 34.8, 31.8, 31.7, 31.3, 29.1, 27.8, 22.6, 22.3, 22.1, 14.2, 14.2, 14.1 (overlapped) (4 peaks missing); FT-IR (ATR) v 3478 (br), 3078, 2956, 2927, 2857, 1734, 1716, 1161, 1028, $896 \mathrm{~cm}^{-1}$; HRMS (FD) calcd for $\mathrm{C}_{17} \mathrm{H}_{30} \mathrm{O}_{3}\left(\mathrm{M}^{+}\right)$: 282.2195, found: 282.2207 . 


\section{$\beta$-Hydroxy ester S19d}

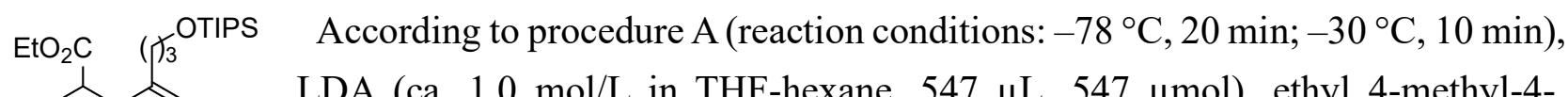
LDA (ca. $1.0 \mathrm{~mol} / \mathrm{L}$ in THF-hexane, $547 \mu \mathrm{L}, 547 \mu \mathrm{mol})$, ethyl 4-methyl-4pentenoate $(77.8 \mathrm{mg}, 547 \mu \mathrm{mol})$, and $\mathbf{S 1 8}(123 \mathrm{mg}, 455 \mu \mathrm{mol})$ gave $\mathbf{S 1 9 d}(\mathrm{dr}=$ 1:1, $119 \mathrm{mg}, 287 \mu \mathrm{mol}, 63 \%$ yield) as a yellow oil.

$(\mathrm{dr}=1: 1)$

S19d: ${ }^{1} \mathrm{H}$ NMR $\left(500 \mathrm{MHz}, \mathrm{CDCl}_{3}\right) \delta 5.15(\mathrm{~s}, 0.5 \mathrm{H}), 5.08(\mathrm{~s}, 0.5 \mathrm{H}), 4.97(\mathrm{~d}, J=8.6 \mathrm{~Hz}, 1 \mathrm{H}), 4.77-$ $4.70(\mathrm{~m}, 2 \mathrm{H}), 4.32(\mathrm{~d}, J=5.7 \mathrm{~Hz}, 0.5 \mathrm{H}), 4.19-4.07(\mathrm{~m}, 2 \mathrm{H}+0.5 \mathrm{H}), 3.74-3.70(\mathrm{~m}, 2 \mathrm{H}), 2.89-2.79$ $(\mathrm{m}, 1 \mathrm{H}), 2.44-2.33(\mathrm{~m}, 1 \mathrm{H}+0.5 \mathrm{H}), 2.23-2.04(\mathrm{~m}, 2 \mathrm{H}+0.5 \mathrm{H}), 1.77-1.70(\mathrm{~m}, 5 \mathrm{H}), 1.26-1.21(\mathrm{~m}$, $3 \mathrm{H}), 1.11-1.05(\mathrm{~m}, 21 \mathrm{H}) ;{ }^{13} \mathrm{C} \mathrm{NMR}\left(126 \mathrm{MHz}, \mathrm{CDCl}_{3}\right) \delta 174.7,174.7,149.0,147.8,143.3,142.2$, 112.6, 111.9, 111.8, 111.5, 75.9, 75.2, 62.9, 62.8, 60.5, 60.5, 47.7, 47.2, 38.1, 35.0, 31.4, 31.3, 27.9, 27.7, 22.3, 22.1, $18.0(6 \mathrm{C}+6 \mathrm{C}), 14.2,14.2,11.9(3 \mathrm{C}+3 \mathrm{C})$; FT-IR (ATR) v 3482 (br), 3078, 3942, 2866, 1735, 1716, 1103, $882 \mathrm{~cm}^{-1}$; HRMS (FD) calcd for $\mathrm{C}_{23} \mathrm{H}_{45} \mathrm{O}_{4} \mathrm{Si}\left([\mathrm{M}+\mathrm{H}]^{+}\right)$: 413.3087, found: 413.3088 .

\section{ß-Hydroxy ester S15a}

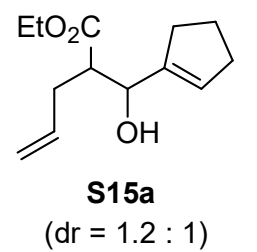

According to procedure A (reaction conditions: $\left.-78{ }^{\circ} \mathrm{C}, 15 \mathrm{~min}\right), i-\mathrm{Pr}_{2} \mathrm{NH}(233 \mu \mathrm{L}$, $1.66 \mathrm{mmol}), n$-BuLi $(2.69 \mathrm{~mol} / \mathrm{L}$ in hexane, $617 \mu \mathrm{L}, 1.66 \mathrm{mmol})$, ethyl 4-pentenoate $(236 \mu \mathrm{L}, 1.66 \mathrm{mmol})$, and crude cyclopentene-1-carboxaldehyde (133 mg, ca. 1.38 mmol) gave $\mathbf{S 1 5 a}(\mathrm{dr}=1.2: 1,215 \mathrm{mg}, 959 \mu \mathrm{mol}, 48 \%$ yield for 2 steps from trans1,2-cyclohexanediol) as a yellow oil.

S15a: ${ }^{1} \mathrm{H}$ NMR (500 MHz, $\left.\mathrm{CDCl}_{3}\right) \delta$ 5.84-5.66 (m, 2H), 5.10-5.00 (m, 2H), $4.52(\mathrm{~d}, J=5.2 \mathrm{~Hz}$, $0.45 \mathrm{H}), 4.38(\mathrm{~d}, J=6.9 \mathrm{~Hz}, 0.55 \mathrm{H}), 4.18-4.12(\mathrm{~m}, 2 \mathrm{H}), 2.71-2.65(\mathrm{~m}, 1 \mathrm{H}), 2.47-2.20(\mathrm{~m}, 6 \mathrm{H})$, 1.93-1.86 (m, 2H), 1.26-1.23 (m, 3H); $\left.{ }^{13} \mathrm{C} \mathrm{NMR} \mathrm{(126} \mathrm{MHz,} \mathrm{CDCl}_{3}\right) \delta 174.7,174.3,144.5,143.7$, 135.8, 134.7, 127.6, 127.3, 117.1, 116.5, 71.6, 71.5, 60.5 (overlapped), 49.4, 49.1, 33.8, 32.2, 32.1, 31.5, 31.4, 31.1, 23.3, 23.3, 14.2, 14.2; FT-IR (ATR) v 3462 (br), 3078, 2951, 2847, 1731, 1715, 1176, $1029 \mathrm{~cm}^{-1}$; HRMS (FI) calcd for $\mathrm{C}_{13} \mathrm{H}_{20} \mathrm{O}_{3}\left(\mathrm{M}^{+}\right)$: 224.1412, found: 224.1416. 


\section{$\beta$-Hydroxy ester S15b}

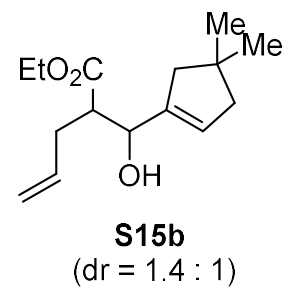

According to procedure A (reaction conditions: $\left.-78{ }^{\circ} \mathrm{C}, 15 \mathrm{~min}\right), i$ - $\operatorname{Pr}_{2} \mathrm{NH}(843$ $\mu \mathrm{L}, 6.00 \mathrm{mmol}), n$-BuLi (2.69 mol/L in hexane, $2.23 \mathrm{~mL}, 6.00 \mathrm{mmol})$, ethyl 4pentenoate $(854 \mu \mathrm{L}, 6.00 \mathrm{mmol}$ ), and $\mathbf{S 1 4}$ (mixture with TIPSOH, S14 : TIPSOH $=1.4: 1,480 \mathrm{mg})$ gave $\mathbf{S 1 5 b}(\mathrm{dr}=1.4: 1,394 \mathrm{mg}, 1.56 \mathrm{mmol}, 52 \%$ yield for 3 steps from $\mathbf{S 1 2}$ ) as a yellow oil.

S15b: ${ }^{1} \mathrm{H}$ NMR (500 MHz, $\left.\mathrm{CDCl}_{3}\right) \delta$ 5.85-5.71 (m, 1H), $5.57(\mathrm{~s}, 0.42 \mathrm{H}), 5.54(\mathrm{~s}, 0.58 \mathrm{H}), 5.11-5.00$ $(\mathrm{m}, 2 \mathrm{H}), 4.46(\mathrm{~d}, J=5.2 \mathrm{~Hz}, 0.42 \mathrm{H}), 4.30(\mathrm{~d}, J=6.3 \mathrm{~Hz}, 0.58 \mathrm{H}), 4.18-4.11(\mathrm{~m}, 2 \mathrm{H}), 2.68-2.61(\mathrm{~m}$, $1 \mathrm{H}), 2.47-2.30(\mathrm{~m}, 2 \mathrm{H}), 2.22-2.03(\mathrm{~m}, 4 \mathrm{H}), 1.25(\mathrm{t}, J=7.2 \mathrm{~Hz}, 3 \mathrm{H}) 1.07(\mathrm{~s}, 6 \mathrm{H}) ;{ }^{13} \mathrm{C} \mathrm{NMR}(126 \mathrm{MHz}$, $\left.\mathrm{CDCl}_{3}\right) \delta 174.7,174.3,143.0,142.2,135.7,134.7,126.1,125.9,117.2,116.6,71.6,71.4,60.5$ (overlapped), 49.3 , 49.0, 47.3, 47.2, 46.6, 46.2, 38.5, 38.5, 33.8, 31.5, 29.8, 29.7, 29.7, 14.2, 14.2 (1 peak missing); FT-IR (ATR) v 3470 (br), 3078, 2952, 2866, 2840, 1732, 1715, $1176 \mathrm{~cm}^{-1}$; HRMS (FD) calcd for $\mathrm{C}_{15} \mathrm{H}_{24} \mathrm{O}_{3}\left(\mathrm{M}^{+}\right)$: 252.1725, found: 252.1729 .

\section{$\beta$-Hydroxy ester S20a}

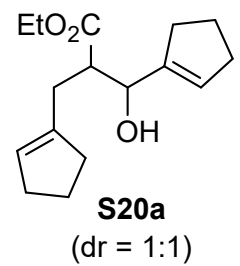

According to procedure A (reaction conditions: $\left.-78^{\circ} \mathrm{C}, 20 \mathrm{~min}\right), i-\operatorname{Pr}_{2} \mathrm{NH}(395 \mu \mathrm{L}$, $2.81 \mathrm{mmol}$ ), $n$-BuLi (2.64 mol/L in hexane, $1.06 \mathrm{~mL}, 2.81 \mathrm{mmol}$ ), ethyl 3(cyclopenten-1-yl)propanoate (394 mg, $2.34 \mathrm{mmol}$ ), and cyclopentene-1carboxaldehyde (270 mg, ca. $2.81 \mathrm{mmol}$ ) gave $\mathbf{S 2 0 a}(\mathrm{dr}=1: 1,472 \mathrm{mg}, 1.79 \mathrm{mmol}$, $76 \%$ yield) as a yellow oil.

S20a: ${ }^{1} \mathrm{H}$ NMR (500 MHz, $\left.\mathrm{CDCl}_{3}\right) \delta 5.69(\mathrm{~s}, 0.48 \mathrm{H}), 5.65(\mathrm{~s}, 0.52 \mathrm{H}), 5.42(\mathrm{~s}, 0.52 \mathrm{H}), 5.37(\mathrm{~s}, 0.48 \mathrm{H})$, $4.49(\mathrm{~d}, J=5.2 \mathrm{~Hz}, 0.48 \mathrm{H}), 4.33(\mathrm{~d}, J=5.7 \mathrm{~Hz}, 0.52 \mathrm{H}), 4.16-4.08(\mathrm{~m}, 2 \mathrm{H}), 2.84-2.78(\mathrm{~m}, 1 \mathrm{H}), 2.54$ $2.46(\mathrm{~m}, 1 \mathrm{H}), 2.40-2.17(\mathrm{~m}, 9 \mathrm{H}), 1.92-1.81(\mathrm{~m}, 4 \mathrm{H}), 1.23(\mathrm{t}, J=7.2 \mathrm{~Hz}, 3 \mathrm{H}) ;{ }^{13} \mathrm{C} \mathrm{NMR}(126 \mathrm{MHz}$, $\left.\mathrm{CDCl}_{3}\right) \delta 175.1,174.7,144.7,143.7,141.9,141.0,127.4,127.3,126.0,125.3,72.1,71.8,60.4$ (overlapped), 48.4, 48.0, 34.9, 34.8, 32.4, 32.4, 32.2, 32.1, 31.5, 31.3, 31.1, 28.7, 23.4, 23.4, 23.4, 23.3, 14.2, 14.2; FT-IR (ATR) v 3448 (br), 3044, 2950, 2845, 1732, 1715, 1157, $1026 \mathrm{~cm}^{-1}$; HRMS (FD) calcd for $\mathrm{C}_{16} \mathrm{H}_{24} \mathrm{O}_{3}\left(\mathrm{M}^{+}\right)$: 264.1725, found: 264.1726 . 


\section{Procedure B: methanesulfonylation and $\beta$-elimination}

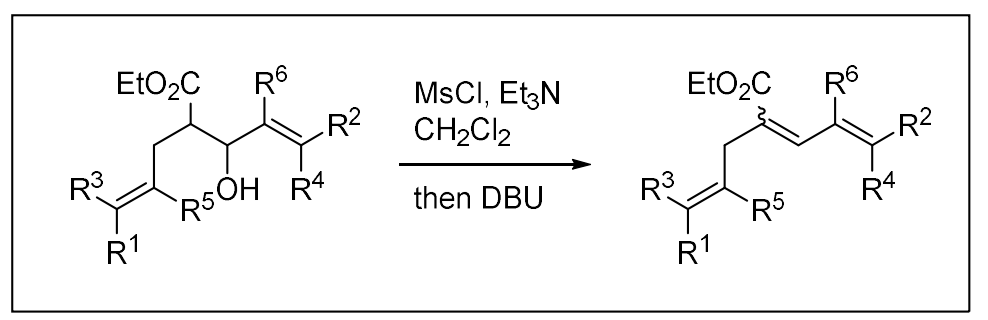

To a solution of $\beta$-hydroxy ester in $\mathrm{CH}_{2} \mathrm{Cl}_{2}\left(0.2 \mathrm{~mol} / \mathrm{L}\right.$ ) were added $\mathrm{Et}_{3} \mathrm{~N}$ (3.0 equiv.) and $\mathrm{MsCl}(2.0$ equiv.) successively at $0{ }^{\circ} \mathrm{C}$. After stirring for $30 \mathrm{~min}$, DBU (5.0 equiv.) was added. The reaction mixture was then warmed up to room temperature and stirred overnight. (For some substrates, the reactions were performed at $50^{\circ} \mathrm{C}$ in 1,2-dichloroethane.) The reaction was quenched with saturated aqueous $\mathrm{NaHCO}_{3}$ solution. Two layers were separated, and the aqueous layer was extracted with EtOAc (3 times). The combined organic layer was dried over $\mathrm{MgSO}_{4}$, filtered, and concentrated under reduced pressure. The resulting residue was purified by flash column chromatography $\left(\mathrm{SiO}_{2}\right.$, hexane:EtOAc $=20: 1)$ to afford the product.

\section{Trienyl ester $4 a$}

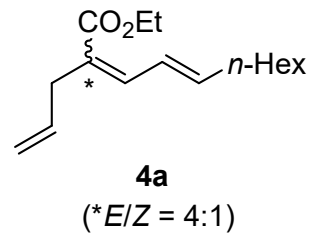

According to procedure B (reaction conditions: rt, 14 h), 3a (1.33 g, 4.96 $\mathrm{mmol}), \mathrm{Et}_{3} \mathrm{~N}(2.08 \mathrm{~mL}, 14.9 \mathrm{mmol}), \mathrm{MsCl}(772 \mu \mathrm{L}, 9.92 \mathrm{mmol})$, and $\mathrm{DBU}(3.70$ $\mathrm{mL}, 24.8 \mathrm{mmol})$ gave $4 \mathrm{a}(E / Z=4: 1,1.21 \mathrm{~g}, 4.82 \mathrm{mmol}, 97 \%$ yield $)$ as a $\left({ }^{*} E / Z=4: 1\right)$ colorless oil.

4a: ${ }^{1} \mathrm{H}$ NMR (500 MHz, $\left.\mathrm{CDCl}_{3}\right) \delta 7.23(\mathrm{~d}, J=10.9 \mathrm{~Hz}, 0.8 \mathrm{H}), 7.10-7.05(\mathrm{~m}, 0.2 \mathrm{H}), 6.39-6.29(\mathrm{~m}$, $1 \mathrm{H}), 6.11(\mathrm{dt}, J=14.7,7.3 \mathrm{~Hz}, 0.8 \mathrm{H}), 5.95(\mathrm{dt}, J=15.0,7.4 \mathrm{~Hz}, 0.2 \mathrm{H}), 5.87-5.79(\mathrm{~m}, 1 \mathrm{H}), 5.08-$ $4.98(\mathrm{~m}, 2 \mathrm{H}), 4.25-4.18(\mathrm{~m}, 2 \mathrm{H}), 3.16(\mathrm{~d}, J=5.7 \mathrm{~Hz}, 0.8 \times 2 \mathrm{H}), 3.04(\mathrm{~d}, J=6.3 \mathrm{~Hz}, 0.2 \times 2 \mathrm{H})$, 2.20-2.14 (m, 2H), 1.45-1.39 (m, 2H), 1.33-1.28 (m, 9H), 0.90-0.87 (m, 3H); ${ }^{13} \mathrm{C}$ NMR (126 MHz, $\left.\mathrm{CDCl}_{3}\right) \delta 168.0,167.3,144.3,142.9,140.8,139.8,136.1,135.6,127.4,126.9,126.6,125.6,116.1$, $115.1,60.4,60.2,38.2,33.3,33.0,31.7,31.6,30.8,28.9,28.8,28.8,22.6,14.3,14.2,14.0$ (overlapped) (2 peaks missing); FT-IR (ATR) v 2956, 2926, 2856, 1703, 1638, $1205 \mathrm{~cm}^{-1}$; HRMS (FD) calcd for $\mathrm{C}_{16} \mathrm{H}_{26} \mathrm{O}_{2}\left(\mathrm{M}^{+}\right)$: 250.1933, found: 250.1943 . 


\section{Trienyl ester $4 b$}

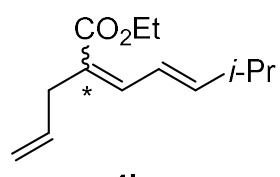

4b

$\left({ }^{*} E / Z=8: 1\right)$

According to procedure B (reaction conditions: rt, $18 \mathrm{~h}), \mathbf{3 b}(1.04 \mathrm{~g}, 4.58 \mathrm{mmol})$, $\mathrm{Et}_{3} \mathrm{~N}(1.91 \mathrm{~mL}, 13.7 \mathrm{mmol}), \mathrm{MsCl}(714 \mu \mathrm{L}, 9.16 \mathrm{mmol})$, and $\mathrm{DBU}(3.42 \mathrm{~mL}$, $22.9 \mathrm{mmol})$ gave $\mathbf{4 b}(E / Z=8: 1,925 \mathrm{mg}, 4.44 \mathrm{mmol}, 97 \%$ yield $)$ as a colorless oil.

4b: ${ }^{1} \mathrm{H}$ NMR (500 MHz, $\left.\mathrm{CDCl}_{3}\right) \delta 7.23(\mathrm{~d}, J=11.6 \mathrm{~Hz}, 0.89 \mathrm{H}), 7.06(\mathrm{dd}, J=15.5,11.2 \mathrm{~Hz}, 0.11 \mathrm{H})$, $6.38(\mathrm{~d}, J=11.2 \mathrm{~Hz}, 0.11 \mathrm{H}), 6.29(\mathrm{dd}, J=14.6,11.6 \mathrm{~Hz}, 0.89 \mathrm{H}), 6.08(\mathrm{q}, J=7.3 \mathrm{~Hz}, 0.89 \mathrm{H}), 5.92$ $(\mathrm{dd}, J=15.2,6.5 \mathrm{~Hz}, 0.11 \mathrm{H}), 5.87-5.79(\mathrm{~m}, 1 \mathrm{H}), 5.08-4.98(\mathrm{~m}, 2 \mathrm{H}), 4.25-4.18(\mathrm{~m}, 2 \mathrm{H}), 3.16(\mathrm{~d}, J$ $=6.3 \mathrm{~Hz}, 0.89 \times 2 \mathrm{H}), 3.04(\mathrm{~d}, J=6.5 \mathrm{~Hz}, 0.11 \times 2 \mathrm{H}), 2.47-2.40(\mathrm{~m}, 1 \mathrm{H}), 1.33-1.28(\mathrm{~m}, 3 \mathrm{H}), 1.04$ $(\mathrm{t}, J=6.0 \mathrm{~Hz}, 6 \mathrm{H}) ;{ }^{13} \mathrm{C} \mathrm{NMR}$ for $(E)-4 \mathbf{b}\left(126 \mathrm{MHz}, \mathrm{CDCl}_{3}\right) \delta 167.9,150.8,139.9,135.6,127.1$, 122.7, 115.1, 60.4, 31.8, 30.9, 21.9 (2C), 14.3; FT-IR (ATR) v 3080, 2961, 2871, 1703, 1638, 1267, $1206 \mathrm{~cm}^{-1}$; HRMS (FD) calcd for $\mathrm{C}_{13} \mathrm{H}_{20} \mathrm{O}_{2}\left(\mathrm{M}^{+}\right)$: 208.1463, found: 208.1470.

\section{Trienyl ester S21a}

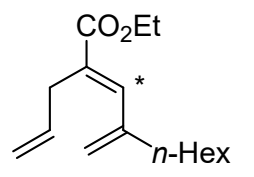

S21a

$\left({ }^{*}(E)\right.$-only $)$

According to procedure B (reaction conditions: rt, 17 h), S19a (192 mg, $716 \mu \mathrm{mol})$, $\mathrm{Et}_{3} \mathrm{~N}(299 \mu \mathrm{L}, 2.15 \mathrm{mmol}), \mathrm{MsCl}(112 \mu \mathrm{L}, 1.43 \mathrm{mmol})$, and DBU $(534 \mu \mathrm{L}, 3.58$ mmol) gave $\mathbf{S 2 1 a}(167 \mathrm{mg}, 667 \mu \mathrm{mol}, 93 \%$ yield) as a colorless oil.

S21a: ${ }^{1} \mathrm{H}$ NMR $\left(500 \mathrm{MHz}, \mathrm{CDCl}_{3}\right) \delta 7.15(\mathrm{~s}, 1 \mathrm{H}), 5.92-5.84(\mathrm{~m}, 1 \mathrm{H}), 5.12(\mathrm{~d}, J=$ $1.7 \mathrm{~Hz}, 1 \mathrm{H}), 5.07(\mathrm{~s}, 1 \mathrm{H}), 5.03-4.98(\mathrm{~m}, 2 \mathrm{H}), 4.22(\mathrm{q}, J=7.0 \mathrm{~Hz}, 2 \mathrm{H}), 3.20(\mathrm{~d}, J=6.3 \mathrm{~Hz}, 2 \mathrm{H}), 2.15$ $(\mathrm{t}, J=7.7 \mathrm{~Hz}, 2 \mathrm{H}), 1.42-1.37(\mathrm{~m}, 2 \mathrm{H}), 1.32-1.26(\mathrm{~m}, 9 \mathrm{H}), 0.88(\mathrm{t}, J=7.0 \mathrm{~Hz}, 3 \mathrm{H}) ;{ }^{13} \mathrm{C}$ NMR $(126$ $\left.\mathrm{MHz}_{\mathrm{CDCl}}\right) \delta 167.9,144.7,141.4,136.4,130.5,116.0,115.2,60.7,36.7,31.9,31.6,28.8,28.2$, 22.6, 14.2, 14.0; FT-IR (ATR) v 3081, 2956, 2928, 2857, 1712, 1204, $907 \mathrm{~cm}^{-1}$; HRMS (FI) calcd for $\mathrm{C}_{16} \mathrm{H}_{26} \mathrm{O}_{2}\left(\mathrm{M}^{+}\right): 250.1933$, found: 250.1943 .

\section{Trienyl ester S21b}

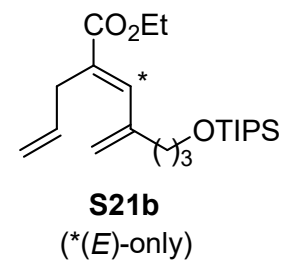

S21b: ${ }^{1} \mathrm{H}$ NMR (500 MHz, $\left.\mathrm{CDCl}_{3}\right) \delta 7.16(\mathrm{~s}, 1 \mathrm{H}), 5.91-5.86(\mathrm{~m}, 1 \mathrm{H}), 5.16(\mathrm{~s}, 1 \mathrm{H}), 5.10(\mathrm{~s}, 1 \mathrm{H}), 5.02-$ $4.98(\mathrm{~m}, 2 \mathrm{H}), 4.21(\mathrm{q}, J=7.3 \mathrm{~Hz}, 2 \mathrm{H}), 3.67(\mathrm{t}, J=6.3 \mathrm{~Hz}, 2 \mathrm{H}), 3.21(\mathrm{~d}, J=5.2 \mathrm{~Hz}, 2 \mathrm{H}), 2.26(\mathrm{t}, J=$
According to procedure $\mathrm{B}$ (reaction conditions: $\mathrm{rt}, 45 \mathrm{~min} ; 50^{\circ} \mathrm{C}, 3 \mathrm{~h}$, in $1,2-$ dichloroethane), S19b (117 mg, $292 \mu \mathrm{mol}), \mathrm{Et}_{3} \mathrm{~N}$ (122 $\left.\mu \mathrm{L}, 877 \mu \mathrm{mol}\right), \mathrm{MsCl}(45.5$ $\mu \mathrm{L}, 584 \mu \mathrm{mol})$, and DBU $(218 \mu \mathrm{L}, 1.46 \mathrm{mmol})$ gave, after purification by flash column chromatography $\left(\mathrm{SiO}_{2}\right.$, hexane:EtOAc $\left.=50: 1\right), \mathbf{S 2 1 b}(88.0 \mathrm{mg}, 231 \mu \mathrm{mol}$, $79 \%$ yield) as a colorless oil. 
$7.7 \mathrm{~Hz}, 2 \mathrm{H}), 1.68-1.62(\mathrm{~m}, 2 \mathrm{H}), 1.30(\mathrm{t}, J=7.3 \mathrm{~Hz}, 3 \mathrm{H}), 1.10-1.03(\mathrm{~m}, 21 \mathrm{H}) ;{ }^{13} \mathrm{C}$ NMR $(126 \mathrm{MHz}$, $\left.\mathrm{CDCl}_{3}\right) \delta 167.9,144.3,141.3,136.3,130.6,116.4,115.3,62.5,60.7,32.9,31.9,31.6,18.0(6 \mathrm{C}), 14.2$, 11.9 (3C); FT-IR (ATR) v 3080, 2942, 2866, 1712, 1206, $1102 \mathrm{~cm}^{-1}$; HRMS (FD) calcd for $\mathrm{C}_{22} \mathrm{H}_{40} \mathrm{O}_{3} \mathrm{Si}$ $\left(\mathrm{M}^{+}\right)$: 380.2747, found: 380.2749 .

\section{Trienyl ester S21c}

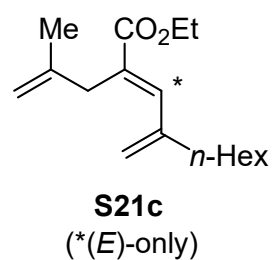

According to procedure B (reaction conditions: rt, 2 d), S19c (248 mg, 878 $\mu \mathrm{mol}), \mathrm{Et}_{3} \mathrm{~N}(367 \mu \mathrm{L}, 2.63 \mathrm{mmol}), \mathrm{MsCl}(137 \mu \mathrm{L}, 1.76 \mathrm{mmol})$, and DBU (655 $\mu \mathrm{L}, 4.39 \mathrm{mmol})$ gave, after purification by flash column chromatography $\left(\mathrm{SiO}_{2}\right.$, hexane:EtOAc = 50:1), S21c $(166 \mathrm{mg}, 628 \mu \mathrm{mol}, 71 \%$ yield $)$ as a colorless oil.

S21c: ${ }^{1} \mathrm{H}$ NMR $\left(500 \mathrm{MHz}, \mathrm{CDCl}_{3}\right) \delta 7.18(\mathrm{~s}, 1 \mathrm{H}), 5.09$ (s, 2H), $4.74(\mathrm{~s}, 1 \mathrm{H}), 4.53(\mathrm{~s}, 1 \mathrm{H}), 4.20(\mathrm{q}, J$ $=7.0 \mathrm{~Hz}, 2 \mathrm{H}), 3.10(\mathrm{~s}, 2 \mathrm{H}), 2.14(\mathrm{t}, J=7.7 \mathrm{~Hz}, 2 \mathrm{H}), 1.77(\mathrm{~s}, 3 \mathrm{H}), 1.42-1.37(\mathrm{~m}, 2 \mathrm{H}), 1.32-1.24(\mathrm{~m}$, $9 \mathrm{H}), 0.88(\mathrm{t}, J=7.0 \mathrm{~Hz}, 3 \mathrm{H}) ;{ }^{13} \mathrm{C}$ NMR $\left(126 \mathrm{MHz}, \mathrm{CDCl}_{3}\right) \delta 168.3,144.7,144.2,141.5,130.6$, 115.9, 109.8, 60.6, 36.8, 35.4, 31.6, 28.8, 28.3, 23.5, 22.6, 14.2, 14.1; FT-IR (ATR) v 3080, 2928, 2856, 1712, 1201, $1096 \mathrm{~cm}^{-1}$; HRMS (FD) calcd for $\mathrm{C}_{17} \mathrm{H}_{28} \mathrm{O}_{2}\left(\mathrm{M}^{+}\right)$: 264.2089, found: 264.2089.

\section{Trienyl ester S21d}

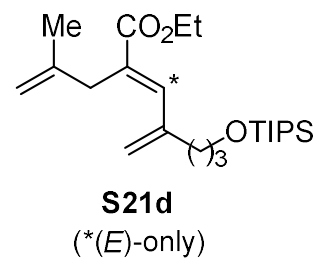

According to procedure $\mathrm{B}$ (reaction conditions: $\mathrm{rt}, 45 \mathrm{~min} ; 50^{\circ} \mathrm{C}, 3 \mathrm{~h}$, in $1,2-$ dichloroethane), S19d (110 mg, $266 \mu \mathrm{mol}), \mathrm{Et}_{3} \mathrm{~N}(111 \mu \mathrm{L}, 797 \mu \mathrm{mol}), \mathrm{MsCl}$ $(83.0 \mu \mathrm{L}, 1.06 \mathrm{mmol})$, and DBU $(199 \mu \mathrm{L}, 1.33 \mathrm{mmol})$ gave, after purification by flash column chromatography $\left(\mathrm{SiO}_{2}\right.$, hexane:EtOAc $\left.=50: 1\right), \mathbf{S 2 1 d}(66.8 \mathrm{mg}$, $169 \mu \mathrm{mol}, 64 \%$ yield) as a yellow oil.

S21d: ${ }^{1} \mathrm{H}$ NMR (500 MHz, $\left.\mathrm{CDCl}_{3}\right) \delta 7.19(\mathrm{~s}, 1 \mathrm{H}), 5.12$ (d, $\left.J=8.6 \mathrm{~Hz}, 2 \mathrm{H}\right), 4.74(\mathrm{~s}, 1 \mathrm{H}), 4.53$ (s, $1 \mathrm{H}), 4.20(\mathrm{q}, J=7.2 \mathrm{~Hz}, 2 \mathrm{H}), 3.67(\mathrm{t}, J=6.3 \mathrm{~Hz}, 2 \mathrm{H}), 3.11(\mathrm{~s}, 2 \mathrm{H}), 2.25(\mathrm{t}, J=7.7 \mathrm{~Hz}, 2 \mathrm{H}), 1.77(\mathrm{~s}$, $3 \mathrm{H}), 1.68-1.62(\mathrm{~m}, 2 \mathrm{H}), 1.29(\mathrm{t}, J=7.2 \mathrm{~Hz}, 3 \mathrm{H}), 1.10-1.03(\mathrm{~m}, 21 \mathrm{H}) ;{ }^{13} \mathrm{C} \mathrm{NMR}\left(126 \mathrm{MHz}, \mathrm{CDCl}_{3}\right)$ $\delta 168.2,144.2,144.1,141.3,130.8,116.2,109.8,62.5,60.7,35.5,33.0,31.6,23.5,18.0$ (6C), 14.2, 12.0 (3C); FT-IR (ATR) v 3080, 2941, 2866, 1713, 1203, 1098, $882 \mathrm{~cm}^{-1}$; HRMS (FD) calcd for $\mathrm{C}_{23} \mathrm{H}_{42} \mathrm{O}_{3} \mathrm{Si}\left(\mathrm{M}^{+}\right)$: 394.2903, found: 394.2916. 


\section{Trienyl ester S22a}

$\mathrm{CO}_{2} \mathrm{Et}$ According to procedure B (reaction conditions: rt, $20 \mathrm{~h}$ ), S15a (215 mg, $\left.957 \mu \mathrm{mol}\right)$, $\mathrm{Et}_{3} \mathrm{~N}(400 \mu \mathrm{L}, 2.87 \mathrm{mmol}), \mathrm{MsCl}(149 \mu \mathrm{L}, 1.91 \mathrm{mmol})$, and $\mathrm{DBU}(713 \mu \mathrm{L}, 4.78 \mathrm{mmol})$ gave $\mathbf{S 2 2 a}$ (193 mg, $936 \mu \mathrm{mol}, 98 \%$ yield) as a yellow oil.

S22a

$\left({ }^{*}(E)\right.$-only)

S22a: ${ }^{1} \mathrm{H}$ NMR $\left(500 \mathrm{MHz}, \mathrm{CDCl}_{3}\right) \delta 7.41(\mathrm{~s}, 1 \mathrm{H}), 6.12(\mathrm{~s}, 1 \mathrm{H}), 5.91-5.83(\mathrm{~m}, 1 \mathrm{H}), 5.02(\mathrm{~d}, J=1.1 \mathrm{~Hz}$, $1 \mathrm{H}), 5.00-4.98(\mathrm{~m}, 1 \mathrm{H}), 4.21$ (q, $J=7.2 \mathrm{~Hz}, 2 \mathrm{H}), 3.25$ (d, $J=5.7 \mathrm{~Hz}, 2 \mathrm{H}), 2.65-2.62(\mathrm{~m}, 2 \mathrm{H}), 2.42$ (td, $J=7.4,2.3 \mathrm{~Hz}, 2 \mathrm{H}), 1.99-1.92(\mathrm{~m}, 2 \mathrm{H}), 1.30(\mathrm{t}, J=7.2 \mathrm{~Hz}, 3 \mathrm{H}) ;{ }^{13} \mathrm{C} \mathrm{NMR}\left(126 \mathrm{MHz}, \mathrm{CDCl}_{3}\right) \delta 168.5$, 140.7, 140.3, 136.5, 127.3, 115.0, 60.6, 33.7, 32.5, 31.3, 24.0, 14.3 (1 peak missing); FT-IR (ATR) $v$ 3080, 2956, 2843, 1703, 1274, $1199 \mathrm{~cm}^{-1}$; HRMS (FI) calcd for $\mathrm{C}_{13} \mathrm{H}_{18} \mathrm{O}_{2}\left(\mathrm{M}^{+}\right)$: 206.1307, found: 206.1302.

\section{Trienyl ester S22b}

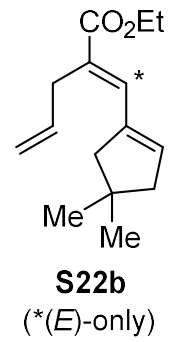

According to procedure B (reaction conditions: rt, $21 \mathrm{~h}$ ), S15b (341 mg, $1.35 \mathrm{mmol})$, $\mathrm{Et}_{3} \mathrm{~N}(564 \mu \mathrm{L}, 4.05 \mathrm{mmol}), \mathrm{MsCl}(210 \mu \mathrm{L}, 2.70 \mathrm{mmol})$, and $\mathrm{DBU}(1.01 \mathrm{~mL}, 6.75 \mathrm{mmol})$ gave $\mathbf{S 2 2 b}$ (300 mg, $1.28 \mathrm{mmol}, 95 \%$ yield) as a colorless oil.

S22b: ${ }^{1} \mathrm{H}$ NMR (500 MHz, $\left.\mathrm{CDCl}_{3}\right) \delta 7.36(\mathrm{~s}, 1 \mathrm{H}), 6.01(\mathrm{~s}, 1 \mathrm{H}), 5.91-5.83(\mathrm{~m}, 1 \mathrm{H}), 5.02-4.98(\mathrm{~m}$, 2H), 4.20 (q, $J=7.0 \mathrm{~Hz}, 2 \mathrm{H}), 3.23$ (d, $J=5.2 \mathrm{~Hz}, 2 \mathrm{H}), 2.45$ (s, 2H), 2.23 (s, 2H), 1.29 (t, $J=7.0 \mathrm{~Hz}$, $3 \mathrm{H}), 1.10(\mathrm{~s}, 6 \mathrm{H}) ;{ }^{13} \mathrm{C} \mathrm{NMR}\left(126 \mathrm{MHz}, \mathrm{CDCl}_{3}\right) \delta 168.5,139.5,139.1,136.9,136.5,127.2,115.1$, 60.6, 48.8, 47.4, 39.3, 31.3, 29.6 (2C), 14.3; FT-IR (ATR) v 2953, 2867, 2836, 1704, 1273, 1203 , $1193 \mathrm{~cm}^{-1}$; HRMS (FD) calcd for $\mathrm{C}_{15} \mathrm{H}_{22} \mathrm{O}_{2}\left(\mathrm{M}^{+}\right): 234.1620$, found: 234.1623 .

\section{Trienyl ester S23a}

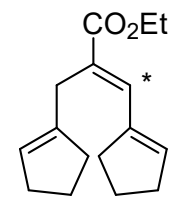

According to procedure B (reaction conditions: rt, $13 \mathrm{~h}), \mathbf{S 2 0 a}(472 \mathrm{mg}, 1.79 \mathrm{mmol})$, $\mathrm{Et}_{3} \mathrm{~N}(747 \mu \mathrm{L}, 5.36 \mathrm{mmol}), \mathrm{MsCl}(278 \mu \mathrm{L}, 3.57 \mathrm{mmol})$, and DBU $(1.33 \mathrm{~mL}, 8.93 \mathrm{mmol})$ gave S23a (398 mg, $1.62 \mathrm{mmol}, 91 \%$ yield) as a colorless oil.

S23a

${ }^{*}(E)$-only)

S23a: ${ }^{1} \mathrm{H}$ NMR (500 MHz, $\left.\mathrm{CDCl}_{3}\right) \delta 7.39$ (s, 1H), 6.08 (s, 1H), 5.20 (s, 1H), 4.19 (q, J= $7.2 \mathrm{~Hz}$, $2 \mathrm{H}), 3.21(\mathrm{~s}, 2 \mathrm{H}), 2.61-2.58(\mathrm{~m}, 2 \mathrm{H}), 2.43-2.39(\mathrm{~m}, 2 \mathrm{H}), 2.26(\mathrm{t}, J=7.4 \mathrm{~Hz}, 4 \mathrm{H}), 1.97-1.91(\mathrm{~m}$, $2 \mathrm{H}), 1.89-1.83(\mathrm{~m}, 2 \mathrm{H}), 1.28(\mathrm{t}, J=7.2 \mathrm{~Hz}, 3 \mathrm{H}) ;{ }^{13} \mathrm{C} \mathrm{NMR}\left(126 \mathrm{MHz}, \mathrm{CDCl}_{3}\right) \delta 168.9,142.9,140.9$, 
139.9, 135.8, 128.1, 124.0, 60.5, 35.6, 33.5, 32.5, 32.3, 29.2, 24.0, 23.3, 14.3; FT-IR (ATR) v 3043, 2951, 2843, 1703, 1275, 1211, $1188 \mathrm{~cm}^{-1}$; HRMS (FD) calcd for $\mathrm{C}_{16} \mathrm{H}_{22} \mathrm{O}_{2}\left(\mathrm{M}^{+}\right): 246.1620$, found: 246.1630 .

\section{Preparation of other trienyl esters (for syntheses of 7c, 14, 16, 20b)}

\section{Trienyl ester $4 c$}

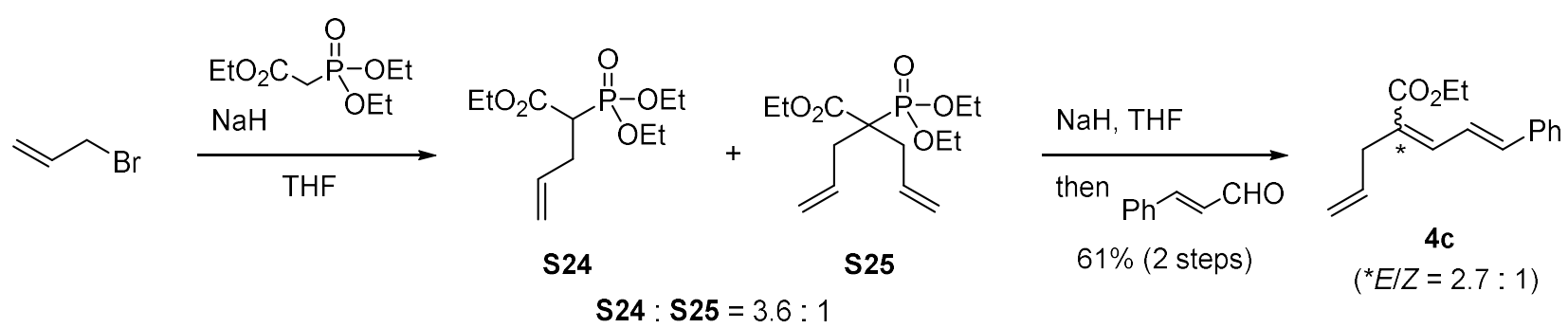

$\mathrm{NaH}$ (55\% dispersion in mineral oil, $266 \mathrm{mg}, 6.10 \mathrm{mmol}$ ) was washed with THF and dissolved with THF (25 mL). To this mixture was added triethyl phosphonoacetate $(1.201 \mathrm{~mL}, 6.00 \mathrm{mmol})$ at $0{ }^{\circ} \mathrm{C}$. After stirring for $30 \mathrm{~min}$, allyl bromide $(423 \mu \mathrm{L}, 5.00 \mathrm{mmol})$ was added. The reaction mixture was then warmed up to room temperature and stirred for $20 \mathrm{~h}$. The reaction was quenched with saturated aqueous $\mathrm{NH}_{4} \mathrm{Cl}$ solution $(25 \mathrm{~mL}$ ). Two layers were separated, and the aqueous layer was extracted with EtOAc $(20 \mathrm{~mL} \times 3)$. The combined organic layer was dried over $\mathrm{MgSO}_{4}$, filtered, and concentrated under reduced pressure. The resulting residue was purified by flash column chromatography $\left(\mathrm{SiO}_{2}\right.$, hexane:EtOAc $\left.=1: 2\right)$ to afford a mixture of $\mathbf{S 2 4}$ and $\mathbf{S 2 5}(\mathbf{S 2 4}: \mathbf{S 2 5}=3.6$ : $1,1.170 \mathrm{~g})$.

$\mathrm{NaH}$ (55\% dispersion in mineral oil, $165 \mathrm{mg}, 3.78 \mathrm{mmol}$ ) was washed with THF and dissolved with THF (15.7 mL). To this mixture was added a mixture of S24 and S25 (S24: S25 = 3.6: 1, 1.170 g) at $0{ }^{\circ} \mathrm{C}$. After stirring for $30 \mathrm{~min}$, cinnamaldehyde $(475 \mu \mathrm{L}, 3.78 \mathrm{mmol})$ was added. The reaction mixture was then warmed up to room temperature and stirred for $30 \mathrm{~min}$. The reaction was quenched with saturated aqueous $\mathrm{NH}_{4} \mathrm{Cl}$ solution $(15 \mathrm{~mL})$. Two layers were separated, and the aqueous layer was extracted with EtOAc $(15 \mathrm{~mL} \times 3)$. The combined organic layer was dried over $\mathrm{MgSO}_{4}$, filtered, and concentrated under reduced pressure. The resulting residue was purified by flash column chromatography $\left(\mathrm{SiO}_{2}\right.$, hexane:EtOAc $\left.=50: 1\right)$ to afford $4 \mathrm{c}(E / Z=2.7: 1,738 \mathrm{mg}, 3.05 \mathrm{mmol}, 61 \%$ yield for 2 steps) as a yellow oil.

4c: ${ }^{1} \mathrm{H}$ NMR $\left(500 \mathrm{MHz}, \mathrm{CDCl}_{3}\right) \delta 7.89(\mathrm{dd}, J=15.5,11.2 \mathrm{~Hz}, 0.27 \mathrm{H}), 7.48-7.26(\mathrm{~m}, 5 \mathrm{H}+0.73 \mathrm{H}), 7.05$ $(\mathrm{dd}, J=15.2,11.2 \mathrm{~Hz}, 0.73 \mathrm{H}), 6.90(\mathrm{~d}, J=15.5 \mathrm{~Hz}, 0.73 \mathrm{H}), 6.73(\mathrm{~d}, J=15.5 \mathrm{~Hz}, 0.27 \mathrm{H}), 6.58(\mathrm{~d}, J=$ $11.2 \mathrm{~Hz}, 0.27 \mathrm{H}), 5.93-5.84(\mathrm{~m}, 1 \mathrm{H}), 5.12-5.02(\mathrm{~m}, 2 \mathrm{H}), 4.30-4.22(\mathrm{~m}, 2 \mathrm{H}), 3.28(\mathrm{~d}, J=5.7 \mathrm{~Hz}, 0.73 \times$ $2 \mathrm{H}), 3.13(\mathrm{~d}, J=6.3 \mathrm{~Hz}, 0.27 \times 2 \mathrm{H}), 1.36(\mathrm{t}, J=7.2 \mathrm{~Hz}, 0.27 \times 3 \mathrm{H}), 1.33(\mathrm{t}, J=6.9 \mathrm{~Hz}, 0.73 \times 3 \mathrm{H}) ;{ }^{13} \mathrm{C}$ NMR (126 MHz, $\left.\mathrm{CDCl}_{3}\right) \delta 167.7,167.1,140.3,139.8,139.3,138.7,136.8,136.4,135.8,135.6,129.2$, 
129.0, $128.8(2 \mathrm{C}), 128.7$ (2C), $128.6(2 \mathrm{C}), 128.4,127.1$ (2C), 125.8, 123.6, 116.4, 115.4, 60.6, 60.3, 38.4, 31.1, 14.3, 14.3 (1 peak missing); FT-IR (ATR) v 3079, 2979, 2932, 2904, 1698, 1620, 1201, 747, $689 \mathrm{~cm}^{-1}$; HRMS (FD) calcd for $\mathrm{C}_{16} \mathrm{H}_{18} \mathrm{O}_{2}\left(\mathrm{M}^{+}\right)$: 242.1307, found: 242.1297.

\section{Trienyl ester S27}

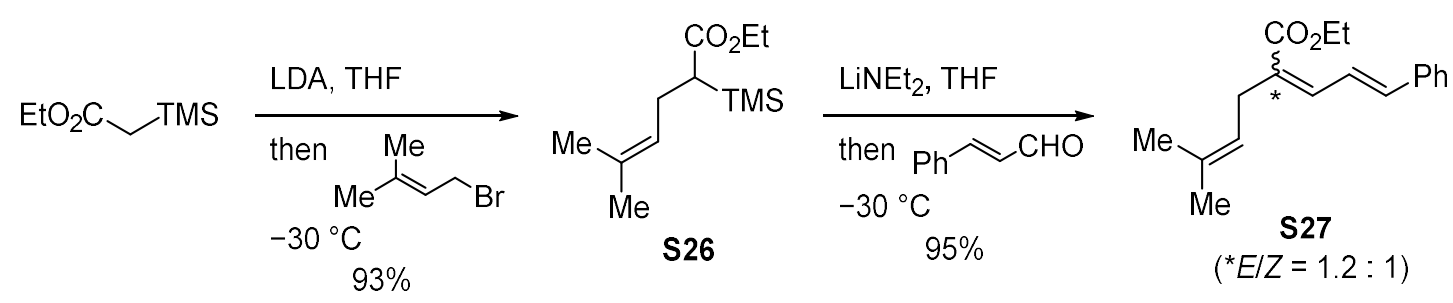

\section{S26}

To a solution of $i$ - $\operatorname{Pr}_{2} \mathrm{NH}(843 \mu \mathrm{L}, 6.00 \mathrm{mmol})$ in THF $(25 \mathrm{~mL})$ was added $n$-BuLi $(2.67 \mathrm{~mol} / \mathrm{L}$ in hexane, $2.25 \mathrm{~mL}, 6.00 \mathrm{mmol}$ ) at $0{ }^{\circ} \mathrm{C}$ and the reaction mixture was stirred for $30 \mathrm{~min}$. The reaction mixture was then cooled down to $-78^{\circ} \mathrm{C}$ and ethyl (trimethylsilyl)acetate $(911 \mu \mathrm{L}, 5.00 \mathrm{mmol}$ ) was added. After stirring for $30 \mathrm{~min}, 1$-bromo-3-methyl-2-butene ( $693 \mu \mathrm{L}, 6.00 \mathrm{mmol})$ was added. The reaction mixture was then warmed up to $-30{ }^{\circ} \mathrm{C}$ and stirred for $30 \mathrm{~min}$. The reaction was quenched with saturated aqueous $\mathrm{NH}_{4} \mathrm{Cl}$ solution. Two layers were separated, and the aqueous layer was extracted with EtOAc (3 times). The combined organic layer was dried over $\mathrm{MgSO}_{4}$, filtered, and concentrated under reduced pressure. The resulting residue was purified by flash column chromatography $\left(\mathrm{SiO}_{2}\right.$, hexane:EtOAc $\left.=50: 1\right)$ to afford $\mathbf{S 2 6}(1.07 \mathrm{~g}, 4.68 \mathrm{mmol}, 93 \%$ yield $)$ as a colorless oil.

S26: ${ }^{1} \mathrm{H}$ NMR $\left(500 \mathrm{MHz}, \mathrm{CDCl}_{3}\right) \delta 5.08(\mathrm{t}, J=6.6 \mathrm{~Hz}, 1 \mathrm{H}), 4.13-4.06(\mathrm{~m}, 2 \mathrm{H}), 2.47-2.41(\mathrm{~m}, 1 \mathrm{H})$, 2.13-2.08 (m, 1H), $1.96(\mathrm{dd}, J=11.2,3.7 \mathrm{~Hz}, 1 \mathrm{H}), 1.66(\mathrm{~s}, 3 \mathrm{H}), 1.61(\mathrm{~s}, 3 \mathrm{H}), 1.23$ (t, $J=6.9 \mathrm{~Hz}$, $3 \mathrm{H}), 0.08(\mathrm{~s}, 9 \mathrm{H}) ;{ }^{13} \mathrm{C} \mathrm{NMR}\left(126 \mathrm{MHz}, \mathrm{CDCl}_{3}\right) \delta 175.2,132.0,124.1,59.6,38.3,25.7,25.2,17.7$, 14.5, -2.6 (3C); FT-IR (ATR) $v$ 2963, 2914, 1716, 1250, 1140, $840 \mathrm{~cm}^{-1}$; HRMS (FI) calcd for $\mathrm{C}_{12} \mathrm{H}_{24} \mathrm{O}_{2} \mathrm{Si}\left(\mathrm{M}^{+}\right)$: 228.1546, found: 228.1543 .

\section{S27}

To a solution of $\mathrm{Et}_{2} \mathrm{NH}(373 \mu \mathrm{L}, 3.60 \mathrm{mmol})$ in THF $(15 \mathrm{~mL})$ was added $n$-BuLi $(2.67 \mathrm{~mol} / \mathrm{L}$ in hexane, $1.35 \mathrm{~mL}, 3.60 \mathrm{mmol}$ ) at $0{ }^{\circ} \mathrm{C}$ and the reaction mixture was stirred for $30 \mathrm{~min}$. The reaction mixture was then cooled down to $-78^{\circ} \mathrm{C}$ and $\mathbf{S 2 6}$ (686 $\left.\mathrm{mg}, 3.00 \mathrm{mmol}\right)$ in THF (1.0 mL) was added. After stirring for $30 \mathrm{~min}$, cinnamaldehyde $(566 \mu \mathrm{L}, 3.60 \mathrm{mmol})$ was added. The reaction mixture was then warmed up to $-30{ }^{\circ} \mathrm{C}$ and stirred for $1 \mathrm{~h}$. The reaction was quenched with saturated aqueous $\mathrm{NH}_{4} \mathrm{Cl}$ solution. Two layers were separated, and the aqueous layer was extracted with EtOAc (3 times). The combined organic layer was dried over $\mathrm{MgSO}_{4}$, filtered, and concentrated under reduced pressure. The resulting residue was purified by flash column chromatography $\left(\mathrm{SiO}_{2}\right.$, hexane:EtOAc 
$=50: 1)$ to afford $\mathbf{S 2 7}(E / Z=1.2: 1,773 \mathrm{mg}, 2.86 \mathrm{mmol}, 95 \%$ yield $)$ as a yellow oil.

S27: ${ }^{1} \mathrm{H}$ NMR $\left(500 \mathrm{MHz}, \mathrm{CDCl}_{3}\right) \delta 7.86(\mathrm{dd}, J=15.7,11.1 \mathrm{~Hz}, 0.45 \mathrm{H}), 7.48-7.45(\mathrm{~m}, 2 \mathrm{H}), 7.37-$ $7.24(\mathrm{~m}, 3 \mathrm{H}+0.55 \mathrm{H}), 7.09(\mathrm{dd}, J=15.5,11.5 \mathrm{~Hz}, 0.55 \mathrm{H}), 6.88(\mathrm{~d}, J=15.5 \mathrm{~Hz}, 0.55 \mathrm{H}), 6.71(\mathrm{~d}, J$ $=15.7 \mathrm{~Hz}, 0.45 \mathrm{H}), 6.54(\mathrm{~d}, J=11.1 \mathrm{~Hz}, 0.45 \mathrm{H}), 5.18(\mathrm{t}, J=7.2 \mathrm{~Hz}, 0.45 \mathrm{H}), 5.08(\mathrm{t}, J=6.9 \mathrm{~Hz}$, $0.55 \mathrm{H}), 4.30-4.21(\mathrm{~m}, 2 \mathrm{H}), 3.22(\mathrm{~d}, J=6.9 \mathrm{~Hz}, 0.55 \times 2 \mathrm{H}), 3.06(\mathrm{~d}, J=7.2 \mathrm{~Hz}, 0.45 \times 2 \mathrm{H}), 1.78(\mathrm{~s}$, $0.55 \times 3 \mathrm{H}), 1.75(\mathrm{~s}, 0.45 \times 3 \mathrm{H}), 1.70(\mathrm{~s}, 0.55 \times 3 \mathrm{H}), 1.67(\mathrm{~s}, 0.45 \times 3 \mathrm{H}), 1.37(\mathrm{t}, J=7.2 \mathrm{~Hz}, 0.45 \times$ $3 \mathrm{H}), 1.33(\mathrm{t}, J=7.2 \mathrm{~Hz}, 0.55 \times 3 \mathrm{H}) ;{ }^{13} \mathrm{C} \mathrm{NMR}\left(126 \mathrm{MHz}, \mathrm{CDCl}_{3}\right) \delta 168.0,167.6,139.2,139.0$, 138.1, 138.0, 136.9, 136.6, 133.8, 132.3, 131.3, 130.3, 128.7 (2C), 128.7 (2C), 128.6 (2C), 128.2, 127.0 (2C), 126.0, 123.8, 121.9, 121.1, 60.6, 60.3, 32.8, 26.3, 25.8, 25.7, 17.9, 17.8, 14.3, 14.3 (1 peak missing); FT-IR (ATR) v 2979, 2911, 1699, 1220, 1173, 746, $689 \mathrm{~cm}^{-1}$; HRMS (FD) calcd for $\mathrm{C}_{18} \mathrm{H}_{22} \mathrm{O}_{2}\left(\mathrm{M}^{+}\right): 270.1620$, found: 270.1612 .

\section{Trienyl ester S28}

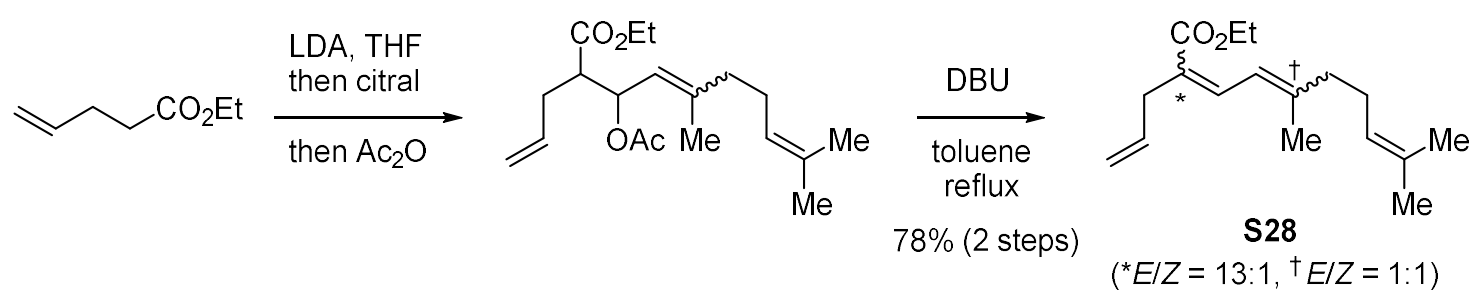

To a solution of $i$ - $\operatorname{Pr}_{2} \mathrm{NH}(169 \mu \mathrm{L}, 1.20 \mathrm{mmol})$ in THF $(5.0 \mathrm{~mL})$ was added $n$-BuLi $(2.69 \mathrm{~mol} / \mathrm{L}$ in hexane, $449 \mu \mathrm{L}, 1.20 \mathrm{mmol}$ ) at $0{ }^{\circ} \mathrm{C}$, and the reaction mixture was stirred for $30 \mathrm{~min}$. The resulting mixture was then cooled down to $-78^{\circ} \mathrm{C}$ and ethyl 4-pentenoate $(142 \mu \mathrm{L}, 1.00 \mathrm{mmol})$ was added. After stirring for $30 \mathrm{~min}$, citral (cis and trans mixture, $205 \mu \mathrm{L}, 1.20 \mathrm{mmol}$ ) was added and the reaction mixture was stirred for $15 \mathrm{~min}$ at the same temperature before $\mathrm{Ac}_{2} \mathrm{O}(189 \mu \mathrm{L}, 2.00 \mathrm{mmol})$ was added. After stirring for additional $15 \mathrm{~min}$, the reaction was quenched with saturated aqueous $\mathrm{NH}_{4} \mathrm{Cl}$ solution $(5.0 \mathrm{~mL})$. Two layers were separated, and the aqueous layer was extracted with EtOAc $(5.0 \mathrm{~mL} \times 3)$. The combined organic layer was dried over $\mathrm{MgSO}_{4}$, filtered, and concentrated under reduced pressure to afford a crude oil.

To a solution of the crude ester in toluene $(5.0 \mathrm{~mL})$ was added DBU $(746 \mu \mathrm{L}, 5.00 \mathrm{mmol})$ at room temperature. The reaction mixture was then warmed up to $130{ }^{\circ} \mathrm{C}$ and refluxed for $2.5 \mathrm{~h}$. The reaction was quenched with saturated aqueous $\mathrm{NaHCO}_{3}$ solution $(5.0 \mathrm{~mL})$. Two layers were separated, and the aqueous layer was extracted with EtOAc $(5.0 \mathrm{~mL} \times 3)$. The combined organic layer was dried over $\mathrm{MgSO}_{4}$, filtered, and concentrated under reduced pressure. The resulting residue was purified by flash column chromatography $\left(\mathrm{SiO}_{2}\right.$, hexane:EtOAc $\left.=50: 1\right)$ to afford $\mathbf{S 2 8}(2 E / 2 Z=13: 1,4 E / 4 Z$ $=1: 1,205 \mathrm{mg}, 781 \mu \mathrm{mol}, 78 \%$ yield for 2 steps) as a yellow oil ${ }^{[8]}$.

S28: ${ }^{1} \mathrm{H}$ NMR for $(2 E, 4 E)-S 28$ and $(2 E, 4 Z)-\mathbf{S 2 8}\left(500 \mathrm{MHz}, \mathrm{CDCl}_{3}\right) \delta 7.56-7.52(\mathrm{~m}, 1 \mathrm{H}), 6.13(\mathrm{~s}$, $0.5 \mathrm{H}), 6.11(\mathrm{~s}, 0.5 \mathrm{H}), 5.87-5.79(\mathrm{~m}, 1 \mathrm{H}), 5.14-4.97(\mathrm{~m}, 3 \mathrm{H}), 4.24-4.20(\mathrm{~m}, 2 \mathrm{H}), 3.16(\mathrm{~d}, J=6.3 \mathrm{~Hz}$, 
2H), 2.32-2.10 (m, 4H), $1.90(\mathrm{~s}, 3 \mathrm{H}), 1.68(\mathrm{~s}, 3 \mathrm{H}), 1.62(\mathrm{~s}, 0.5 \times 3 \mathrm{H}) 1.61(\mathrm{~s}, 0.5 \times 3 \mathrm{H}), 1.31-1.28$ $(\mathrm{m}, 3 \mathrm{H}) ;{ }^{13} \mathrm{C}$ NMR for $(2 E, 4 E)-\mathbf{S 2 8}$ and $(2 E, 4 Z)-\mathbf{S 2 8}\left(126 \mathrm{MHz}, \mathrm{CDCl}_{3}\right) \delta 168.3$ (overlapped), 149.1, $148.9,135.8,135.7,135.4,135.1,132.5,132.1,126.5,126.2,123.4,123.4,121.1,120.4,115.0$, 115.0, 60.4, 60.4, 40.6, 32.8, 30.7, 30.6, 26.9, 26.3, 25.7, 24.9, 17.7, 17.6, 17.4, 14.3 (overlapped) (1 peak missing); FT-IR (ATR) v 2977, 2915, 2856, 1702, 1633, 1263, $1199 \mathrm{~cm}^{-1}$; HRMS (FD) calcd for $\mathrm{C}_{17} \mathrm{H}_{26} \mathrm{O}_{2}\left(\mathrm{M}^{+}\right)$: 262.1933, found: 262.1941 .

\section{Trienyl ester S23b}
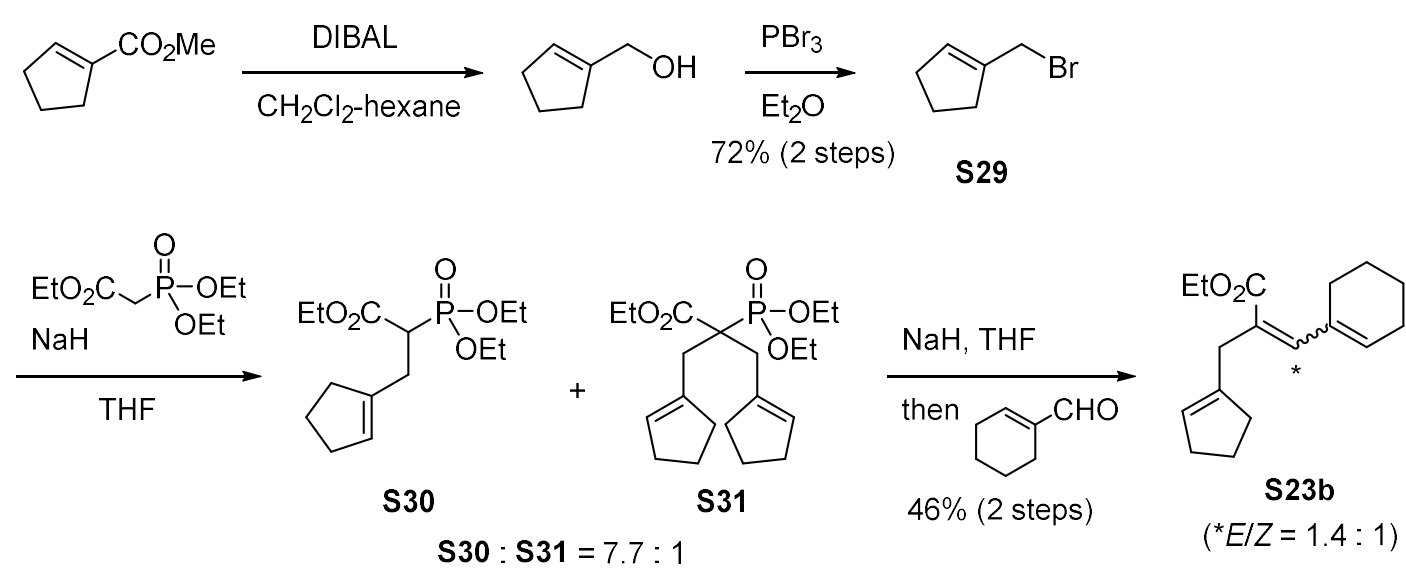

\section{S29}

To a solution of methyl cyclopentene-1-carboxylate $(612 \mu \mathrm{L}, 5.00 \mathrm{mmol})$ in $\mathrm{CH}_{2} \mathrm{Cl}_{2}(25 \mathrm{~mL})$ was added DIBAL $(1.03 \mathrm{~mol} / \mathrm{L}$ in hexane, $10.7 \mathrm{~mL}, 11.0 \mathrm{mmol})$ at $-78^{\circ} \mathrm{C}$. After stirring for $15 \mathrm{~min}$, the reaction was quenched with saturated aqueous Rochelle salt (potassium sodium $(+)$-tartrate) solution $(30 \mathrm{~mL})$, and the biphasic mixture was stirred vigorously overnight. The two layers were separated, and the aqueous layer was extracted with $\mathrm{CH}_{2} \mathrm{Cl}_{2}(20 \mathrm{~mL} \times 3)$. The combined organic layer was dried over $\mathrm{MgSO}_{4}$, filtered, and concentrated under reduced pressure to afford a crude oil.

To a solution of the crude alcohol in $\mathrm{Et}_{2} \mathrm{O}(25 \mathrm{~mL})$ was added $\mathrm{PBr}_{3}(237 \mu \mathrm{L}, 2.50 \mathrm{mmol})$ at $0{ }^{\circ} \mathrm{C}$. After stirring for $30 \mathrm{~min}$, the reaction mixture was warmed up to room temperature and stirred for $21 \mathrm{~h}$. The reaction was quenched with saturated aqueous $\mathrm{NaHCO}_{3}$ solution $(20 \mathrm{~mL})$, two layers were separated, and the aqueous layer was extracted with $\mathrm{Et}_{2} \mathrm{O}(20 \mathrm{~mL} \times 3)$. The combined organic layer was dried over $\mathrm{MgSO}_{4}$, filtered, and concentrated under reduced pressure. The resulting residue was purified by flash column chromatography $\left(\mathrm{SiO}_{2}\right.$, pentane: $\left.\mathrm{Et}_{2} \mathrm{O}=20: 1\right)$ to afford $\mathbf{S 2 9}(579 \mathrm{mg}, 3.60$ mmol, $72 \%$ for 2 steps) as a colorless oil.

S29: ${ }^{1} \mathrm{H}$ spectral property was identical to that reported ${ }^{[7]}$. 


\section{S23b}

$\mathrm{NaH}$ (55\% dispersion in mineral oil, $77.8 \mathrm{mg}, 1.78 \mathrm{mmol}$ ) was washed with THF and dissolved with THF $(7.5 \mathrm{~mL})$. To this mixture was added triethyl phosphonoacetate $(360 \mu \mathrm{L}, 1.80 \mathrm{mmol})$ at $0{ }^{\circ} \mathrm{C}$. After stirring for $30 \mathrm{~min}$, $\mathbf{S 2 9}(242 \mathrm{mg}, 1.50 \mathrm{mmol})$ in THF $(0.5 \mathrm{~mL})$ was added. The reaction mixture was then warmed up to room temperature and stirred for $23 \mathrm{~h}$. The reaction was quenched with saturated aqueous $\mathrm{NH}_{4} \mathrm{Cl}$ solution $(7.5 \mathrm{~mL})$. Two layers were separated, and the aqueous layer was extracted with EtOAc $(7.5 \mathrm{~mL} \times 3)$. The combined organic layer was dried over $\mathrm{MgSO}_{4}$, filtered, and concentrated under reduced pressure. The resulting residue was purified by flash column chromatography $\left(\mathrm{SiO}_{2}\right.$, hexane:EtOAc $\left.=1: 1\right)$ to afford a mixture of $\mathbf{S 3 0}$ and $\mathbf{S 3 1}(\mathbf{S 3 0}: \mathbf{S 3 1}=7.7$ : $1,385 \mathrm{mg})$.

$\mathrm{NaH}(55 \%$ dispersion in mineral oil, $26.2 \mathrm{mg}, 600 \mu \mathrm{mol})$ was washed with THF and dissolved with THF (2.5 mL). To this mixture was added a mixture of $\mathbf{S 3 0}$ and $\mathbf{S 3 1}$ (S30 : S31 = $7.7: 1,177 \mathrm{mg})$ in THF $(0.5 \mathrm{~mL})$ at $0{ }^{\circ} \mathrm{C}$. After stirring for $30 \mathrm{~min}$, cyclohexene-1-carboxaldehyde $(68.4 \mu \mathrm{L}, 600$ $\mu \mathrm{mol})$ was added. The reaction mixture was then warmed up to room temperature and stirred for 3.5 h. The reaction was quenched with saturated aqueous $\mathrm{NH}_{4} \mathrm{Cl}$ solution $(2.5 \mathrm{~mL})$. Two layers were separated, and the aqueous layer was extracted with EtOAc $(2.5 \mathrm{~mL} \times 3)$. The combined organic layer was dried over $\mathrm{MgSO}_{4}$, filtered, and concentrated under reduced pressure. The resulting residue was purified by flash column chromatography $\left(\mathrm{SiO}_{2}\right.$, hexane:EtOAc $\left.=50: 1\right)$ to afford $\mathbf{S 2 3 b}$ $(E / Z=1.4: 1,82.8 \mathrm{mg}, 318 \mu \mathrm{mol}, 46 \%$ yield for 2 steps $)$ as a colorless oil.

S23b: ${ }^{1} \mathrm{H}$ NMR (500 MHz, $\left.\mathrm{CDCl}_{3}\right) \delta 7.11(\mathrm{~s}, 0.58 \mathrm{H}), 6.00(\mathrm{~s}, 0.42 \mathrm{H}), 5.96(\mathrm{~s}, 0.58 \mathrm{H}), 5.75(\mathrm{~s}, 0.42 \mathrm{H})$, $5.41(\mathrm{~s}, 0.42 \mathrm{H}), 5.20(\mathrm{~s}, 0.58 \mathrm{H}), 4.21-4.15(\mathrm{~m}, 2 \mathrm{H}), 3.19(\mathrm{~s}, 0.58 \times 2 \mathrm{H}), 3.04(\mathrm{~s}, 0.42 \times 2 \mathrm{H}), 2.30-$ 2.09 (m, 8H), 1.89-1.82 (m, 2H), 1.66-1.48 (m, 4H), 1.29-1.25 (m, 3H); ${ }^{13} \mathrm{C}$ NMR (126 MHz, $\left.\mathrm{CDCl}_{3}\right) \delta 170.3,169.0,143.1,142.4,141.4,136.0,134.8,134.7,133.5,131.0,128.6,127.5,126.3$, 123.8, 60.4, 60.3, 37.6, 35.6, 34.6, 32.5, 32.4, 29.5, 27.8, 26.5, 26.1, 25.9, 23.5, 23.3, 22.6, 22.6, 21.9, 21.7, 14.2, 14.1; FT-IR (ATR) v 2978, 2930, 2844, 1706, 1213, $1183 \mathrm{~cm}^{-1}$; HRMS (FI) calcd for $\mathrm{C}_{17} \mathrm{H}_{24} \mathrm{O}_{2}\left(\mathrm{M}^{+}\right): 260.1776$, found: 260.1773 . 


\section{Preparation of Phosphate 6a}

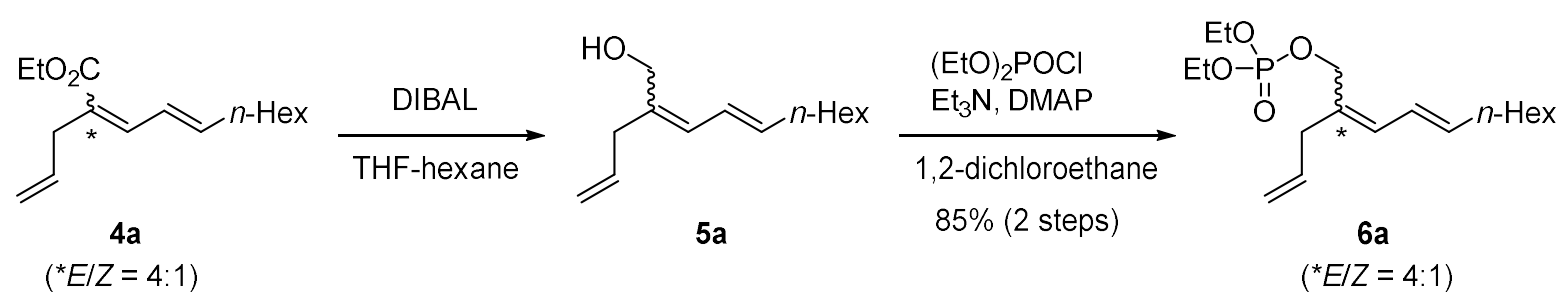

To a solution of $4 \mathbf{a}(E / Z=4: 1,1.21 \mathrm{~g}, 4.82 \mathrm{mmol})$ in THF $(24.1 \mathrm{~mL})$ was added DIBAL $(1.03 \mathrm{~mol} / \mathrm{L}$ in hexane, $14.0 \mathrm{~mL}, 14.4 \mathrm{mmol})$ at $-78^{\circ} \mathrm{C}$. The reaction mixture was then warmed up to $-30{ }^{\circ} \mathrm{C}$ and stirred for $30 \mathrm{~min}$. The reaction was quenched with saturated aqueous Rochelle salt (potassium sodium (+)-tartrate) solution $(40 \mathrm{~mL})$, and the biphasic mixture was stirred vigorously overnight. Two layers were separated, and the aqueous layer was extracted with EtOAc $(30 \mathrm{~mL} \times 3)$. The combined organic layer was dried over $\mathrm{MgSO}_{4}$, filtered, and concentrated under reduced pressure to afford a crude alcohol $\mathbf{5 a}$ (1.02 g, > 99\% crude yield).

To a solution of the crude $\mathbf{5 a}(208 \mathrm{mg}$, ca. $1.00 \mathrm{mmol})$ in 1,2-dichloroethane $(5.00 \mathrm{~mL})$ were added 4-dimethylaminopyridine (DMAP) $(24.7 \mathrm{mg}, 202 \mu \mathrm{mol}), \mathrm{Et}_{3} \mathrm{~N}$ (418 $\mu \mathrm{L}, 3.00 \mathrm{mmol}$ ), and $(\mathrm{EtO})_{2} \mathrm{POCl}(288 \mu \mathrm{L}, 2.00 \mathrm{mmol})$ successively at room temperature. The reaction mixture was then warmed up to $50{ }^{\circ} \mathrm{C}$ and stirred for $2.5 \mathrm{~h}$. The reaction was quenched with saturated aqueous $\mathrm{NaHCO}_{3}$ solution $(5 \mathrm{~mL})$. Two layers were separated, and the aqueous layer was extracted with EtOAc $(5 \mathrm{~mL} \times 3)$. The combined organic layer was dried over $\mathrm{MgSO}_{4}$, filtered, and concentrated under reduced pressure. The resulting residue was purified by flash column chromatography $\left(\mathrm{SiO}_{2}\right.$, hexane:EtOAc:Et $\left.{ }_{3} \mathrm{~N}=3: 1: 0.2\right)$ to afford $\mathbf{6 a}(287 \mathrm{mg}, 834 \mu \mathrm{mol}$, ca. $85 \%$ yield $)$ as a yellow oil.

6a: ${ }^{1} \mathrm{H}$ NMR (500 MHz, $\left.\mathrm{CDCl}_{3}\right) \delta$ 6.34-6.29 (m, 0.2H), 6.25-6.20 (m, 0.8H), $6.15(\mathrm{~d}, J=11.5 \mathrm{~Hz}$, $0.8 \mathrm{H}), 6.01(\mathrm{~d}, J=10.9 \mathrm{~Hz}, 0.2 \mathrm{H}), 5.83-5.71(\mathrm{~m}, 2 \mathrm{H}), 5.10-5.02(\mathrm{~m}, 2 \mathrm{H}), 4.64(\mathrm{~d}, J=6.9 \mathrm{~Hz}, 0.2$ $\times 2 \mathrm{H}), 4.46(\mathrm{~d}, J=6.9 \mathrm{~Hz}, 0.8 \times 2 \mathrm{H}), 4.13-4.07(\mathrm{~m}, 4 \mathrm{H}), 2.97(\mathrm{~d}, J=6.3 \mathrm{~Hz}, 0.8 \times 2 \mathrm{H}), 2.91(\mathrm{~d}, J$ $=6.3 \mathrm{~Hz}, 0.2 \times 2 \mathrm{H}), 2.13-2.09(\mathrm{~m}, 2 \mathrm{H}), 1.40-1.27(\mathrm{~m}, 14 \mathrm{H}), 0.88(\mathrm{t}, J=6.6 \mathrm{~Hz}, 3 \mathrm{H}) ;{ }^{13} \mathrm{C} \mathrm{NMR}$ $\left(126 \mathrm{MHz}, \mathrm{CDCl}_{3}\right) \delta 137.8,137.3,135.5,134.9,131.3(\mathrm{~d}, J=7.2 \mathrm{~Hz}), 131.1,131.0(\mathrm{~d}, J=7.2 \mathrm{~Hz})$, $129.9,125.0,124.9,116.8,116.0,70.8(\mathrm{~d}, J=6.0 \mathrm{~Hz}), 64.3(\mathrm{~d}, J=4.8 \mathrm{~Hz}), 63.6(\mathrm{~d}, J=6.0 \mathrm{~Hz}, 2 \mathrm{C})$, $63.6(\mathrm{~d}, J=6.0 \mathrm{~Hz}, 2 \mathrm{C}), 38.9,32.9,32.8,32.3,31.6,31.6,29.2,29.1,28.8,28.8,22.5,16.1,16.0$, $14.0(2 \mathrm{C}+2 \mathrm{C})\left(1\right.$ peak missing); FT-IR (ATR) $v$ 2957, 2926, 2855, 1263, 1028, $965 \mathrm{~cm}^{-1}$; HRMS (FD) calcd for $\mathrm{C}_{18} \mathrm{H}_{33} \mathrm{O}_{4} \mathrm{P}\left(\mathrm{M}^{+}\right)$: 344.2116, found: 344.2124 . 


\section{General procedure for preparation of cyclization precursors}

\section{Procedure C: Ester reduction / Carbamoylation}

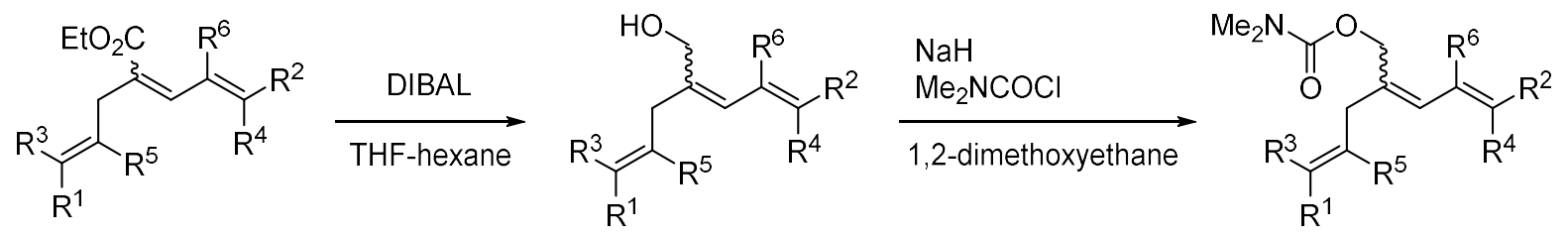

To a solution of $\alpha, \beta$-unsaturated ester in THF $(0.2 \mathrm{~mol} / \mathrm{L})$ was added DIBAL $(1.03 \mathrm{~mol} / \mathrm{L}$ in hexane, 3.0 equiv.) at $-78{ }^{\circ} \mathrm{C}$. The reaction mixture was then warmed up to $-30{ }^{\circ} \mathrm{C}$ and stirred until the full consumption of the substrate was observed (time 1, 30-45 min). The reaction was quenched with saturated aqueous Rochelle salt (potassium sodium (+)-tartrate) solution, and the biphasic mixture was stirred vigorously for over $3 \mathrm{~h}$. The two layers were separated, and the aqueous layer was extracted with EtOAc (3 times). The combined organic layer was dried over $\mathrm{MgSO}_{4}$, filtered, and concentrated under reduced pressure to afford a crude product.

$\mathrm{NaH}$ (60\% dispersion in mineral oil, 2.0 or 3.0 equiv.) was washed with hexane and dissolved with 1,2-dimethoxyethane $(0.4 \mathrm{~mol} / \mathrm{L})$ at $0{ }^{\circ} \mathrm{C}$. To this mixture was added the crude alcohol in 1,2dimethoxyethane and $\mathrm{Me}_{2} \mathrm{NCOCl}$ (2.0 equiv.) successively at the same temperature. The reaction mixture was then warmed up to room temperature and stirred overnight (time 2). The reaction was quenched with saturated aqueous $\mathrm{NH}_{4} \mathrm{Cl}$ solution, and the mixture was extracted with EtOAc (5 times). The combined organic layer was dried over $\mathrm{MgSO}_{4}$, filtered, and concentrated under reduced pressure. The resulting residue was purified by flash column chromatography (amino-functionalized $\mathrm{SiO}_{2}$, hexane:EtOAc $\left.=50: 1\right)$ to afford the product.

\section{Trienyl carbamate $7 \mathbf{a}$}

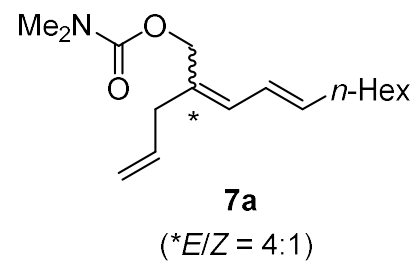

According to procedure $\mathrm{C}$, (time 1: $30 \mathrm{~min}) \mathbf{4 a}(E / Z=4: 1,720 \mathrm{mg}, 2.87$ $\mathrm{mmol})$ and DIBAL $(1.02 \mathrm{~mol} / \mathrm{L}, 8.45 \mathrm{~mL}, 8.62 \mathrm{mmol})$ gave crude alcohol (5a); (time 2: 19 h) crude 5a, $\mathrm{NaH}$ (60\% dispersion in mineral oil, 346 $\mathrm{mg}, 8.64 \mathrm{mmol})$, and $\mathrm{Me}_{2} \mathrm{NCOCl}(528 \mu \mathrm{L}, 5.74 \mathrm{mmol})$ gave $7 \mathbf{a}(E / Z=$ 4:1, $707 \mathrm{mg}, 2.53 \mathrm{mmol}, 88 \%$ ) as a colorless oil.

7a: ${ }^{1} \mathrm{H}$ NMR $\left(500 \mathrm{MHz}, \mathrm{CDCl}_{3}\right) \delta 6.35(\mathrm{dd}, J=14.9,10.9 \mathrm{~Hz}, 0.2 \mathrm{H}), 6.23(\mathrm{dd}, J=14.9,10.9 \mathrm{~Hz}$, $0.8 \mathrm{H}), 6.11(\mathrm{~d}, J=10.9 \mathrm{~Hz}, 0.8 \mathrm{H}), 5.99$ (d, $J=10.9 \mathrm{~Hz}, 0.2 \mathrm{H}), 5.85-5.69$ (m, 2H), 5.08-5.00 (m, $2 \mathrm{H}), 4.70(\mathrm{~s}, 0.2 \times 2 \mathrm{H}), 4.53(\mathrm{~s}, 0.8 \times 2 \mathrm{H}), 2.95-2.87(\mathrm{~m}, 8 \mathrm{H}), 2.13-2.07(\mathrm{~m}, 2 \mathrm{H}), 1.41-1.35(\mathrm{~m}$, 
2H), 1.32-1.25 (m, 6H), $0.88(\mathrm{t}, J=6.9 \mathrm{~Hz}, 3 \mathrm{H}) ;{ }^{13} \mathrm{C} \mathrm{NMR}\left(126 \mathrm{MHz}, \mathrm{CDCl}_{3}\right) \delta 156.6,156.4,136.9$, $136.5,135.8,135.2,132.1,131.8,130.3,129.0,125.2,116.4,115.7,115.7,69.0,62.8,39.5,36.4$ ($\left.\mathrm{NMe}_{2}\right), 35.8$ (-NMe $)$, 32.9, 32.9, 31.7, 29.2, 29.2, 28.9, 28.8, 22.6, 14.1 (overlapped) (3 peaks missing); FT-IR (ATR) v 3026, 2925, 2855, 1706, 1397, $1179 \mathrm{~cm}^{-1}$; HRMS (FD) calcd for $\mathrm{C}_{17} \mathrm{H}_{29} \mathrm{NO}_{2}\left(\mathrm{M}^{+}\right): 279.2198$, found: 279.2207 .

\section{Trienyl carbamate $7 \mathrm{~b}$}

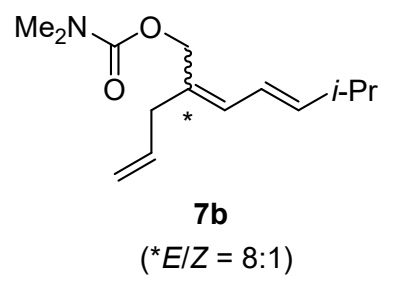

According to procedure $\mathrm{C}$, (time 1: $30 \mathrm{~min}) \mathbf{4 b}(E / Z=8: 1,871 \mathrm{mg}, 4.18$ $\mathrm{mmol})$ and DIBAL (1.03 mol/L, $12.2 \mathrm{~mL}, 12.5 \mathrm{mmol})$ gave crude alcohol (708 mg, > 99\% crude yield); (time 2: $19 \mathrm{~h}$ ) crude alcohol (167 mg, ca. $1.00 \mathrm{mmol}), \mathrm{NaH}$ (60\% dispersion in mineral oil, $80.2 \mathrm{mg}, 2.01 \mathrm{mmol})$, and $\mathrm{Me}_{2} \mathrm{NCOCl}(184 \mu \mathrm{L}, 2.00 \mathrm{mmol})$ gave $7 \mathbf{b}(E / Z=8: 1,222 \mathrm{mg}, 935 \mu \mathrm{mol}$, ca. $92 \%)$ as a colorless oil.

7b: ${ }^{1} \mathrm{H}$ NMR $\left(500 \mathrm{MHz}, \mathrm{CDCl}_{3}\right) \delta 6.32(\mathrm{dd}, J=14.6,11.1 \mathrm{~Hz}, 0.11 \mathrm{H}), 6.20(\mathrm{dd}, J=14.6,11.1 \mathrm{~Hz}$, $0.89 \mathrm{H}), 6.10(\mathrm{~d}, J=11.1 \mathrm{~Hz}, 0.89 \mathrm{H}), 5.98(\mathrm{~d}, J=11.1 \mathrm{~Hz}, 0.11 \mathrm{H}), 5.84-5.66(\mathrm{~m}, 2 \mathrm{H}), 5.09-5.01$ $(\mathrm{m}, 2 \mathrm{H}), 4.71(\mathrm{~s}, 0.11 \times 2 \mathrm{H}), 4.53(\mathrm{~s}, 0.89 \times 2 \mathrm{H}), 2.96-2.91(\mathrm{~m}, 8 \mathrm{H}), 2.40-2.33(\mathrm{~m}, 1 \mathrm{H}), 1.02-0.98$ $(\mathrm{m}, 6 \mathrm{H}) ;{ }^{13} \mathrm{C} \mathrm{NMR}$ for $(E)-7 \mathbf{b}\left(126 \mathrm{MHz}, \mathrm{CDCl}_{3}\right) \delta 156.4,143.8,135.2,132.0,129.1,122.3,115.7$, 68.9, $36.4\left(-\mathrm{NMe}_{2}\right), 35.8\left(-\mathrm{NMe}_{2}\right), 33.0,31.4,22.3$ (2C); FT-IR (ATR) v 2958, 2930, 2869, 1703, 1397, $1176 \mathrm{~cm}^{-1}$; HRMS (FD) calcd for $\mathrm{C}_{14} \mathrm{H}_{23} \mathrm{NO}_{2}\left(\mathrm{M}^{+}\right): 237.1729$, found: 237.1726 .

\section{Trienyl carbamate 7c}

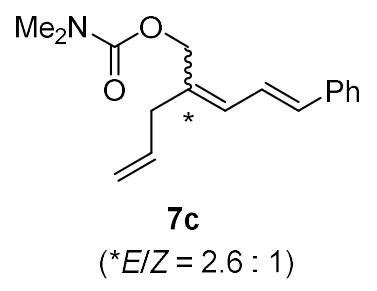

According to procedure $\mathrm{C}$, (time 1: $30 \mathrm{~min}) \mathbf{4 c}(E / Z=2.7: 1,738 \mathrm{mg}, 3.05$ mmol) and DIBAL (1.03 mol/L, $8.87 \mathrm{~mL}, 9.14 \mathrm{mmol})$ gave crude alcohol (635 mg, > 99\% crude yield); (time 2: 19 h) crude alcohol (40.1 mg, ca. 200 $\mu \mathrm{mol}), \mathrm{NaH}(60 \%$ dispersion in mineral oil, $16.1 \mathrm{mg}, 403 \mu \mathrm{mol})$, and $\mathrm{Me}_{2} \mathrm{NCOCl}(36.8 \mu \mathrm{L}, 400 \mu \mathrm{mol})$ gave $7 \mathrm{c}(E / Z=2.6: 1,46.4 \mathrm{mg}, 171 \mu \mathrm{mol}$, ca. $89 \%$ ) as a colorless oil.

7c: ${ }^{1} \mathrm{H}$ NMR (500 MHz, $\left.\mathrm{CDCl}_{3}\right) \delta 7.41(\mathrm{~d}, J=7.4 \mathrm{~Hz}, 2 \mathrm{H}), 7.33-7.30(\mathrm{~m}, 2 \mathrm{H}), 7.26-7.21(\mathrm{~m}, 1 \mathrm{H})$, $7.12(\mathrm{dd}, J=15.2,11.4 \mathrm{~Hz}, 0.28 \mathrm{H}), 6.99(\mathrm{dd}, J=15.5,10.9 \mathrm{~Hz}, 0.72 \mathrm{H}), 6.61-6.54(\mathrm{~m}, 1 \mathrm{H}), 6.32(\mathrm{~d}$, $J=10.9 \mathrm{~Hz}, 0.72 \mathrm{H}), 6.20(\mathrm{~d}, J=11.4 \mathrm{~Hz}, 0.28 \mathrm{H}), 5.88-5.79(\mathrm{~m}, 1 \mathrm{H}), 5.14-5.05(\mathrm{~m}, 2 \mathrm{H}), 4.82(\mathrm{~s}$, $0.28 \times 2 \mathrm{H}), 4.61(\mathrm{~s}, 0.72 \times 2 \mathrm{H}), 3.07(\mathrm{~d}, J=6.3 \mathrm{~Hz}, 0.72 \times 2 \mathrm{H}), 2.97-2.91(\mathrm{~m}, 6 \mathrm{H}+0.28 \times 2 \mathrm{H}) ;{ }^{13} \mathrm{C}$ NMR $\left(126 \mathrm{MHz}, \mathrm{CDCl}_{3}\right) \delta 156.5,156.4,137.3,137.3,135.5,135.4,135.1,135.1,133.7,133.3$, 130.2, $128.6(2 \mathrm{C}), 128.6(2 \mathrm{C}), 127.6,127.6,126.4(2 \mathrm{C}), 126.4(2 \mathrm{C}), 124.0,123.9,116.8,116.1,68.7$, 62.8, 39.9, 36.5 (-NMe 2$), 35.9\left(-\mathrm{NMe}_{2}\right), 33.2$ (1 peak missing); FT-IR (ATR) v 3029, 2932, 1698, 
1397, $1176 \mathrm{~cm}^{-1}$; HRMS (FD) calcd for $\mathrm{C}_{17} \mathrm{H}_{21} \mathrm{NO}_{2}\left(\mathrm{M}^{+}\right)$: 271.1572, found: 271.1585 .

\section{Trienyl carbamate 12a}

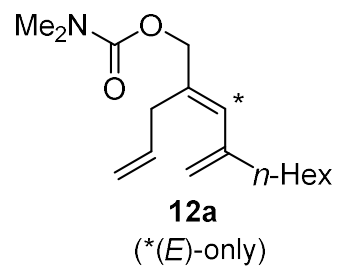

According to procedure C, (time 1: $30 \mathrm{~min}) \mathbf{S 2 1 a}(169 \mathrm{mg}, 676 \mu \mathrm{mol})$ and DIBAL (1.03 mol/L, $1.97 \mathrm{~mL}, 2.03 \mathrm{mmol})$ gave crude alcohol $(144 \mathrm{mg},>$ 99\% crude yield); (time 2: $20 \mathrm{~h}$ ) crude alcohol (41.6 mg, ca. $200 \mu \mathrm{mol}), \mathrm{NaH}$ (60\% dispersion in mineral oil, $15.5 \mathrm{mg}, 388 \mu \mathrm{mol})$, and $\mathrm{Me}_{2} \mathrm{NCOCl}(36.8 \mu \mathrm{L}$, $400 \mu \mathrm{mol})$ gave 12a (48.3 mg, $173 \mu \mathrm{mol}$, ca. $89 \%)$ as a colorless oil.

12a: ${ }^{1} \mathrm{H}$ NMR $\left(500 \mathrm{MHz}, \mathrm{CDCl}_{3}\right) \delta 5.96(\mathrm{~s}, 1 \mathrm{H}), 5.84-5.76(\mathrm{~m}, 1 \mathrm{H}), 5.08-5.03(\mathrm{~m}, 2 \mathrm{H}), 4.97(\mathrm{~s}$, $1 \mathrm{H}), 4.90(\mathrm{~s}, 1 \mathrm{H}), 4.55(\mathrm{~s}, 2 \mathrm{H}), 3.01(\mathrm{~d}, J=6.3 \mathrm{~Hz}, 2 \mathrm{H}), 2.93(\mathrm{~s}, 6 \mathrm{H}), 2.08$ (t, $J=7.4 \mathrm{~Hz}, 2 \mathrm{H}), 1.41-$ $1.35(\mathrm{~m}, 2 \mathrm{H}), 1.31-1.26(\mathrm{~m}, 6 \mathrm{H}), 0.88(\mathrm{t}, J=6.9 \mathrm{~Hz}, 3 \mathrm{H}) ;{ }^{13} \mathrm{C} \mathrm{NMR}\left(126 \mathrm{MHz}, \mathrm{CDCl}_{3}\right) \delta 156.4$, 145.2, 135.9, 134.4, 129.9, 116.0, 113.1, 68.5, 37.2, $36.5\left(-\mathrm{NMe}_{2}\right), 35.8\left(-\mathrm{NMe}_{2}\right), 33.6,31.7,28.8$, 28.1, 22.6, 14.1; FT-IR (ATR) v 3080, 2927, 2856, 1708, 1397, $1181 \mathrm{~cm}^{-1}$; HRMS (FI) calcd for $\mathrm{C}_{17} \mathrm{H}_{29} \mathrm{NO}_{2}\left(\mathrm{M}^{+}\right): 279.2198$, found: 279.2199 .

\section{Trienyl carbamate 12b}

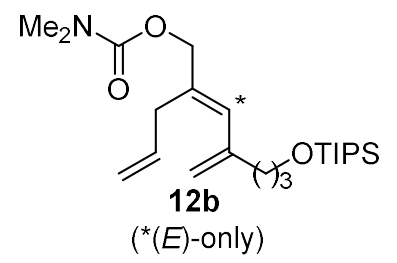

According to procedure C, (time 1: $30 \mathrm{~min}) \mathbf{S 2 1 b}(80.6 \mathrm{mg}, 212 \mu \mathrm{mol})$ and DIBAL $(1.03 \mathrm{~mol} / \mathrm{L}, 617 \mu \mathrm{L}, 635 \mu \mathrm{mol})$ gave crude alcohol; (time 2: $17 \mathrm{~h})$ crude alcohol, $\mathrm{NaH}(60 \%$ dispersion in mineral oil, $16.8 \mathrm{mg}, 420$ $\mu \mathrm{mol})$, and $\mathrm{Me}_{2} \mathrm{NCOCl}(39.0 \mu \mathrm{L}, 424 \mu \mathrm{mol})$ gave $\mathbf{1 2 b}(84.0 \mathrm{mg}, 205 \mu \mathrm{mol}$, $97 \%$ for 2 steps) as a colorless oil.

12b: ${ }^{1} \mathrm{H}$ NMR (500 MHz, $\left.\mathrm{CDCl}_{3}\right) \delta 5.97(\mathrm{~s}, 1 \mathrm{H}), 5.84-5.76(\mathrm{~m}, 1 \mathrm{H}), 5.07-5.03(\mathrm{~m}, 2 \mathrm{H}), 5.00(\mathrm{~s}$, $1 \mathrm{H}), 4.92(\mathrm{~s}, 1 \mathrm{H}), 4.55$ (s, 2H), $3.67(\mathrm{t}, J=6.3 \mathrm{~Hz}, 2 \mathrm{H}), 3.00$ (d, $J=6.3 \mathrm{~Hz}, 2 \mathrm{H}), 2.93$ (s, 6H), 2.17 $(\mathrm{t}, J=7.7 \mathrm{~Hz}, 2 \mathrm{H}), 1.67-1.61(\mathrm{~m}, 2 \mathrm{H}), 1.10-1.05(\mathrm{~m}, 21 \mathrm{H}) ;{ }^{13} \mathrm{C} \mathrm{NMR}\left(126 \mathrm{MHz}, \mathrm{CDCl}_{3}\right) \delta 156.3$, 144.6, 135.8, 134.6, 129.7, 116.0, 113.4, 68.5, 62.7, $36.4\left(-\mathrm{NMe}_{2}\right), 35.9\left(-\mathrm{NMe}_{2}\right), 33.7,33.5,31.5$, 18.0 (6C), 11.9 (3C); FT-IR (ATR) v 2941, 2865, 1709, 1463, 1396, 1179, $1103 \mathrm{~cm}^{-1}$; HRMS (FD) calcd for $\mathrm{C}_{23} \mathrm{H}_{43} \mathrm{NO}_{3} \mathrm{Si}\left(\mathrm{M}^{+}\right)$: 409.3012, found: 409.2999. 


\section{Trienyl carbamate 12c}

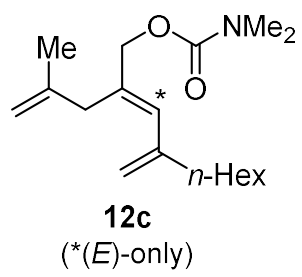

According to procedure C, (time 1: $45 \mathrm{~min})$ S21c $(140 \mathrm{mg}, 529 \mu \mathrm{mol})$ and DIBAL (1.03 mol/L, $1.54 \mathrm{~mL}, 1.59 \mathrm{mmol})$ gave crude alcohol $(117 \mathrm{mg},>99 \%$ crude yield); (time 2: $17 \mathrm{~h}$ ) crude alcohol (66.3 mg, ca. $300 \mu \mathrm{mol}), \mathrm{NaH}(60 \%$ dispersion in mineral oil, $23.7 \mathrm{mg}, 593 \mu \mathrm{mol})$, and $\mathrm{Me}_{2} \mathrm{NCOCl}(55.1 \mu \mathrm{L}, 600$ $\mu \mathrm{mol})$ gave 12c (78.9 mg, $269 \mu \mathrm{mol}$, ca. 90\%) as a colorless oil.

12c: ${ }^{1} \mathrm{H}$ NMR $\left(500 \mathrm{MHz}, \mathrm{CDCl}_{3}\right) \delta 6.02(\mathrm{~s}, 1 \mathrm{H}), 4.94(\mathrm{~s}, 1 \mathrm{H}), 4.91(\mathrm{~s}, 1 \mathrm{H}), 4.79(\mathrm{~s}, 1 \mathrm{H}), 4.69(\mathrm{~s}$, $1 \mathrm{H}), 4.52(\mathrm{~s}, 2 \mathrm{H}), 2.94-2.92(\mathrm{~m}, 8 \mathrm{H}), 2.07$ (t, $J=7.4 \mathrm{~Hz}, 2 \mathrm{H}), 1.71(\mathrm{~s}, 3 \mathrm{H}), 1.41-1.36(\mathrm{~m}, 2 \mathrm{H}), 1.31-$ $1.25(\mathrm{~m}, 6 \mathrm{H}), 0.88(\mathrm{t}, J=6.6 \mathrm{~Hz}, 3 \mathrm{H}) ;{ }^{13} \mathrm{C} \mathrm{NMR}\left(126 \mathrm{MHz}, \mathrm{CDCl}_{3}\right) \delta 156.4,145.1,143.0,134.2$, 130.4, 112.7, 111.4, 68.7, 37.4, 37.3, $36.4\left(-\mathrm{NMe}_{2}\right), 35.9\left(-\mathrm{NMe}_{2}\right), 31.7,28.9,28.1$, 22.9, 22.6, 14.1; FT-IR (ATR) v 3078, 2927, 2856, 1707, 1397, $1178 \mathrm{~cm}^{-1}$; HRMS (FD) calcd for $\mathrm{C}_{18} \mathrm{H}_{31} \mathrm{NO}_{2}\left(\mathrm{M}^{+}\right)$: 293.2355, found: 293.2357 .

\section{Trienyl carbamate 12d}

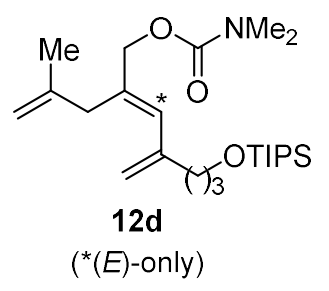

According to procedure C, (time 1: $30 \mathrm{~min}) \mathbf{S 2 1 d}(59.7 \mathrm{mg}, 151 \mu \mathrm{mol})$ and DIBAL (1.03 mol/L, $441 \mu \mathrm{L}, 454 \mu \mathrm{mol})$ gave crude alcohol; (time 2: $17 \mathrm{~h}$ ) crude alcohol, $\mathrm{NaH}$ (60\% dispersion in mineral oil, $12.3 \mathrm{mg}, 308 \mu \mathrm{mol})$, and $\mathrm{Me}_{2} \mathrm{NCOCl}(27.8 \mu \mathrm{L}, 302 \mu \mathrm{mol})$ gave $12 \mathrm{~d}(62.7 \mathrm{mg}, 148 \mu \mathrm{mol}, 98 \%$ for 2 steps) as a colorless oil.

12d: ${ }^{1} \mathrm{H}$ NMR $\left(500 \mathrm{MHz}, \mathrm{CDCl}_{3}\right) \delta 6.03(\mathrm{~s}, 1 \mathrm{H}), 4.97(\mathrm{~s}, 1 \mathrm{H}), 4.93(\mathrm{~s}, 1 \mathrm{H}), 4.79(\mathrm{~s}, 1 \mathrm{H}), 4.69$ (s, 1H), $4.52(\mathrm{~s}, 2 \mathrm{H}), 3.67$ (t, $J=6.3 \mathrm{~Hz}, 2 \mathrm{H}), 2.93-2.90(\mathrm{~m}, 8 \mathrm{H}), 2.17$ (t, $J=7.7 \mathrm{~Hz}, 2 \mathrm{H}), 1.71(\mathrm{~s}, 3 \mathrm{H})$, $1.67-1.62(\mathrm{~m}, 2 \mathrm{H}), 1.10-1.04(\mathrm{~m}, 21 \mathrm{H}) ;{ }^{13} \mathrm{C} \mathrm{NMR}\left(126 \mathrm{MHz}, \mathrm{CDCl}_{3}\right) \delta 156.4,144.6,143.0,134.4$, 130.1, 113.0, 111.4, 68.6, 62.8, 37.4, $36.4\left(-\mathrm{NMe}_{2}\right), 35.8$ (-NMe $)$, 33.6, 31.5, 22.9, 18.0 (6C), 12.0 (3C); FT-IR (ATR) v 2941, 2865, 1709, 1462, 1396, 1179, 1103, $882 \mathrm{~cm}^{-1}$; HRMS (FD) calcd for $\mathrm{C}_{24} \mathrm{H}_{45} \mathrm{NO}_{3} \mathrm{Si}\left(\mathrm{M}^{+}\right): 423.3169$, found: 423.3186 .

\section{Trienyl carbamate 14}

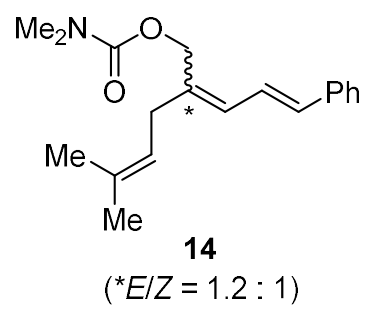

According to procedure C, (time 1: $30 \mathrm{~min}) \mathbf{S 2 7}(E / Z=1.2: 1,243 \mathrm{mg}$, $900 \mu \mathrm{mol})$ and DIBAL $(1.03 \mathrm{~mol} / \mathrm{L}, 2.62 \mathrm{~mL}, 2.70 \mathrm{mmol})$ gave crude alcohol (209 mg, > 99\% crude yield); (time 2: $1.5 \mathrm{~d}$ ) crude alcohol (45.7 mg, ca. $200 \mu \mathrm{mol}), \mathrm{NaH}(60 \%$ dispersion in mineral oil, $16.6 \mathrm{mg}, 415 \mu \mathrm{mol})$, and $\mathrm{Me}_{2} \mathrm{NCOCl}(36.8 \mu \mathrm{L}, 400 \mu \mathrm{mol})$ gave $14(E / Z=1.2: 1,39.1 \mathrm{mg}, 131$ $\mu \mathrm{mol}$, ca. $66 \%$ ) as a yellow oil. 
14: ${ }^{1} \mathrm{H}$ NMR (500 MHz, $\left.\mathrm{CDCl}_{3}\right) \delta 7.41-7.40(\mathrm{~m}, 2 \mathrm{H}), 7.33-7.20(\mathrm{~m}, 3 \mathrm{H}), 7.12(\mathrm{dd}, J=15.5,11.5 \mathrm{~Hz}$, $0.45 \mathrm{H}), 7.04(\mathrm{dd}, J=15.5,11.2 \mathrm{~Hz}, 0.55 \mathrm{H}), 6.59-6.53(\mathrm{~m}, 1 \mathrm{H}), 6.23(\mathrm{~d}, J=11.2 \mathrm{~Hz}, 0.55 \mathrm{H}), 6.17$ (d, $J=11.5 \mathrm{~Hz}, 0.45 \mathrm{H}), 5.19(\mathrm{t}, J=7.4 \mathrm{~Hz}, 0.45 \mathrm{H}), 5.11(\mathrm{t}, J=7.4 \mathrm{~Hz}, 0.55 \mathrm{H}), 4.79(\mathrm{~s}, 0.45 \times 2 \mathrm{H}), 4.58$ $(\mathrm{s}, 0.55 \times 2 \mathrm{H}), 3.02(\mathrm{~d}, J=7.4 \mathrm{~Hz}, 0.55 \times 2 \mathrm{H}), 2.94-2.90(\mathrm{~m}, 6 \mathrm{H}+0.45 \times 2 \mathrm{H}), 1.75(\mathrm{~s}, 0.45 \times 3 \mathrm{H})$, $1.71(\mathrm{~s}, 0.55 \times 6 \mathrm{H}), 1.65(\mathrm{~s}, 0.45 \times 3 \mathrm{H}) ;{ }^{13} \mathrm{C} \mathrm{NMR}\left(126 \mathrm{MHz}, \mathrm{CDCl}_{3}\right) \delta 156.6,156.4,137.4,137.4$, $137.3,136.7,133.8,133.2,132.9,132.8,129.2,128.6(2 \mathrm{C}), 128.5$ (2C), 127.5, 127.4, 127.3, 126.4 (2C), 126.3 (2C), 124.1, 124.1, 121.3, 121.0, 68.7, 63.0, $36.4\left(-\mathrm{NMe}_{2}\right), 35.8\left(-\mathrm{NMe}_{2}\right), 34.1,27.9,25.8$, 25.7, 17.8, 17.7; FT-IR (ATR) v 3027, 2966, 2926, 1700, 1397, $1176 \mathrm{~cm}^{-1}$; HRMS (FI) calcd for $\mathrm{C}_{19} \mathrm{H}_{25} \mathrm{NO}_{2}\left(\mathrm{M}^{+}\right): 299.1885$, found: 299.1875 .

\section{Trienyl carbamate 16}

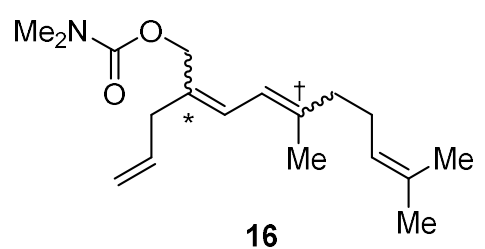

$\left({ }^{*} E / Z=10: 1,{ }^{\dagger} E / Z=\right.$ ca. $\left.1: 1\right)$

According to procedure C, (time 1: $30 \mathrm{~min}) \mathbf{S 2 8}(2 E / 2 Z=13: 1$, $4 E / 4 Z=1: 1,184 \mathrm{mg}, 701 \mu \mathrm{mol})$ and DIBAL $(1.03 \mathrm{~mol} / \mathrm{L}, 2.04 \mathrm{~mL}$, $2.10 \mathrm{mmol}$ ) gave crude alcohol; (time 2: $18 \mathrm{~h}$ ) crude alcohol, $\mathrm{NaH}$ (60\% dispersion in mineral oil, $56.4 \mathrm{mg}, 1.41 \mathrm{mmol}$ ), and $\mathrm{Me}_{2} \mathrm{NCOCl}$ $(129 \mu \mathrm{L}, 1.40 \mathrm{mmol})$ gave $\mathbf{1 6}(2 E / 2 Z=13: 1,4 E / 4 Z=$ ca. $1: 1,136 \mathrm{mg}$, $467 \mu \mathrm{mol}, 67 \%$ for 2 steps) as a yellow oil.

16: ${ }^{1} \mathrm{H}$ NMR for $(2 E, 4 E)-16$ and (2E,4Z)-16 $\left(500 \mathrm{MHz}, \mathrm{CDCl}_{3}\right) \delta 6.36-6.33(\mathrm{~m}, 1 \mathrm{H}), 6.03(\mathrm{~s}, 0.53 \mathrm{H})$, $6.01(\mathrm{~s}, 0.47 \mathrm{H}), 5.82-5.74(\mathrm{~m}, 1 \mathrm{H}), 5.12-5.00(\mathrm{~m}, 3 \mathrm{H}), 4.56-4.55(\mathrm{~d}, J=8.0 \mathrm{~Hz}, 2 \mathrm{H}), 2.96-2.92(\mathrm{~m}$, $8 \mathrm{H}), 2.20-2.06(\mathrm{~m}, 4 \mathrm{H}), 1.82(\mathrm{~s}, 0.47 \times 3 \mathrm{H}), 1.77(\mathrm{~s}, 0.53 \times 3 \mathrm{H}), 1.68(\mathrm{~s}, 0.53 \times 6 \mathrm{H}), 1.61(\mathrm{~s}, 0.47$ $\times 6 \mathrm{H}) ;{ }^{13} \mathrm{C}$ NMR for $(2 E, 4 E)-16$ and $(2 E, 4 Z)-16\left(126 \mathrm{MHz}, \mathrm{CDCl}_{3}\right) \delta 156.5$ (overlapped), 141.0, $140.8,135.3,135.3,131.9,131.7,131.7,131.4,125.1,124.8,123.9,123.8,120.4,119.7,115.6$, 115.6, 69.5, 69.5, 40.3, $36.4\left(-\mathrm{NMe}_{2}\right), 35.8\left(-\mathrm{NMe}_{2}\right), 32.9,32.8,32.4,26.7,26.5,25.6,24.3,17.7$, 17.6, 16.7 (1 peak missing); FT-IR (ATR) v 2965, 2923, 2857, 1703, 1396, $1177 \mathrm{~cm}^{-1}$; HRMS (FD) calcd for $\mathrm{C}_{18} \mathrm{H}_{29} \mathrm{NO}_{2}\left(\mathrm{M}^{+}\right)$: 291.2198, found: 291.2206 .

\section{Trienyl carbamate 18a}

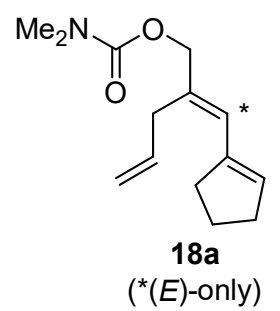

According to procedure C, (time 1: $30 \mathrm{~min})$ S22a (179 $\mathrm{mg}, 866 \mu \mathrm{mol})$ and DIBAL (1.02 mol/L, $2.55 \mathrm{~mL}, 2.60 \mathrm{mmol}$ ) gave crude alcohol; (time 2: $14 \mathrm{~h}$ ) crude alcohol, $\mathrm{NaH}(60 \%$ dispersion in mineral oil, $105 \mathrm{mg}, 2.63 \mathrm{mmol})$, and $\mathrm{Me}_{2} \mathrm{NCOCl}(159 \mu \mathrm{L}, 1.73 \mathrm{mmol})$ gave 18a (182 mg, $771 \mu \mathrm{mol}, 89 \%$ for 2 steps) as a colorless oil.

18a: ${ }^{1} \mathrm{H}$ NMR $\left(500 \mathrm{MHz}, \mathrm{CDCl}_{3}\right) \delta 6.21(\mathrm{~s}, 1 \mathrm{H}), 5.85-5.78(\mathrm{~m}, 1 \mathrm{H}), 5.76(\mathrm{~s}, 1 \mathrm{H}), 5.07-5.02(\mathrm{~m}$, 
2H), $4.54(\mathrm{~s}, 2 \mathrm{H}), 3.04(\mathrm{~d}, J=5.7 \mathrm{~Hz}, 2 \mathrm{H}), 2.92(\mathrm{~s}, 6 \mathrm{H}), 2.56-2.53(\mathrm{~m}, 2 \mathrm{H}), 2.36(\mathrm{t}, J=6.0 \mathrm{~Hz}, 2 \mathrm{H})$, $1.93-1.87(\mathrm{~m}, 2 \mathrm{H}) ;{ }^{13} \mathrm{C} \mathrm{NMR}\left(126 \mathrm{MHz}, \mathrm{CDCl}_{3}\right) \delta 156.5,140.5,135.8,132.7,132.6,126.1,115.7$, 69.6, $36.4\left(-\mathrm{NMe}_{2}\right), 35.9\left(-\mathrm{NMe}_{2}\right), 34.6,33.5,32.3,23.9$; FT-IR (ATR) v 3078, 3037, 2892, 2842 , 1702, 1397, $1177 \mathrm{~cm}^{-1}$; HRMS (FD) calcd for $\mathrm{C}_{14} \mathrm{H}_{21} \mathrm{NO}_{2}\left(\mathrm{M}^{+}\right)$: 235.1572, found: 235.1581 .

\section{Trienyl carbamate $18 b$}

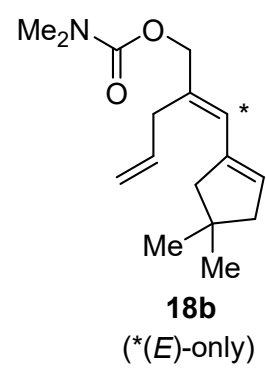

According to procedure C, (time 1: $30 \mathrm{~min})$ S22b (109 $\mathrm{mg}, 467 \mu \mathrm{mol})$ and DIBAL (1.02 mol/L, $1.37 \mathrm{~mL}, 1.40 \mathrm{mmol}$ ) gave crude alcohol; (time 2: $17 \mathrm{~h}$ ) crude alcohol, $\mathrm{NaH}(60 \%$ dispersion in mineral oil, $56.0 \mathrm{mg}, 1.40 \mathrm{mmol})$, and $\mathrm{Me}_{2} \mathrm{NCOCl}(85.8 \mu \mathrm{L}, 934 \mu \mathrm{mol})$ gave $\mathbf{1 8 b}(115 \mathrm{mg}, 438 \mu \mathrm{mol}, 94 \%$ for 2 steps $)$ as a colorless oil.

18b: ${ }^{1} \mathrm{H}$ NMR $\left(500 \mathrm{MHz}, \mathrm{CDCl}_{3}\right) \delta 6.15(\mathrm{~s}, 1 \mathrm{H}), 5.85-5.77(\mathrm{~m}, 1 \mathrm{H}), 5.63(\mathrm{~s}, 1 \mathrm{H}), 5.07-5.02(\mathrm{~m}$, 2H), 4.53 (s, 2H), 3.01 (d, $J=5.7 \mathrm{~Hz}, 2 \mathrm{H}), 2.92(\mathrm{~s}, 6 \mathrm{H}), 2.37$ (d, $J=1.7 \mathrm{~Hz}, 2 \mathrm{H}), 2.18$ (s, 2H), 1.09 $(\mathrm{s}, 6 \mathrm{H}) ;{ }^{13} \mathrm{C} \mathrm{NMR}\left(126 \mathrm{MHz}, \mathrm{CDCl}_{3}\right) \delta 156.5,139.2,135.9,132.4,131.5,126.5,115.8,69.6,49.8$, 47.3, 39.0, $36.4\left(-\mathrm{NMe}_{2}\right), 35.9$ (-NMe $)$, 33.5, 29.7 (2C); FT-IR (ATR) v 3078, 3035, 2952, 2866, 2837, 1703, 1397, $1177 \mathrm{~cm}^{-1}$; HRMS (FD) calcd for $\mathrm{C}_{16} \mathrm{H}_{25} \mathrm{NO}_{2}\left(\mathrm{M}^{+}\right)$: 263.1885, found: 263.1893.

\section{Trienyl carbamate 20a}

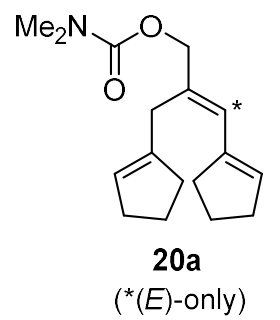

According to procedure C, (time 1: $40 \mathrm{~min}$ ) S23a (398 $\mathrm{mg}, 1.62 \mathrm{mmol}$ ) and DIBAL (1.02 mol/L, $4.75 \mathrm{~mL}, 4.85 \mathrm{mmol})$ gave crude alcohol; (time 2: $15 \mathrm{~h}$ ) crude alcohol, $\mathrm{NaH}$ (60\% dispersion in mineral oil, $196 \mathrm{mg}, 4.91 \mathrm{mmol})$, and $\mathrm{Me}_{2} \mathrm{NCOCl}(297 \mu \mathrm{L}, 3.23 \mathrm{mmol}$ ) gave 20a (434 mg, $1.57 \mathrm{mmol}, 97 \%$ for 2 steps) as a yellow oil.

20a: ${ }^{1} \mathrm{H}$ NMR $\left(500 \mathrm{MHz}, \mathrm{CDCl}_{3}\right) \delta 6.22(\mathrm{~s}, 1 \mathrm{H}), 5.73(\mathrm{~s}, 1 \mathrm{H}), 5.33(\mathrm{t}, J=1.7 \mathrm{~Hz}, 1 \mathrm{H}), 4.52(\mathrm{~s}, 2 \mathrm{H})$, $3.00(\mathrm{~s}, 2 \mathrm{H}), 2.93-2.91(\mathrm{~m}, 6 \mathrm{H}), 2.54-2.51(\mathrm{~m}, 2 \mathrm{H}), 2.37-2.34(\mathrm{~m}, 2 \mathrm{H}), 2.31-2.26(\mathrm{~m}, 2 \mathrm{H}), 2.23(\mathrm{t}$, $J=6.6 \mathrm{~Hz}, 2 \mathrm{H}), 1.92-1.83(\mathrm{~m}, 4 \mathrm{H}) ;{ }^{13} \mathrm{C} \mathrm{NMR}\left(126 \mathrm{MHz}, \mathrm{CDCl}_{3}\right) \delta 156.5,141.9,140.7,133.1$, 132.4, 125.8, 125.0, 69.9, $36.4\left(-\mathrm{NMe}_{2}\right), 35.9\left(-\mathrm{NMe}_{2}\right), 35.5,34.5,32.4$, 32.2, 31.3, 23.9, 23.4; FTIR (ATR) v 3038, 2928, 2843, 1703, 1396, $1176 \mathrm{~cm}^{-1}$; HRMS (FD) calcd for $\mathrm{C}_{17} \mathrm{H}_{25} \mathrm{NO}_{2}\left(\mathrm{M}^{+}\right)$: 275.1885, found: 275.1898 . 


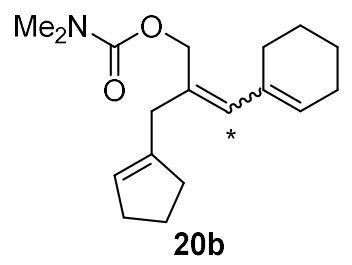

$\left({ }^{*} E / Z=1.6: 1\right)$

According to procedure $\mathrm{C}$, (time 1: $10 \mathrm{~min}$, in $\left.\mathrm{CH}_{2} \mathrm{Cl}_{2}\right) \mathbf{S 2 3 b}(E / Z=1.4: 1$, $69.8 \mathrm{mg}, 268 \mu \mathrm{mol})$ and DIBAL $(1.03 \mathrm{~mol} / \mathrm{L}, 781 \mu \mathrm{L}, 804 \mu \mathrm{mol})$ gave crude alcohol; (time 2: 18 h) crude alcohol, $\mathrm{NaH}(60 \%$ dispersion in mineral oil, $21.4 \mathrm{mg}, 535 \mu \mathrm{mol})$, and $\mathrm{Me}_{2} \mathrm{NCOCl}(49.3 \mu \mathrm{L}, 536 \mu \mathrm{mol})$ gave $20 \mathrm{~b}(E / Z=$ $1.6: 1,70.3 \mathrm{mg}, 243 \mu \mathrm{mol}, 91 \%$ for 2 steps) as a colorless oil.

20b: ${ }^{1} \mathrm{H}$ NMR (500 MHz, $\left.\mathrm{CDCl}_{3}\right) \delta 5.92(\mathrm{~s}, 0.62 \mathrm{H}), 5.80(\mathrm{~s}, 0.38 \mathrm{H}), 5.66(\mathrm{~s}, 0.62 \mathrm{H}), 5.53(\mathrm{~s}, 0.38 \mathrm{H})$, $5.36(\mathrm{~s}, 0.38 \mathrm{H}), 5.32(\mathrm{~s}, 0.62 \mathrm{H}), 4.66(\mathrm{~s}, 0.38 \times 2 \mathrm{H}), 4.48(\mathrm{~s}, 0.62 \times 2 \mathrm{H}), 2.97(\mathrm{~s}, 0.62 \times 2 \mathrm{H}), 2.90-$ $2.88(\mathrm{~m}, 6 \mathrm{H}+0.38 \times 2 \mathrm{H}), 2.28(\mathrm{br}, 2 \mathrm{H}), 2.22-2.17(\mathrm{~m}, 2 \mathrm{H}), 2.08-2.04(\mathrm{~m}, 4 \mathrm{H}), 1.87-1.80(\mathrm{~m}, 2 \mathrm{H})$, $1.63-1.53(\mathrm{~m}, 4 \mathrm{H}) ;{ }^{13} \mathrm{C} \mathrm{NMR}\left(126 \mathrm{MHz}, \mathrm{CDCl}_{3}\right) \delta 156.7,156.5,142.2,142.1,134.5,134.4,133.9$, $132.2,131.9,131.7,127.3,127.2,125.8,125.0,69.8,63.7,37.4,36.4\left(-\mathrm{NMe}_{2}\right), 35.8\left(-\mathrm{NMe}_{2}\right), 35.5$, 34.8, 32.5, 32.4, 31.2 28.9, 28.6, 25.6, 25.5, 23.5, 23.4, 22.8, 22.7, 22.1, 22.0; FT-IR (ATR) v 3035, 2926, 2843, 1703, 1395, $1177 \mathrm{~cm}^{-1}$; HRMS (FI) calcd for $\mathrm{C}_{18} \mathrm{H}_{27} \mathrm{NO}_{2}\left(\mathrm{M}^{+}\right)$: 289.2042, found: 289.2041 .

\section{General procedure for $8 \pi$ electrocyclic reaction}

\section{Procedure D: Cyclization}

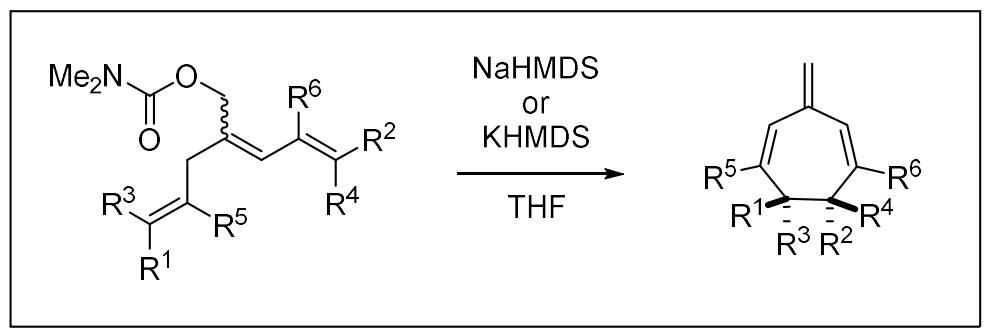

To a solution of trienyl carbamate in THF $(0.1 \mathrm{~mol} / \mathrm{L})$ was added base (NaHMDS or KHMDS in THF, 2.0 equiv.) at $50{ }^{\circ} \mathrm{C}$. The reaction mixture was stirred at the same temperature until no further changes in TLC spots were observed (15 min-2 h). The reaction mixture was then cooled and 3tert-butyl-4-hydroxyanisole (catalytic amount) was added. The reaction was quenched with brine. Two layers were separated, and the aqueous layer was extracted with EtOAc (3 times). The combined organic layer was dried over $\mathrm{MgSO}_{4}$, filtered, and concentrated under reduced pressure. The resulting residue was purified by flash column chromatography $\left[\mathrm{SiO}_{2}\right.$ (neutralized by elution of $N, N$-dimethylaniline), hexane only] to afford the cyclized product. 
NOTE: The cyclized products are unstable and decomposed within a few days in a freezer. Flash column chromatography was performed as quick as possible. Also, $\mathrm{CDCl}_{3}$ ( $\mathrm{NMR}$ solvent) was passed through a column of basic alumina just before using.

\section{Cycloheptatriene 8a}

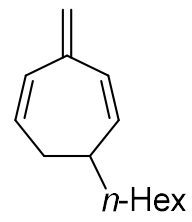

$8 \mathrm{a}$

$\underline{0.1 \mathrm{mmol} \text { scale: }}$ According to procedure D (reaction time: $1 \mathrm{~h}), 7 \mathbf{a}(E / Z=4: 1,37.4 \mathrm{mg}$, $134 \mu \mathrm{mol})$ and NaHMDS (1.10 mol/L in THF, $243 \mu \mathrm{L}, 267 \mu \mathrm{mol})$ gave 8a (16.6 mg, $87.2 \mu \mathrm{mol}, 65 \%$ yield) as a colorless oil.

$1 \mathrm{mmol}$ scale: According to procedure D (reaction time: $1 \mathrm{~h}), 7 \mathbf{a}(E / Z=4: 1,351 \mathrm{mg}$, $1.25 \mathrm{mmol})$ and NaHMDS (1.10 mol/L in THF, $2.27 \mathrm{~mL}, 2.50 \mu \mathrm{mol})$ gave $8 \mathbf{a}(174 \mathrm{mg}$, $915 \mu \mathrm{mol}, 73 \%$ yield) as a colorless oil.

8a: ${ }^{1} \mathrm{H}$ NMR $\left(500 \mathrm{MHz}, \mathrm{CDCl}_{3}\right) \delta 6.08(\mathrm{~d}, J=11.6 \mathrm{~Hz}, 1 \mathrm{H}), 6.03(\mathrm{~d}, J=11.9 \mathrm{~Hz}, 1 \mathrm{H}), 5.77(\mathrm{dt}, J=$ 11.9, 6.0 Hz, 1H), $5.71(\mathrm{dd}, J=11.6,4.9 \mathrm{~Hz}, 1 \mathrm{H}), 5.05$ (s, 1H), 5.04 (s, 1H), 2.41 (br, 1H), 2.35$2.30(\mathrm{~m}, 1 \mathrm{H}), 2.25-2.17(\mathrm{~m}, 1 \mathrm{H}), 1.45-1.27(\mathrm{~m}, 10 \mathrm{H}), 0.88(\mathrm{t}, J=6.9 \mathrm{~Hz}, 3 \mathrm{H}) ;{ }^{13} \mathrm{C} \mathrm{NMR}(126 \mathrm{MHz}$, $\left.\mathrm{CDCl}_{3}\right) \delta 144.0,137.1,130.8,130.1,129.4,120.6,38.6,36.1,33.7,31.8,29.4,27.2$, 22.7, 14.1; FTIR (ATR) v 3086, 3017, 2924, 2853, 1458, 881, $786 \mathrm{~cm}^{-1}$; HRMS (FI) calcd for $\mathrm{C}_{14} \mathrm{H}_{22}\left(\mathrm{M}^{+}\right)$: 190.1722, found: 190.1716 .

\section{Cycloheptatriene 8b}

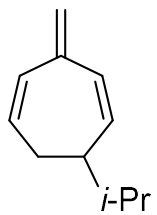

According to procedure D (reaction time: $1 \mathrm{~h}), 7 \mathbf{b}(E / Z=8: 1,48.0 \mathrm{mg}, 202 \mu \mathrm{mol})$ and NaHMDS $(1.10 \mathrm{~mol} / \mathrm{L}$ in THF, $364 \mu \mathrm{L}, 400 \mu \mathrm{mol})$ gave, after purification by flash column chromatography $\left[\mathrm{SiO}_{2}\right.$ (neutralized by elution of $N, N$-dimethylaniline), pentane 8b only], $\mathbf{8 b}$ (22.1 mg, $149 \mu \mathrm{mol}, 74 \%$ yield) as a colorless oil.

8b: ${ }^{1} \mathrm{H}$ NMR (500 MHz, $\left.\mathrm{CDCl}_{3}\right) \delta 6.08(\mathrm{~d}, J=12.0 \mathrm{~Hz}, 2 \mathrm{H}), 5.83-5.76(\mathrm{~m}, 2 \mathrm{H}), 5.05(\mathrm{~d}, J=6.6 \mathrm{~Hz}$, $2 \mathrm{H}), 2.30-2.23(\mathrm{~m}, 3 \mathrm{H}), 1.79-1.72(\mathrm{~m}, 1 \mathrm{H}), 0.91(\mathrm{dd}, J=8.6,6.9 \mathrm{~Hz}, 6 \mathrm{H}) ;{ }^{13} \mathrm{C} \mathrm{NMR}(126 \mathrm{MHz}$, $\left.\mathrm{CDCl}_{3}\right) \delta 144.1,135.9,130.8,130.3,129.8,120.5,44.9,32.7,31.0,19.8,19.6$; FT-IR (ATR) v 3087, 3019, 2956, 2925, 1463, 1442, 880, $785 \mathrm{~cm}^{-1}$; HRMS (FI) calcd for $\mathrm{C}_{11} \mathrm{H}_{16}\left(\mathrm{M}^{+}\right)$: 148.1252, found: 148.1245 . 


\section{Cycloheptatriene 8c}

$\underbrace{11}_{P h}$

8c

According to procedure $\mathrm{D}$ (reaction time: $30 \mathrm{~min}), 7 \mathbf{c}(E / Z=2.6: 1,27.0 \mathrm{mg}, 99.5 \mu \mathrm{mol})$ and NaHMDS (1.10 mol/L in THF, $182 \mu \mathrm{L}, 200 \mu \mathrm{mol})$ gave $8 \mathrm{c}(12.0 \mathrm{mg}, 65.8 \mu \mathrm{mol}, 66 \%$ yield) as a yellow oil.

8c: ${ }^{1} \mathrm{H}$ NMR $\left(500 \mathrm{MHz}, \mathrm{CDCl}_{3}\right) \delta 7.31(\mathrm{t}, J=7.4 \mathrm{~Hz}, 2 \mathrm{H}), 7.22-7.20(\mathrm{~m}, 3 \mathrm{H}), 6.17(\mathrm{t}, J=10.9 \mathrm{~Hz}$, 2H), 5.87 (dd, $J=11.7,4.3 \mathrm{~Hz}, 1 \mathrm{H}), 5.78-5.73(\mathrm{~m}, 1 \mathrm{H}), 5.17(\mathrm{~s}, 1 \mathrm{H}), 5.15(\mathrm{~s}, 1 \mathrm{H}), 3.78$ (br 1H), 2.66-2.60 (m, 1H), 2.56-2.51 (m, 1H); $\left.{ }^{13} \mathrm{C} \mathrm{NMR} \mathrm{(126} \mathrm{MHz,} \mathrm{CDCl}_{3}\right) \delta 145.9,143.6,135.3,131.3$, 130.4, 129.7, 128.5 (2C), 127.5 (2C), 126.2, 121.9, 45.4, 37.0; FT-IR (ATR) v 3084, 3060, 3022, 2925, 2893, 1492, 1452, 884, 758, $698 \mathrm{~cm}^{-1}$; HRMS (FI) calcd for $\mathrm{C}_{14} \mathrm{H}_{14}\left(\mathrm{M}^{+}\right)$: 182.1096, found: 182.1093.

\section{Cycloheptatriene 13a}

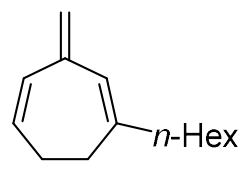

$13 a$

According to procedure D (reaction time: $1 \mathrm{~h}), \mathbf{1 2 a}(27.3 \mathrm{mg}, 97.7 \mu \mathrm{mol})$ and KHMDS $(1.0 \mathrm{~mol} / \mathrm{L}$ in THF, $200 \mu \mathrm{L}, 200 \mu \mathrm{mol})$ gave 13a $(10.3 \mathrm{mg}, 54.1 \mu \mathrm{mol}$, $55 \%$ yield) as a colorless oil.

13a: ${ }^{1} \mathrm{H}$ NMR (500 MHz, $\left.\mathrm{CDCl}_{3}\right) \delta 6.05(\mathrm{~d}, J=11.5 \mathrm{~Hz}, 1 \mathrm{H}), 5.93(\mathrm{~s}, 1 \mathrm{H}), 5.83-5.80(\mathrm{~m}, 1 \mathrm{H}), 4.96$ (d, $J=4.6 \mathrm{~Hz}, 2 \mathrm{H}), 2.30$ (d, $J=2.9 \mathrm{~Hz}, 4 \mathrm{H}), 2.07$ (t, $J=7.4 \mathrm{~Hz}, 2 \mathrm{H}), 1.44-1.40$ (m, 2H), 1.32-1.25 $(\mathrm{m}, 6 \mathrm{H}), 0.88(\mathrm{t}, J=6.6 \mathrm{~Hz}, 3 \mathrm{H}) ;{ }^{13} \mathrm{C} \mathrm{NMR}\left(126 \mathrm{MHz}, \mathrm{CDCl}_{3}\right) \delta 144.6,143.9,131.5,130.9,125.8$, 119.1, 41.5, 32.2, 31.8, 29.1, 27.9 (2C), 22.6, 14.1; FT-IR (ATR) v 3086, 3019, 2925, 2854, 1567, 1457, 1435, $876 \mathrm{~cm}^{-1}$; HRMS (FI) calcd for $\mathrm{C}_{14} \mathrm{H}_{22}\left(\mathrm{M}^{+}\right)$: 190.1722, found: 190.1723 .

\section{Cycloheptatriene 13b}

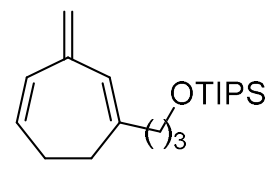

$13 b$

According to procedure D (reaction time: $1 \mathrm{~h}), \mathbf{1 2 b}(40.7 \mathrm{mg}, 99.3 \mu \mathrm{mol})$ and KHMDS $(1.0 \mathrm{~mol} / \mathrm{L}$ in THF, $200 \mu \mathrm{L}, 200 \mu \mathrm{mol})$ gave $13 \mathrm{~b}(18.8 \mathrm{mg}, 58.6 \mu \mathrm{mol}$, $59 \%$ yield) as a colorless oil.

13b: ${ }^{1} \mathrm{H}$ NMR $\left(500 \mathrm{MHz}, \mathrm{CDCl}_{3}\right) \delta 6.05(\mathrm{~d}, J=11.5 \mathrm{~Hz}, 1 \mathrm{H}), 5.95(\mathrm{~s}, 1 \mathrm{H}), 5.81(\mathrm{br}, 1 \mathrm{H}), 4.96(\mathrm{~s}$, 2H), $3.69(\mathrm{t}, J=6.3 \mathrm{~Hz}, 2 \mathrm{H}), 2.31(\mathrm{~s}, 4 \mathrm{H}), 2.16(\mathrm{t}, J=7.4 \mathrm{~Hz}, 2 \mathrm{H}), 1.71-1.65(\mathrm{~m}, 2 \mathrm{H}), 1.11-1.05$ $(\mathrm{m}, 21 \mathrm{H}) ;{ }^{13} \mathrm{C} \mathrm{NMR}\left(126 \mathrm{MHz}, \mathrm{CDCl}_{3}\right) \delta 144.0,143.8,131.5,130.9,126.0,119.3,62.9,37.7,32.3$, 31.3, 27.9, 18.0 (6C), 12.0 (3C); FT-IR (ATR) v 3019, 2941, 2864, 1463, 1103, $880 \mathrm{~cm}^{-1}$; HRMS (FD) calcd for $\mathrm{C}_{20} \mathrm{H}_{36} \mathrm{OSi}\left(\mathrm{M}^{+}\right)$: 320.2535 , found: 320.2547 . 


\section{Cycloheptatriene 13c}

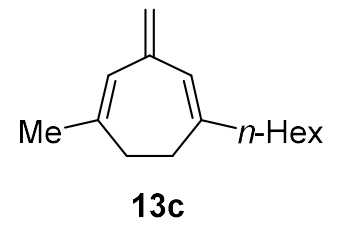

According to procedure D (reaction time: $1.5 \mathrm{~h}), \mathbf{1 2 c}(29.1 \mathrm{mg}, 99.2 \mu \mathrm{mol})$ and KHMDS (1.0 mol/L in THF, $200 \mu \mathrm{L}, 200 \mu \mathrm{mol})$ gave 13c $(11.3 \mathrm{mg}, 55.3$ $\mu \mathrm{mol}, 56 \%$ yield) as a colorless oil.

13c: ${ }^{1} \mathrm{H}$ NMR $\left(500 \mathrm{MHz}, \mathrm{CDCl}_{3}\right) \delta 5.92(\mathrm{~s}, 1 \mathrm{H}), 5.89(\mathrm{~s}, 1 \mathrm{H}), 4.85(\mathrm{~s}, 2 \mathrm{H}), 2.26(\mathrm{~s}, 4 \mathrm{H}), 2.05(\mathrm{t}, J=$ $7.4 \mathrm{~Hz}, 2 \mathrm{H}), 1.82(\mathrm{~s}, 3 \mathrm{H}), 1.43-1.39(\mathrm{~m}, 2 \mathrm{H}), 1.30-1.28(\mathrm{~m}, 6 \mathrm{H}), 0.88(\mathrm{t}, J=6.9 \mathrm{~Hz}, 3 \mathrm{H}) ;{ }^{13} \mathrm{C} \mathrm{NMR}$ $\left(126 \mathrm{MHz} \mathrm{CDCl}_{3}\right) \delta 144.2,143.5,140.3,126.1,125.7,117.2,41.3,33.1,31.8,31.3,29.1,27.9,27.4$, 22.6, 14.1; FT-IR (ATR) v 2956, 2924, 2853, 1566, 1433, $876 \mathrm{~cm}^{-1}$; HRMS (FI) calcd for $\mathrm{C}_{15} \mathrm{H}_{24}$ $\left(\mathrm{M}^{+}\right):$204.1878, found: 204.1884 .

\section{Cycloheptatriene 13d}

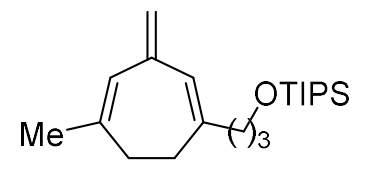

$13 d$

According to procedure D (reaction time: $2 \mathrm{~h}$ ), 12d (20.8 $\mathrm{mg}, 49.1 \mu \mathrm{mol})$ and KHMDS (1.0 mol/L in THF, $100 \mu \mathrm{L}, 100 \mu \mathrm{mol})$ gave 13d $(6.8 \mathrm{mg}, 20.3$ $\mu \mathrm{mol}, 41 \%$ yield) as a colorless oil.

13d: ${ }^{1} \mathrm{H}$ NMR $\left(500 \mathrm{MHz}, \mathrm{CDCl}_{3}\right) \delta 5.92(\mathrm{~s}, 2 \mathrm{H}), 4.85(\mathrm{~s}, 2 \mathrm{H}), 3.68(\mathrm{t}, J=6.6 \mathrm{~Hz}, 2 \mathrm{H}), 2.29-2.22$ $(\mathrm{m}, 4 \mathrm{H}), 2.14(\mathrm{t}, J=7.7 \mathrm{~Hz}, 2 \mathrm{H}), 1.82(\mathrm{~s}, 3 \mathrm{H}), 1.70-1.64(\mathrm{~m}, 2 \mathrm{H}), 1.10-1.05(\mathrm{~m}, 21 \mathrm{H}) ;{ }^{13} \mathrm{C} \mathrm{NMR}$ $\left(126 \mathrm{MHz}, \mathrm{CDCl}_{3}\right) \delta 143.6,143.4,140.3,126.1,125.9,117.4,62.9,37.5,33.1,31.4,31.3,27.4,18.0$ (6C), 12.0 (3C); FT-IR (ATR) v 2941, 2865, 1463, 1102, 880, $679 \mathrm{~cm}^{-1}$; HRMS (FD) calcd for $\mathrm{C}_{21} \mathrm{H}_{38} \mathrm{OSi}\left(\mathrm{M}^{+}\right)$: 334.2692, found: 334.2688.

\section{Cycloheptatriene 15}

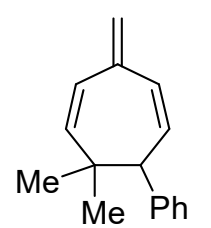

According to procedure $\mathrm{D}$ [reaction time: $15 \mathrm{~min}$, under diluted condition $(0.01$ $\mathrm{mol} / \mathrm{L})], 14(E / Z=1.2: 1,22.2 \mathrm{mg}, 74.1 \mu \mathrm{mol})$ and KHMDS $(1.0 \mathrm{~mol} / \mathrm{L}$ in THF, 77.9 $\mu \mathrm{L}, 77.9 \mu \mathrm{mol})$ gave $15(7.9 \mathrm{mg}, 37.6 \mu \mathrm{mol}, 51 \%$ yield $)$ as a colorless oil.

15

15: ${ }^{1} \mathrm{H}$ NMR (500 MHz, $\left.\mathrm{CDCl}_{3}\right) \delta 7.24-7.18(\mathrm{~m}, 5 \mathrm{H}), 6.19(\mathrm{~d}, J=12.0 \mathrm{~Hz}, 1 \mathrm{H}), 6.07(\mathrm{dd}, J=12.0$, $1.1 \mathrm{~Hz}, 1 \mathrm{H}), 5.83(\mathrm{dd}, J=12.0,7.0 \mathrm{~Hz}, 1 \mathrm{H}), 5.44(\mathrm{~d}, J=12.0 \mathrm{~Hz}, 1 \mathrm{H}), 5.19(\mathrm{~d}, J=3.4 \mathrm{~Hz}, 2 \mathrm{H})$, $3.44(\mathrm{~d}, J=7.0 \mathrm{~Hz}, 1 \mathrm{H}), 1.16(\mathrm{~s}, 3 \mathrm{H}), 0.96(\mathrm{~s}, 3 \mathrm{H}) ;{ }^{13} \mathrm{C} \mathrm{NMR}\left(126 \mathrm{MHz}, \mathrm{CDCl}_{3}\right) \delta 143.3,142.5$, 140.2, 132.7, 130.0, 129.6, 128.3, 127.7 (2C), 126.4 (2C), 122.0, 57.2, 40.1, 31.5, 29.0; FT-IR (ATR) $v$ 3084, 3060, 3019, 2956, 2868, 1452, 885, $699 \mathrm{~cm}^{-1}$; HRMS (FI) calcd for $\mathrm{C}_{16} \mathrm{H}_{18}\left(\mathrm{M}^{+}\right): 210.1409$, found: 210.1402 . 


\section{Cycloheptatriene 17}

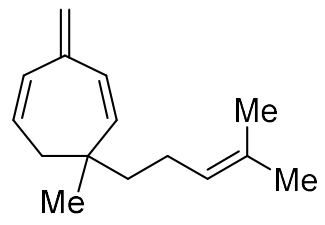

17

According to procedure $\mathrm{D}$ (reaction time: $1.5 \mathrm{~h}), \mathbf{1 6}[(2 E, 4 E+2 E, 4 Z):(2 Z, 4 E$ $+2 Z, 4 Z)=10: 1,58.9 \mathrm{mg}, 202 \mu \mathrm{mol}]$ and NaHMDS $(1.10 \mathrm{~mol} / \mathrm{L}$ in THF, 364 $\mu \mathrm{L}, 400 \mu \mathrm{mol})$ gave 17 (23.0 mg, $114 \mu \mathrm{mol}, 56 \%$ yield) as a colorless oil.

17: ${ }^{1} \mathrm{H}$ NMR $\left(500 \mathrm{MHz}, \mathrm{CDCl}_{3}\right) \delta 6.12(\mathrm{~d}, J=11.5 \mathrm{~Hz}, 1 \mathrm{H}), 5.95(\mathrm{~d}, J=12.0 \mathrm{~Hz}, 1 \mathrm{H}), 5.71-5.67$ (m, 1H), $5.58(\mathrm{~d}, J=12.0 \mathrm{~Hz}, 1 \mathrm{H}), 5.10-5.03(\mathrm{~m}, 3 \mathrm{H}), 2.30$ (dd, $J=15.9,6.6 \mathrm{~Hz}, 1 \mathrm{H}), 2.19$ (dd, $J$ $=15.9,5.7 \mathrm{~Hz}, 1 \mathrm{H}), 1.98-1.93(\mathrm{~m}, 2 \mathrm{H}), 1.67(\mathrm{~s}, 3 \mathrm{H}), 1.59(\mathrm{~s}, 3 \mathrm{H}), 1.42-1.39(\mathrm{~m}, 2 \mathrm{H}), 1.05(\mathrm{~s}, 3 \mathrm{H})$; ${ }^{13} \mathrm{C}$ NMR $\left(126 \mathrm{MHz}, \mathrm{CDCl}_{3}\right) \delta 143.6,141.0,131.2,131.0,128.6,127.9,124.8,120.9,42.4,39.4$, 39.3, 27.8, 25.7, 23.0, 17.6; FT-IR (ATR) v 3087, 3017, 2966, 2913, 1566, 1454, 1442, 1375, 882 $\mathrm{cm}^{-1}$; HRMS (FI) calcd for $\mathrm{C}_{15} \mathrm{H}_{22}\left(\mathrm{M}^{+}\right)$: 202.1722, found: 202.1718 .

\section{Cycloheptatriene 19a}

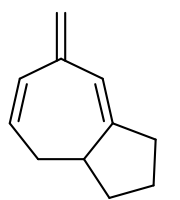

$19 a$

According to procedure D (reaction time: $2 \mathrm{~h}), \mathbf{1 8 a}(46.7 \mathrm{mg}, 198 \mu \mathrm{mol})$ and NaHMDS $(1.10 \mathrm{~mol} / \mathrm{L}$ in THF, $364 \mu \mathrm{L}, 200 \mu \mathrm{mol})$ gave, after purification by flash column chromatography [ $\mathrm{SiO}_{2}$ (neutralized by elution of $N, N$-dimethylaniline), pentane only], 19a (19.9 mg, $136 \mu \mathrm{mol}, 69 \%$ yield) as a colorless oil.

19a: ${ }^{1} \mathrm{H}$ NMR $\left(500 \mathrm{MHz}, \mathrm{CDCl}_{3}\right) \delta$ 6.06-6.04 (m, 2H), 5.79-5.74 (m, 1H), $4.94(\mathrm{~s}, 1 \mathrm{H}), 4.93(\mathrm{~s}, 1 \mathrm{H})$, 2.73-2.67 (m, 1H), 2.46-2.43 (m, 2H), $2.35(\mathrm{qd}, J=8.2,2.9 \mathrm{~Hz}, 1 \mathrm{H}), 2.23-2.17(\mathrm{~m}, 1 \mathrm{H}), 1.99-1.94$ $(\mathrm{m}, 1 \mathrm{H}), 1.76-1.70(\mathrm{~m}, 1 \mathrm{H}), 1.58-1.49(\mathrm{~m}, 1 \mathrm{H}), 1.35-1.25(\mathrm{~m}, 1 \mathrm{H}) ;{ }^{13} \mathrm{C} \mathrm{NMR}\left(\mathrm{CDCl}_{3}, 126 \mathrm{MHz}\right) \delta$ 150.9, 143.4, 131.0, 130.0, 121.4, 118.6, 43.4, 35.8, 35.4, 33.9, 25.1; FT-IR (ATR) v 3085, 3017, 2950, 2928, 2866, 1566, 1448, 1431, $876 \mathrm{~cm}^{-1}$; HRMS (FI) calcd for $\mathrm{C}_{11} \mathrm{H}_{14}\left(\mathrm{M}^{+}\right)$: 146.1096, found: 146.1089 .

\section{Cycloheptatriene 19b}

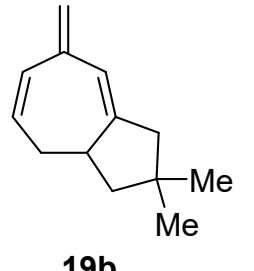

According to procedure D (reaction time: $2 \mathrm{~h}), \mathbf{1 8 b}(28.3 \mathrm{mg}, 107 \mu \mathrm{mol})$ and NaHMDS (1.10 mol/L in THF, $195 \mu \mathrm{L}, 215 \mu \mathrm{mol})$ gave 19b $(11.8 \mathrm{mg}, 67.7 \mu \mathrm{mol}$, $63 \%$ yield) as a colorless oil.

19b: ${ }^{1} \mathrm{H}$ NMR $\left(500 \mathrm{MHz}, \mathrm{CDCl}_{3}\right) \delta 6.04(\mathrm{~d}, J=11.5 \mathrm{~Hz}, 1 \mathrm{H}), 6.01(\mathrm{~s}, 1 \mathrm{H}), 5.78-5.74(\mathrm{~m}, 1 \mathrm{H}), 4.94$ $(\mathrm{s}, 2 \mathrm{H}), 2.94-2.88(\mathrm{~m}, 1 \mathrm{H}), 2.34-2.27(\mathrm{~m}, 2 \mathrm{H}), 2.23-2.18(\mathrm{~m}, 2 \mathrm{H}), 1.72-1.67(\mathrm{~m}, 1 \mathrm{H}), 1.24(\mathrm{t}, J=$ $11.2 \mathrm{~Hz}, 1 \mathrm{H}), 1.06$ (s, 3H), 0.96 (s, 3H); ${ }^{13} \mathrm{C} \mathrm{NMR}\left(126 \mathrm{MHz}, \mathrm{CDCl}_{3}\right) \delta 151.0,143.4,131.0,130.1$, 
122.1, 118.7, 50.0, 48.8, 42.3, 37.6, 34.2, 28.8, 27.2; FT-IR (ATR) v 3085, 3017, 2951, 2925, 2866, 1566, 1460, 1428, 1366, $874 \mathrm{~cm}^{-1}$; HRMS (FI) calcd for $\mathrm{C}_{13} \mathrm{H}_{18}\left(\mathrm{M}^{+}\right)$: 174.1409, found: 174.1402 .

\section{Cycloheptatriene 21a}

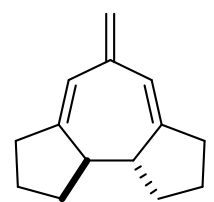

$21 \mathrm{a}$

According to procedure D (reaction time: $1.5 \mathrm{~h}), \mathbf{2 0 a}(216 \mathrm{mg}, 785 \mu \mathrm{mol})$ and KHMDS (1.0 mol/L in THF, $1.57 \mathrm{~mL}, 1.57 \mathrm{mmol})$ gave $21 \mathrm{a}(70.1 \mathrm{mg}, 376 \mu \mathrm{mol}$, $48 \%$ yield) as a yellow oil.

21a: ${ }^{1} \mathrm{H}$ NMR $\left(500 \mathrm{MHz}, \mathrm{CDCl}_{3}\right) \delta 6.05(\mathrm{~s}, 2 \mathrm{H}), 4.81(\mathrm{~s}, 2 \mathrm{H}), 2.45-2.42(\mathrm{~m}, 6 \mathrm{H}), 2.02-1.98(\mathrm{~m}$, $2 \mathrm{H}), 1.77-1.71(\mathrm{~m}, 2 \mathrm{H}), 1.54-1.43(\mathrm{~m}, 2 \mathrm{H}), 1.37-1.29(\mathrm{~m}, 2 \mathrm{H}) ;{ }^{13} \mathrm{C} \mathrm{NMR}\left(126 \mathrm{MHz}, \mathrm{CDCl}_{3}\right) \delta$ 149.1, 142.3 (2C), 121.9 (2C), 116.2, 48.2 (2C), 35.6 (2C), 34.1 (2C), 24.9 (2C); FT-IR (ATR) v $3083,2951,2863,1565,1446,1429,874 \mathrm{~cm}^{-1}$; HRMS (FI) calcd for $\mathrm{C}_{14} \mathrm{H}_{18}\left(\mathrm{M}^{+}\right): 186.1409$, found: 186.1417.

\section{Cycloheptatriene 21b}

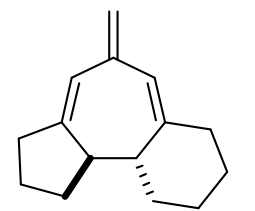

21b

According to procedure D (reaction time: $2 \mathrm{~h}), \mathbf{2 0 b}(E / Z=1.6: 1,57.8 \mathrm{mg}, 200$ $\mu \mathrm{mol})$ and KHMDS (1.0 mol/L in THF, $400 \mu \mathrm{L}, 400 \mu \mathrm{mol})$ gave $\mathbf{2 1 b}(15.8 \mathrm{mg}, 78.9$ $\mu \mathrm{mol}, 39 \%$ yield) as a colorless oil.

21b: ${ }^{1} \mathrm{H}$ NMR $\left(500 \mathrm{MHz}, \mathrm{CDCl}_{3}\right) \delta 6.10(\mathrm{~s}, 1 \mathrm{H}), 5.94(\mathrm{~s}, 1 \mathrm{H}), 4.90(\mathrm{~s}, 1 \mathrm{H}), 4.87(\mathrm{~s}, 1 \mathrm{H}), 2.55-2.50$ (m, 1H), 2.39-2.36 (m, 2H), $2.29(\mathrm{~d}, J=14.3 \mathrm{~Hz}, 1 \mathrm{H}), 2.14-2.05(\mathrm{~m}, 3 \mathrm{H}), 1.94-1.91(\mathrm{~m}, 1 \mathrm{H}), 1.76-$ $1.69(\mathrm{~m}, 3 \mathrm{H}), 1.47-1.25(\mathrm{~m}, 4 \mathrm{H}), 1.12-1.03(\mathrm{~m}, 1 \mathrm{H}) ;{ }^{13} \mathrm{C} \mathrm{NMR}\left(126 \mathrm{MHz}, \mathrm{CDCl}_{3}\right) \delta 149.3,144.0$, 143.2, 125.2, 122.7, 117.2, 47.4, 44.9, 38.3, 36.0, 34.3, 32.3, 26.1, 25.2, 24.9; FT-IR (ATR) v 3082, 2927, 2853, 1566, 1445, $876 \mathrm{~cm}^{-1}$; HRMS (FD) calcd for $\mathrm{C}_{15} \mathrm{H}_{20}\left(\mathrm{M}^{+}\right): 200.1565$, found: 200.1561 . 


\section{Structural assignment of 21 a by cyclopropanation}

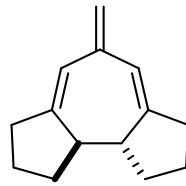

$21 \mathrm{a}$

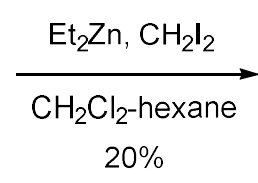

$20 \%$

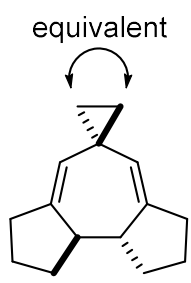

trans-S32

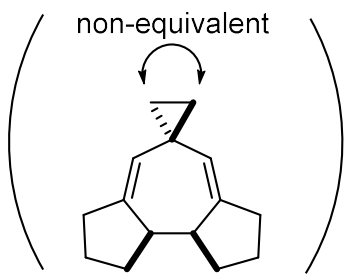

cis-S32

To a solution of $21 \mathrm{a}(18.2 \mathrm{mg}, 97.7 \mu \mathrm{mol})$ in $\mathrm{CH}_{2} \mathrm{Cl}_{2}(500 \mu \mathrm{L})$ was added $\mathrm{Et}_{2} \mathrm{Zn}(1.0 \mathrm{~mol} / \mathrm{L}$ in hexane, $150 \mu \mathrm{L}, 150 \mu \mathrm{mol})$ at $0{ }^{\circ} \mathrm{C}$. To this mixture was added $\mathrm{CH}_{2} \mathrm{I}_{2}(24.1 \mu \mathrm{L}, 300 \mu \mathrm{mol})$ dropwise, and the reaction mixture was stirred for $1 \mathrm{~h}$ at the same temperature. The reaction was quenched with saturated aqueous $\mathrm{NH}_{4} \mathrm{Cl}$ solution $(500 \mu \mathrm{L})$. Two layers were separated, and the aqueous layer was extracted with hexane $(500 \mu \mathrm{L} \times 3)$. The combined organic layer was dried over $\mathrm{MgSO}_{4}$, filtered, and concentrated under reduced pressure. The resulting residue was purified by PTLC $\left(\mathrm{R}_{\mathrm{f}}=0.5\right.$, hexane only) to afford $\mathbf{S 3 2}$ (along with several impurities, $4.0 \mathrm{mg}, 20 \mu \mathrm{mol}$, ca. 20\% yield) as a colorless oil.

S32: ${ }^{1} \mathrm{H}$ NMR $\left(500 \mathrm{MHz}, \mathrm{CDCl}_{3}\right) \delta 4.67$ (s, 2H), 2.44 (t, $\left.J=5.7 \mathrm{~Hz}, 2 \mathrm{H}\right), 2.33$ (q, $\left.J=4.8 \mathrm{~Hz}, 4 \mathrm{H}\right)$, 2.01-1.95 (m, 2H), 1.70-1.64 (m, 2H), 1.51-1.42 (m, 2H), 1.37-1.28 (m, 2H), 0.78-0.72 (m, 4H); ${ }^{13} \mathrm{C}$ NMR (126 MHz, $\left.\mathrm{CDCl}_{3}\right) \delta 143.8$ (2C), 125.7 (2C), 47.8 (2C), 35.4 (2C), 34.2 (2C), 25.0 (2C), 21.2, 18.1 (2C); FT-IR (ATR) v 3075, 2997, 2947, 2861, 1446, 1432, $870 \mathrm{~cm}^{-1}$; HRMS (FD) calcd for $\mathrm{C}_{15} \mathrm{H}_{20}\left(\mathrm{M}^{+}\right)$: 200.1565, found: 200.1566 .

\section{Transformation of cross-conjugated triene into cycloheptatrienyl cation}

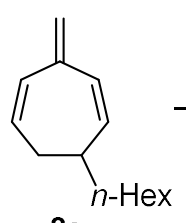

$8 \mathrm{a}$

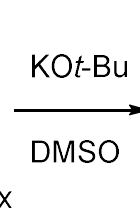

MSO

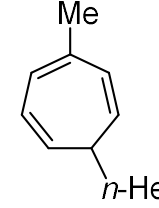

22

+ isomers

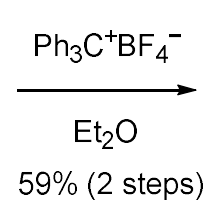$$
\text { rs }
$$

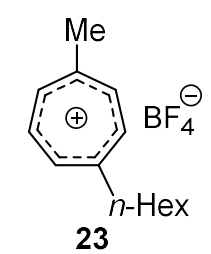

To a solution of cycloheptatriene $\mathbf{8 a}(24.1 \mathrm{mg}, 127 \mu \mathrm{mol})$ in DMSO $(1.27 \mathrm{~mL})$ was added $\mathrm{KO} t$ - $\mathrm{Bu}$ $(28.1 \mathrm{mg}, 250 \mu \mathrm{mol})$ at room temperature. After stirring for $15 \mathrm{~min}$, the reaction was quenched with aqueous $\mathrm{NH}_{4} \mathrm{Cl}$ solution $(1.0 \mathrm{~mL})$, and the mixture was extracted with $\mathrm{Et}_{2} \mathrm{O}(1.0 \mathrm{~mL} \times 5)$. The combined organic layer was washed with brine $(1.0 \mathrm{~mL})$, dried over $\mathrm{MgSO}_{4}$, filtered, and concentrated under reduced pressure. The resulting residue was filtered through a short pad of $\mathrm{SiO}_{2}$ (hexane only) and concentrated under reduced pressure to afford 22 (mixture with isomers, $18.7 \mathrm{mg}$, ca. $98.3 \mu \mathrm{mol}$, ca. $78 \%$ yield). 
To a solution of 22 and isomers $(13.5 \mathrm{mg}$, ca. $70.9 \mu \mathrm{mol})$ in dry $\mathrm{Et}_{2} \mathrm{O}(142 \mu \mathrm{L})$ was added $\mathrm{Ph}_{3} \mathrm{CBF}_{4}$ $(35.1 \mathrm{mg}, 106 \mu \mathrm{mol})$ and the reaction mixture was stirred for $17 \mathrm{~h}$ at room temperature. The solvent was then removed under reduced pressure. The resulting residue was washed with dry $\mathrm{Et}_{2} \mathrm{O}(2.0 \mathrm{~mL}$ $\times 3$ ) and concentrated under reduced pressure to afford 23 (14.9 $\mathrm{mg}, 54.0 \mu \mathrm{mol}, 76 \%$ yield) as a dark purple oil.

23: ${ }^{1} \mathrm{H}$ NMR (500 MHz, $\left.\mathrm{CDCl}_{3}\right) \delta 8.94-8.87(\mathrm{~m}, 3 \mathrm{H}), 8.84-8.82(\mathrm{~m}, 2 \mathrm{H}), 3.22(\mathrm{t}, J=8.0 \mathrm{~Hz}, 2 \mathrm{H})$, $3.10(\mathrm{~s}, 3 \mathrm{H}), 1.85-1.79(\mathrm{~m}, 2 \mathrm{H}), 1.46-1.40(\mathrm{~m}, 2 \mathrm{H}), 1.35-1.27(\mathrm{~m}, 4 \mathrm{H}), 0.89(\mathrm{t}, J=7.2 \mathrm{~Hz}, 3 \mathrm{H}) ;{ }^{13} \mathrm{C}$ NMR (126 MHz, $\left.\mathrm{CDCl}_{3}\right) \delta 174.2,170.3,154.1,153.4,153.0,152.5,151.4,42.3,32.4,31.4,29.0$, 28.6, 22.4, 14.0; FT-IR (ATR) v 3566 (br), 3056, 3013, 2955, 2928, 2858, 1494, 1052, $1032 \mathrm{~cm}^{-1}$; HRMS (FD) calcd for $\mathrm{C}_{14} \mathrm{H}_{21}\left(\mathrm{M}^{+}\right)$: 189.1643, found: 189.1639 . 
References

[1] Yoshimura, F.; Saito, H.; Abe, T.; Tanino, K. Synlett 2017, 28, 1816-1820.

[2] McCourt, R. O.; Dénès, F.; Sanchez-Sanz, G.; Scanlan, E. M. Org. Lett. 2018, 20, 2948-2951.

[3] (a) Liu, Q.; Ferreira, E. M.; Stoltz, B. M. J. Org. Chem. 2007, 72, 7352-7358. (b) Huang, S.-H.;

Tian, X.; Mi, X.; Wang, Y.; Hong, R. Tetrahedron Lett. 2015, 56, 6656-6658.

[4] Lo, J. C.; Yabe, Y; Baran, P. S. J. Am. Chem. Soc. 2014, 136, 1304-1307.

[5] Erkkilä, A.; Pihko, P. M. J. Org. Chem. 2006, 71, 2538-2541.

[6] Crimmins, M. T.; Vanier, G. S. Org. Lett. 2006, 8, 2887-2890.

[7] Kim, D. D.; Lee, S. J.; Beak, P. J. Org. Chem. 2005, 70, 5376-5386.

[8] Ozeki, M.; Egawa, H.; Kuse, A.; Takano, T.; Yasuda, N.; Mizutani, H.; Izumiya, S.; Nakashima, D.; Arimitsu, K.; Miura, T.; Kajimoto, T.; Hosoi, S.; Iwasaki, H.; Kojima, N.; Node, M.; Yamashita, M. Synthesis 2015, 47, 3392-3402. 


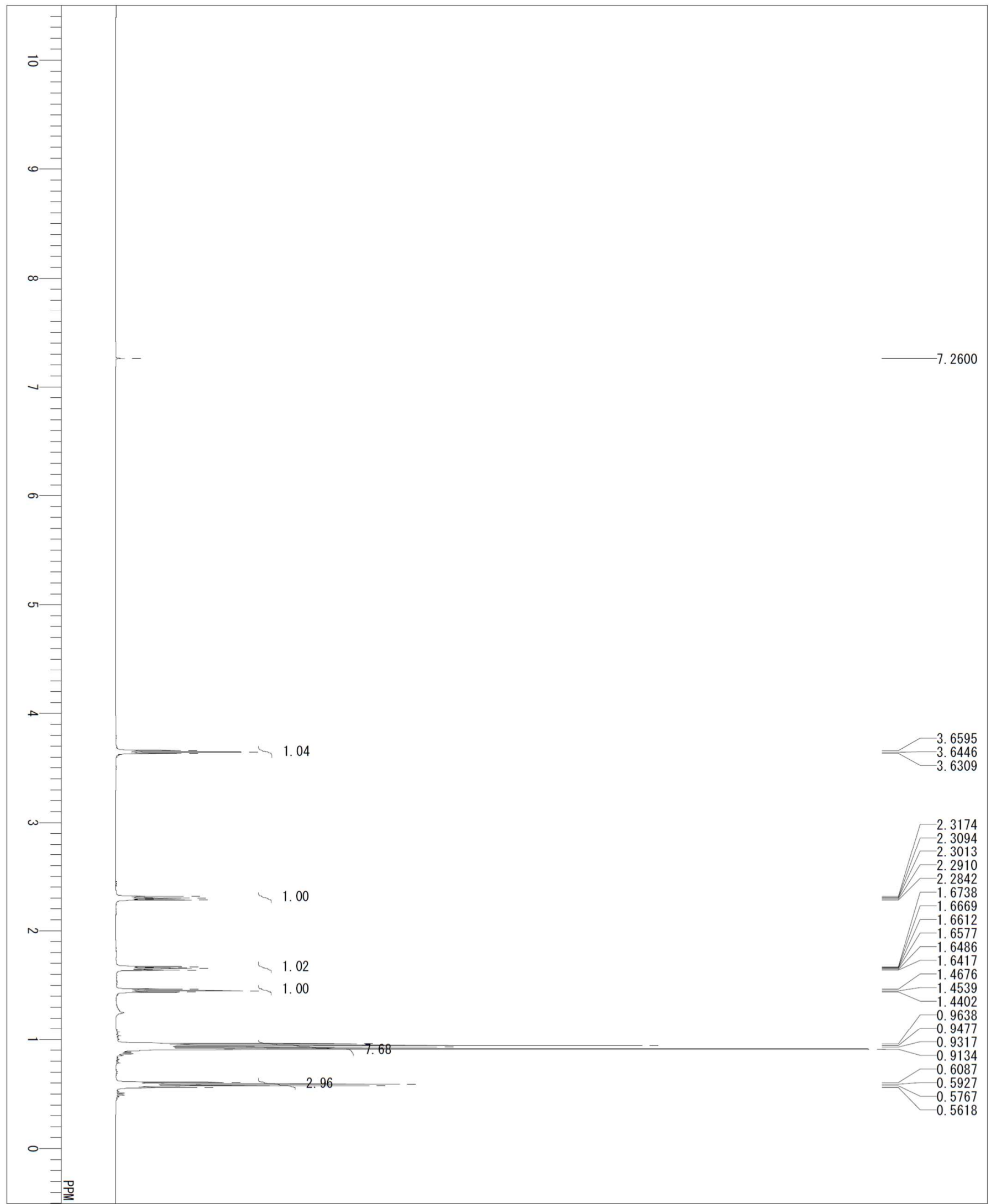

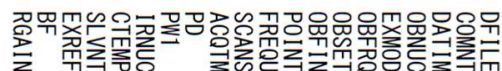

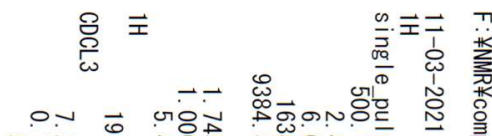

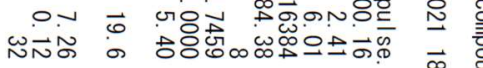

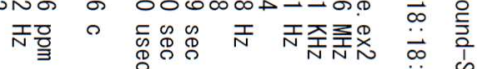




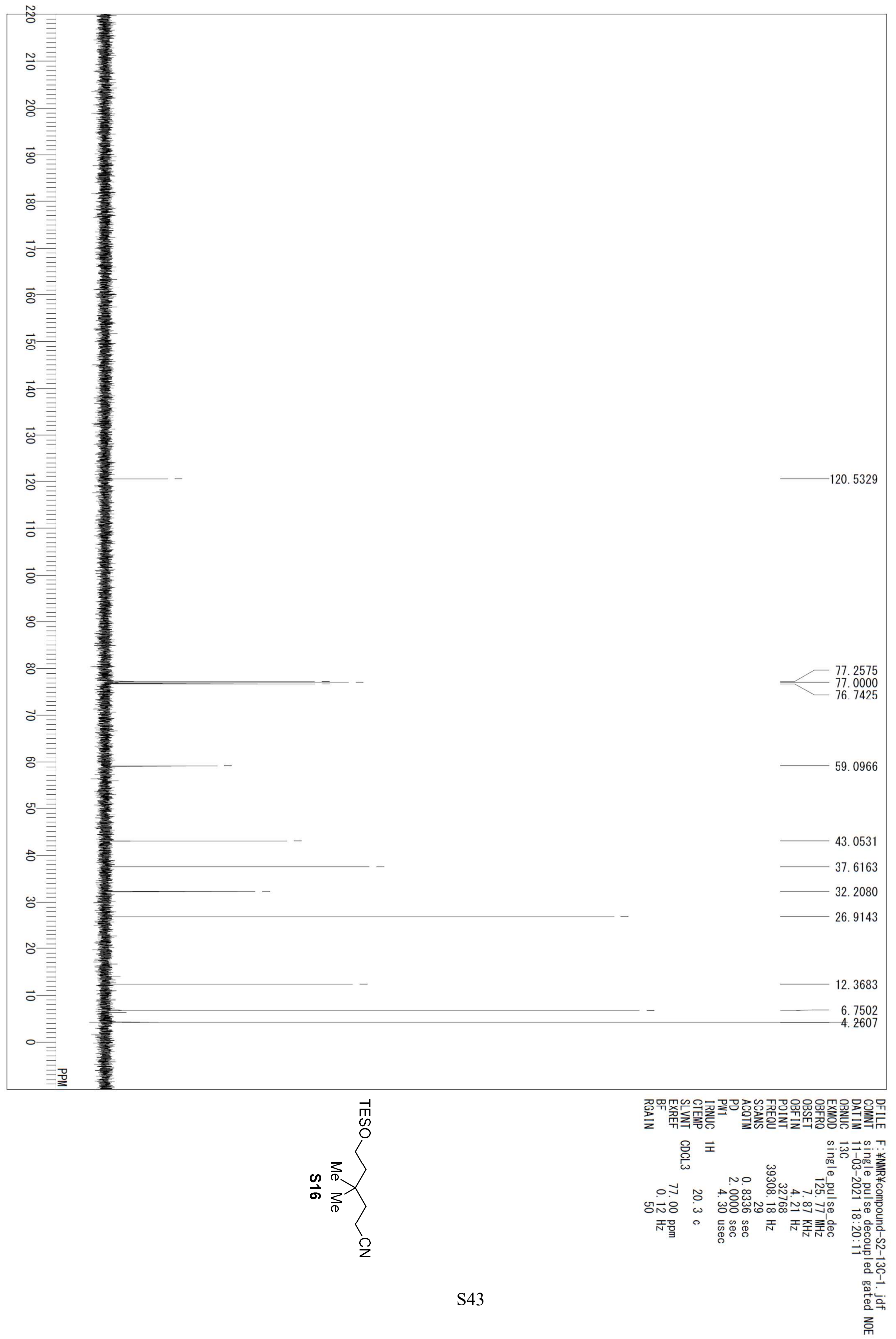




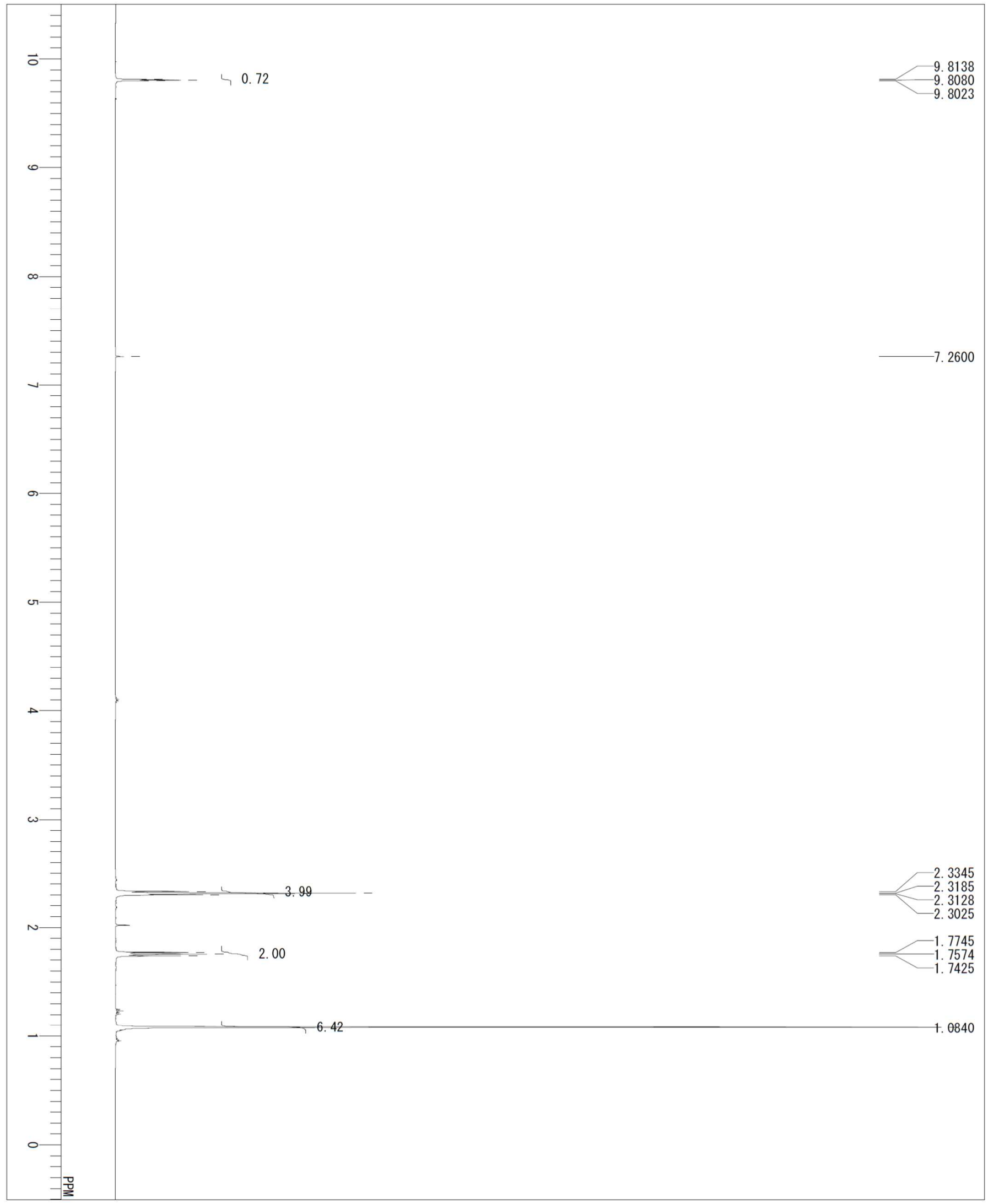

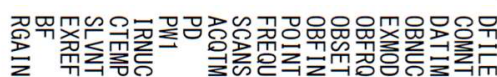

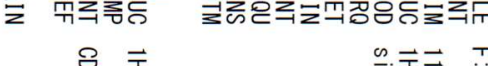

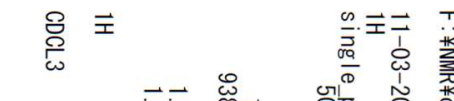

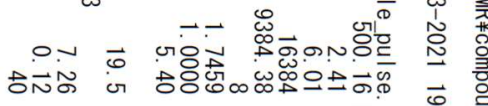

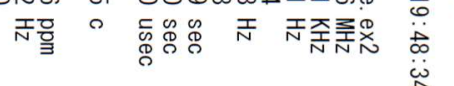




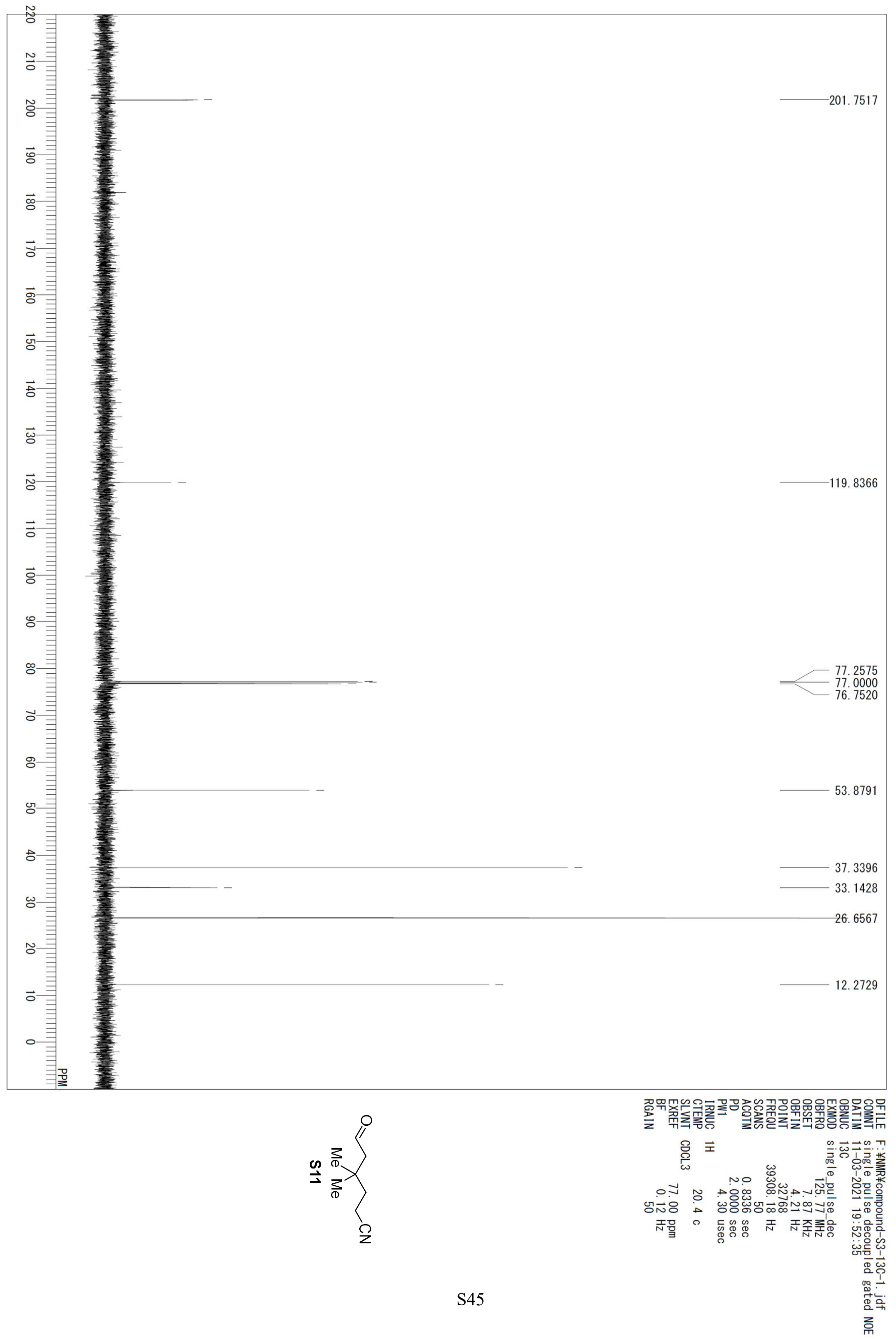




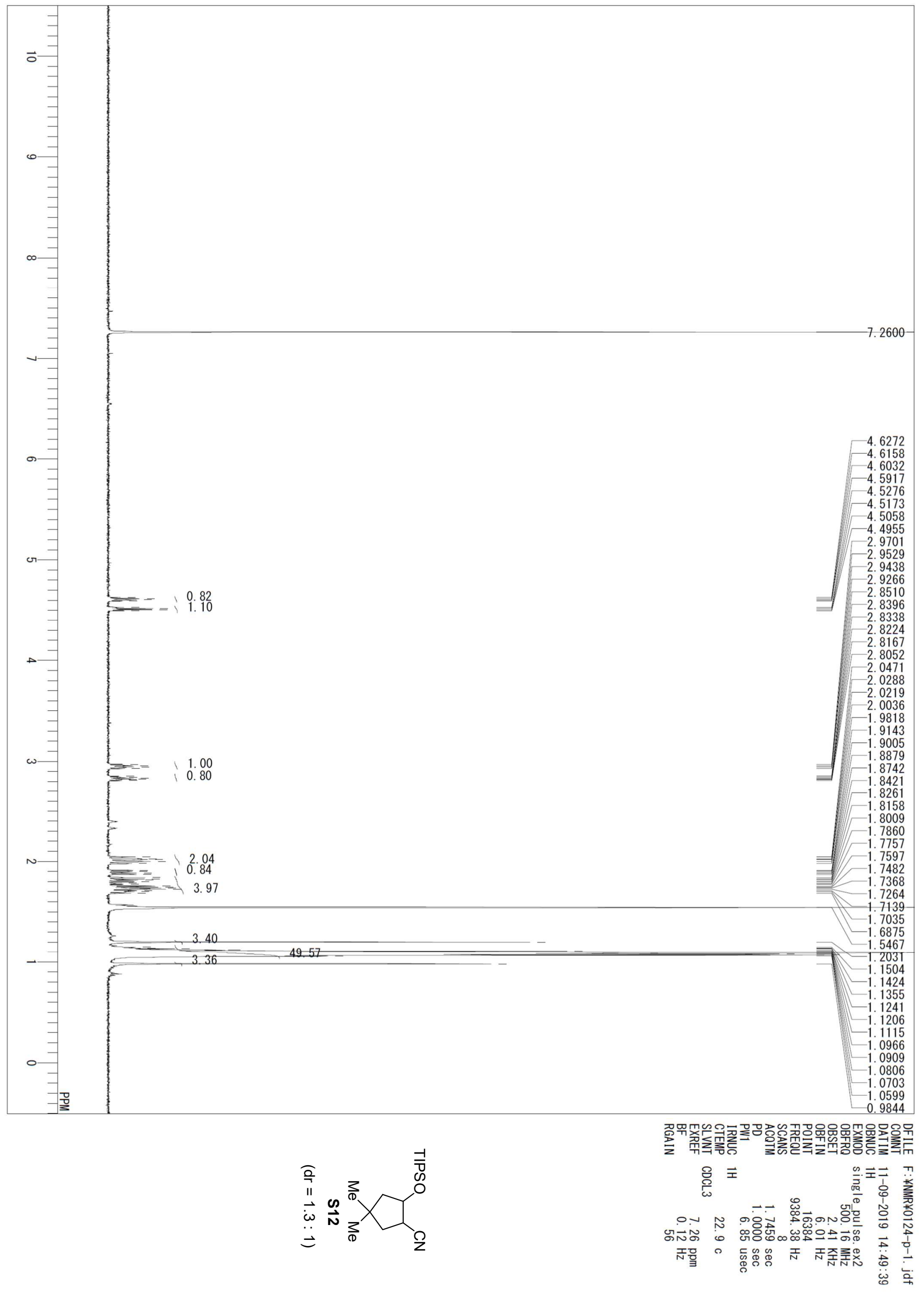




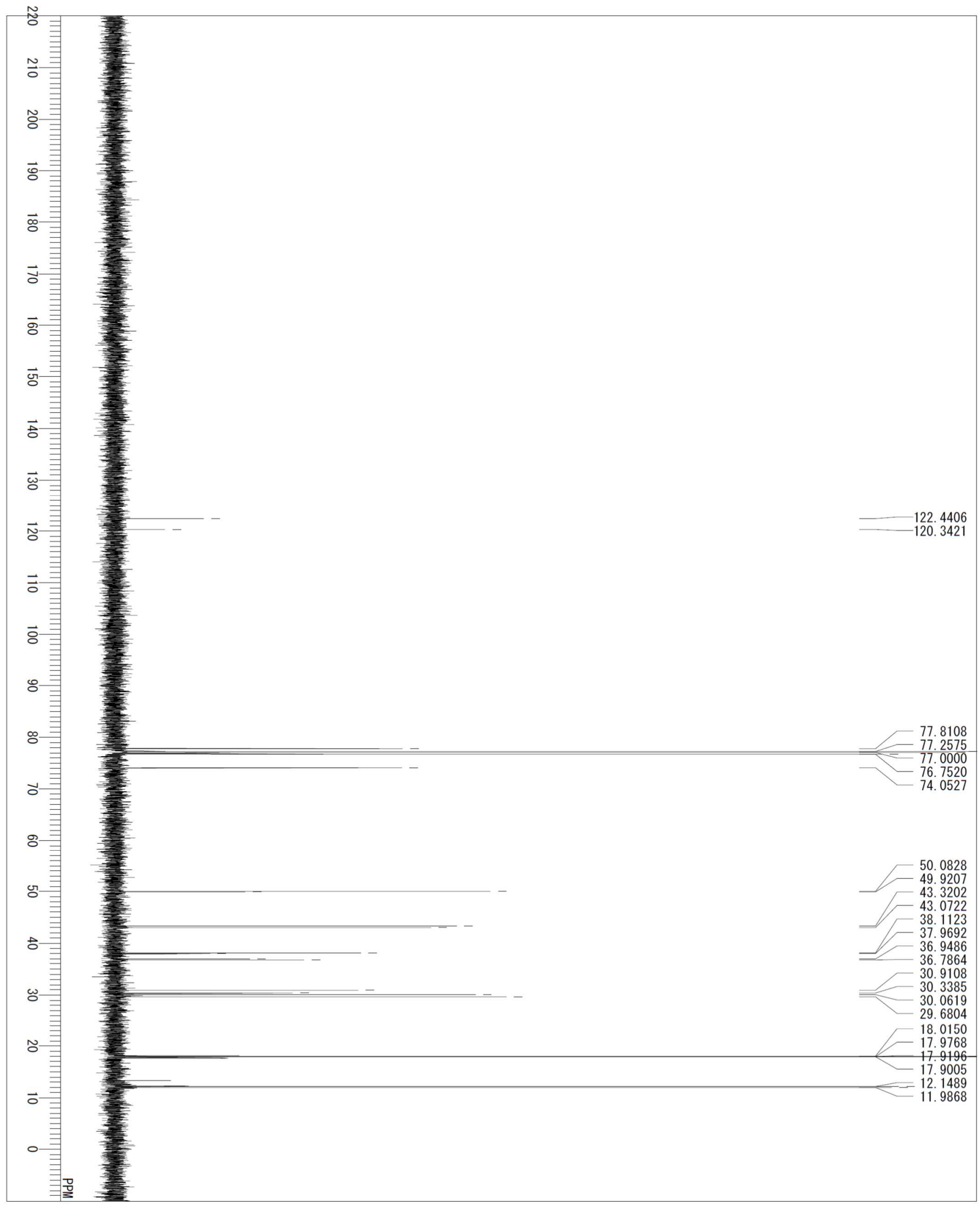

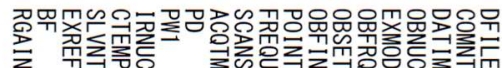

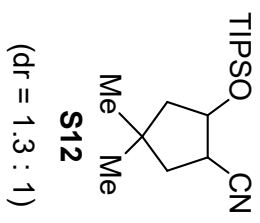

욜 표

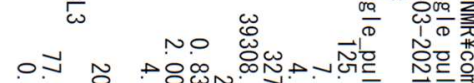

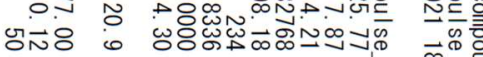

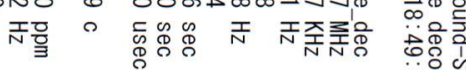



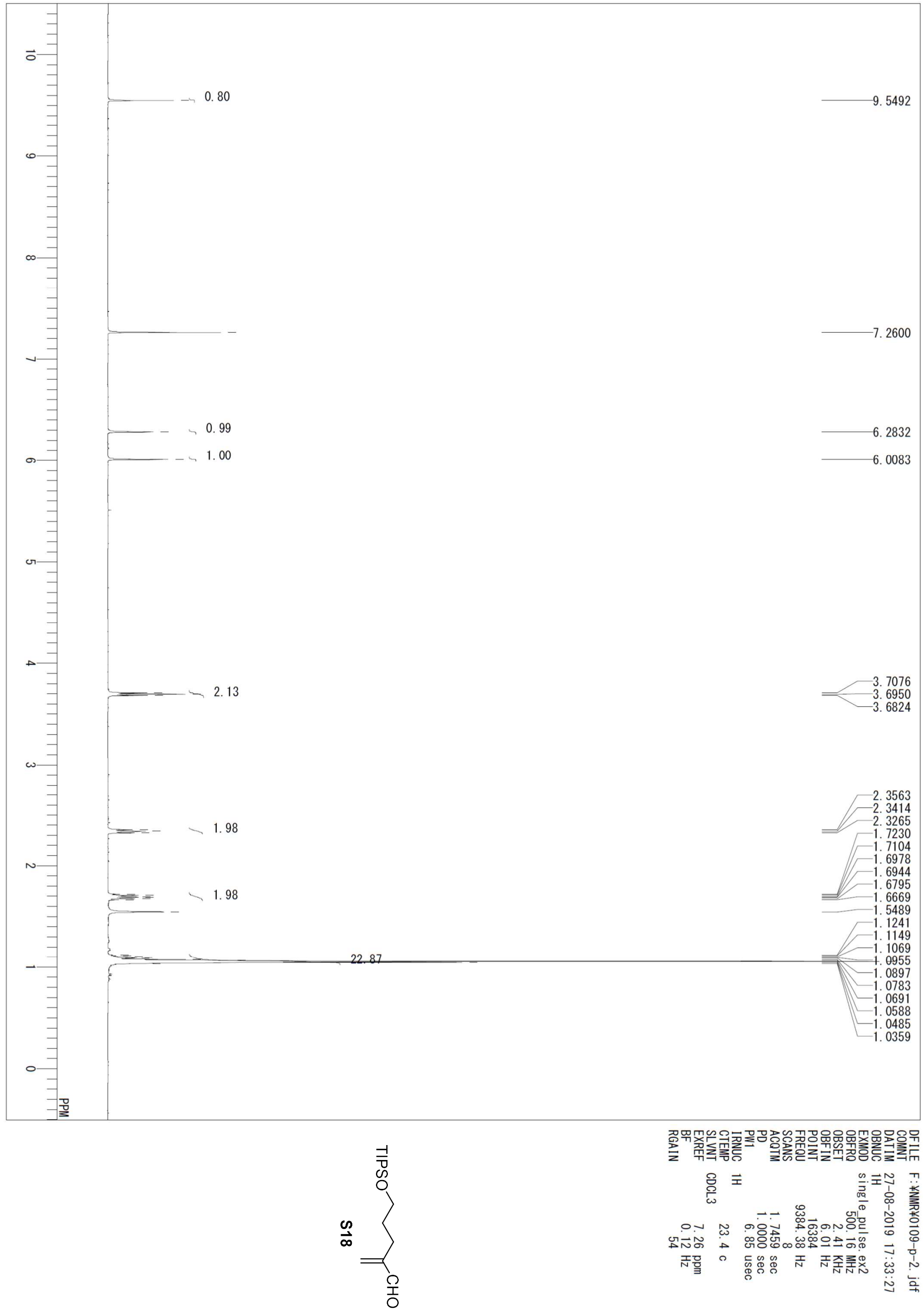

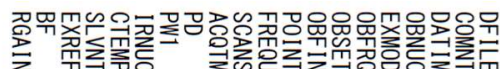

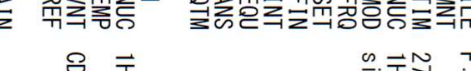
总 O. 페

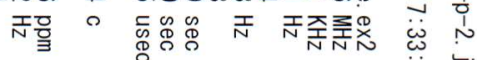

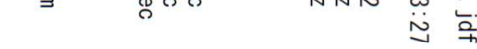




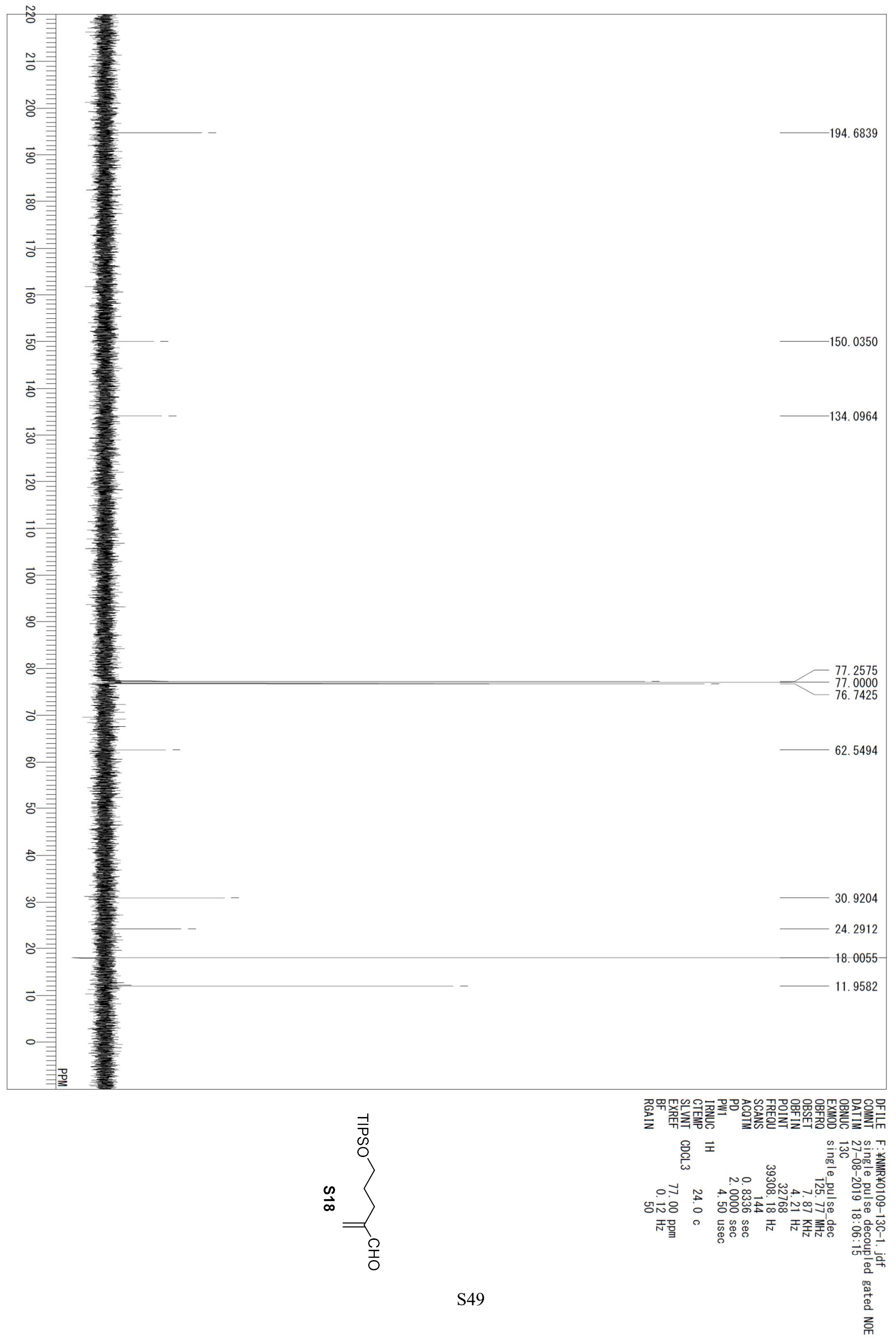



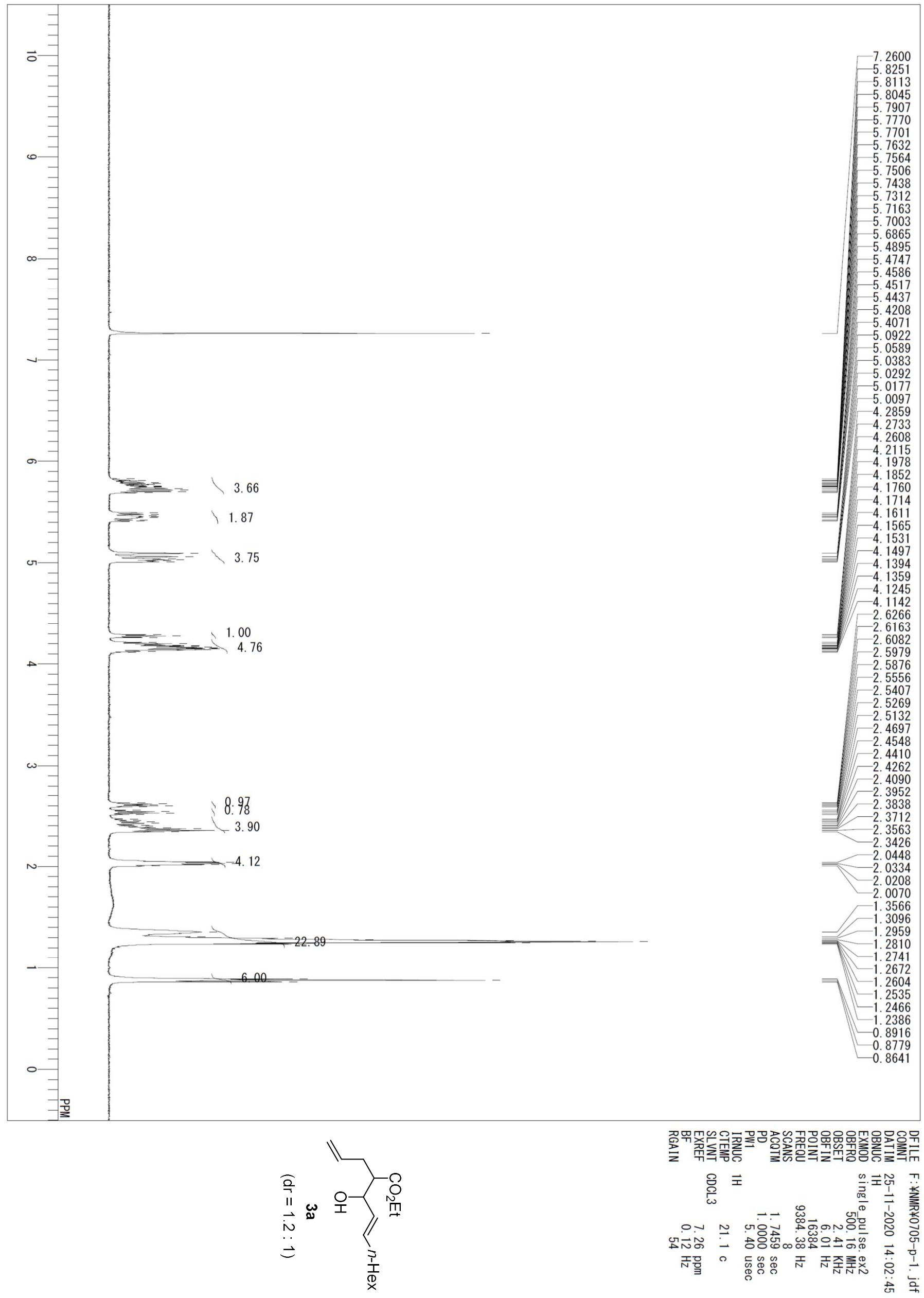

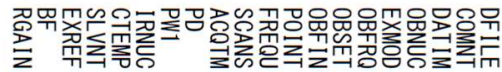

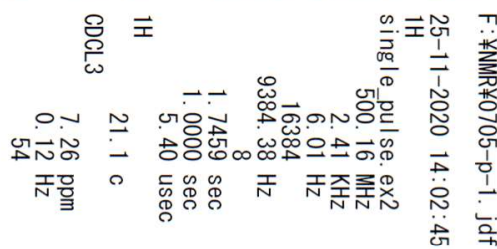



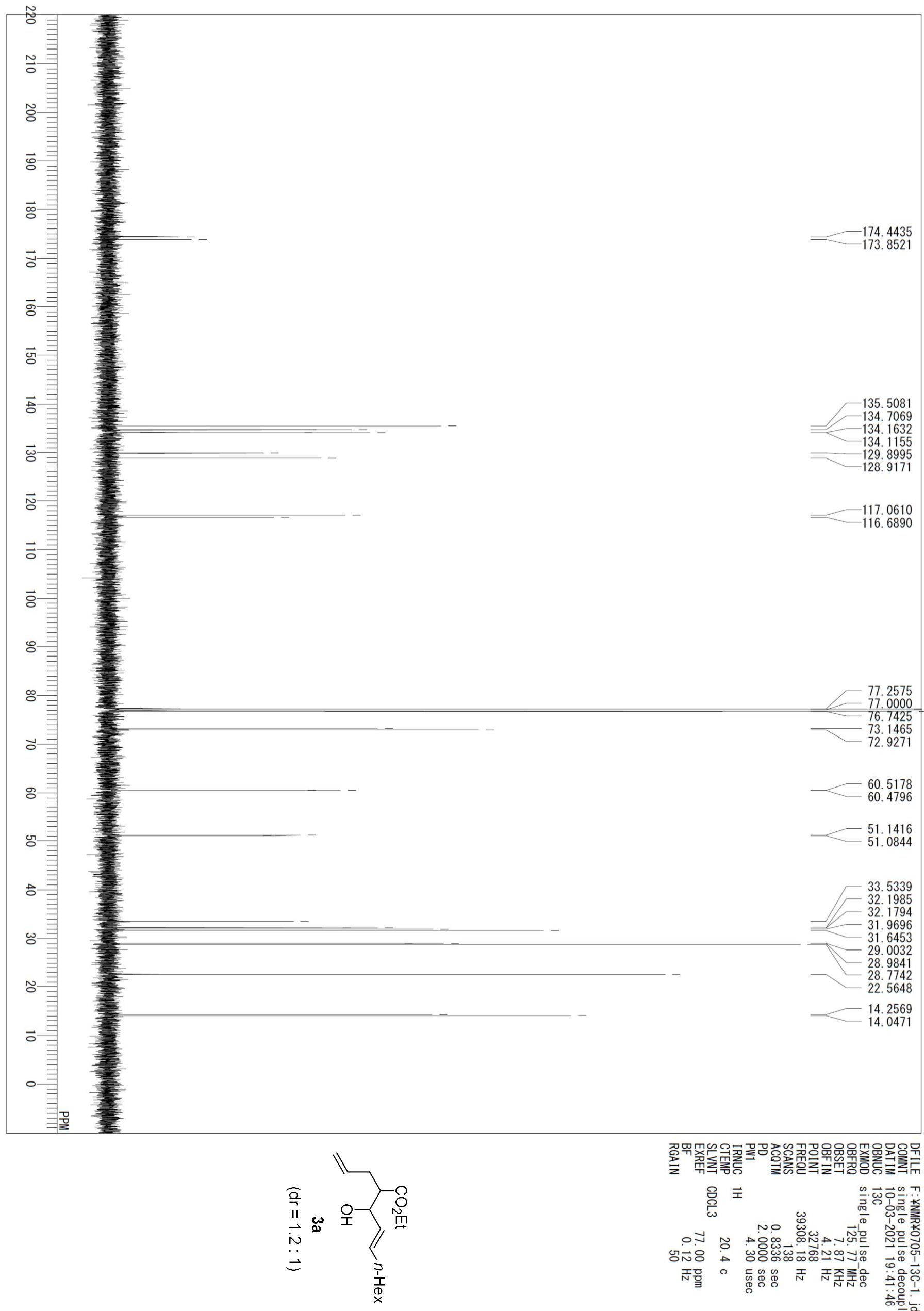

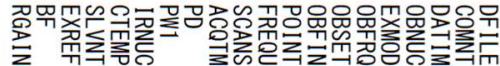

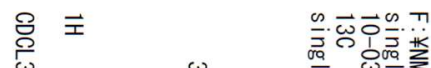

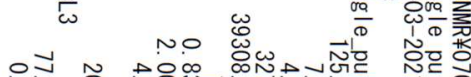
जN8

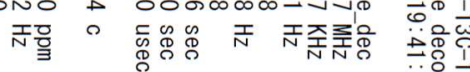
흐응 


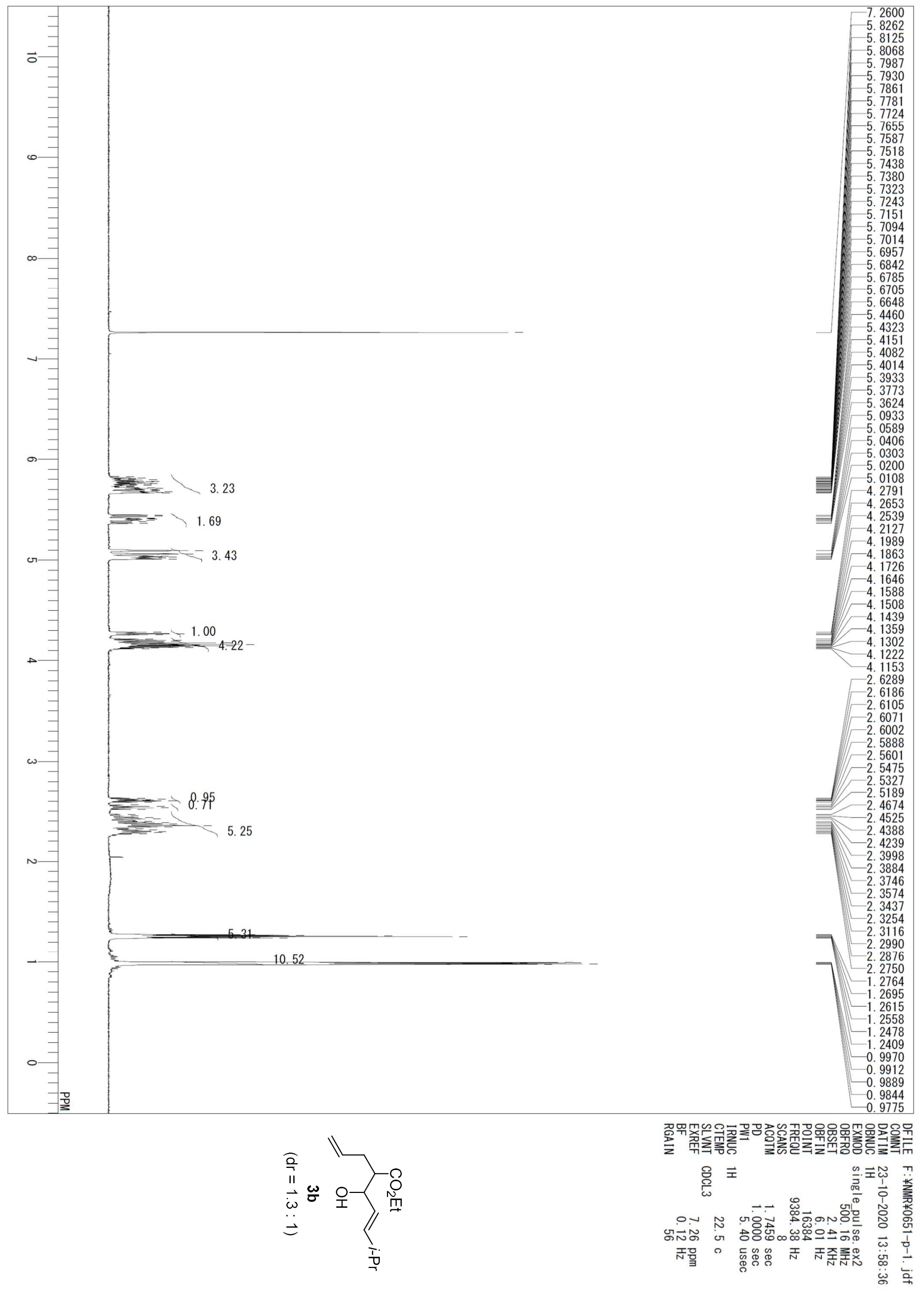



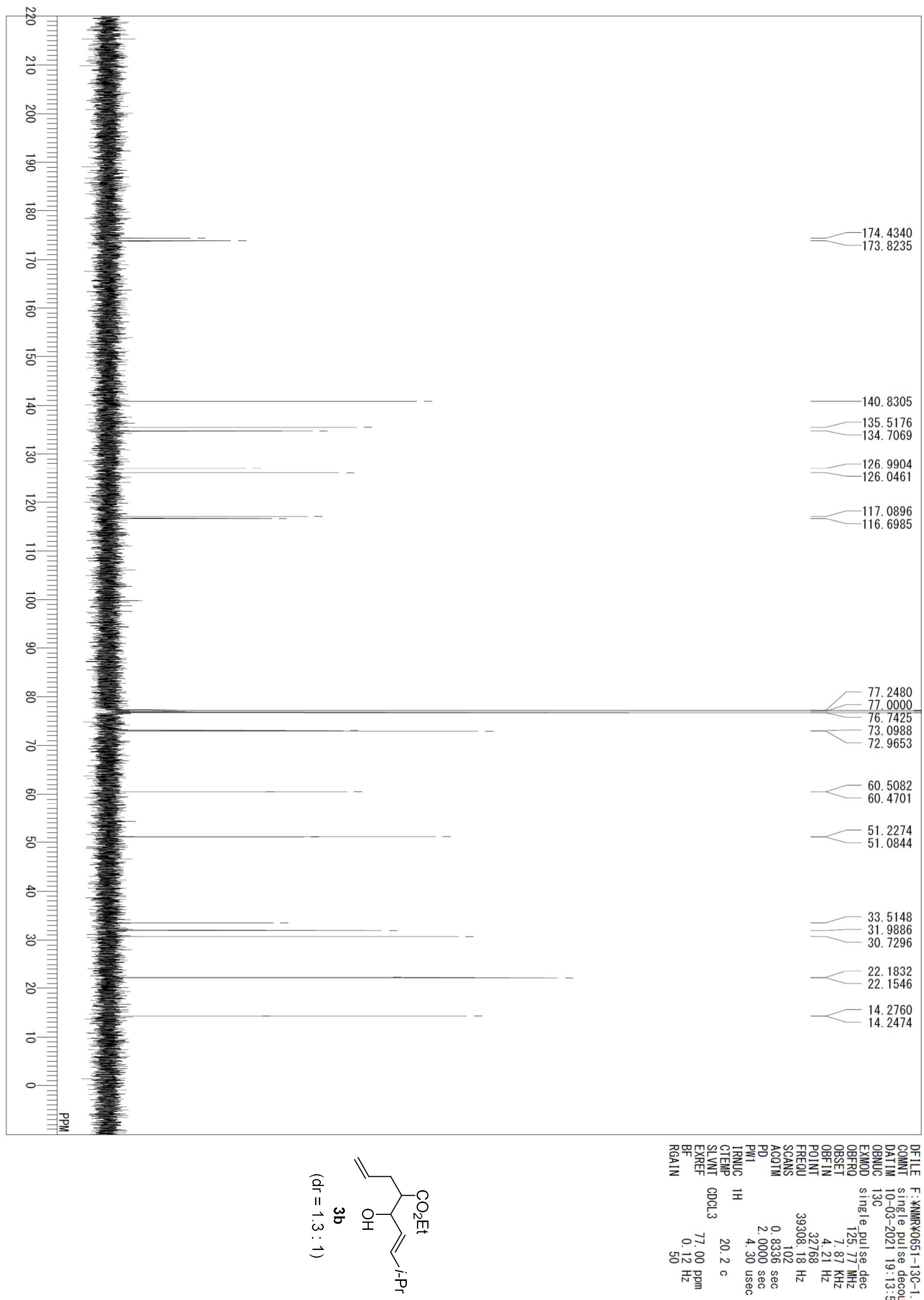

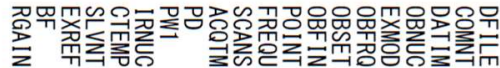

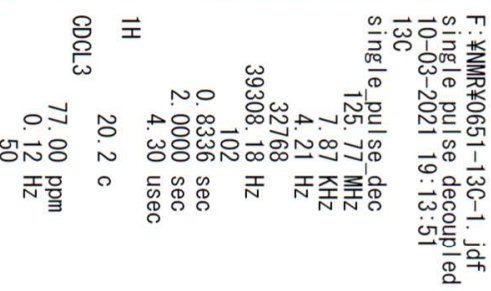




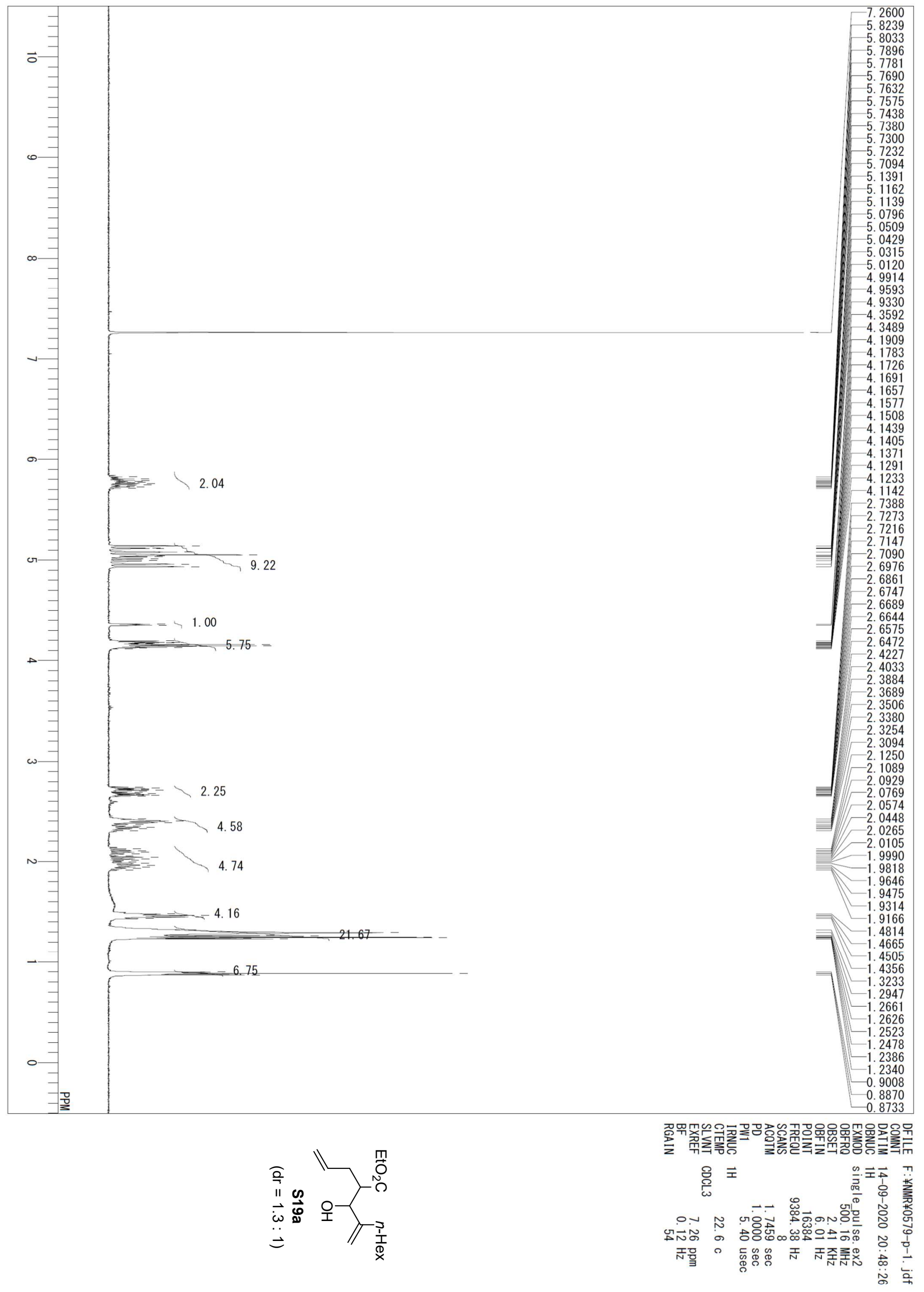



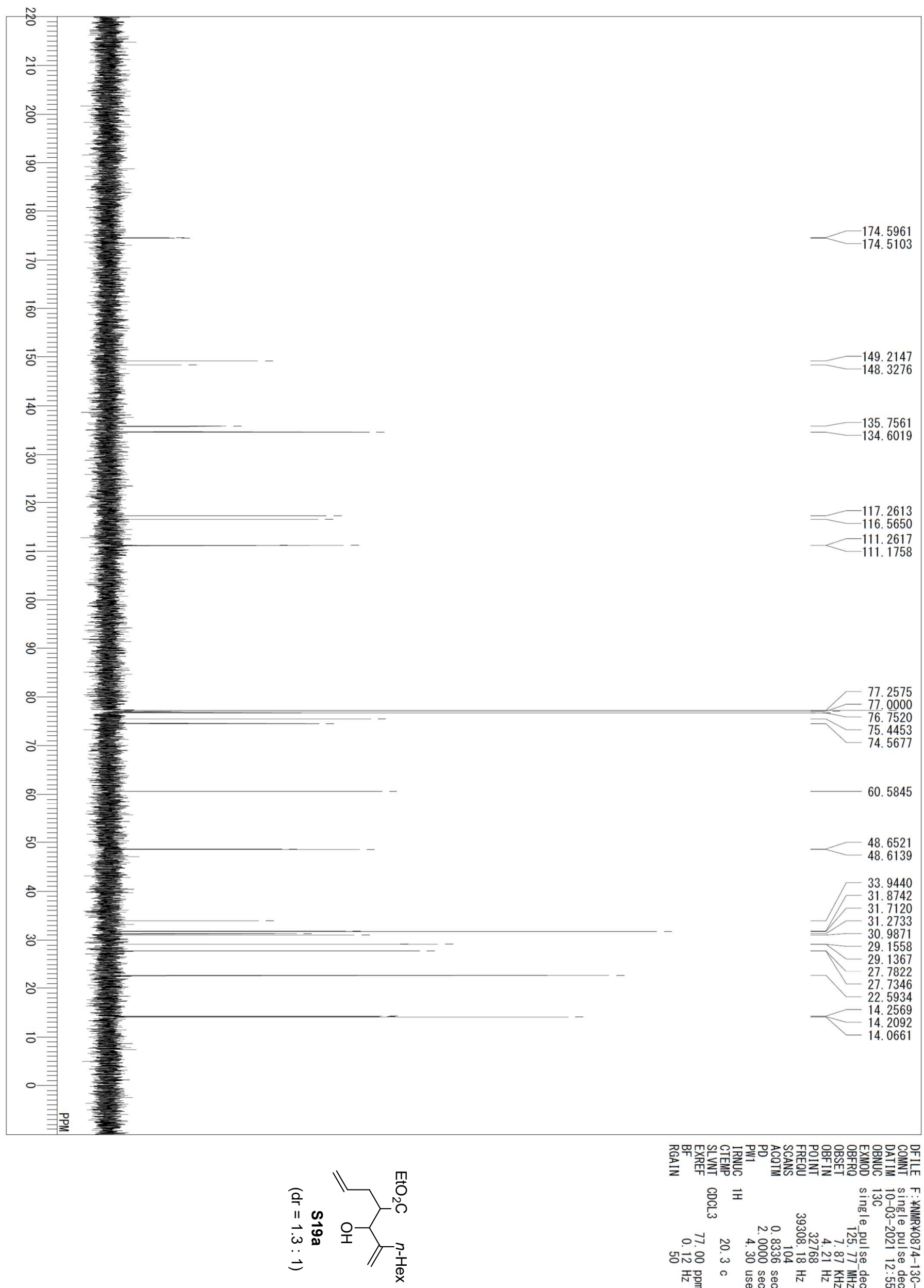

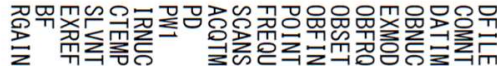

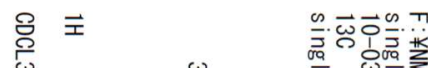

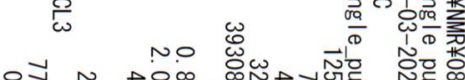
ㄱ․

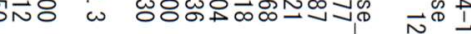

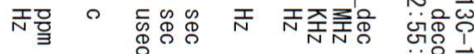
影高视 


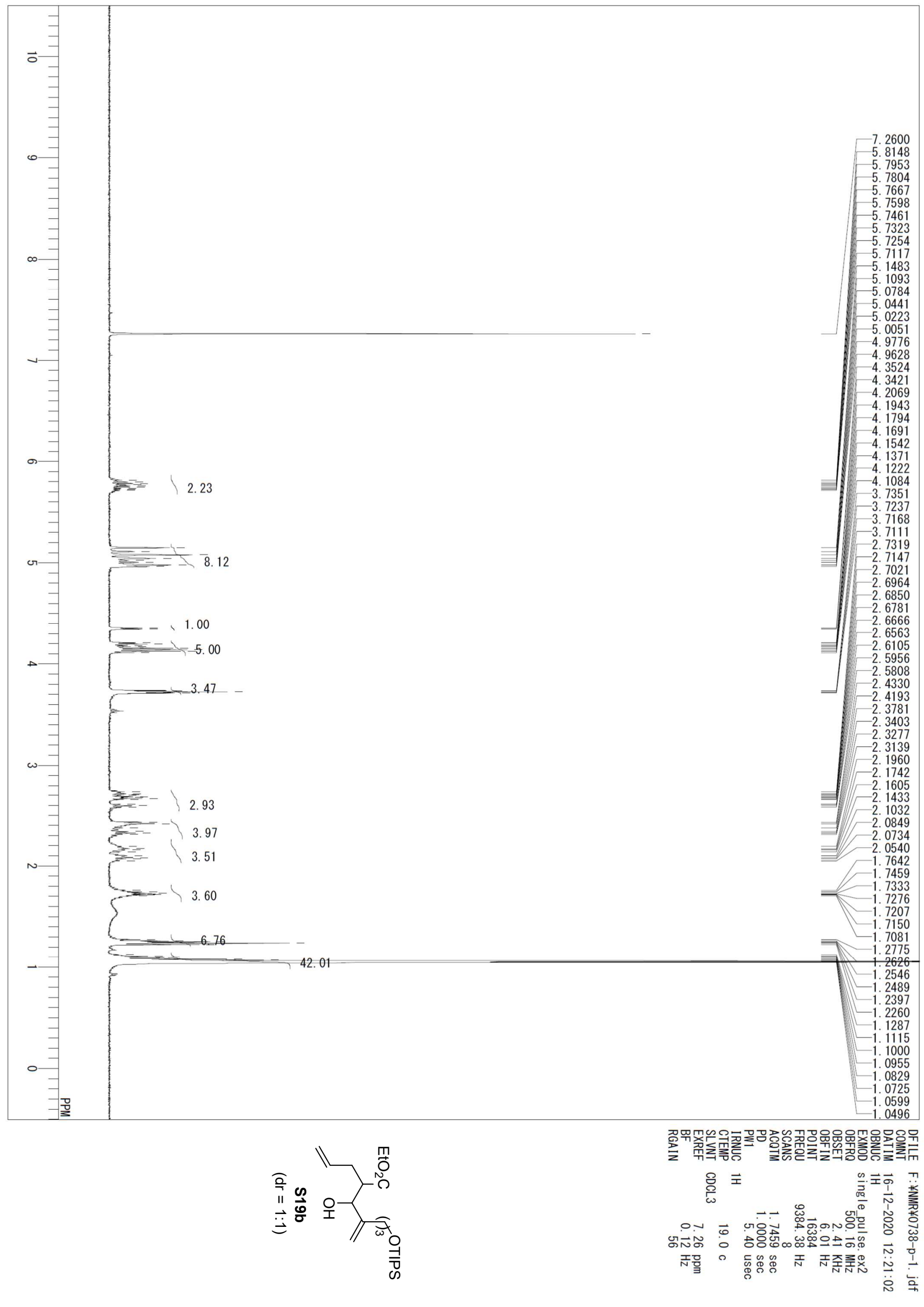




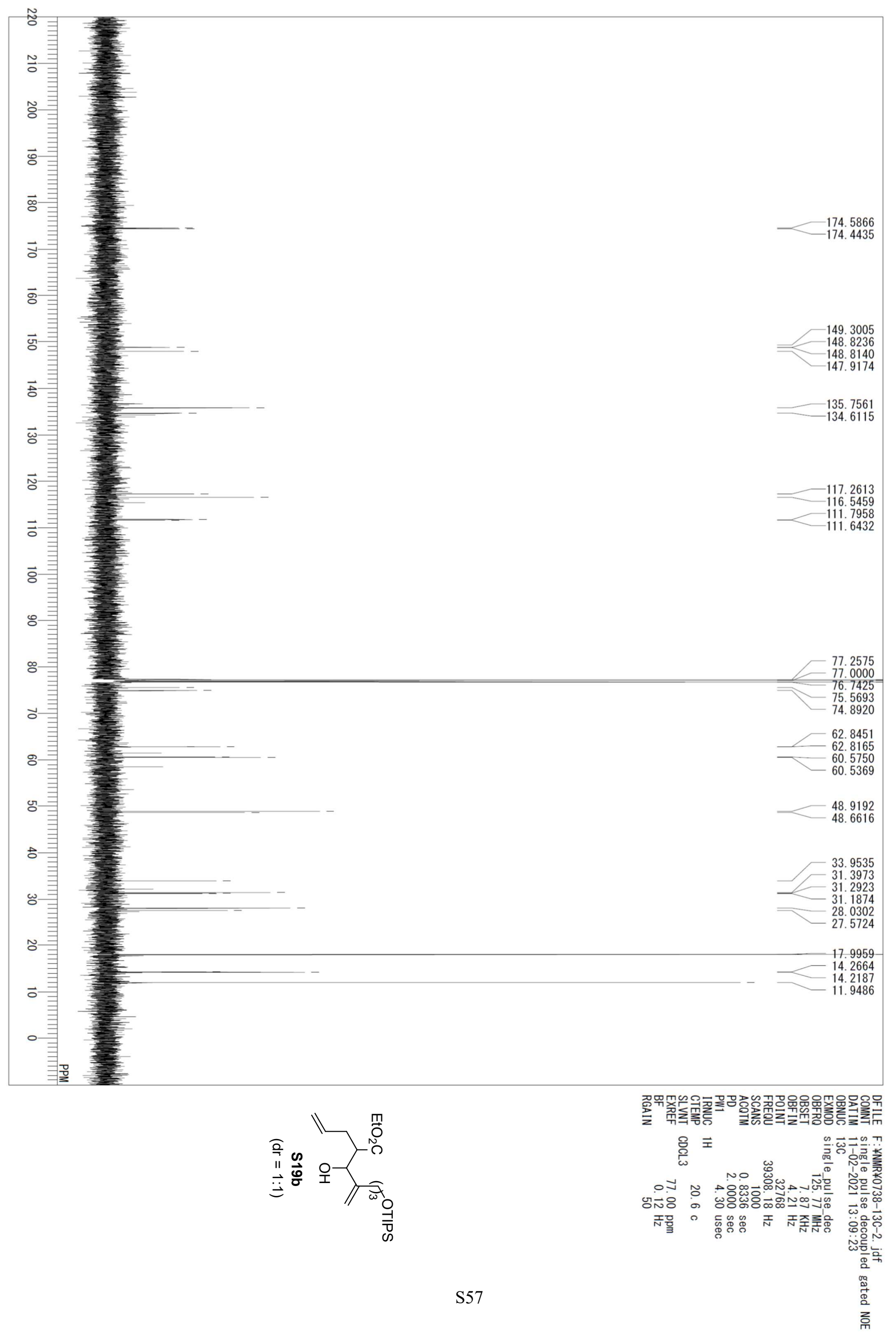




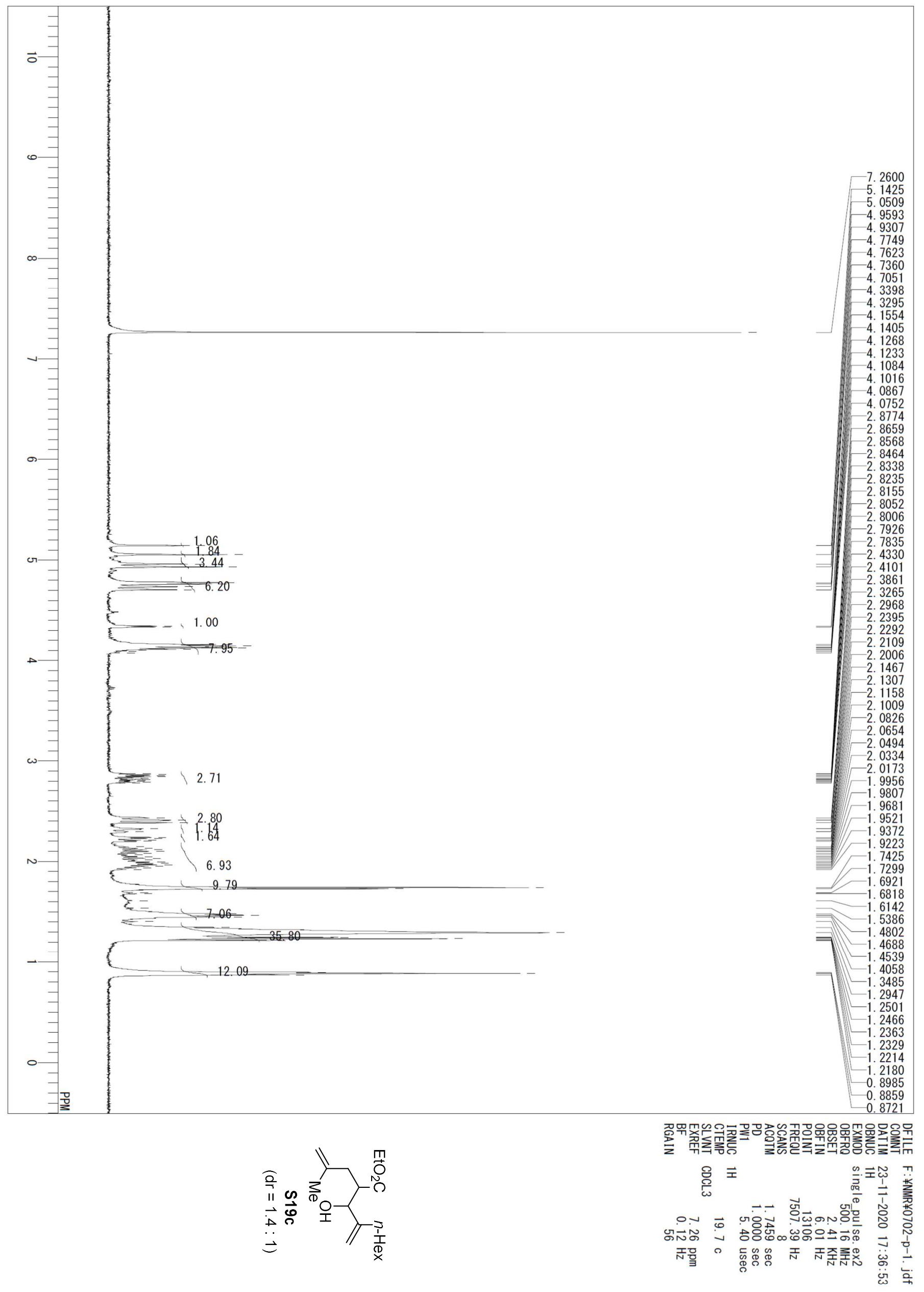



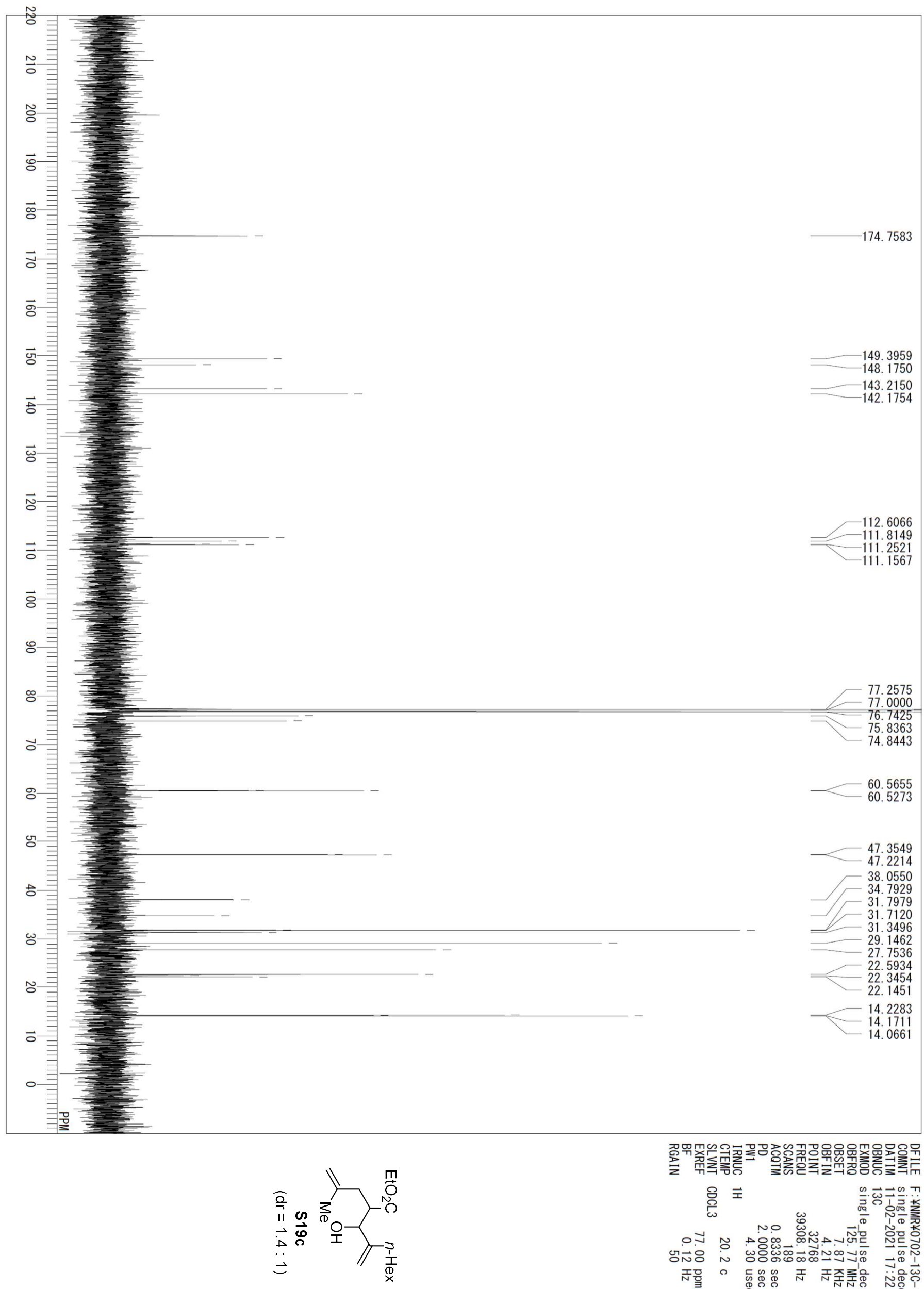

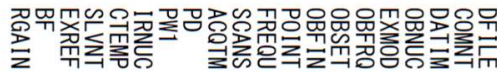

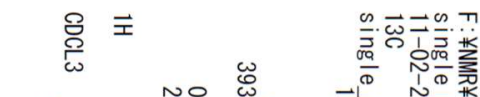

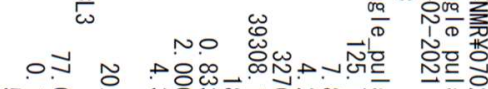

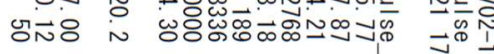

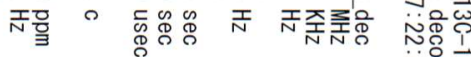




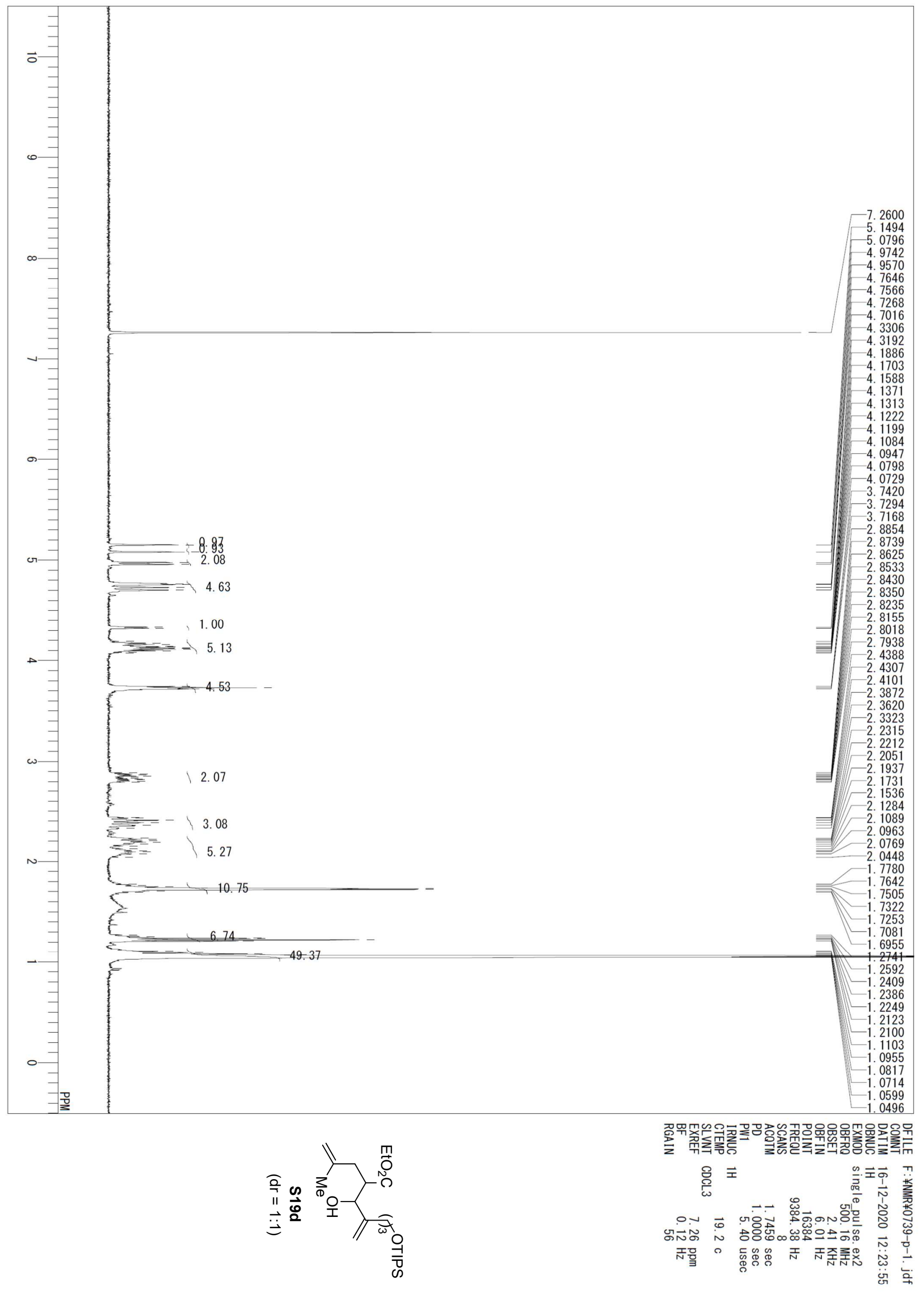




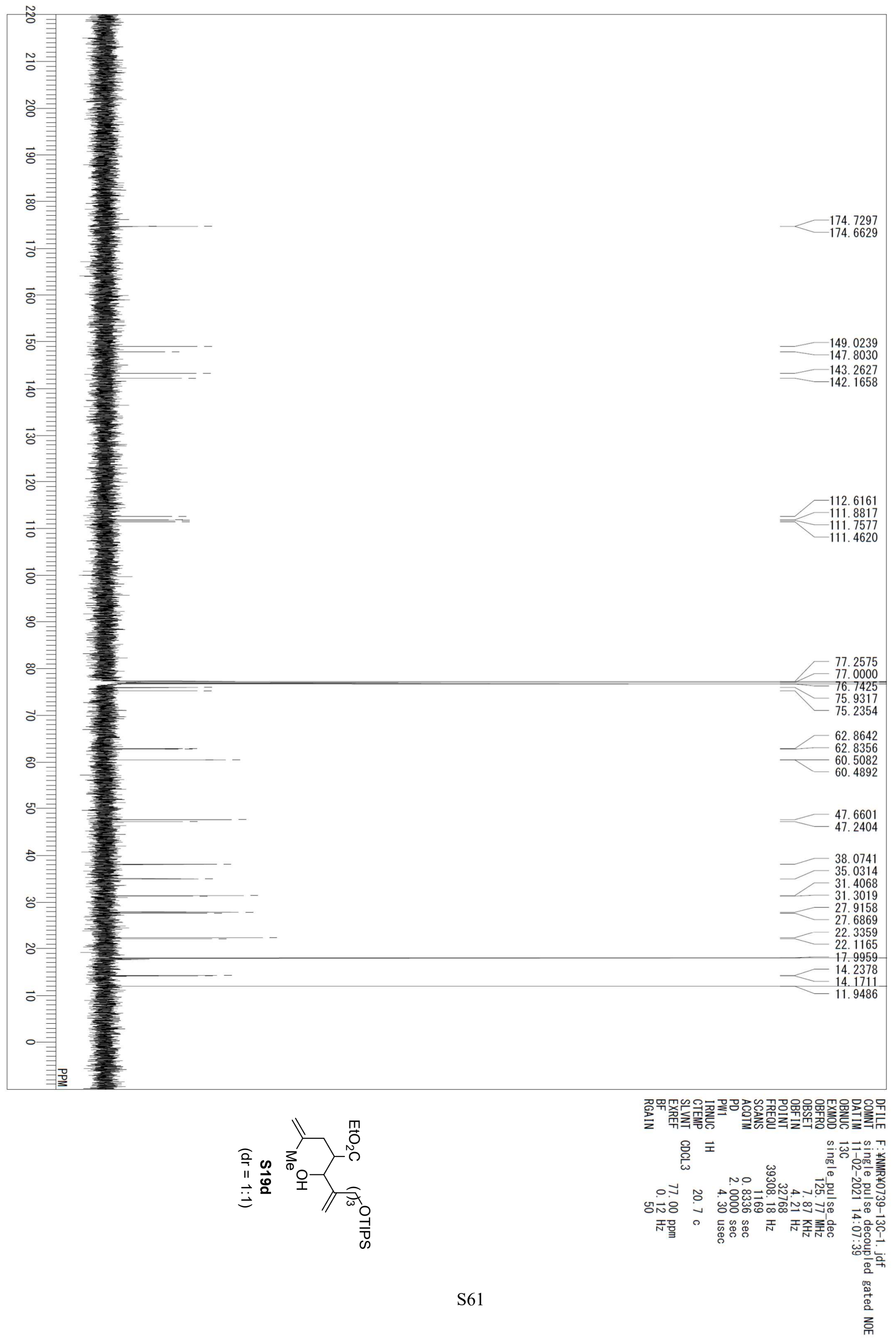




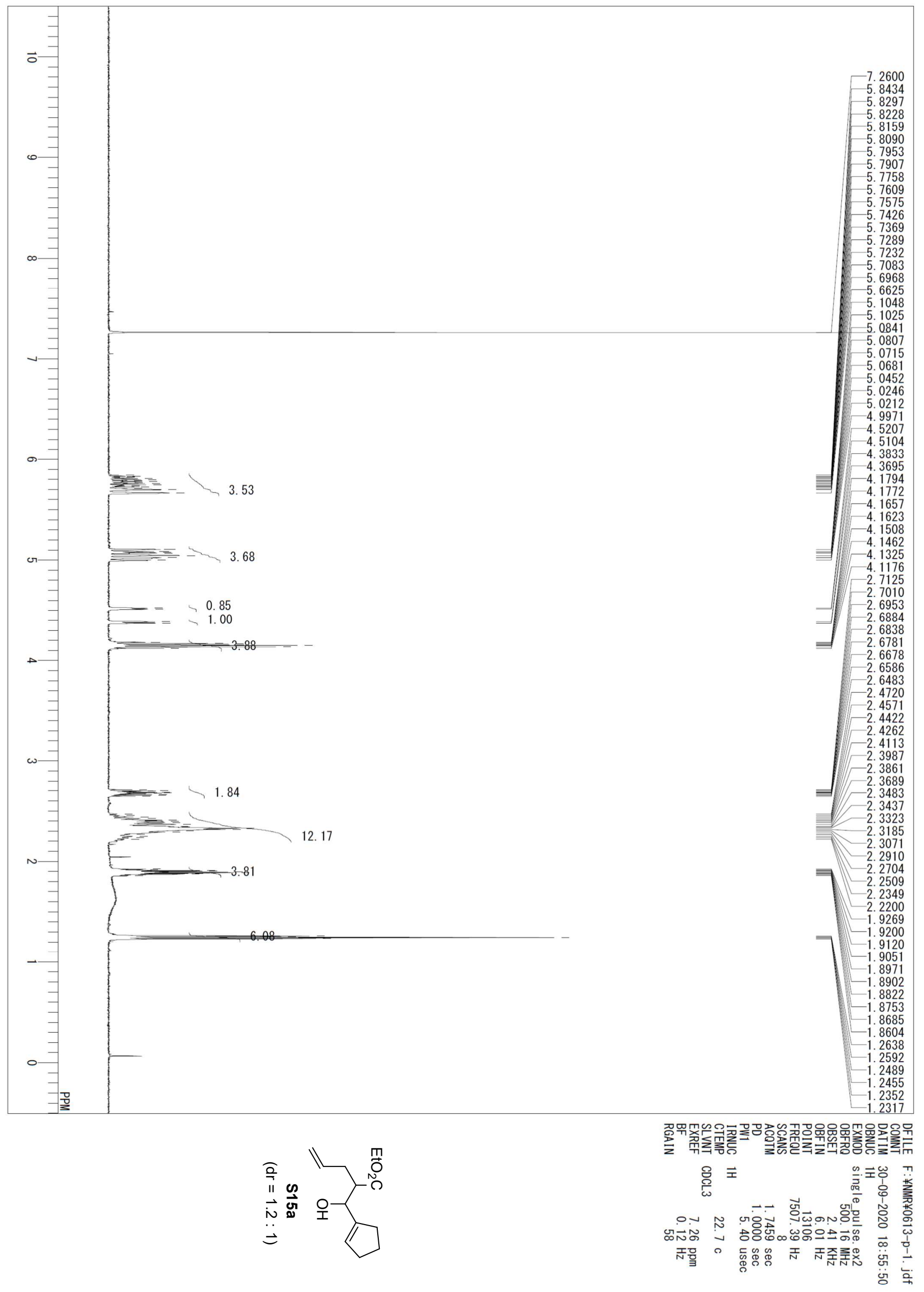



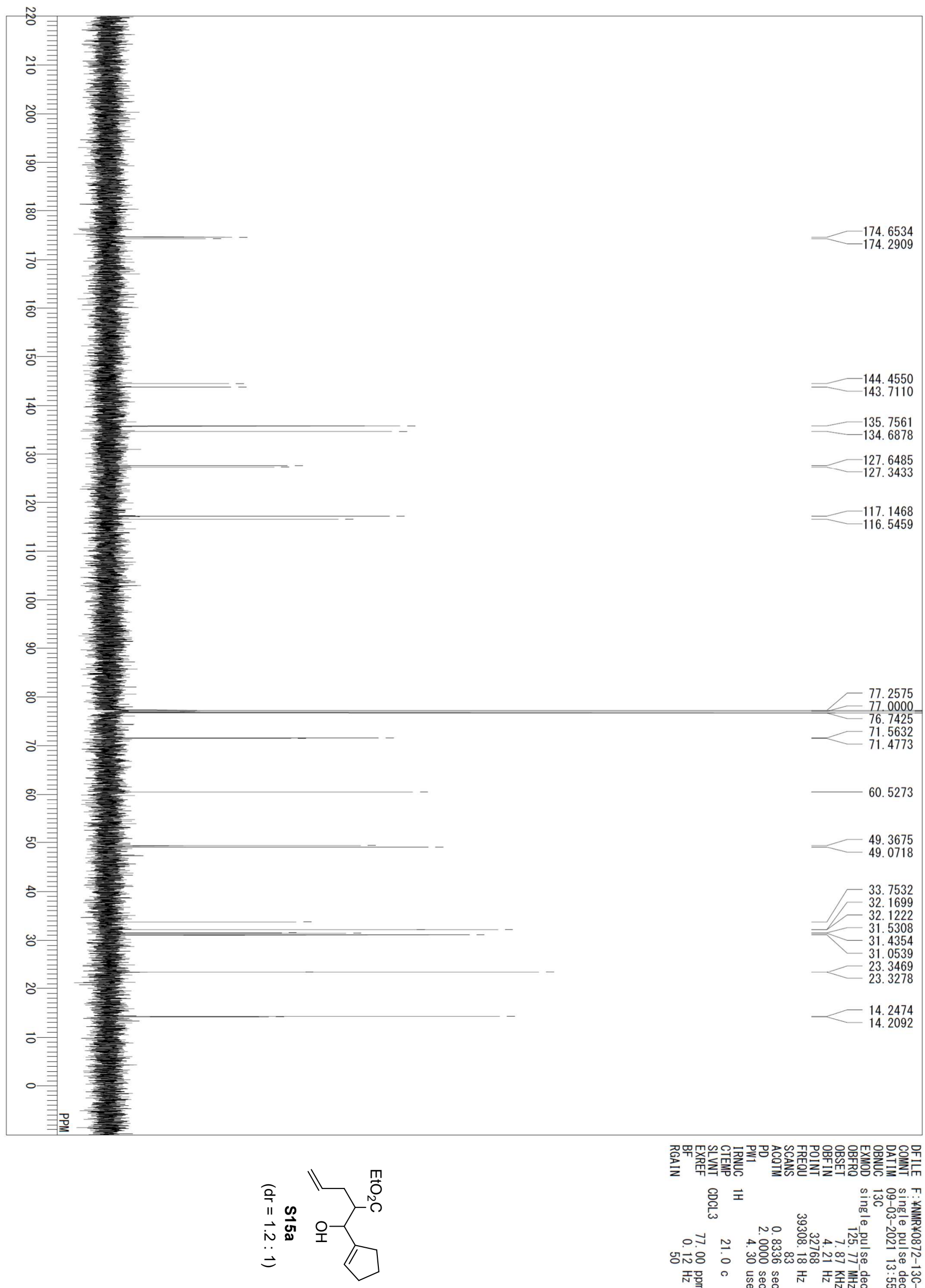

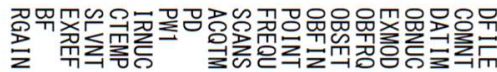

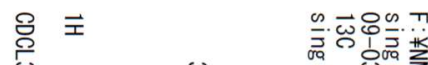

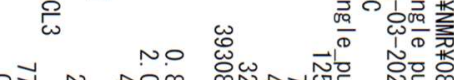

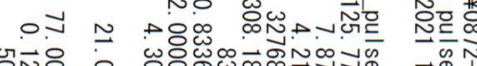
西

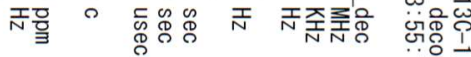

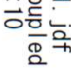




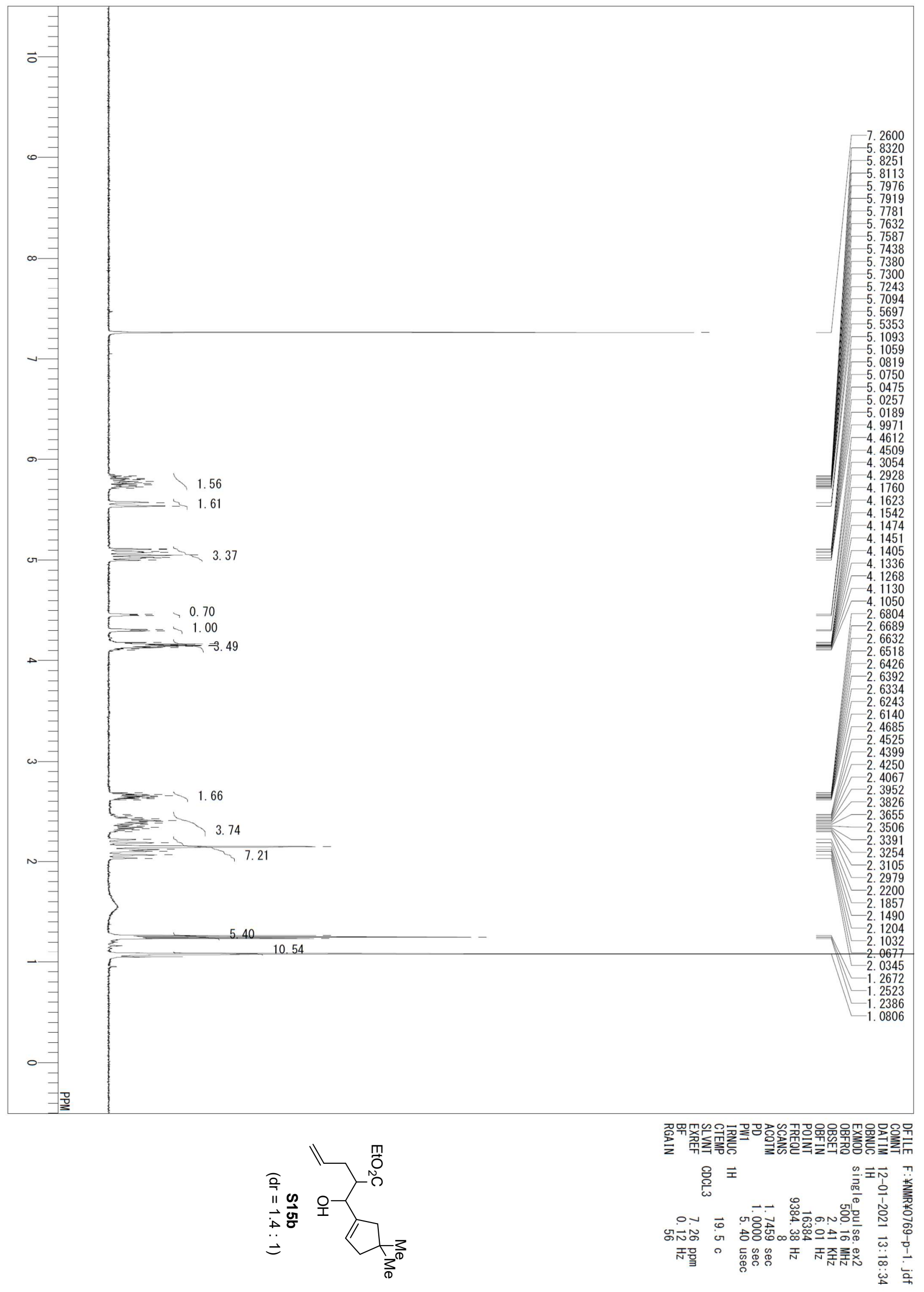



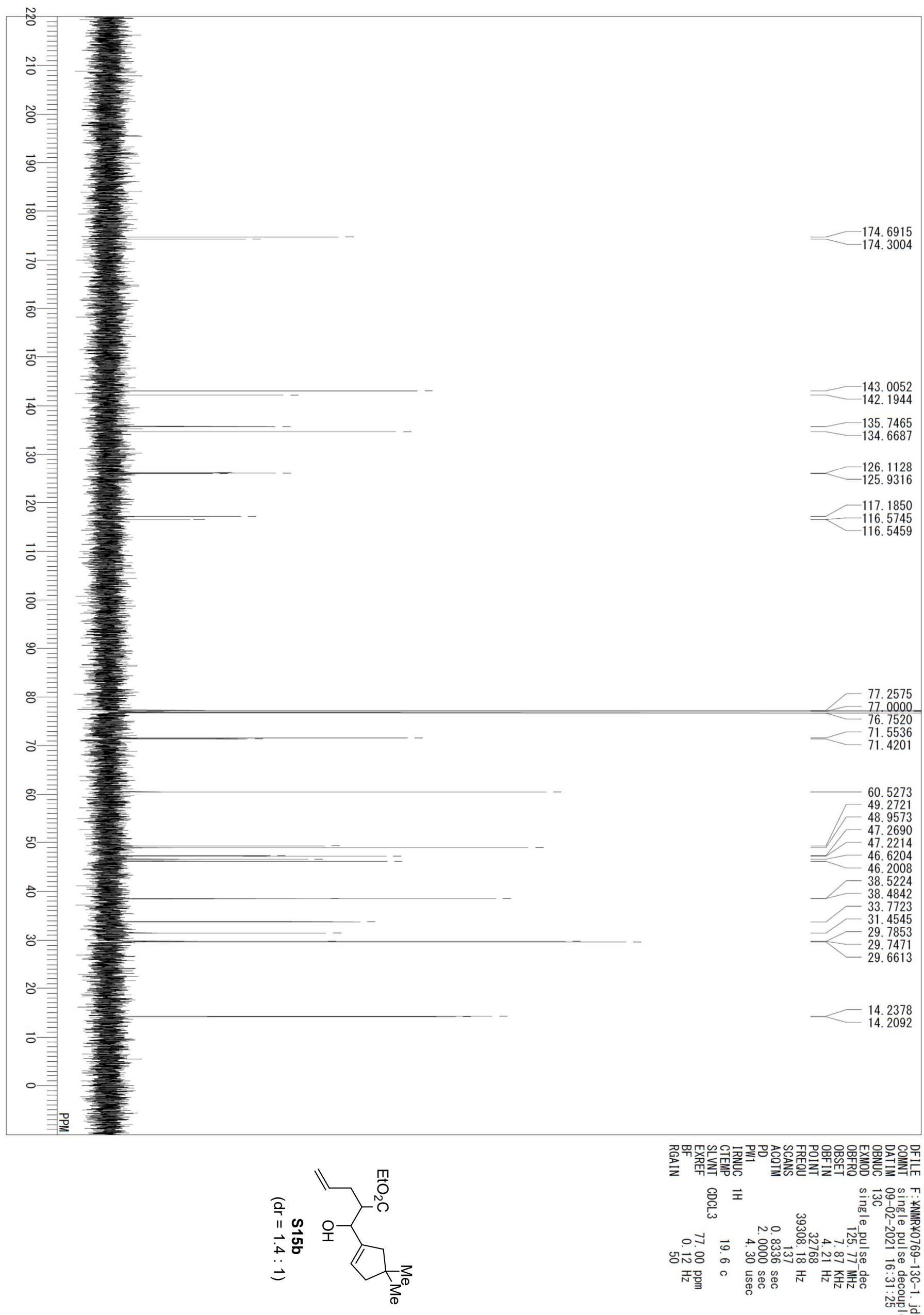

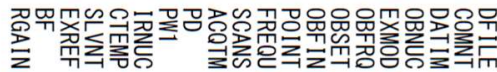

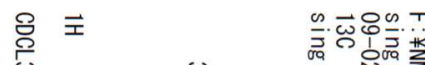

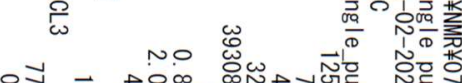
유요 की

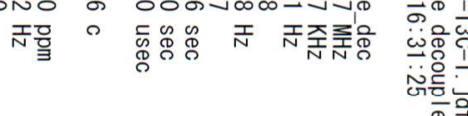




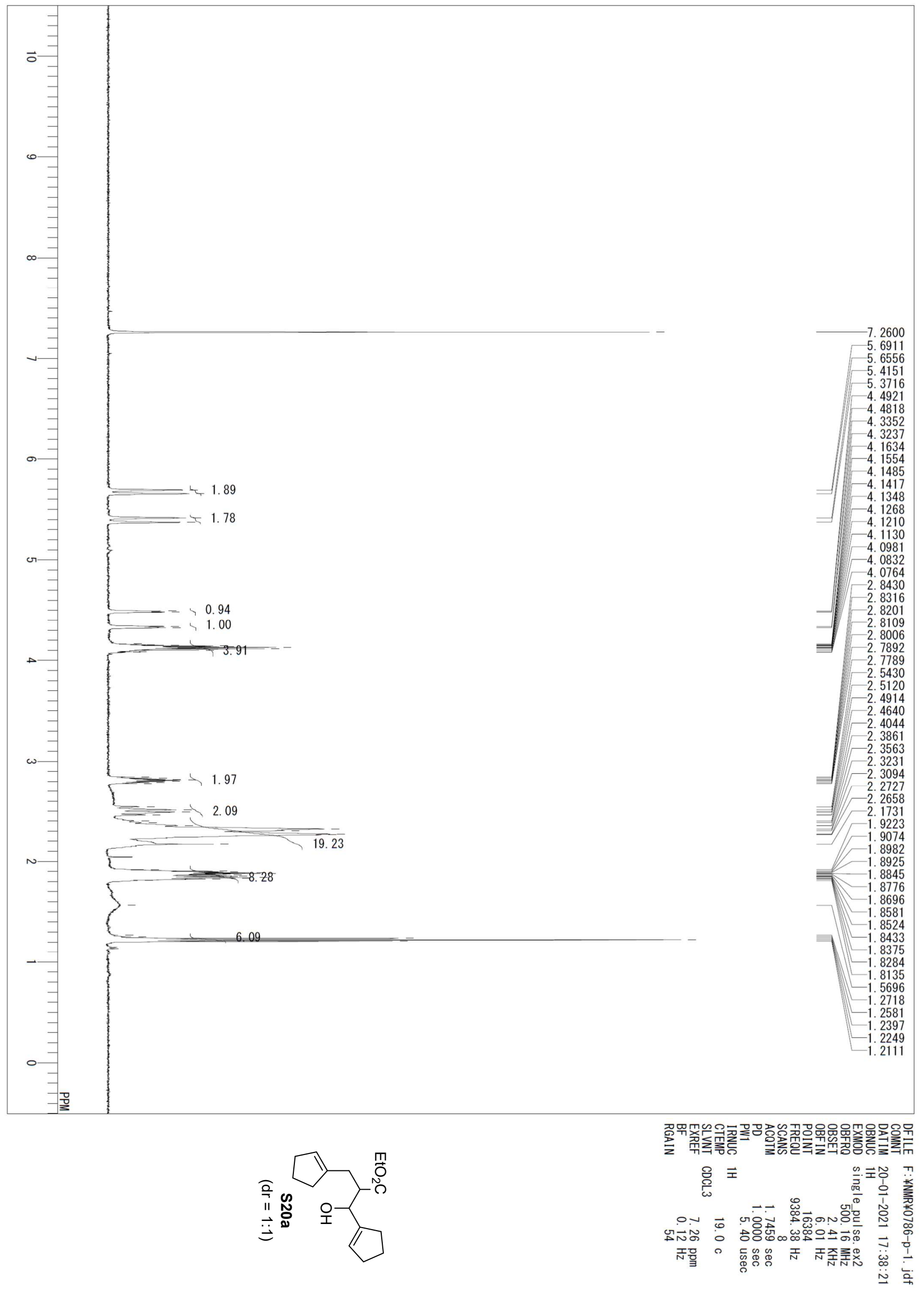




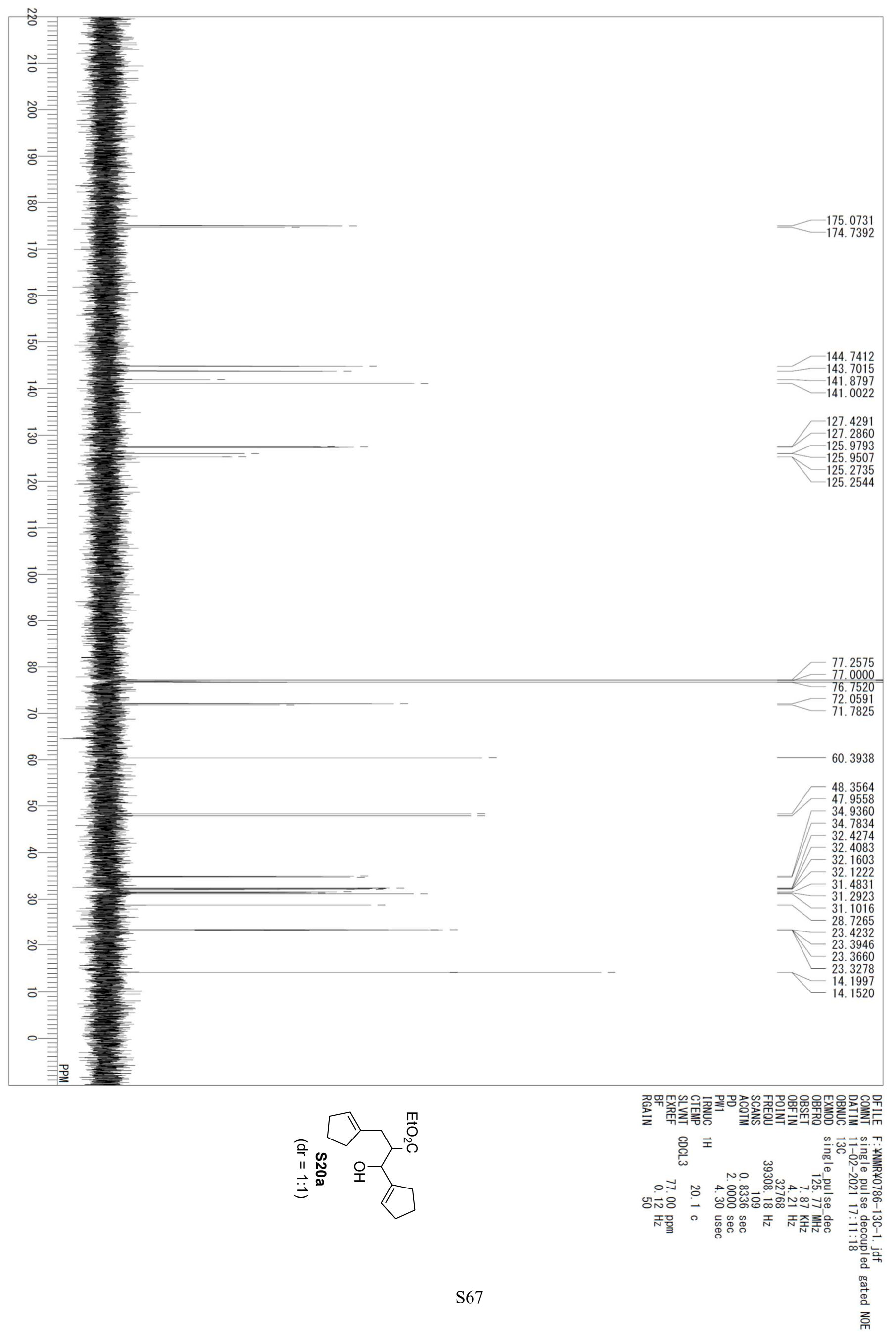



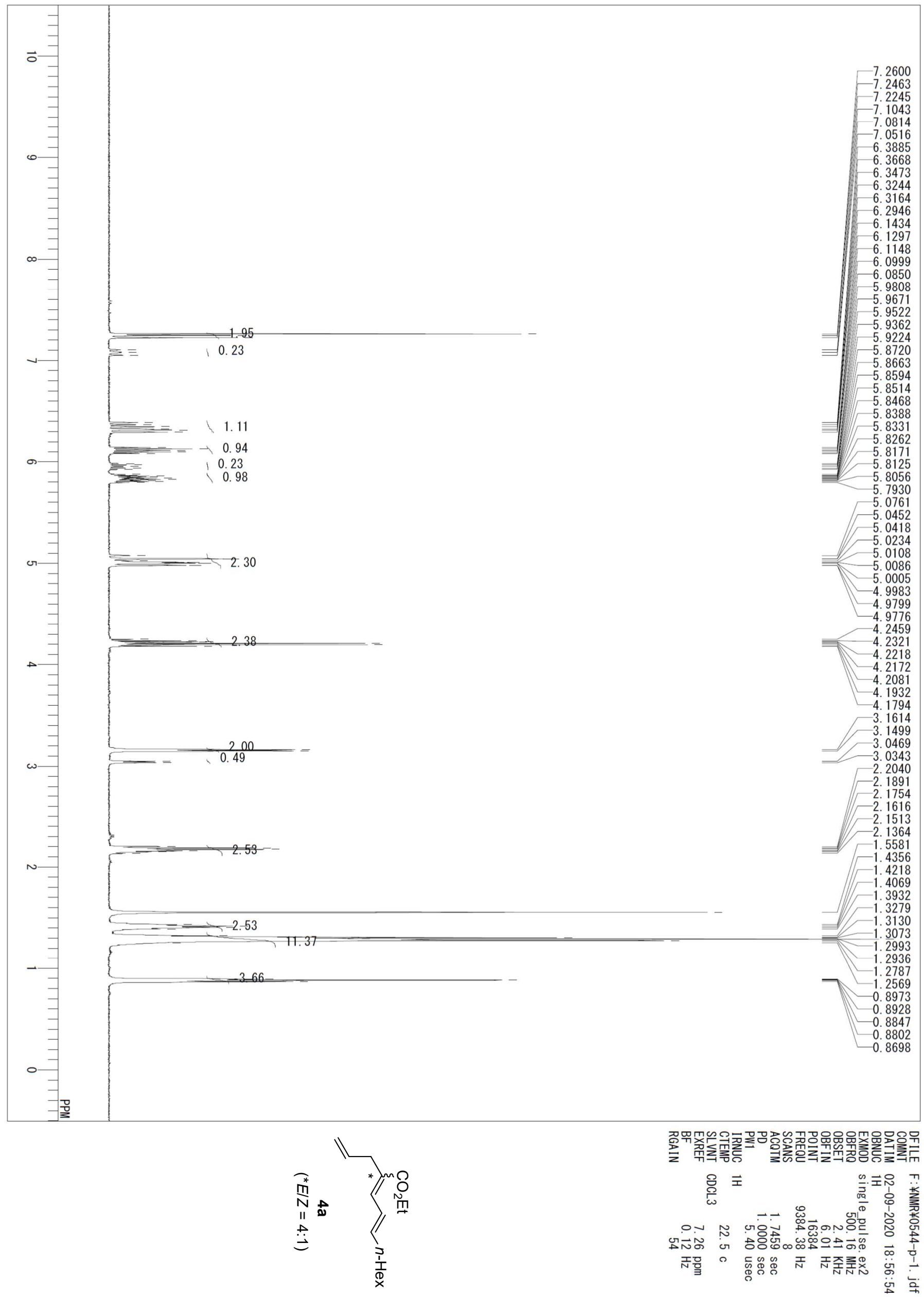

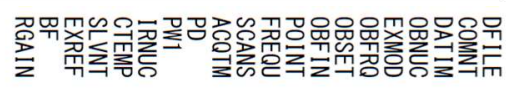

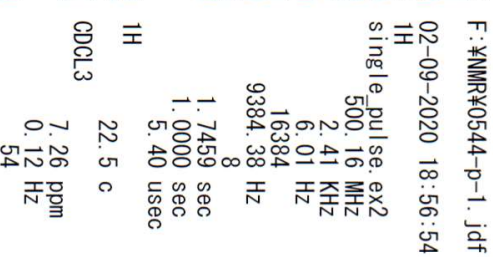




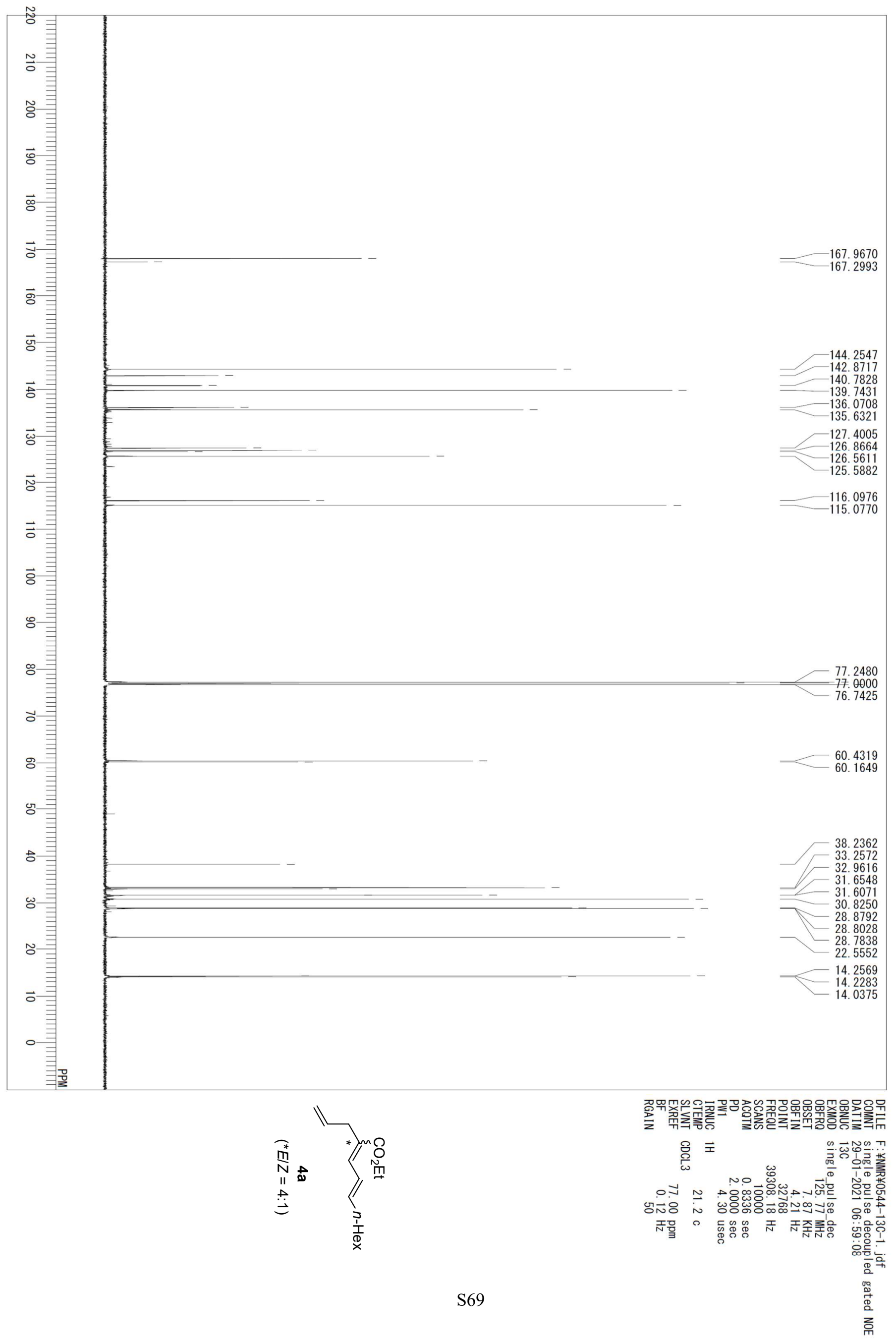



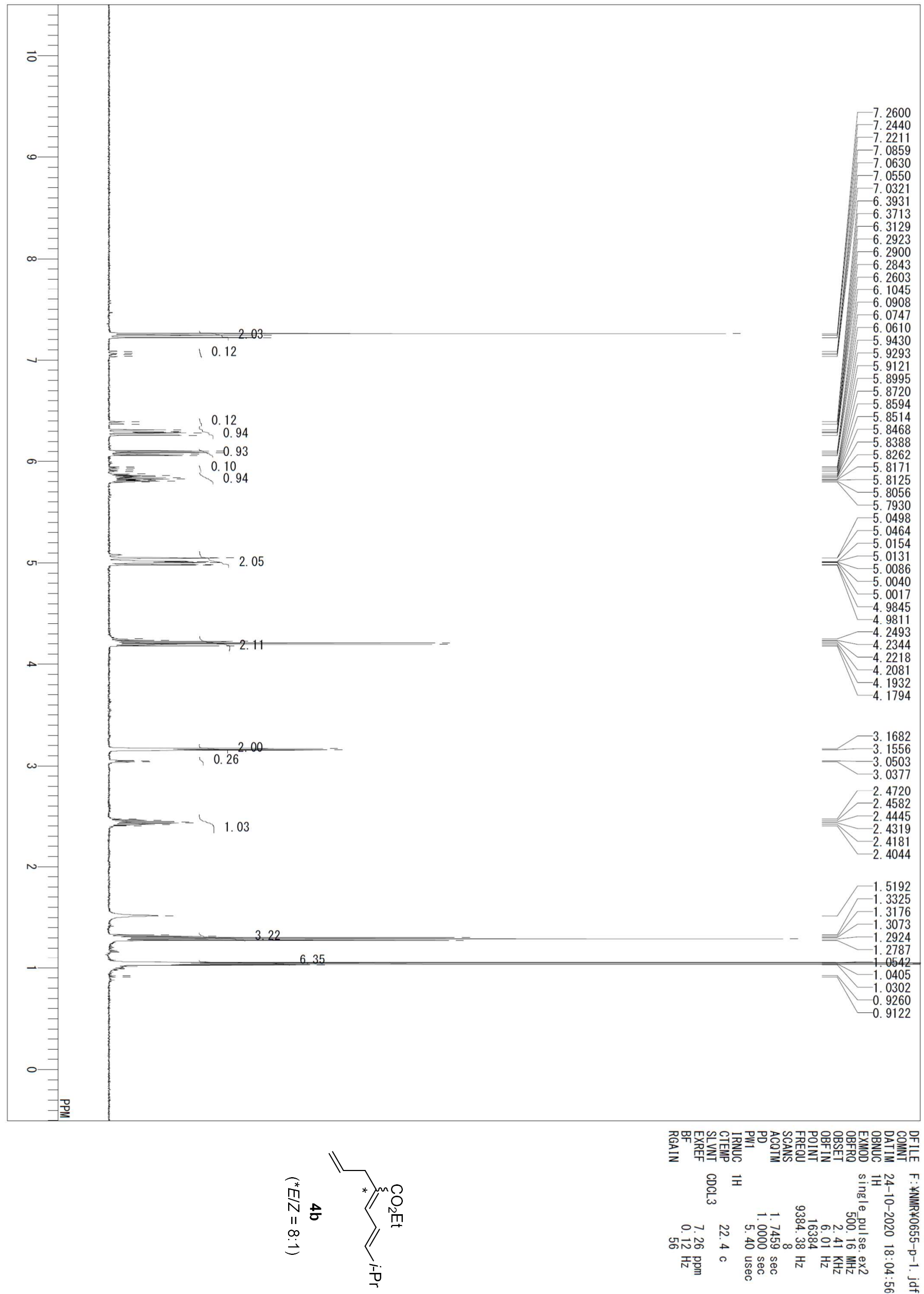

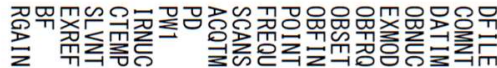

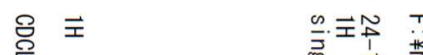

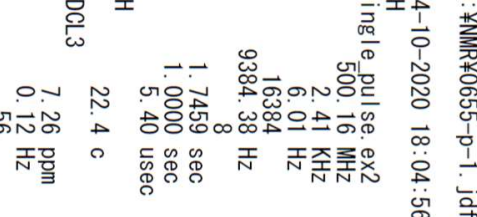




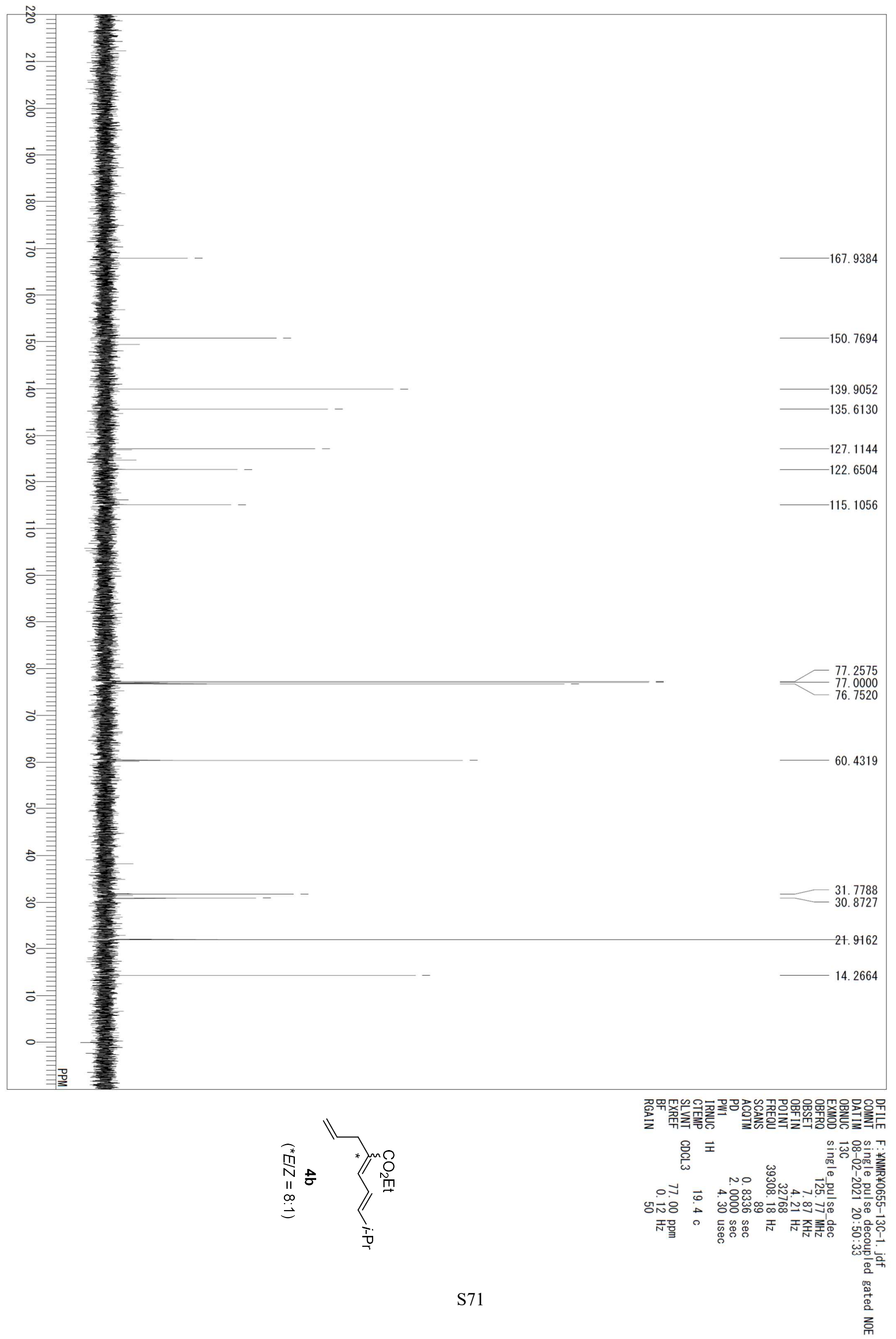



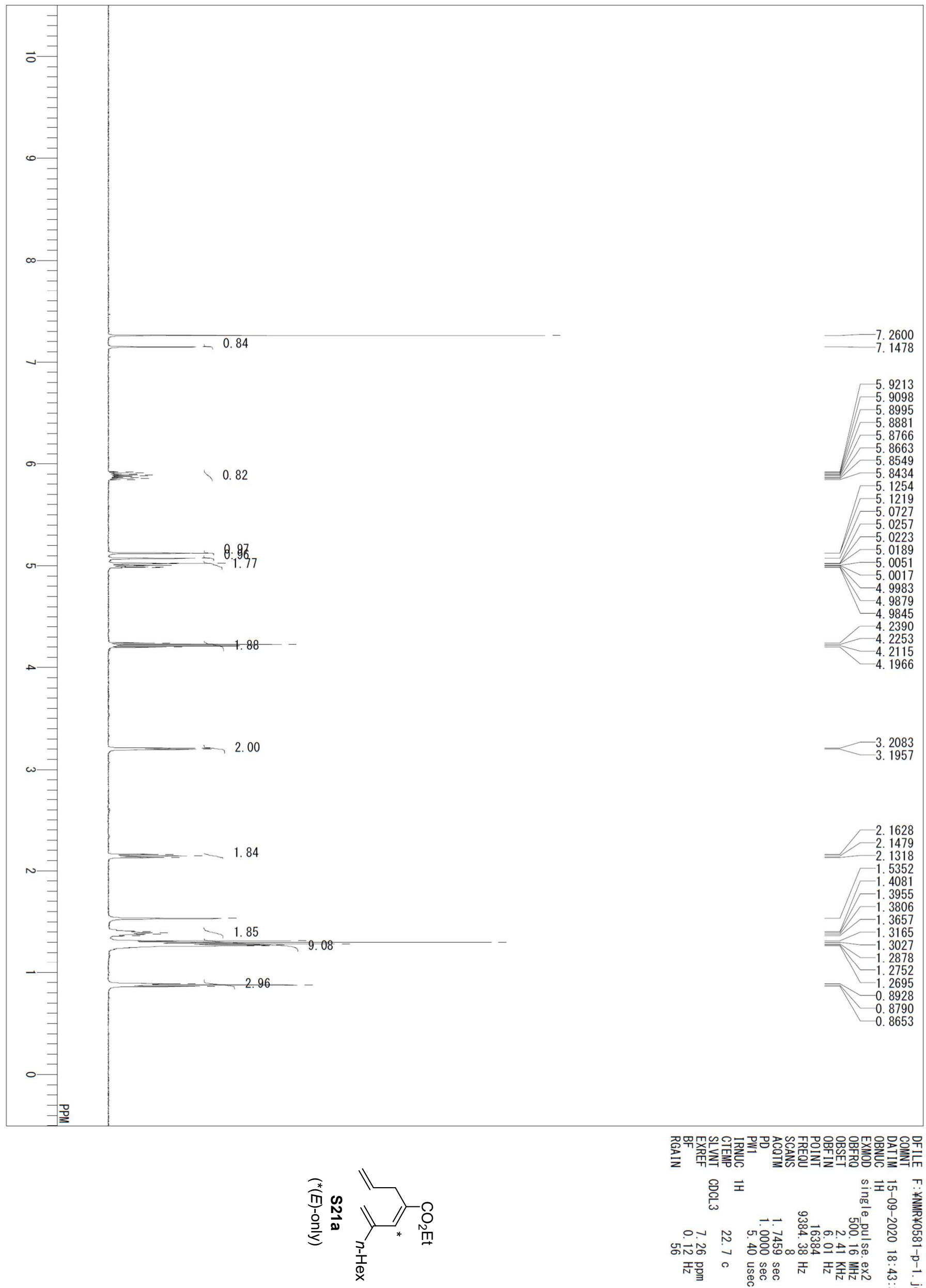

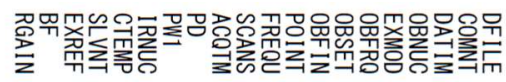

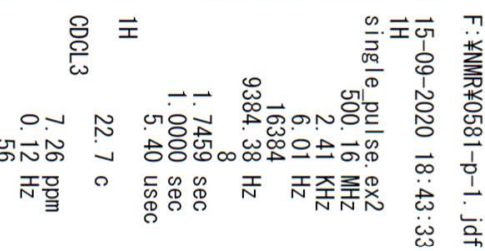



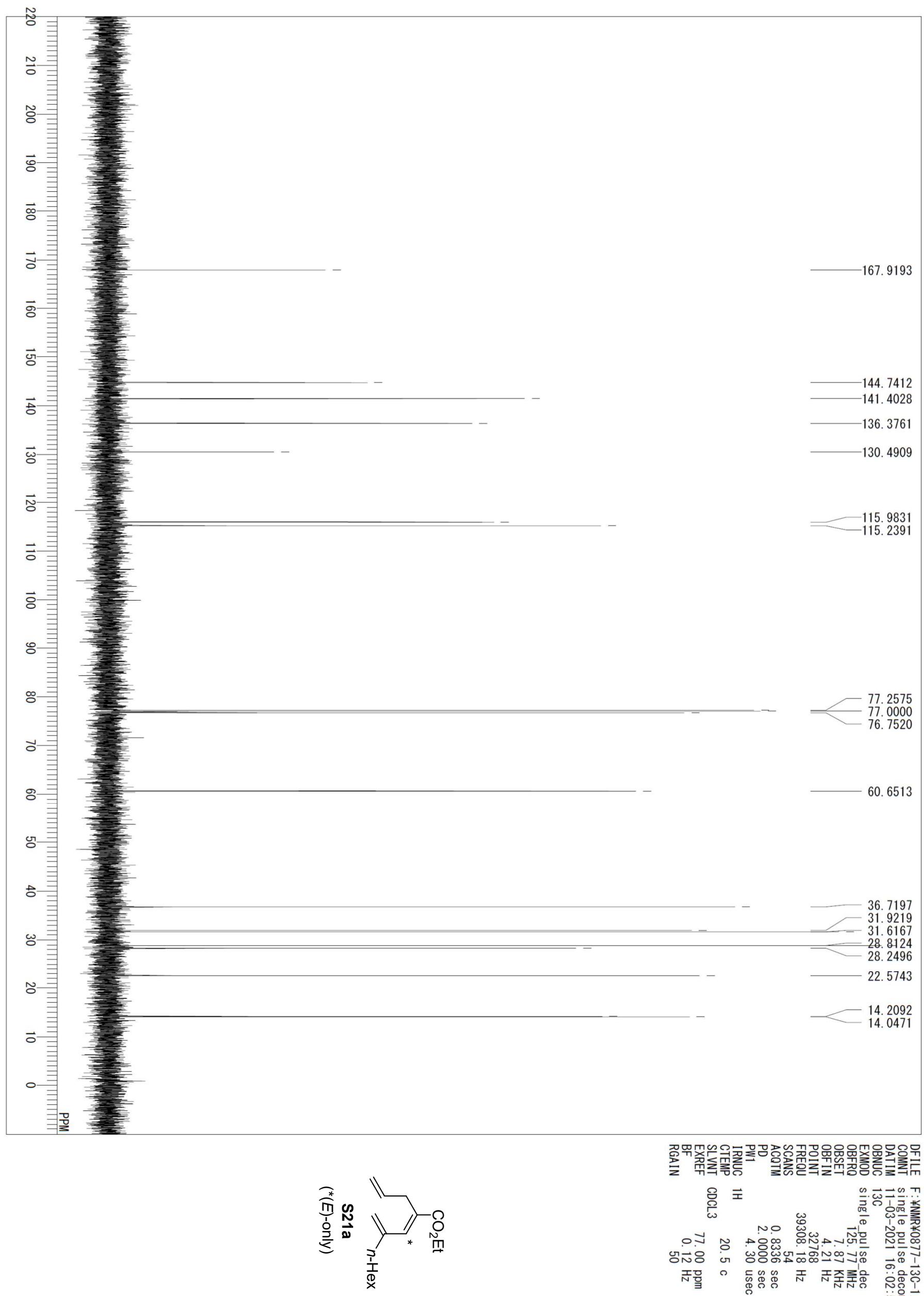

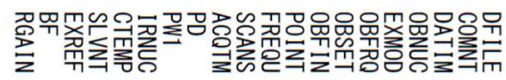

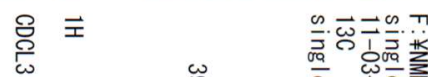

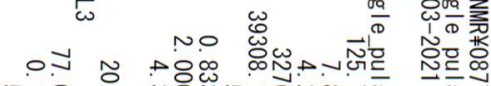

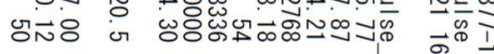

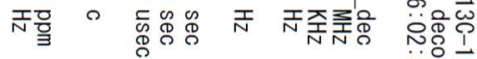



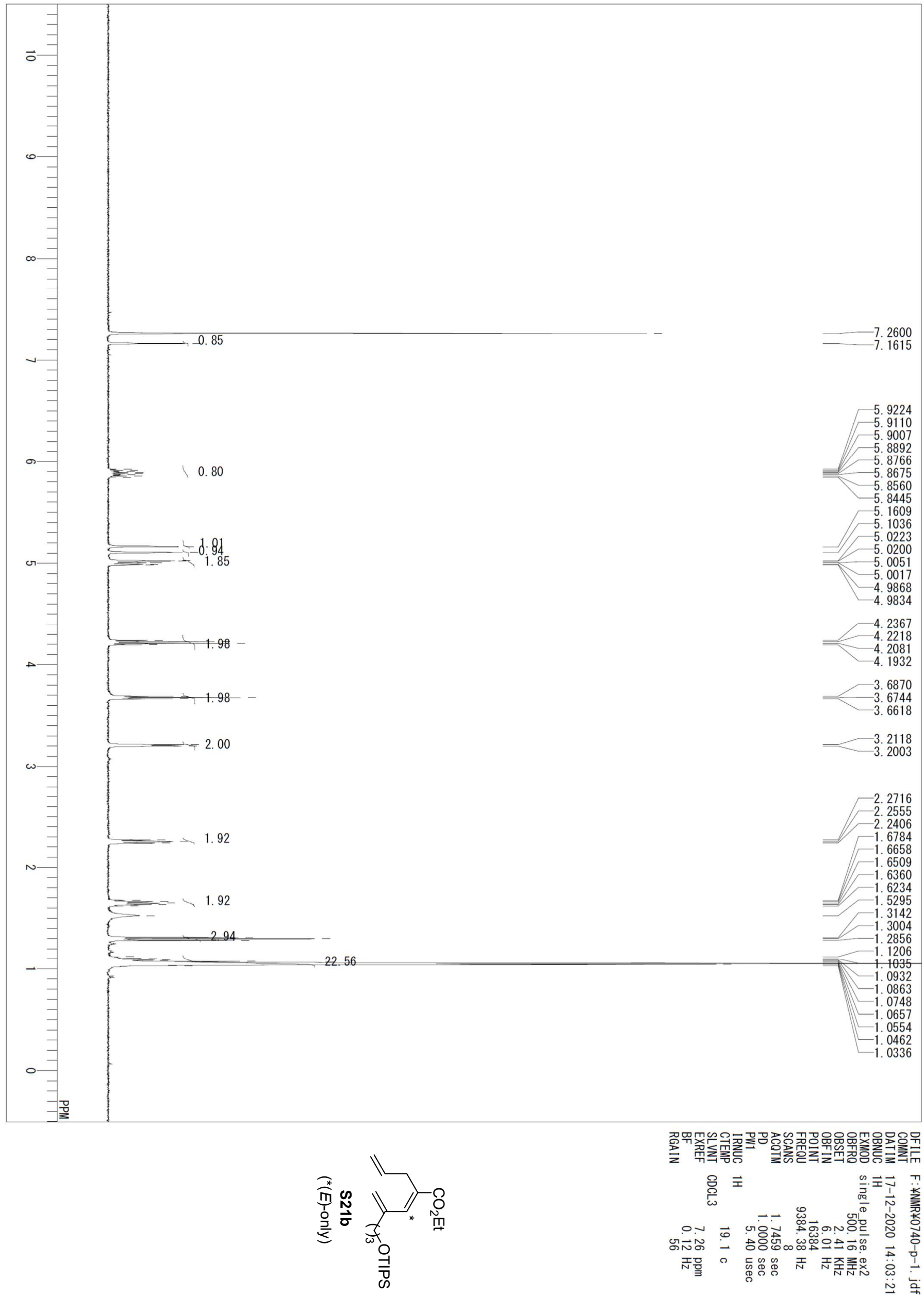

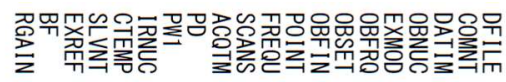

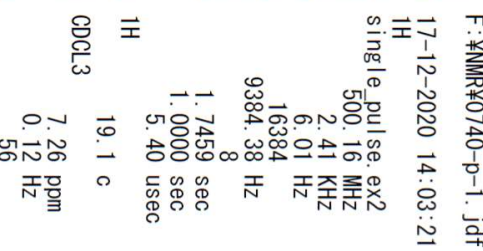




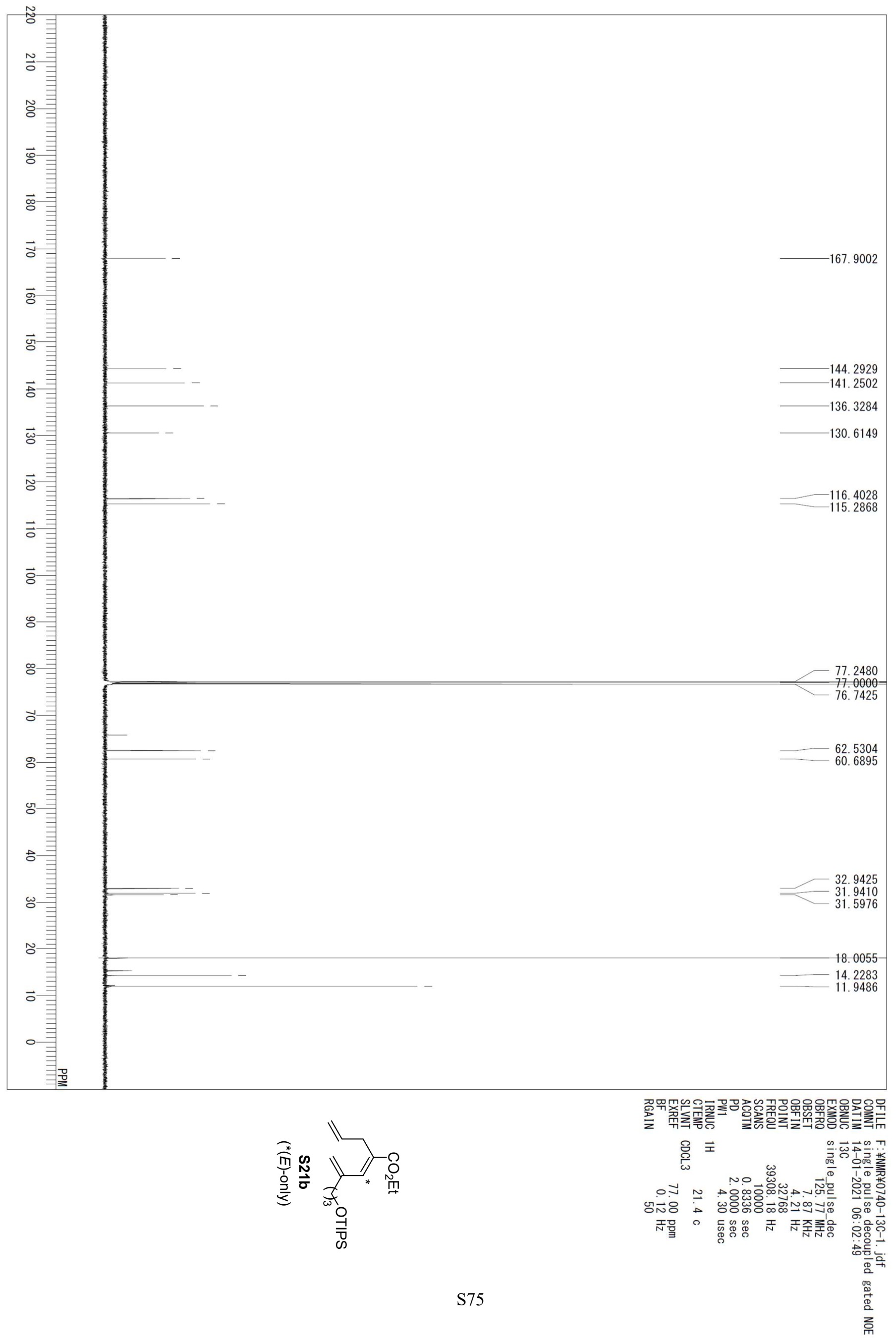



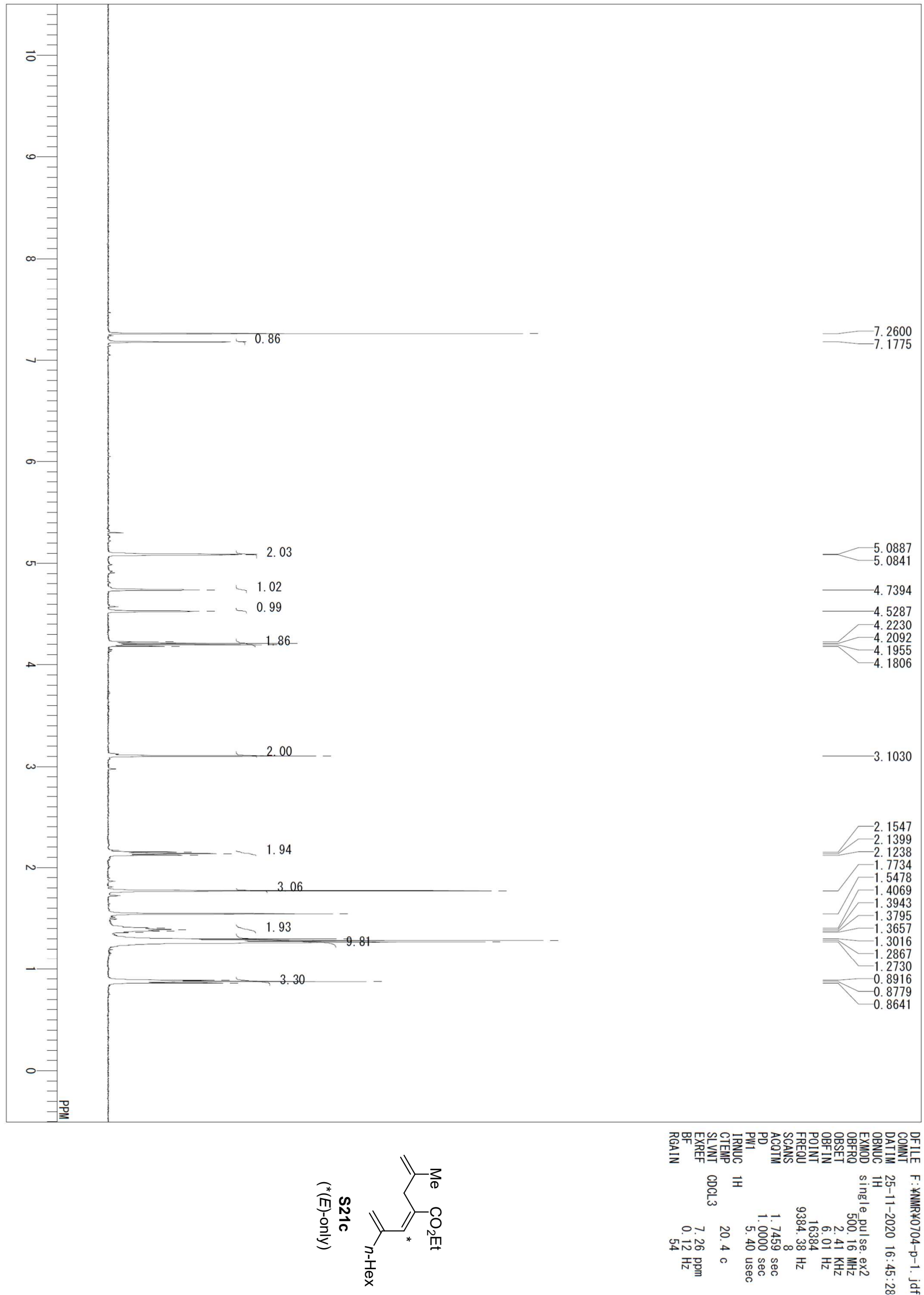

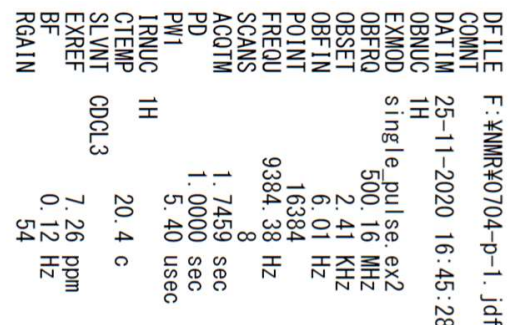



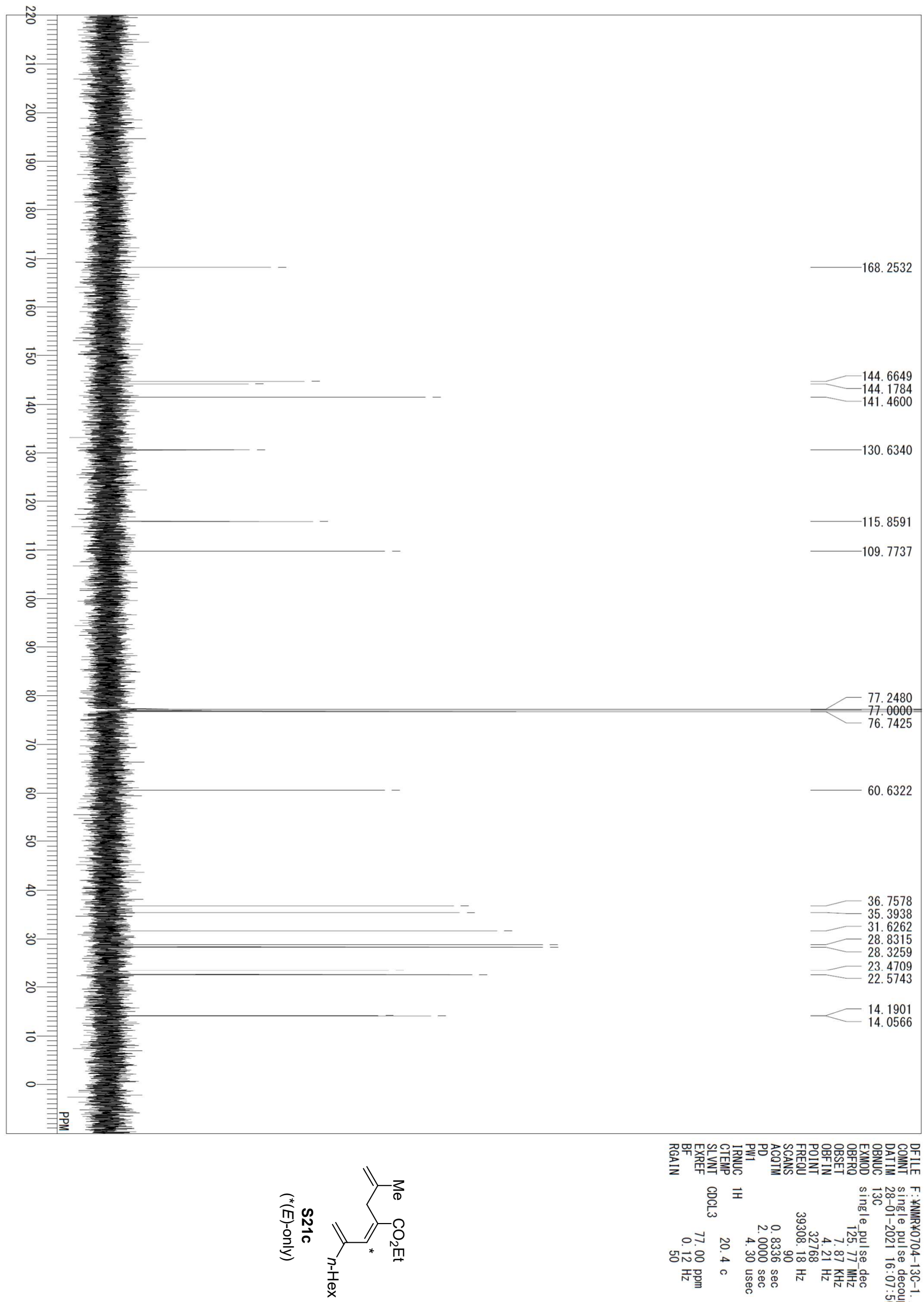

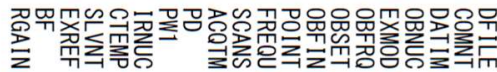

总 $\overline{2}$. O

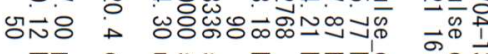

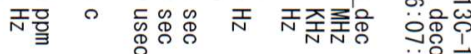

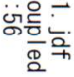



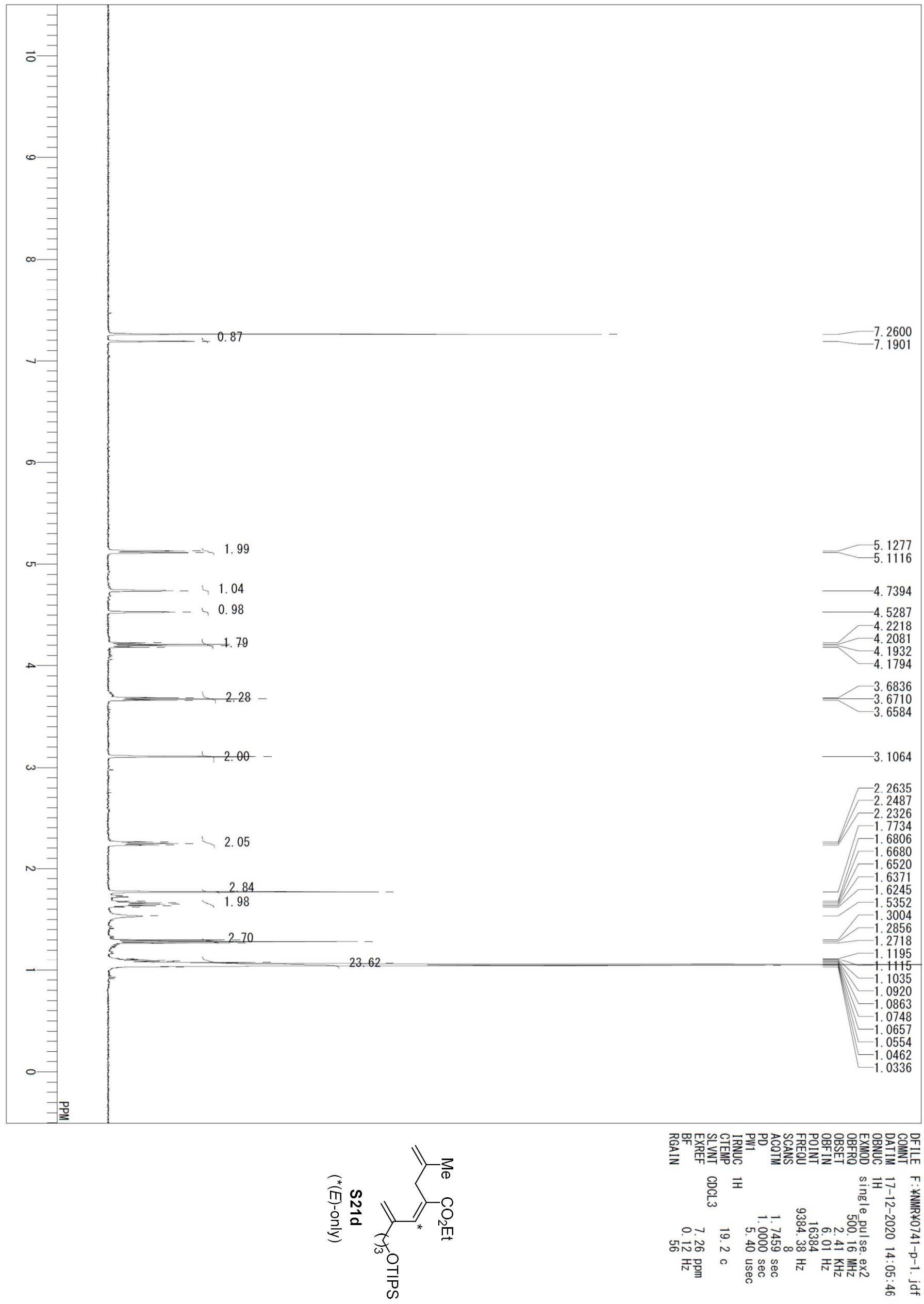

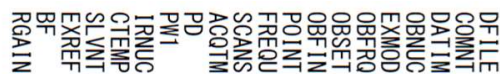

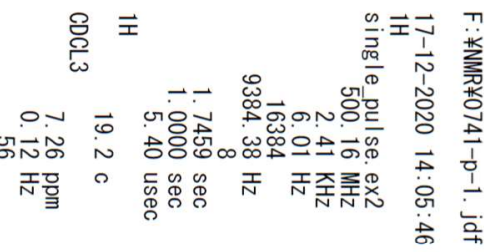




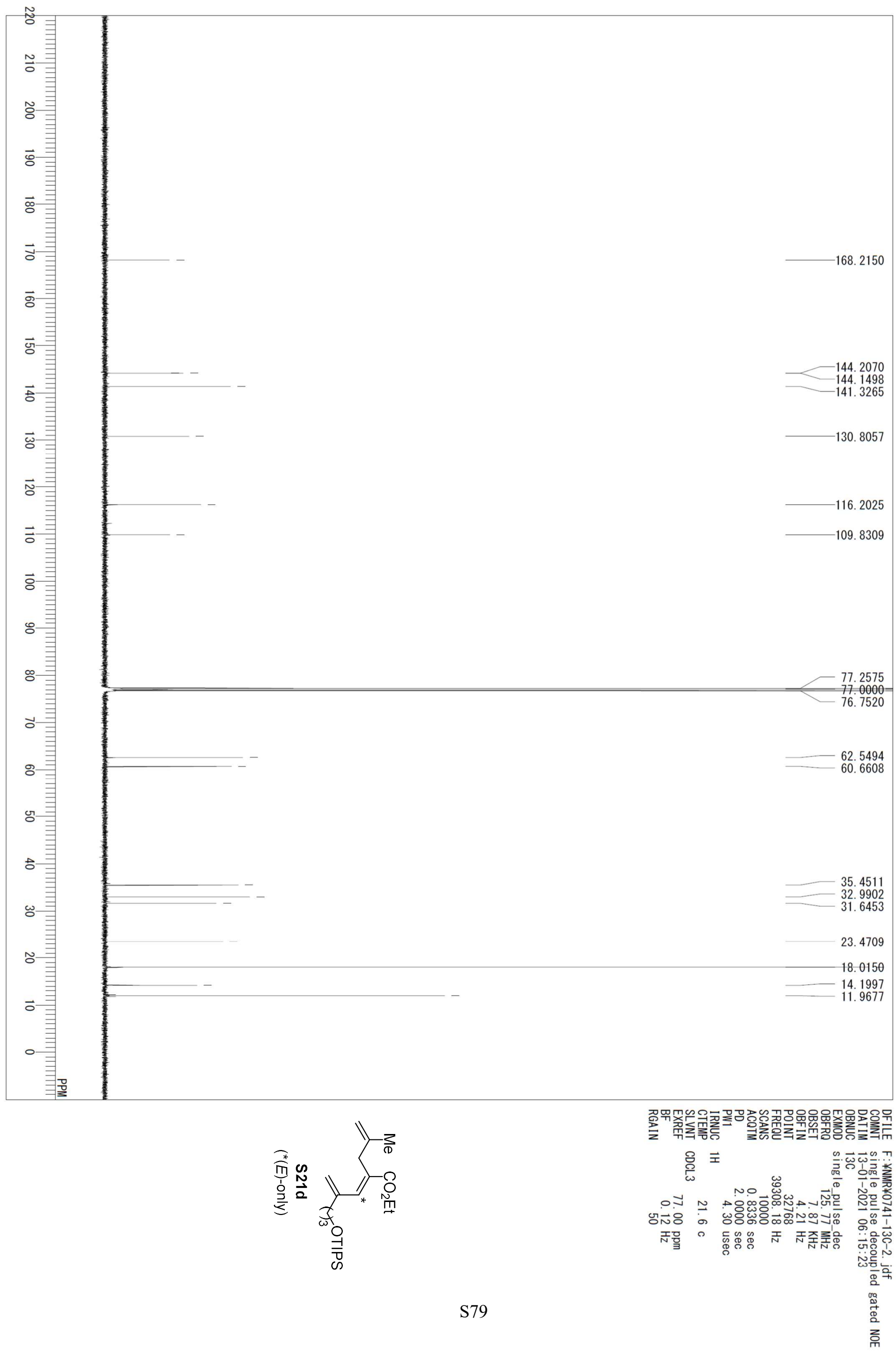




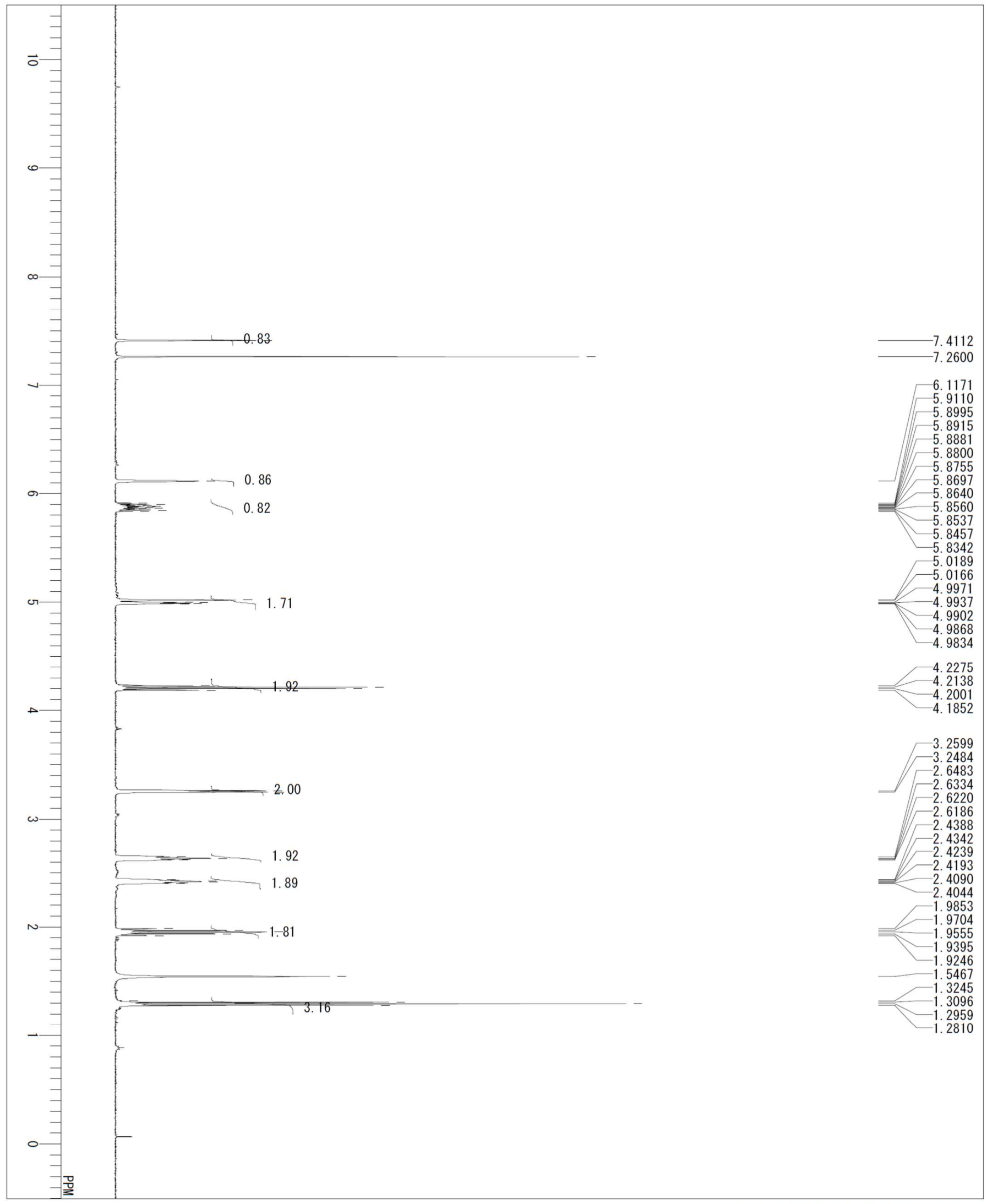

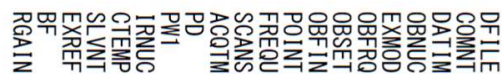

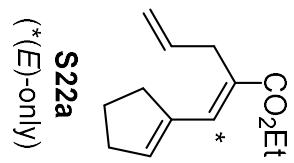

$\stackrel{8}{\mathrm{C}}$

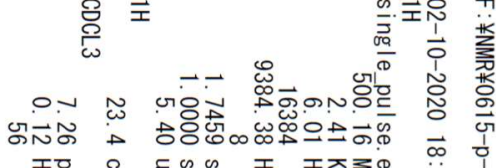

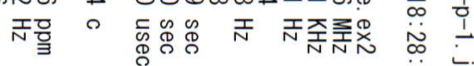




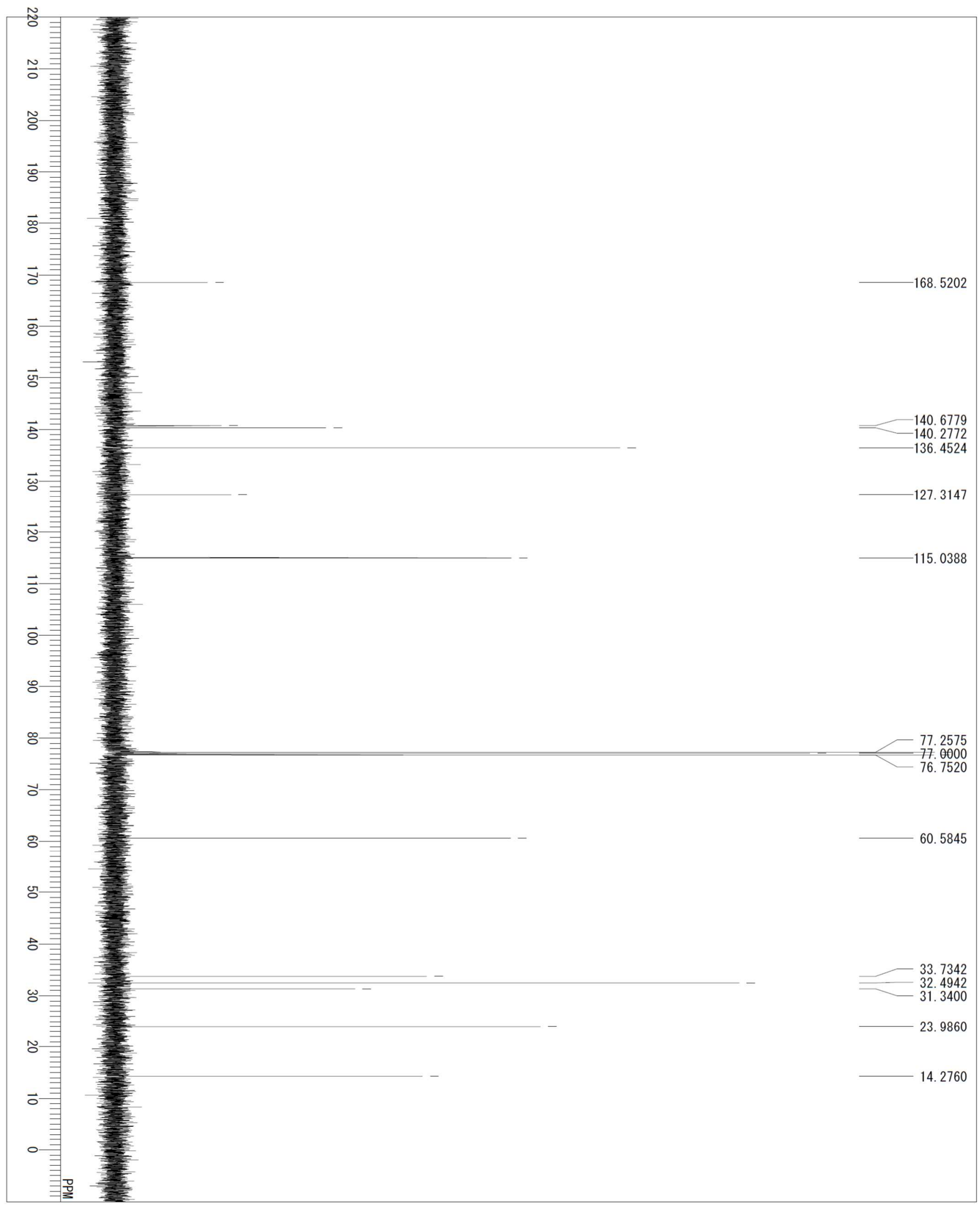

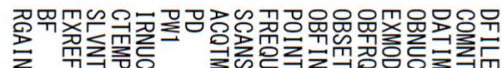

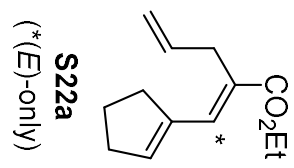

总 $\overline{1}$

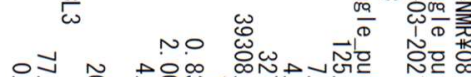
जति

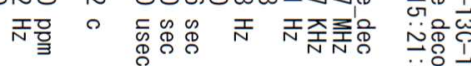



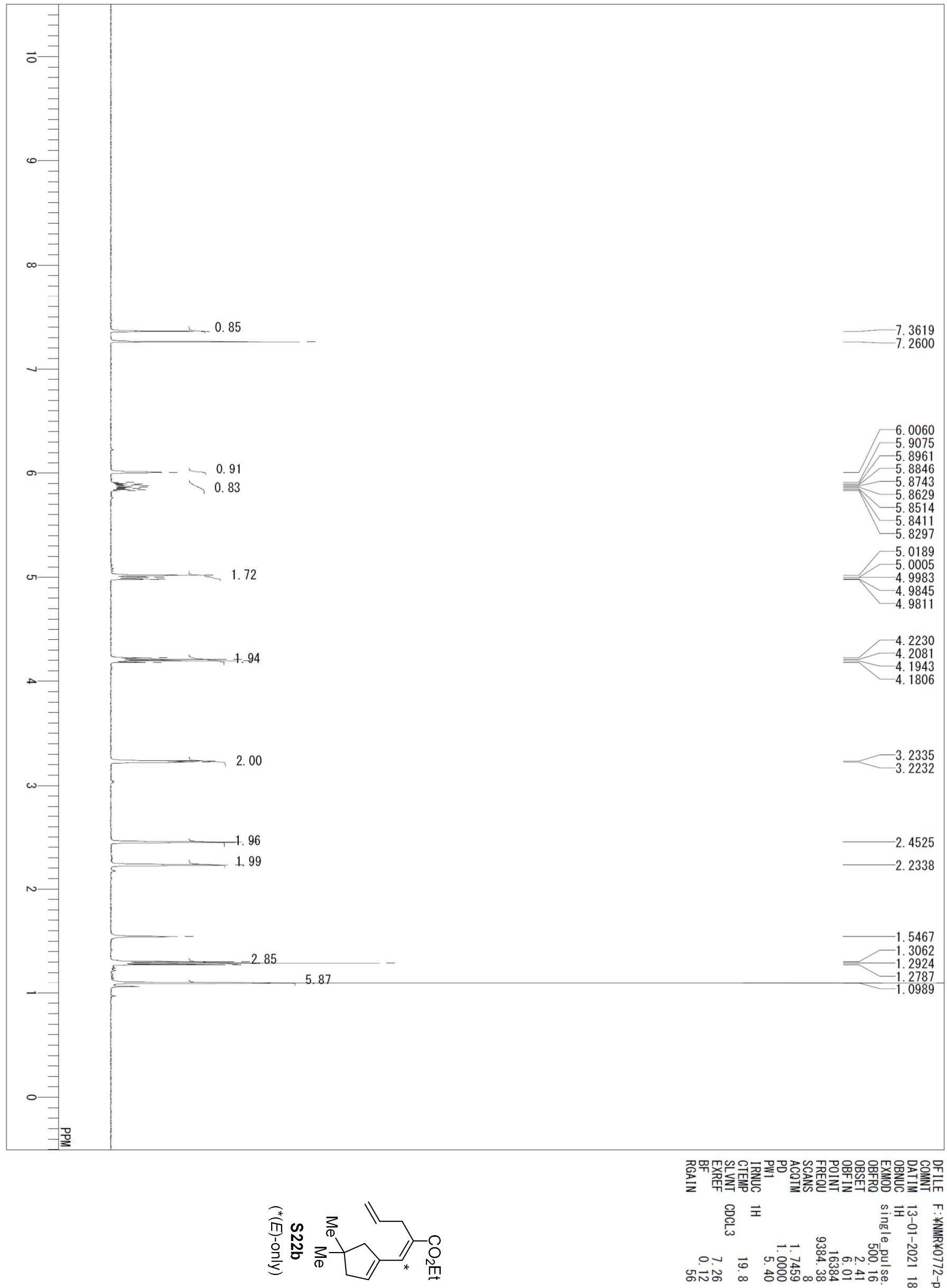

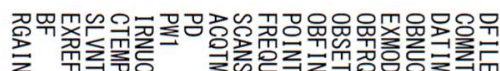

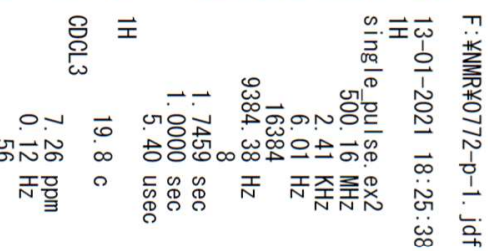



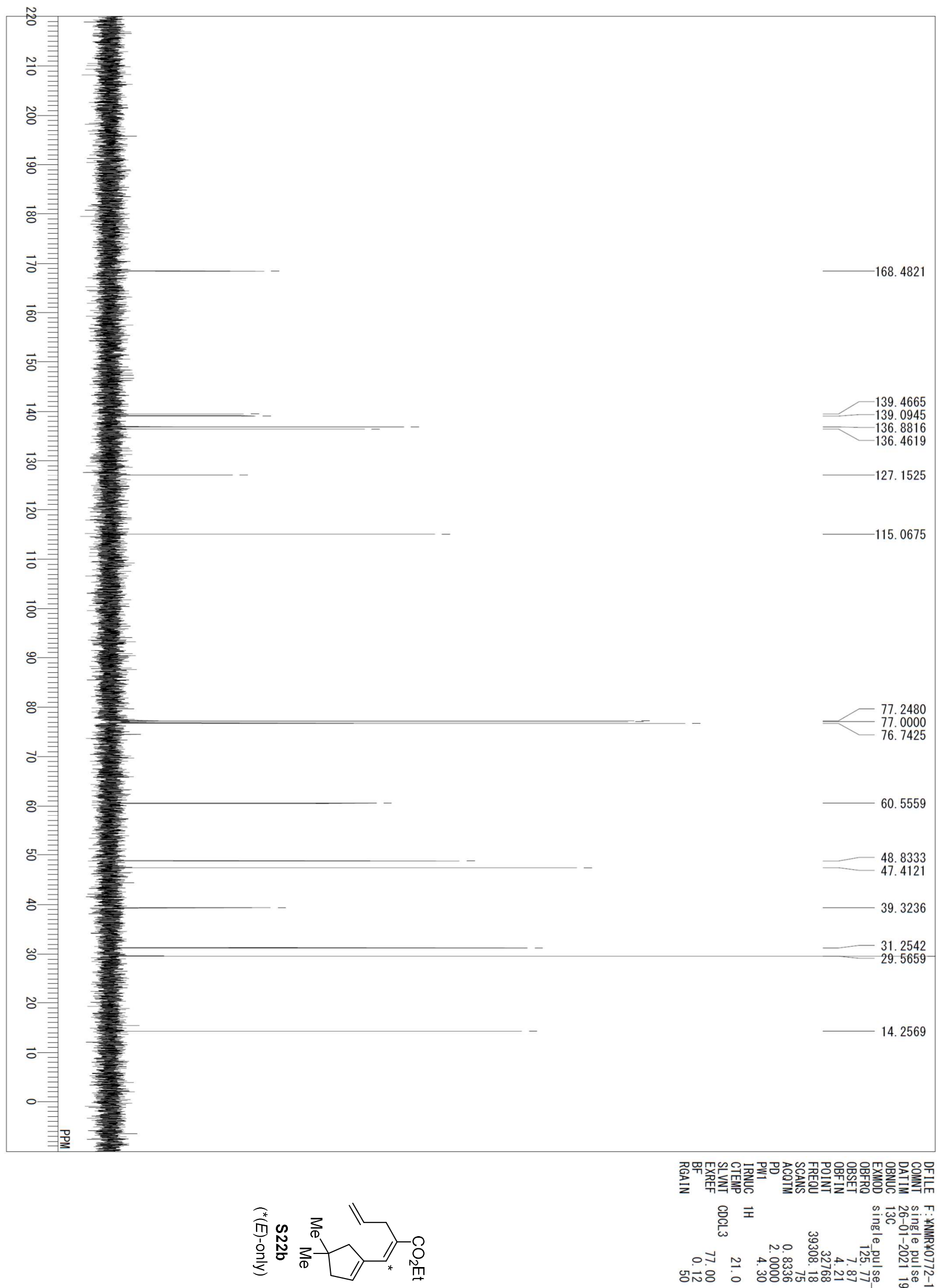

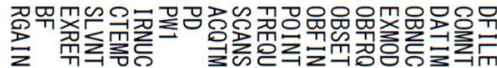

욤포 no

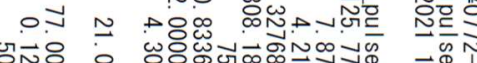

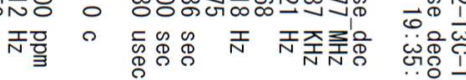




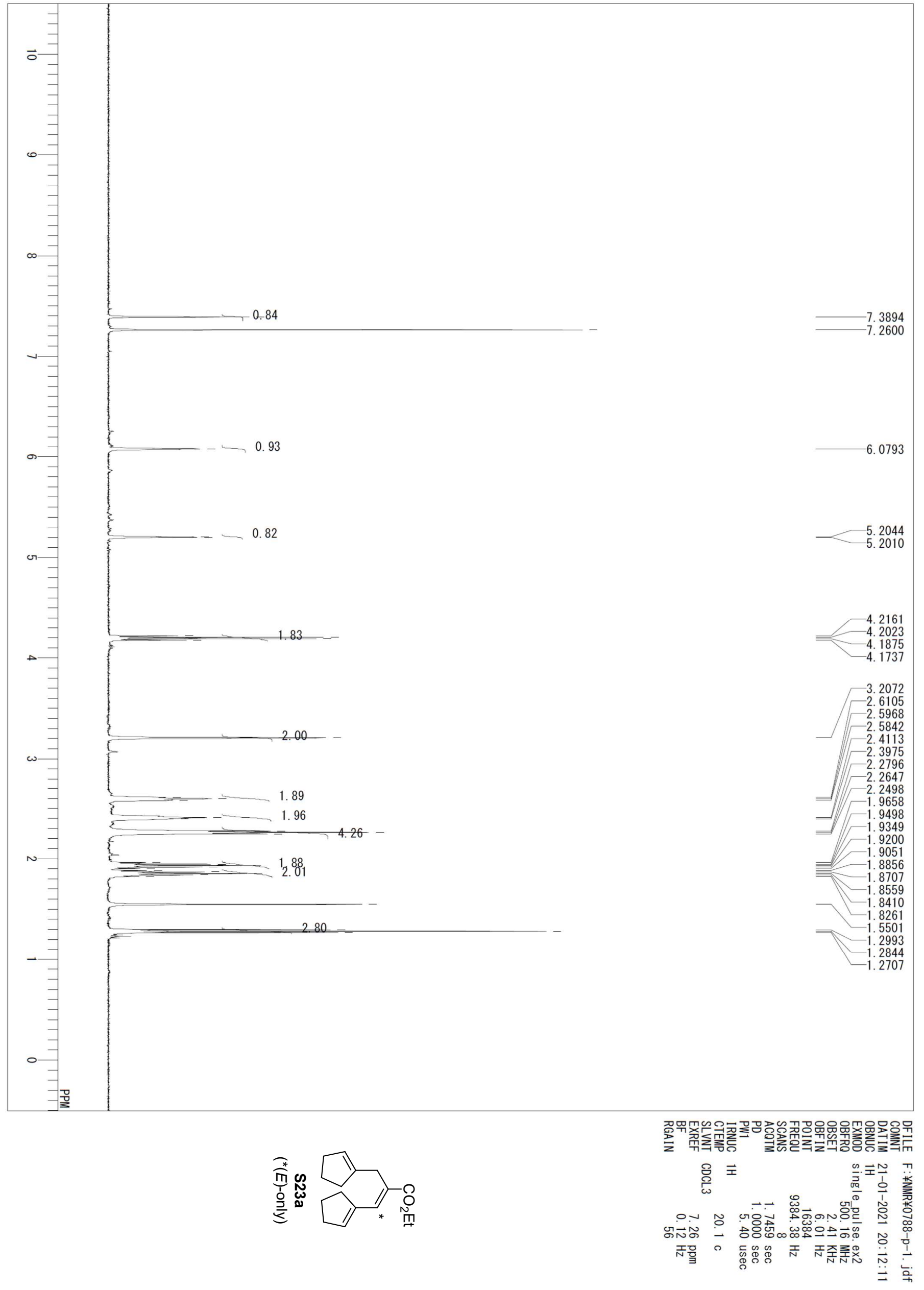



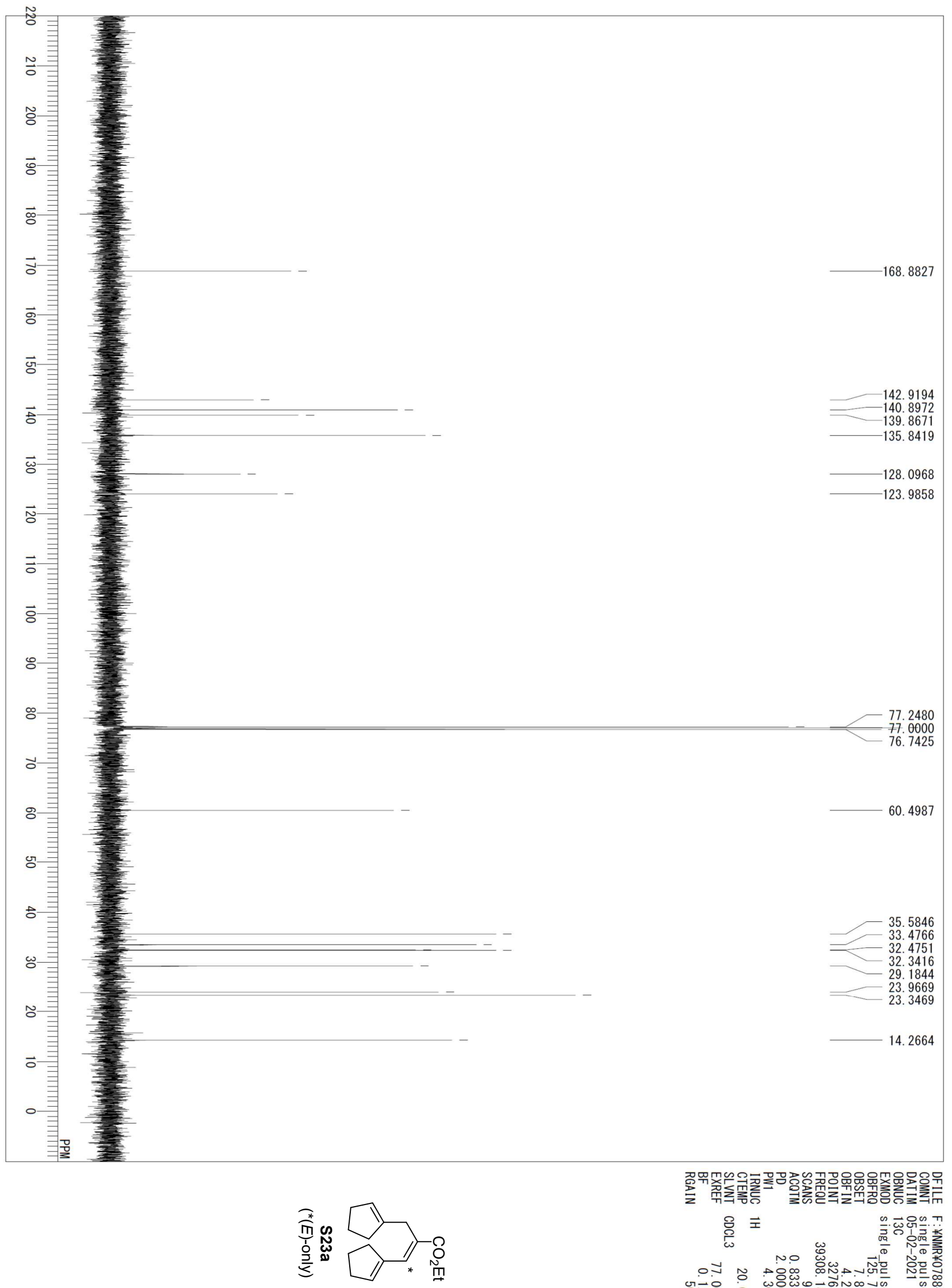

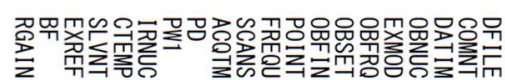

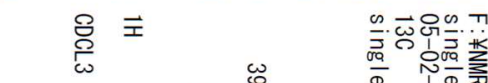

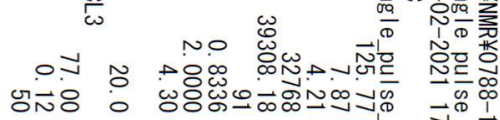

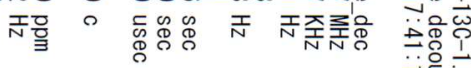



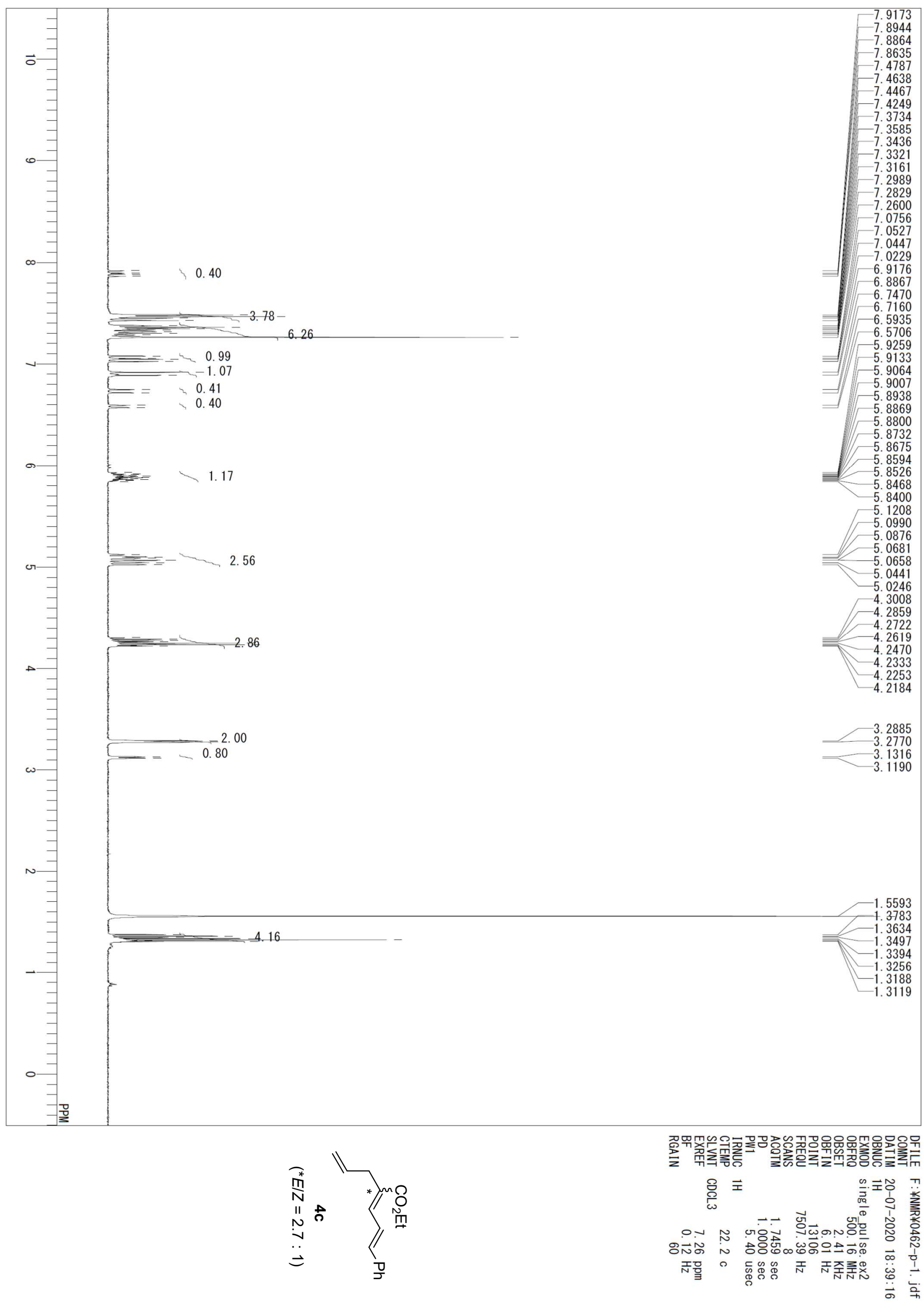


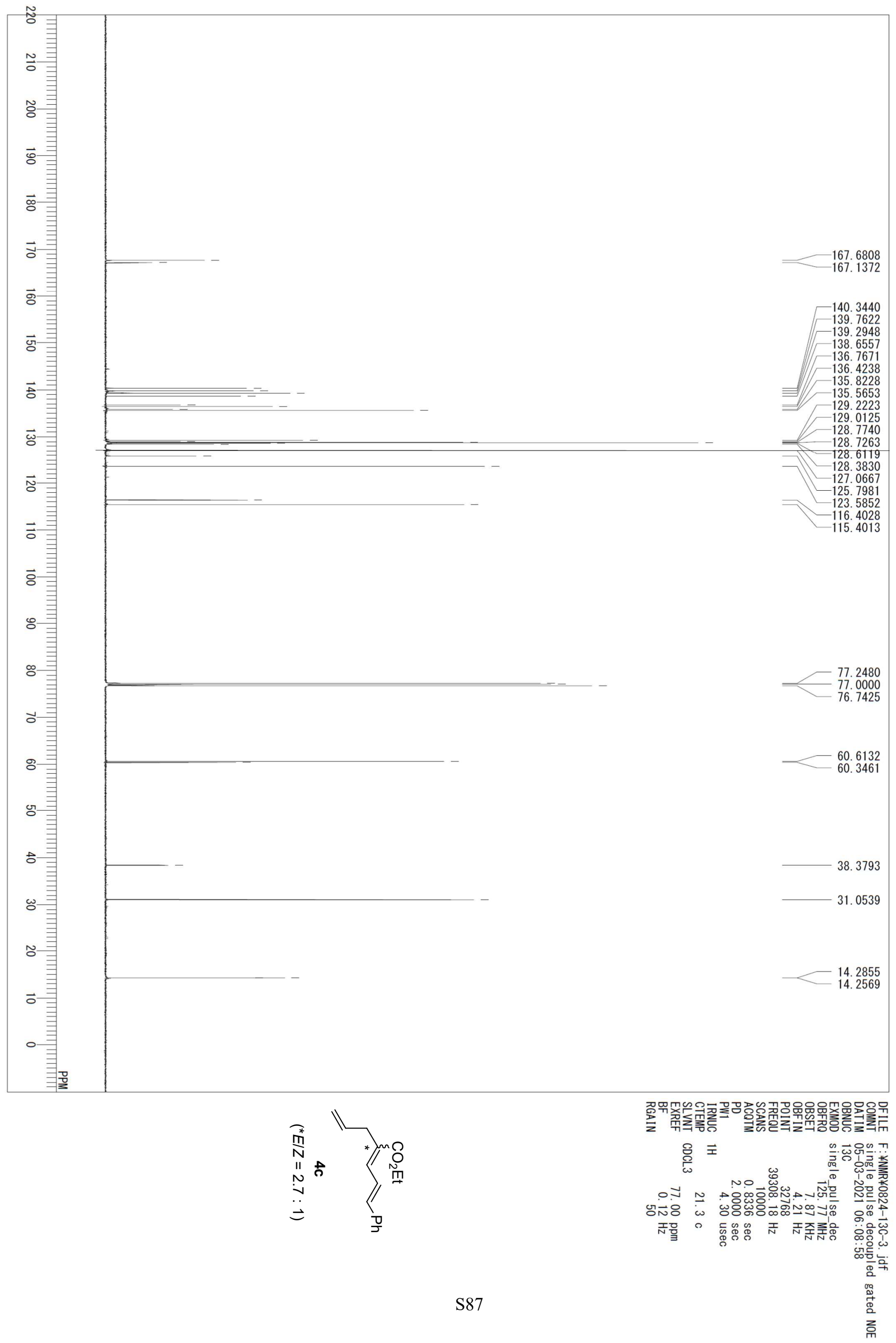



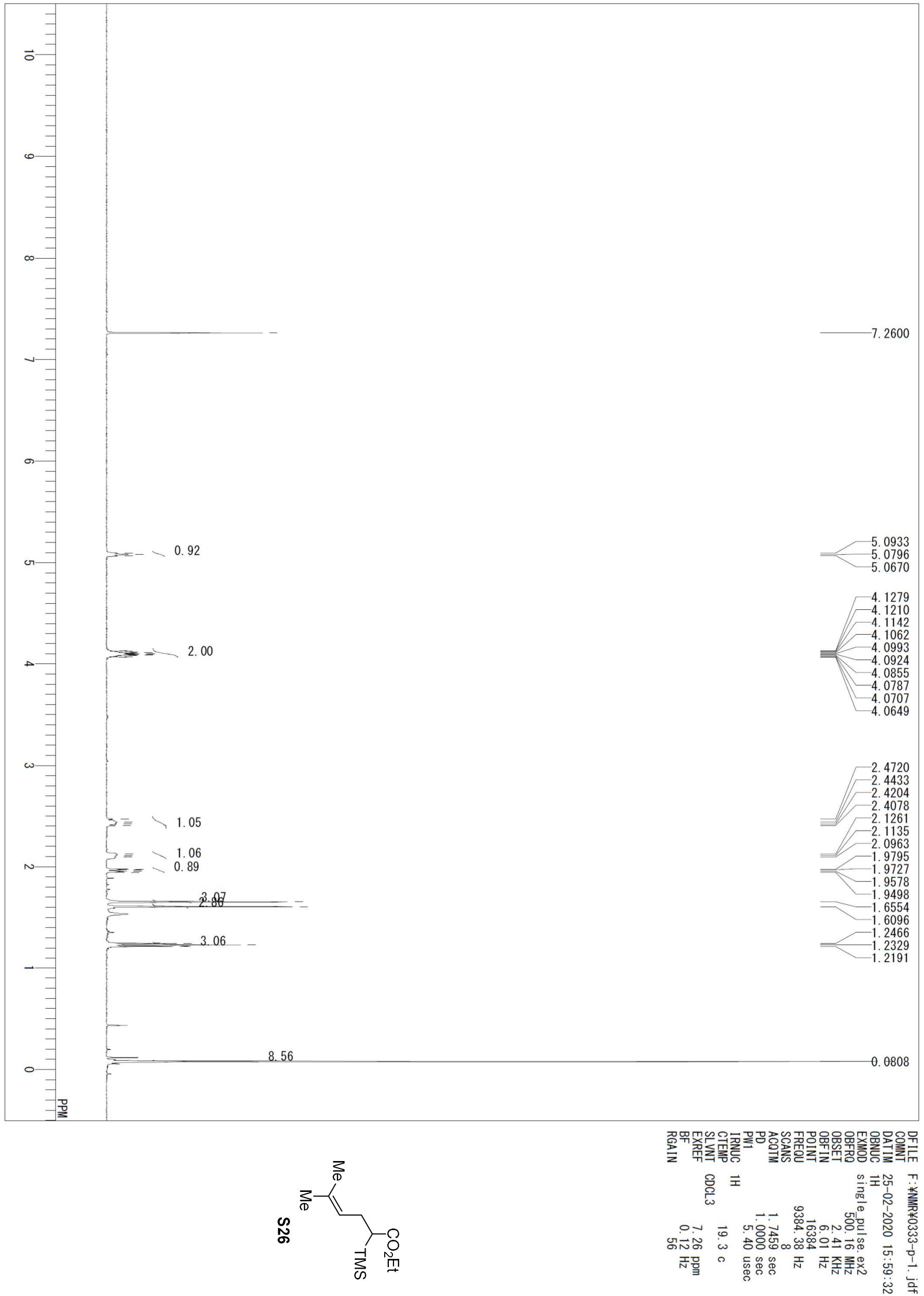


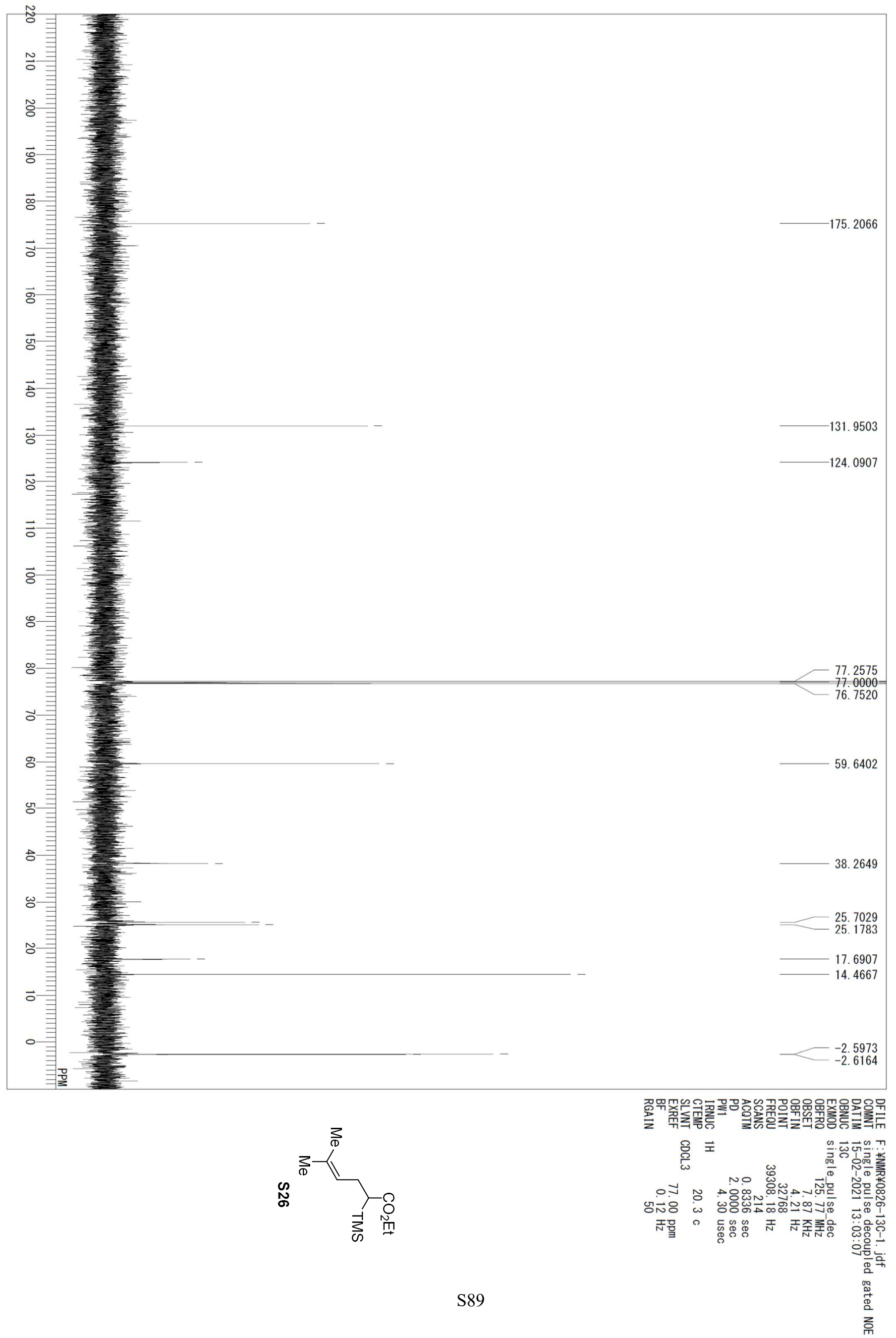



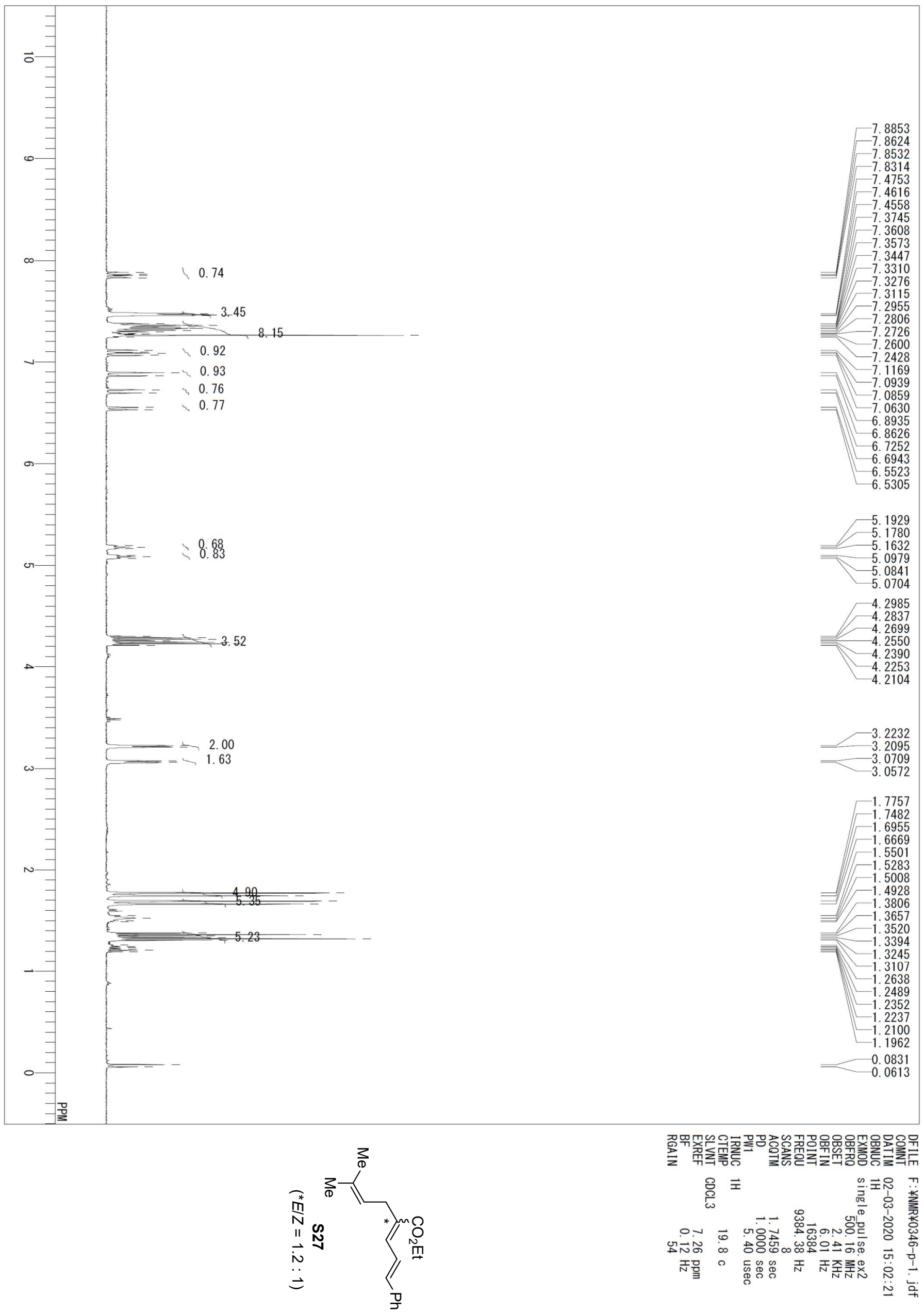

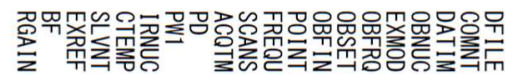

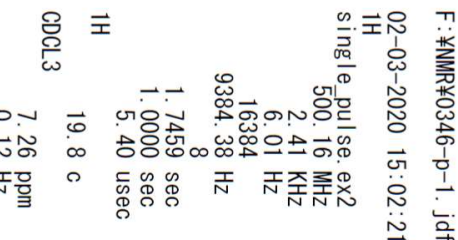




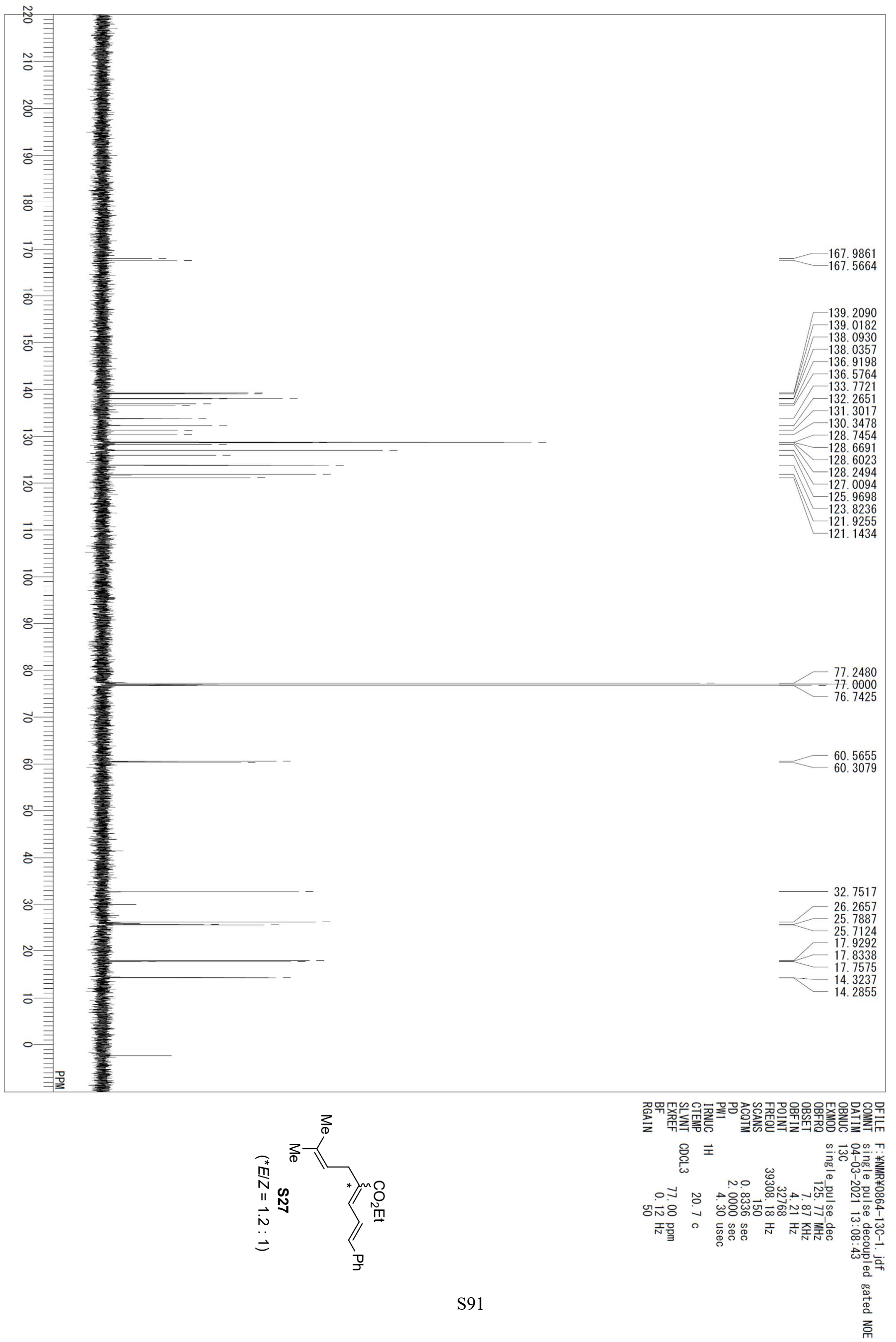




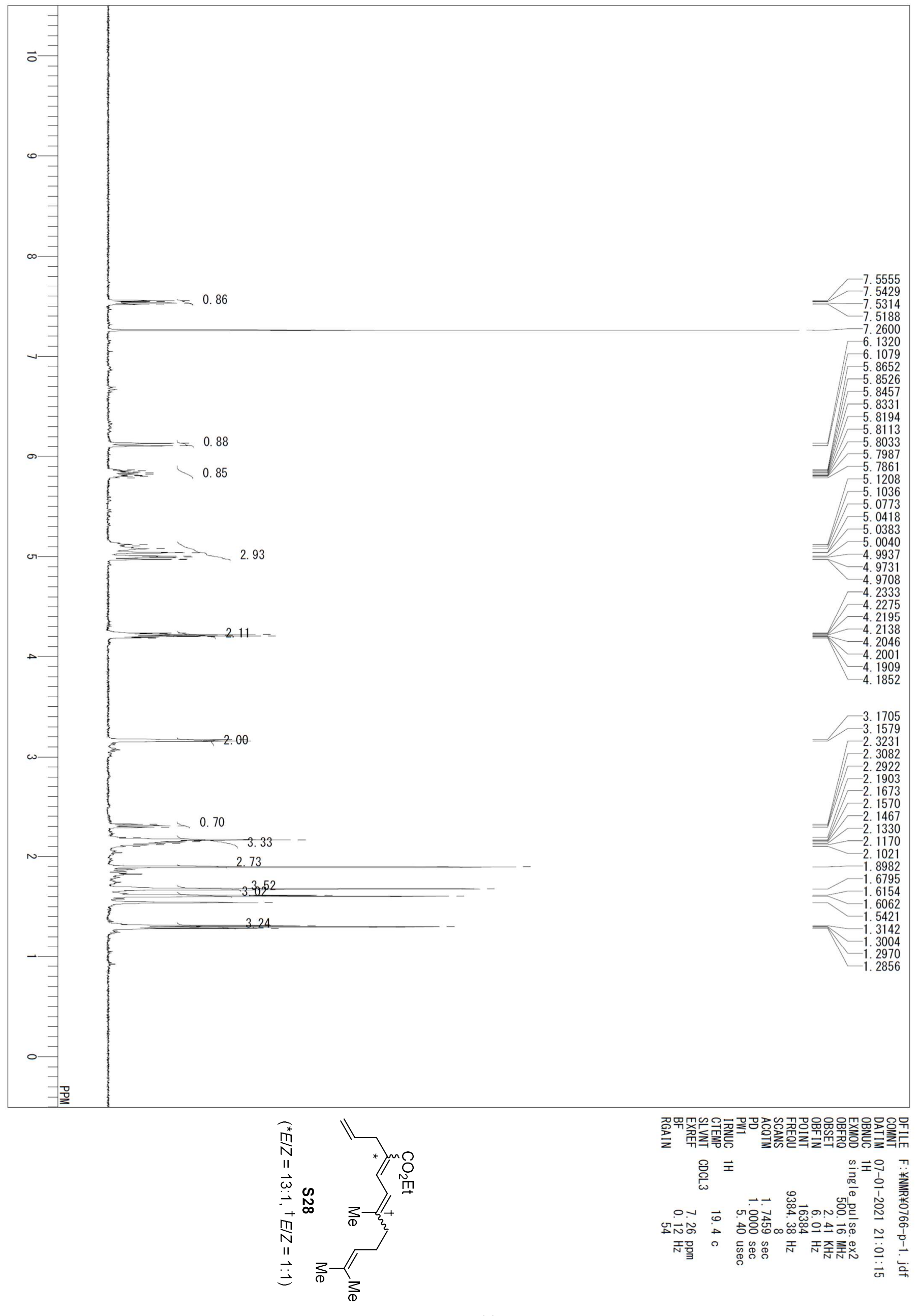




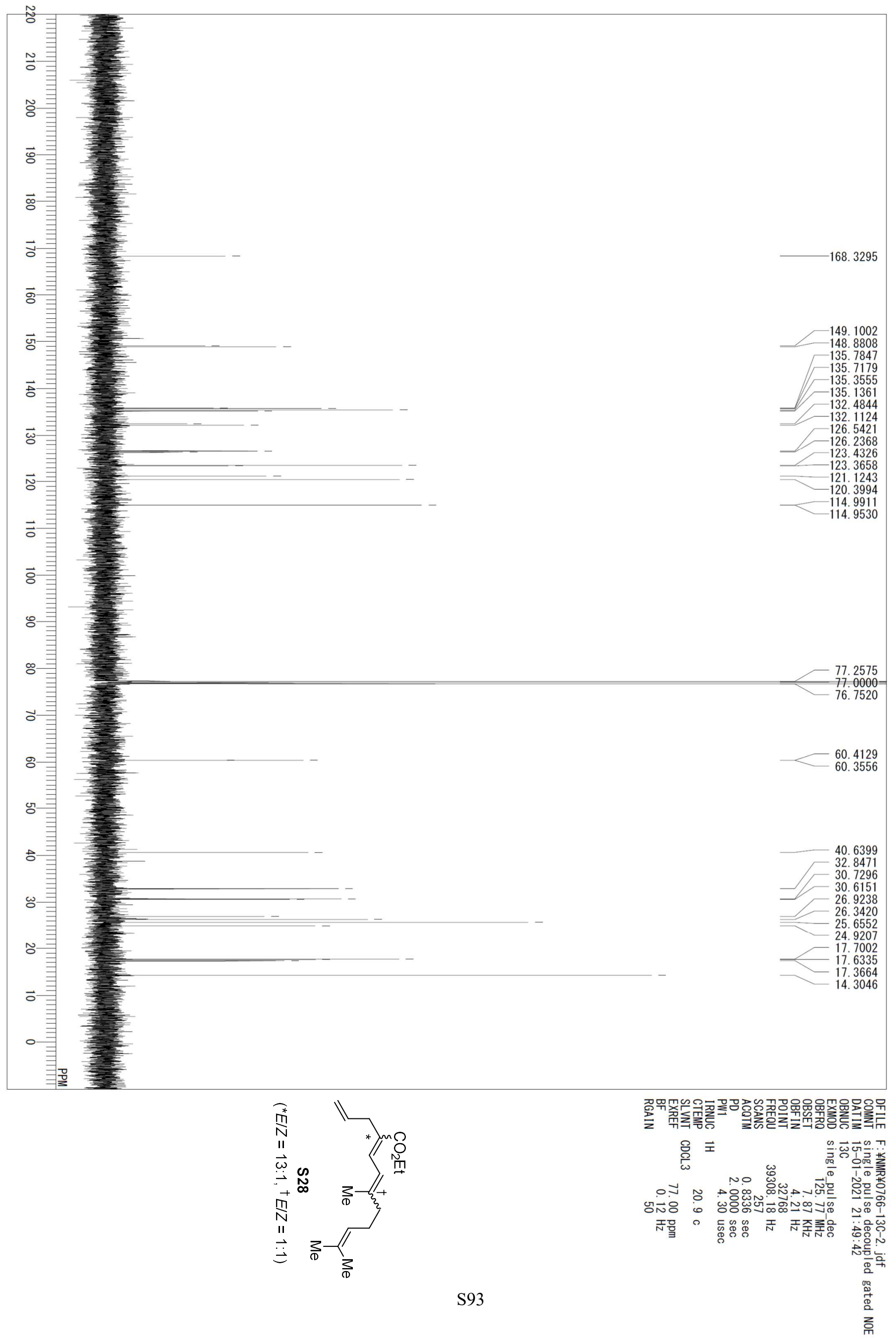



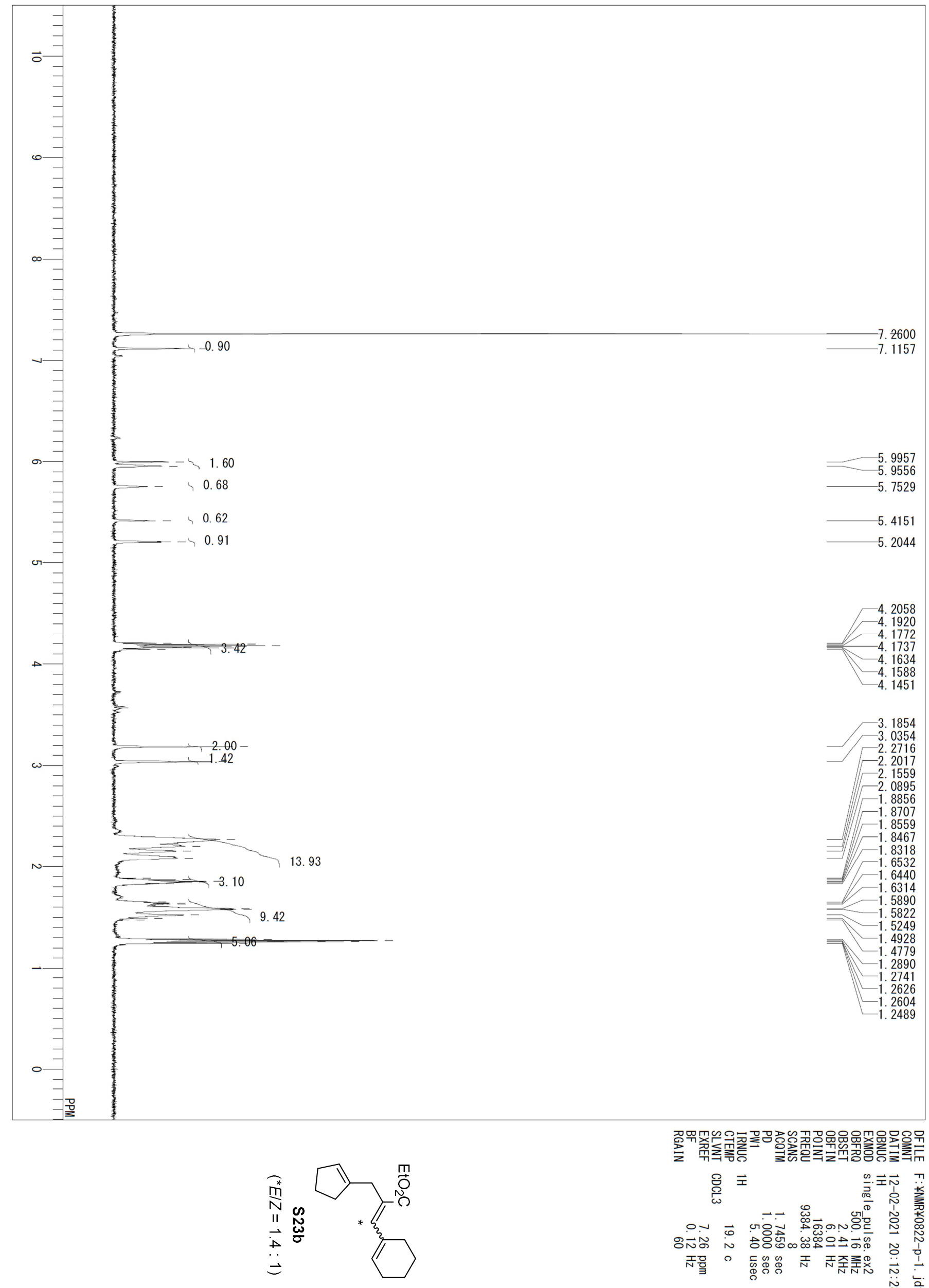

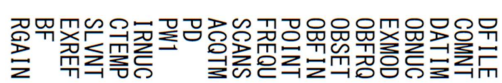

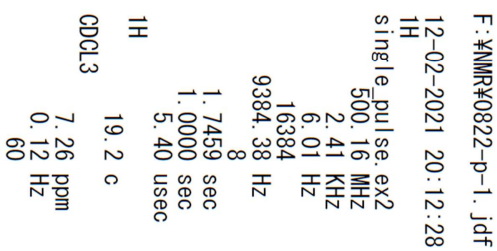




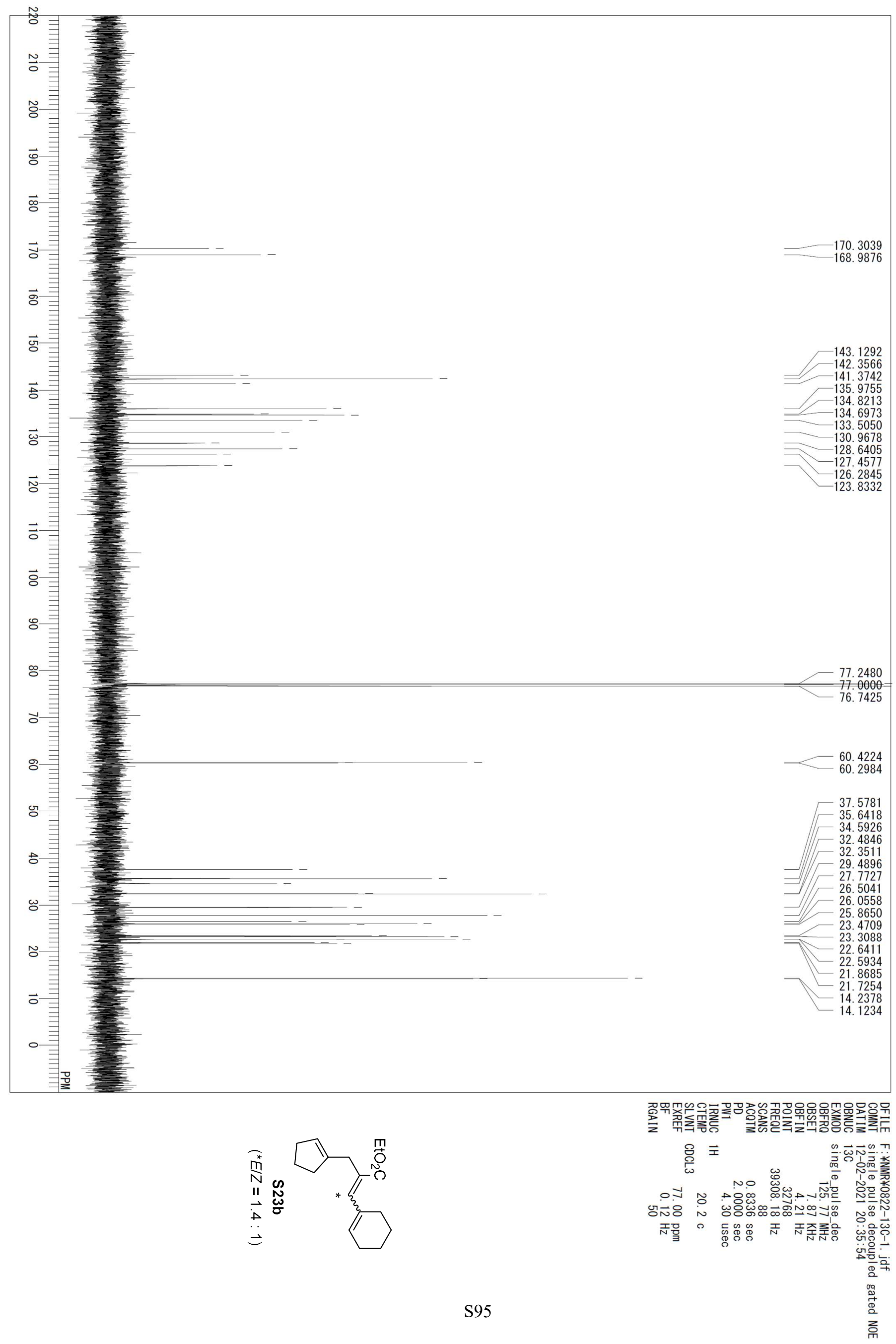



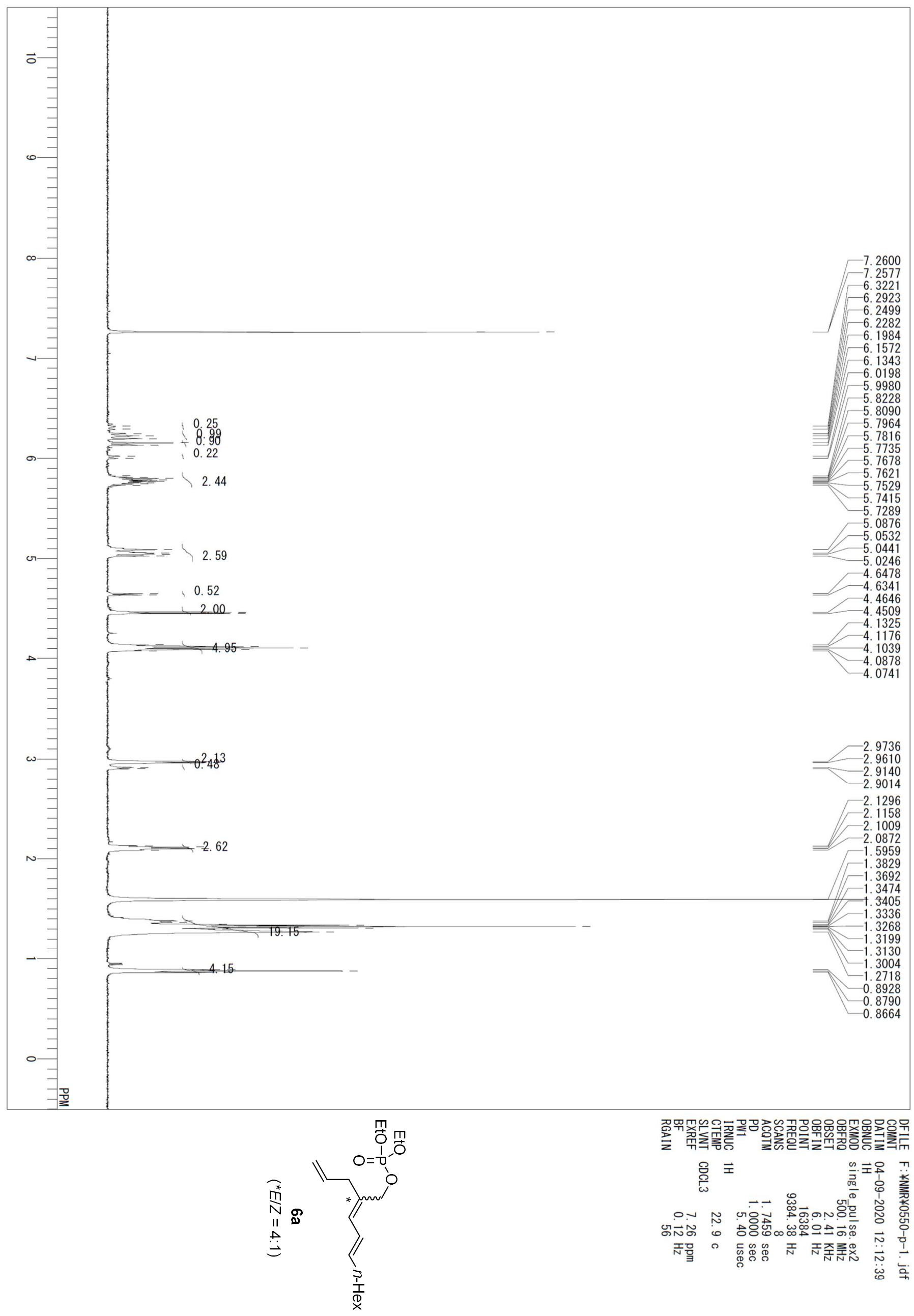

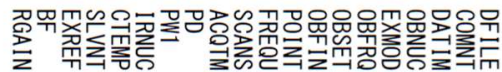

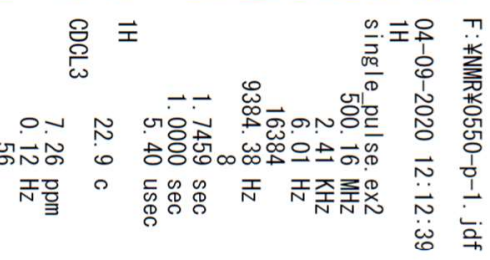




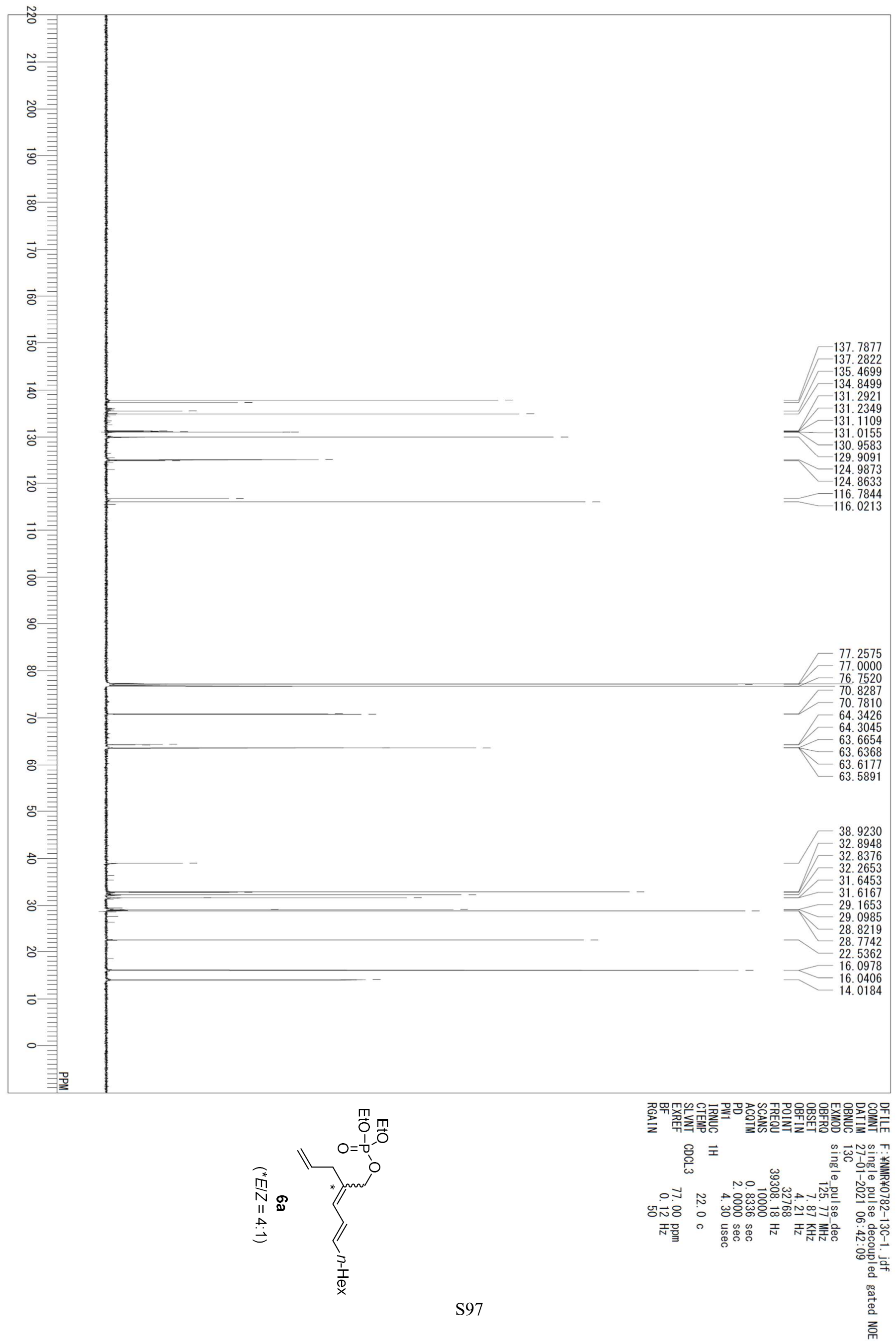




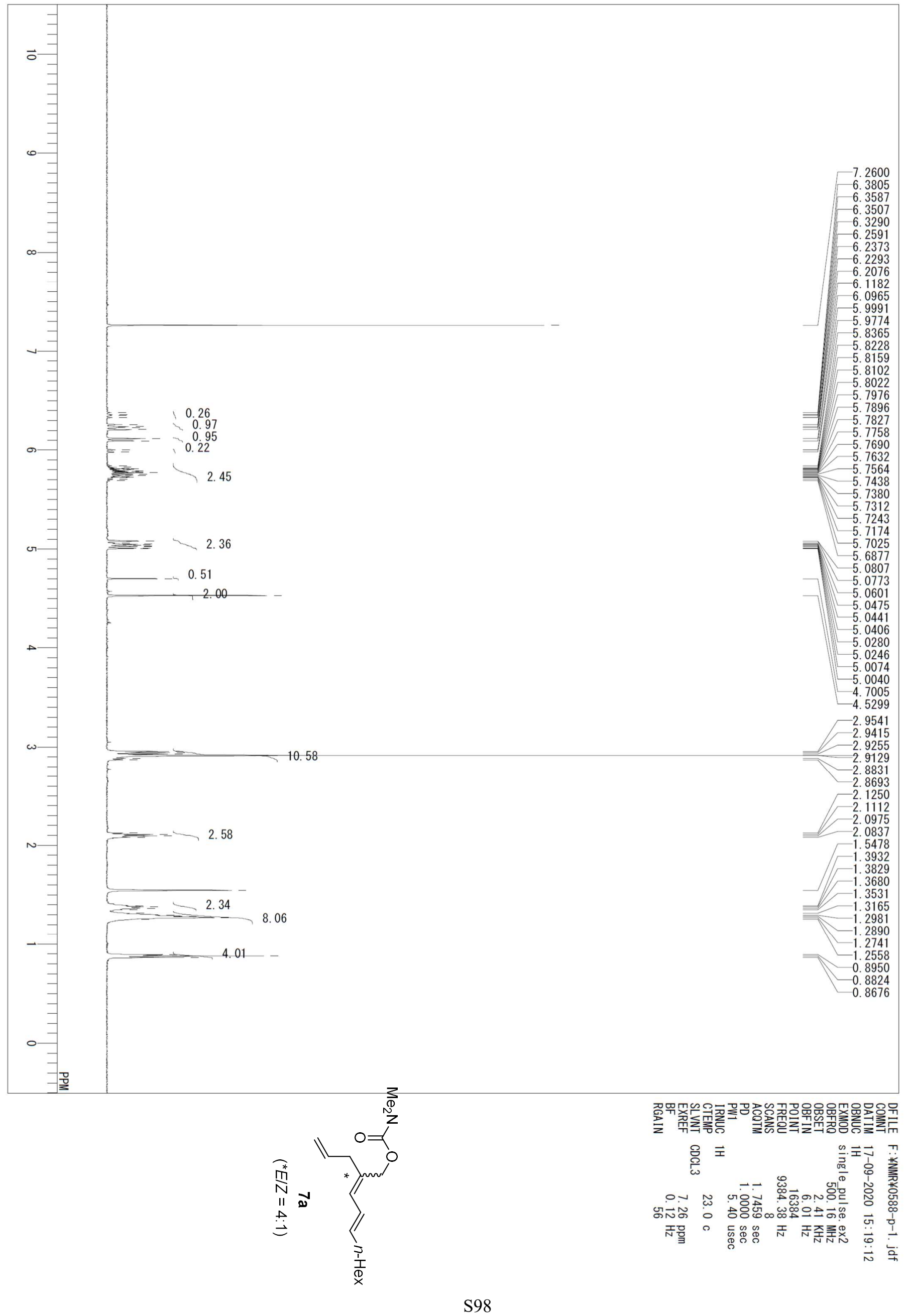




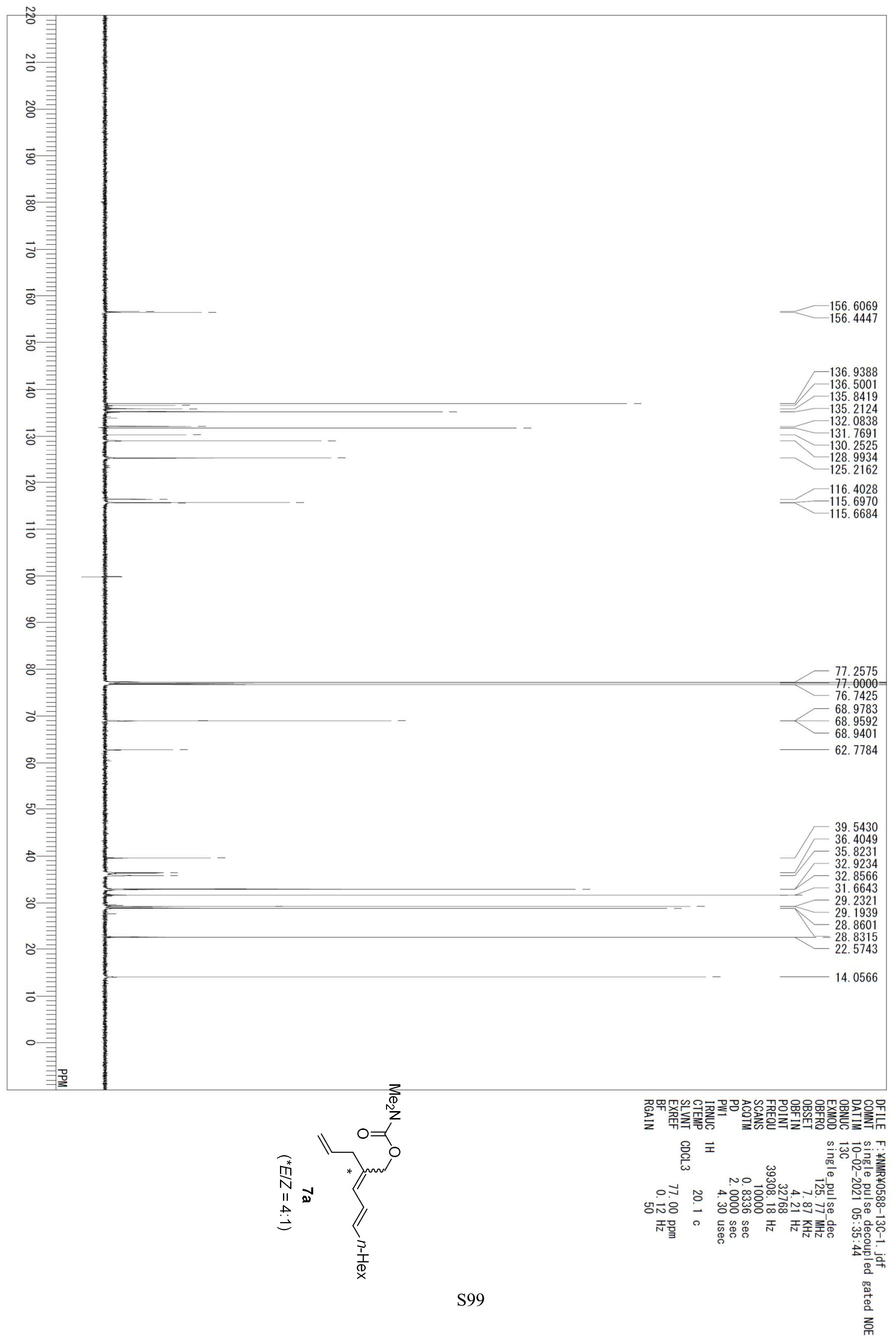




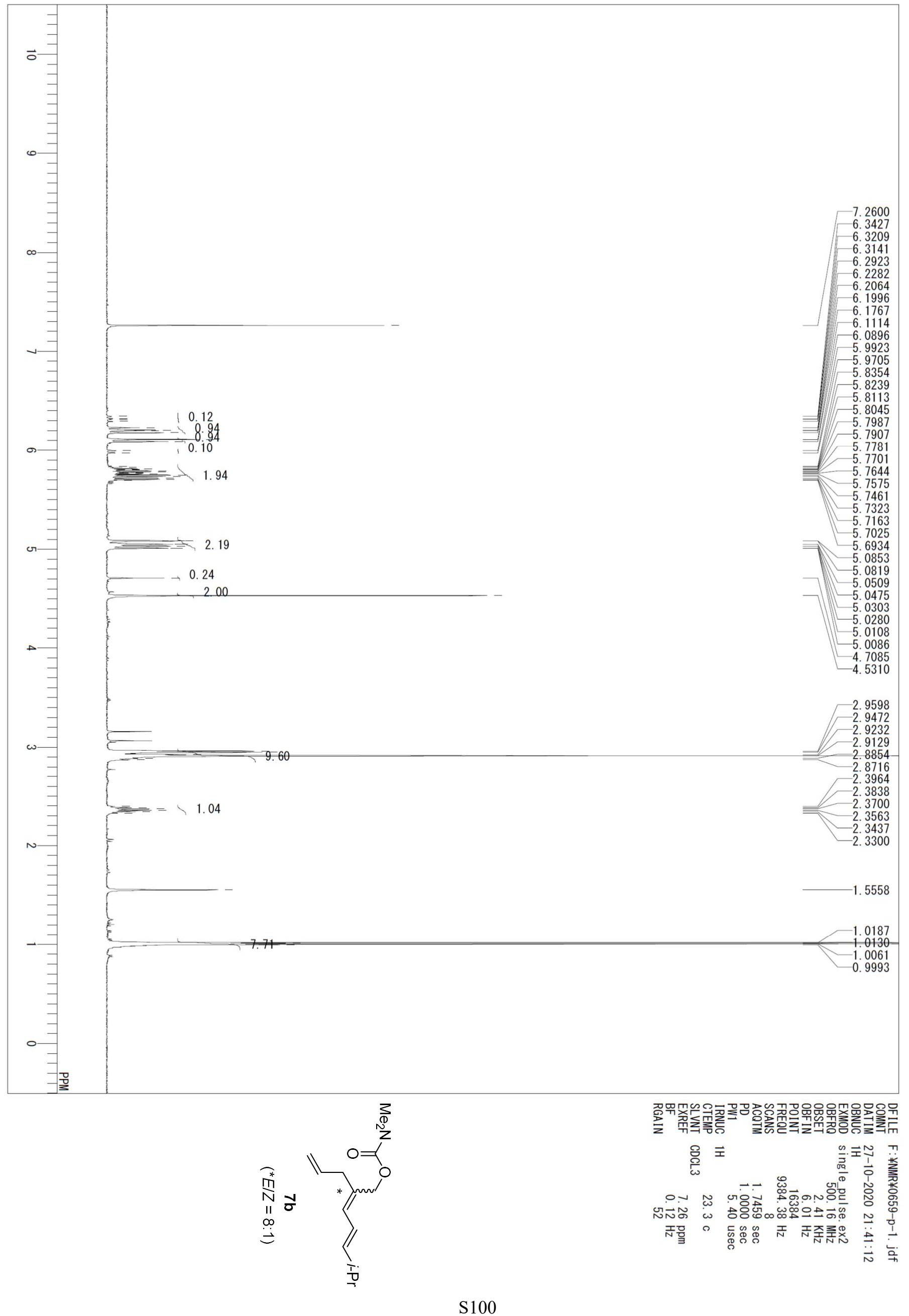




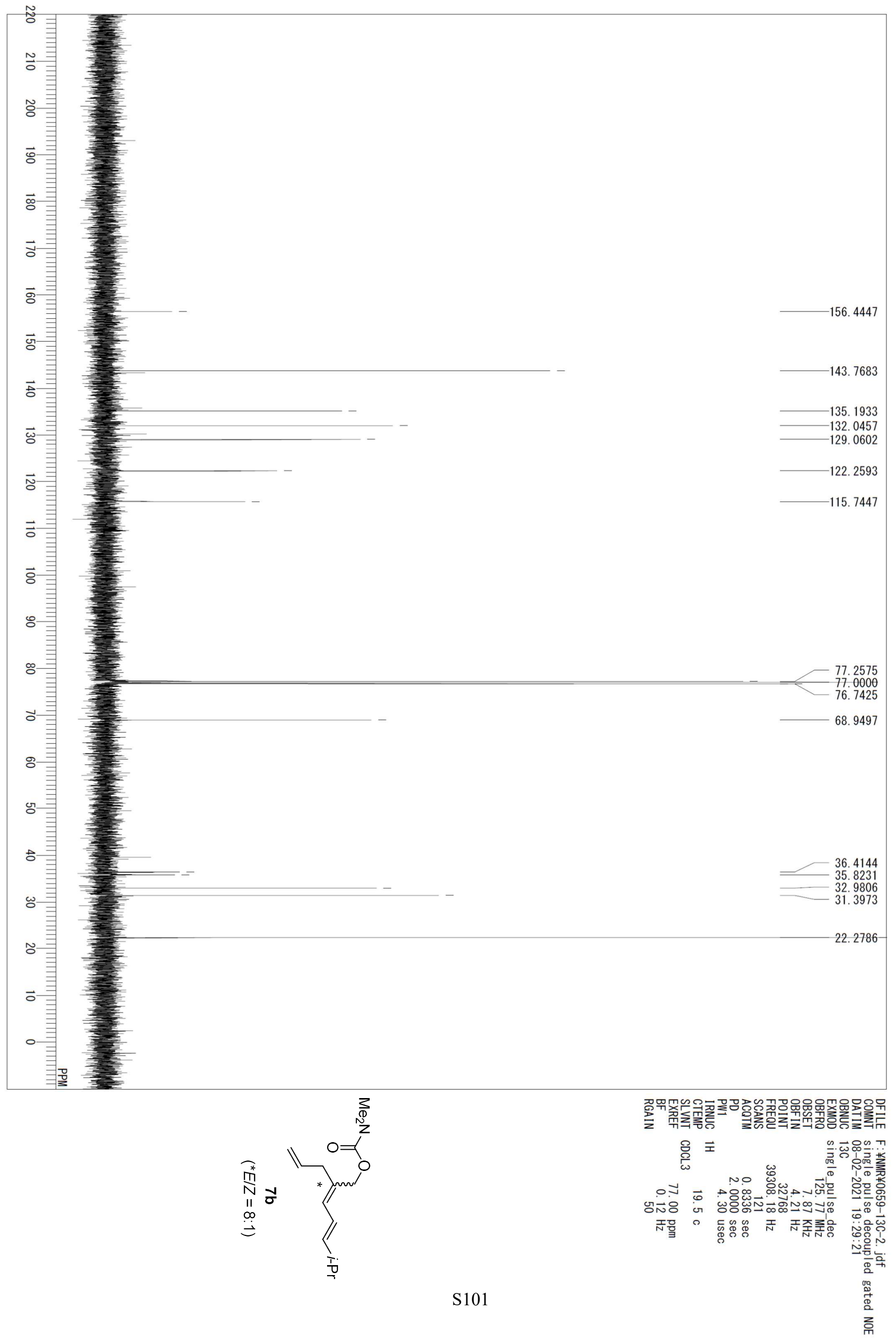




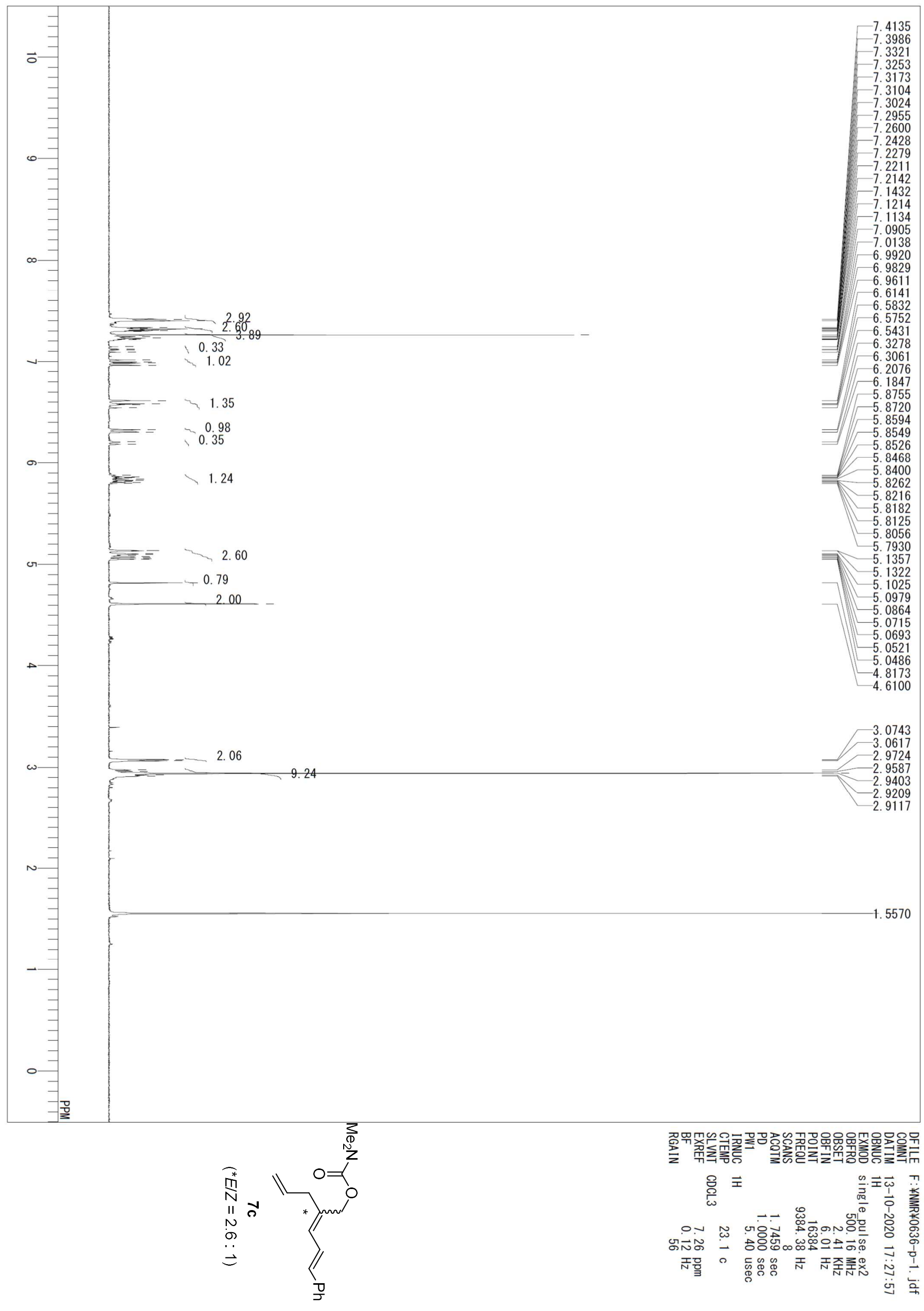




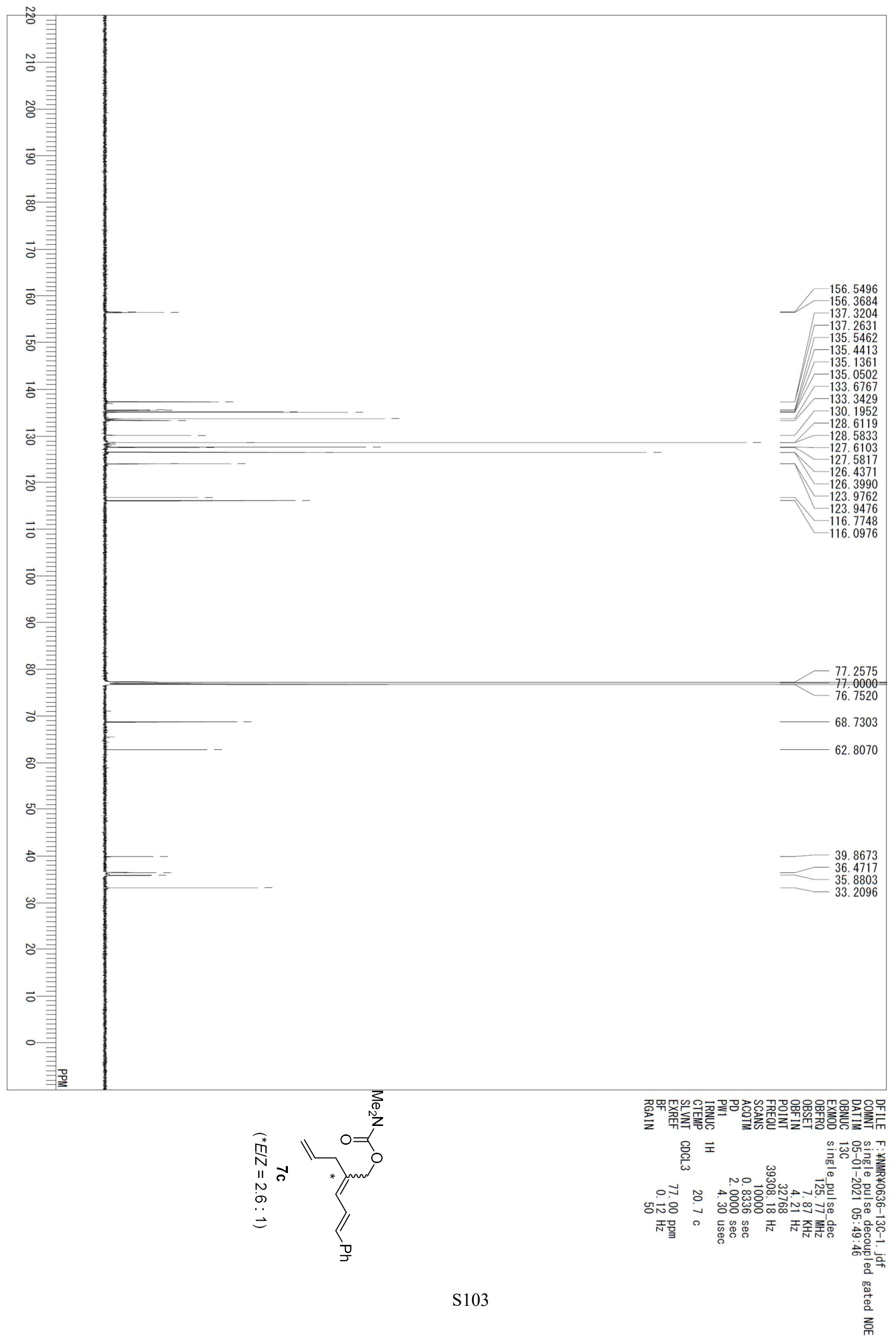



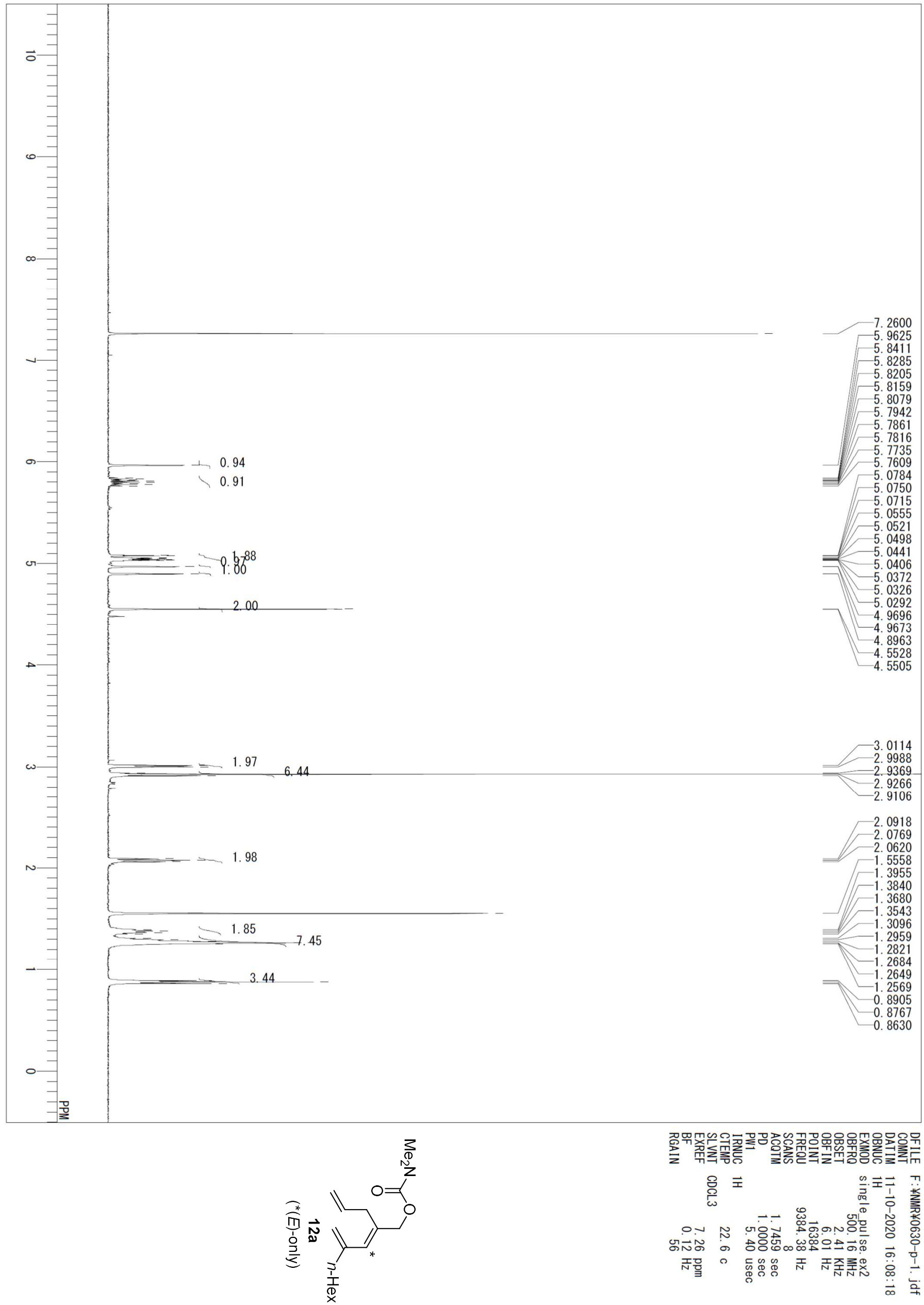

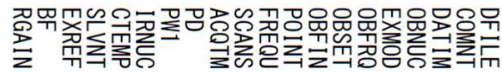

总

에 N

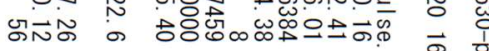

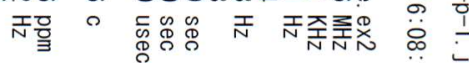



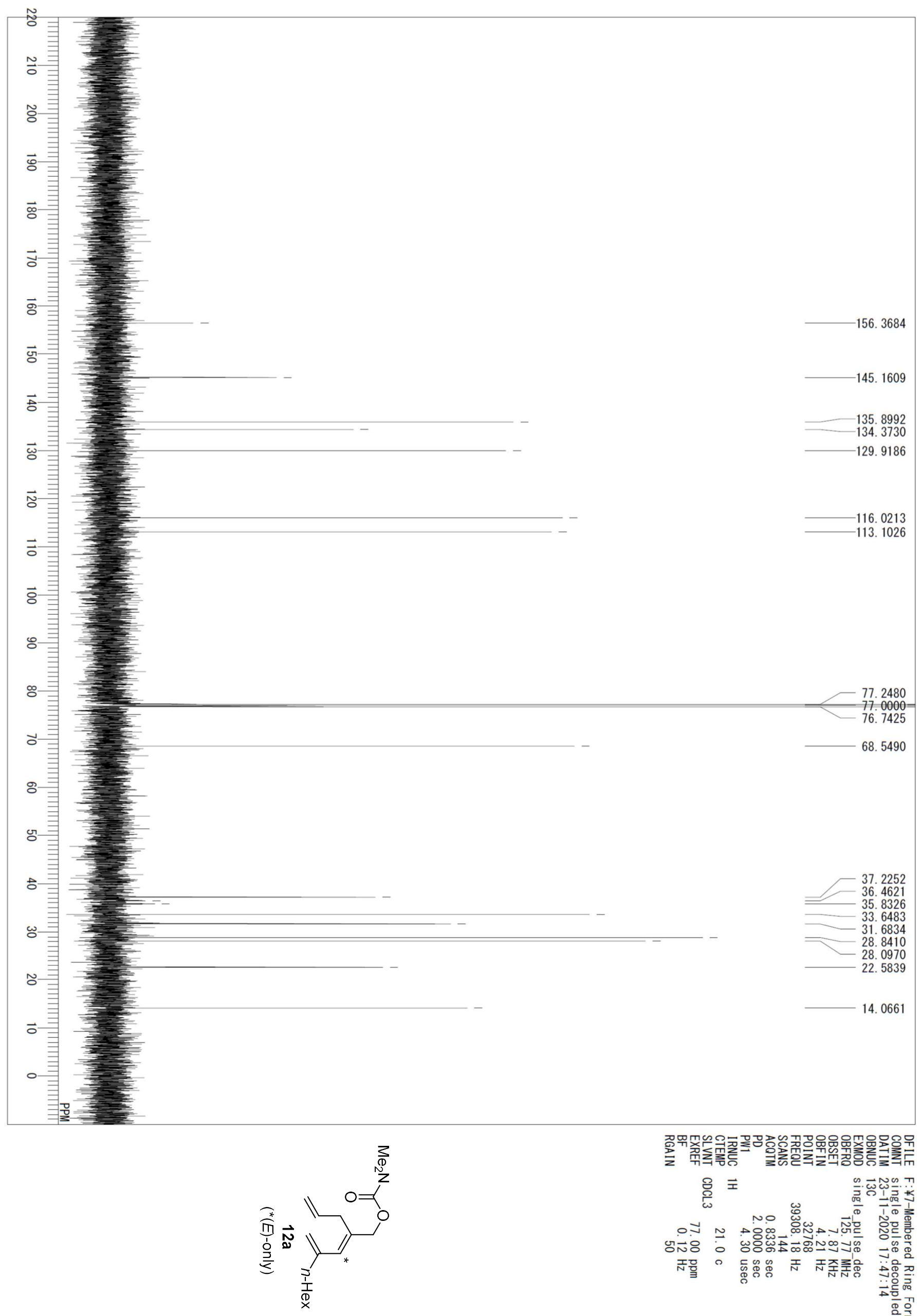

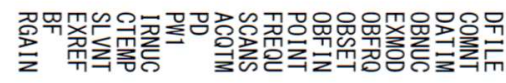

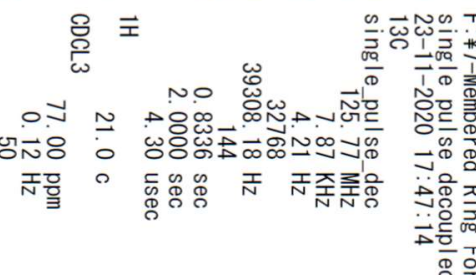




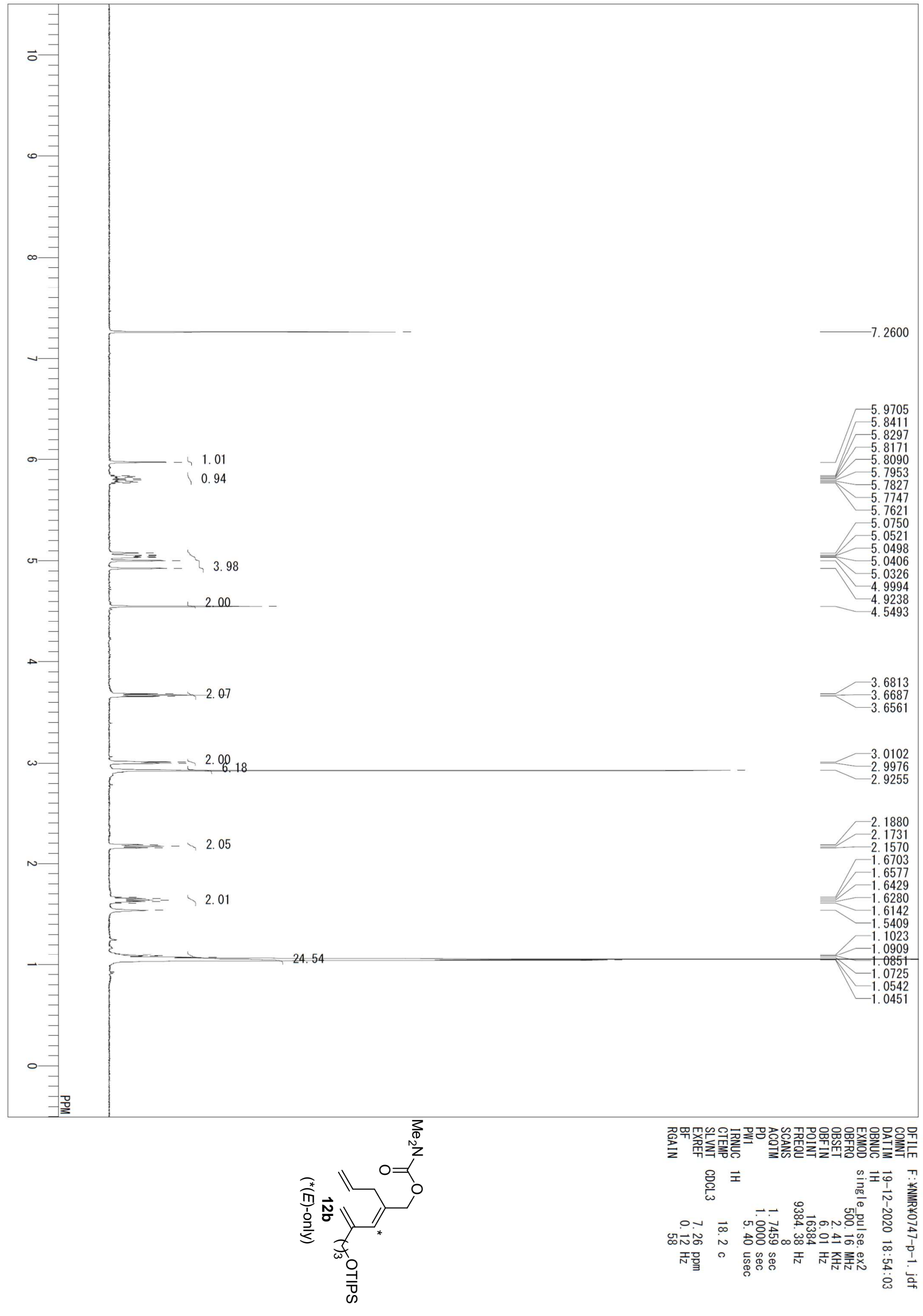




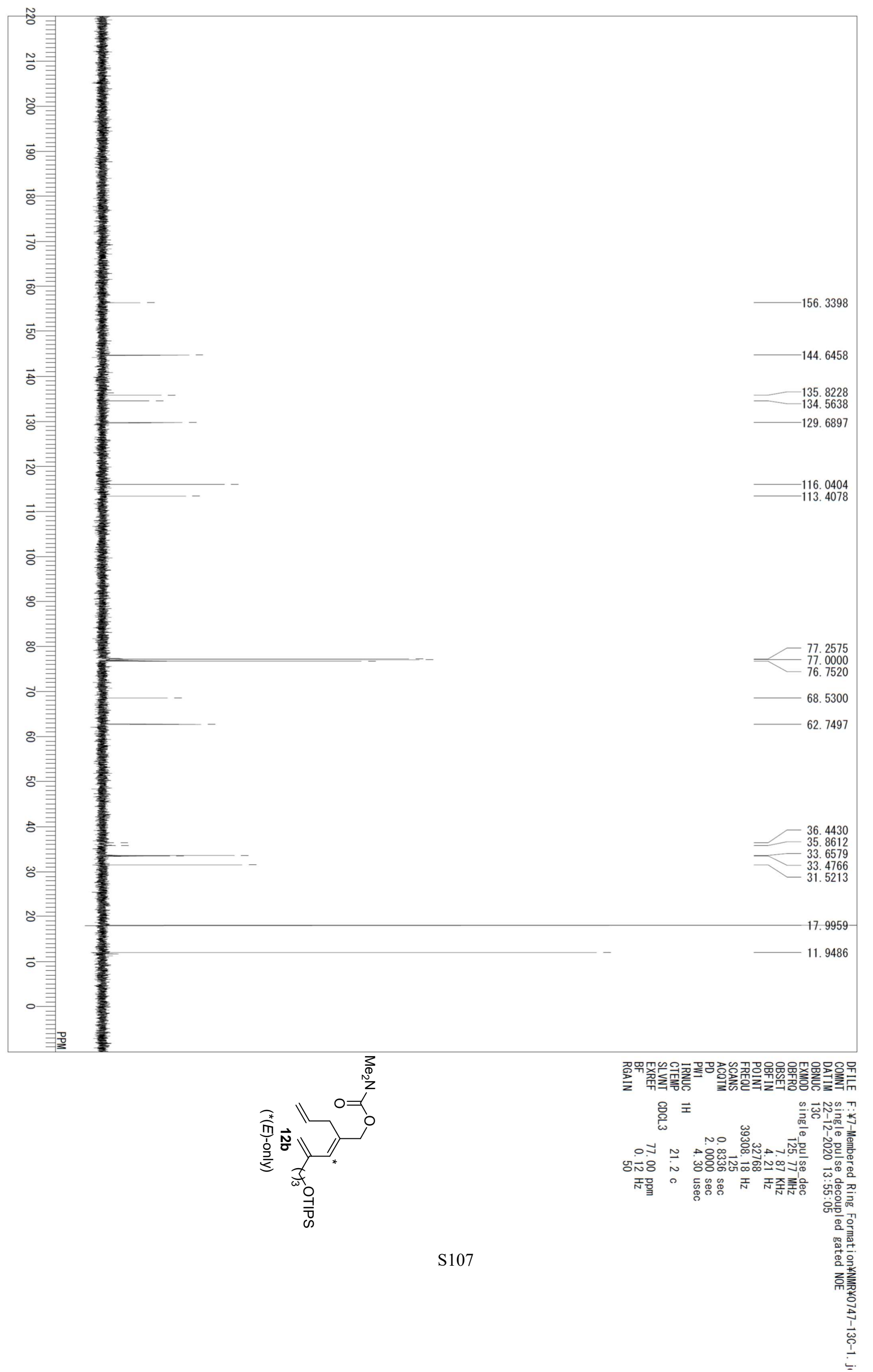



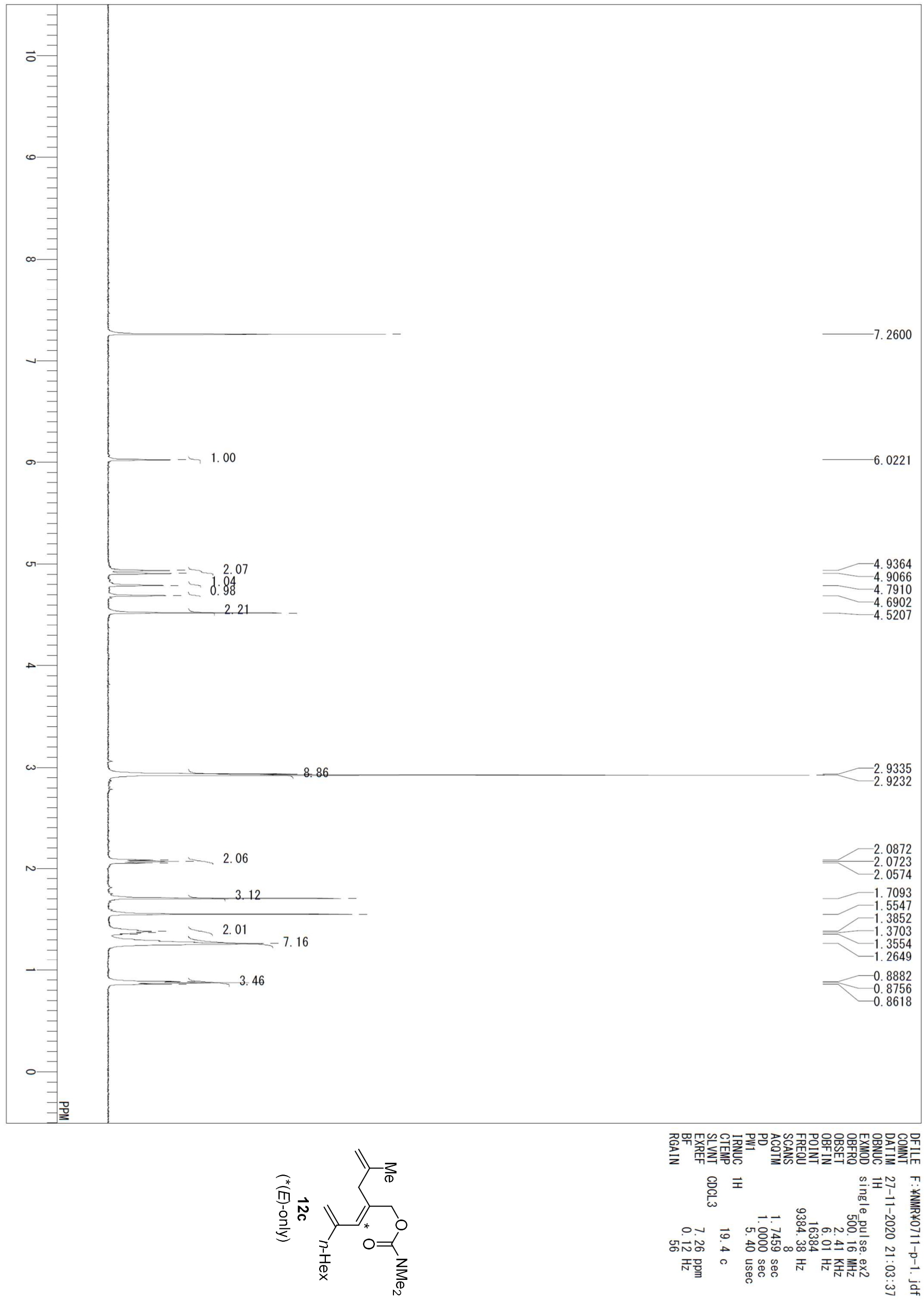

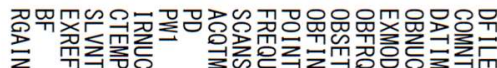

总

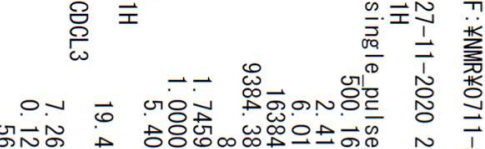

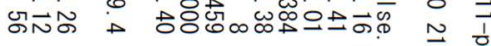

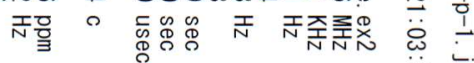



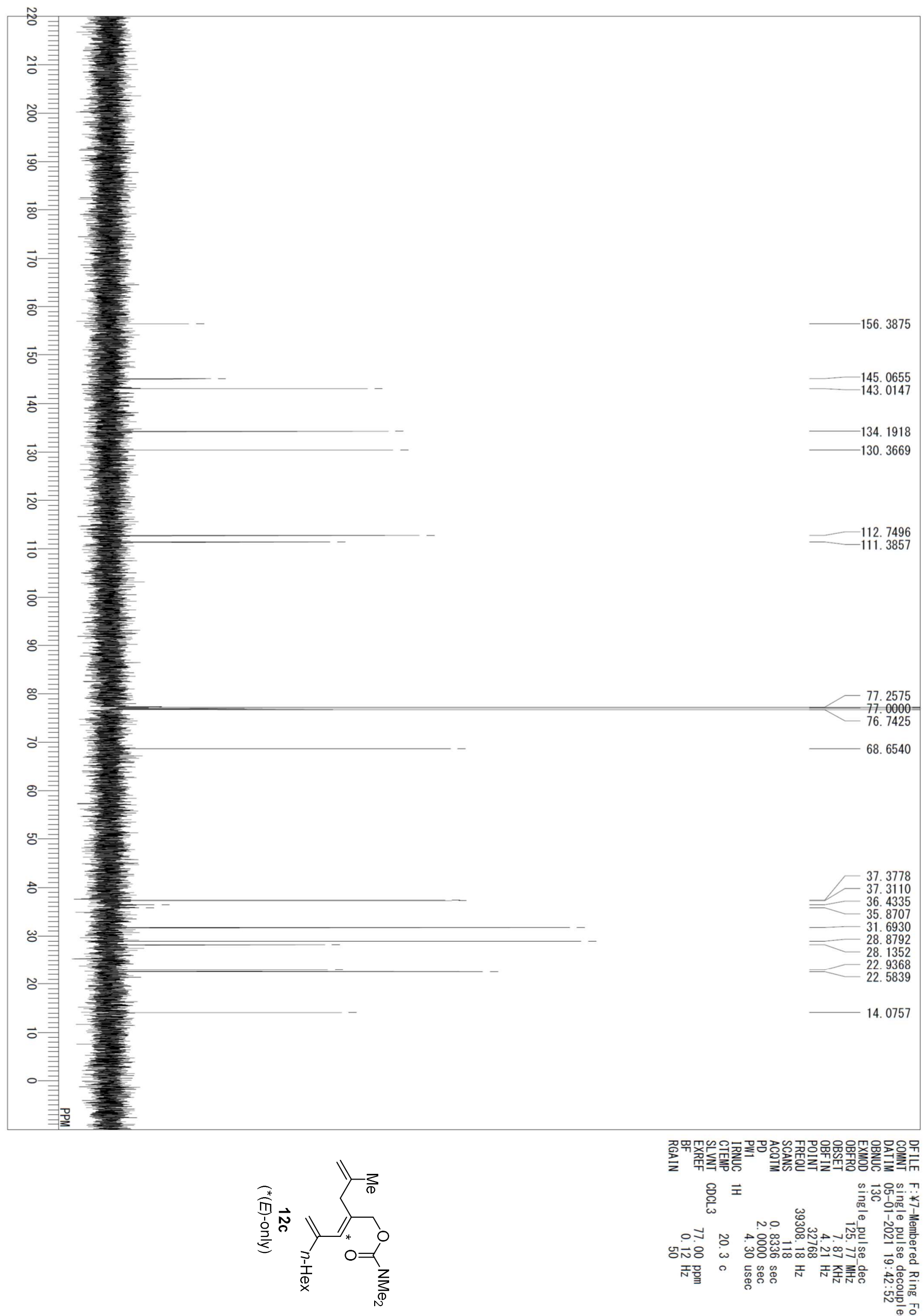

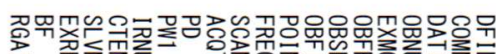

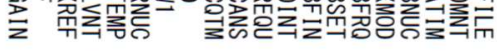

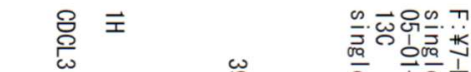

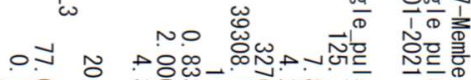

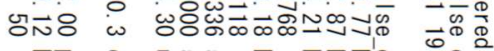

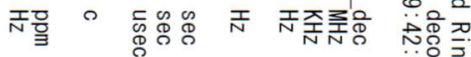

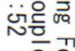



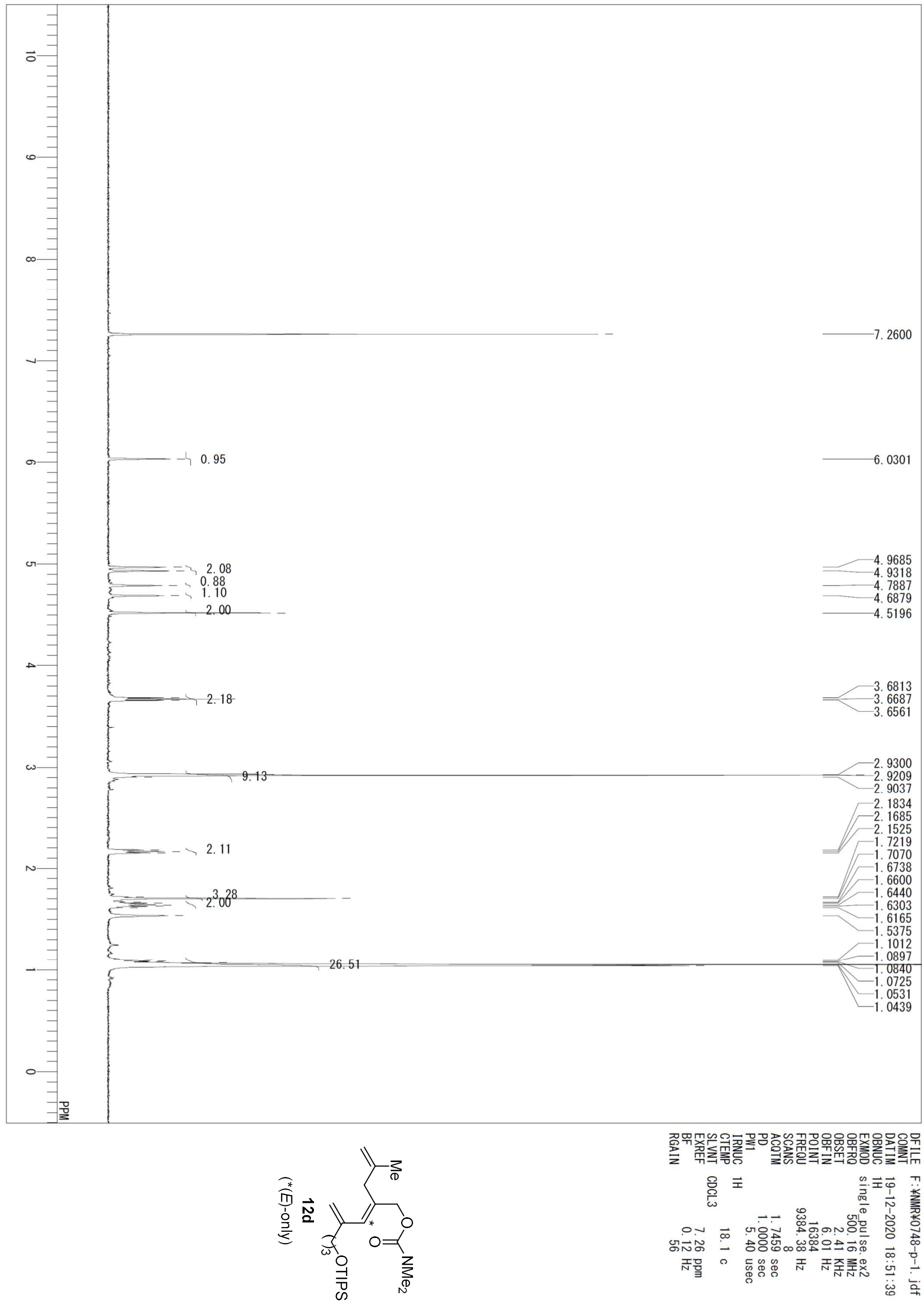

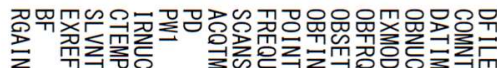

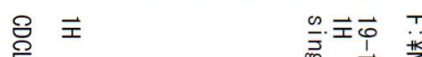

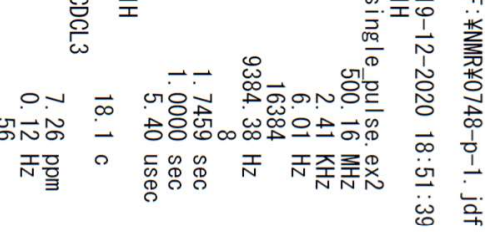



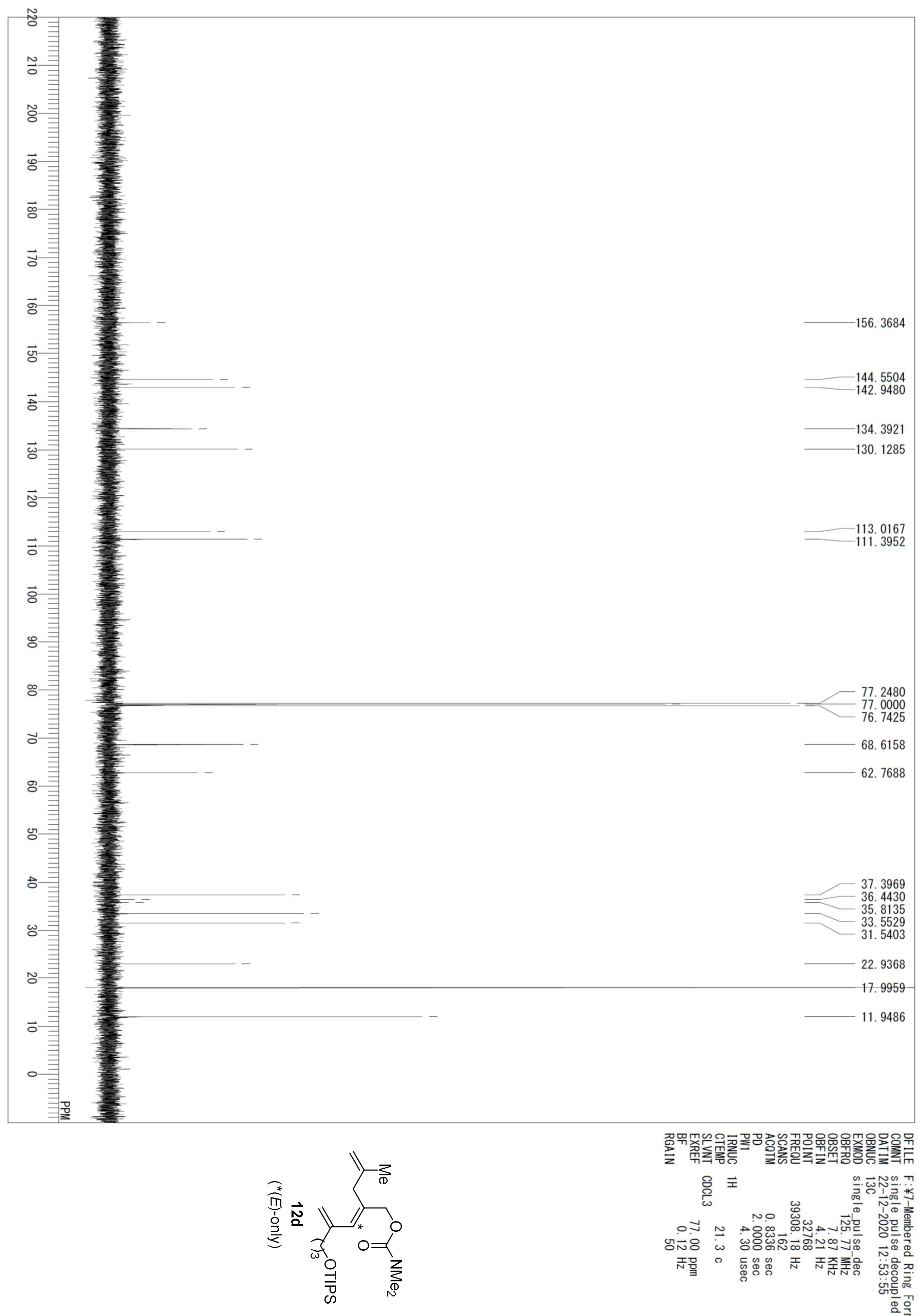

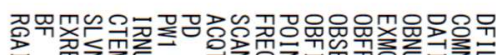

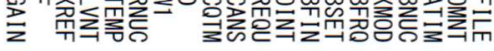

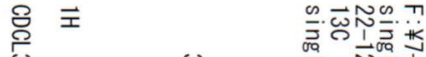

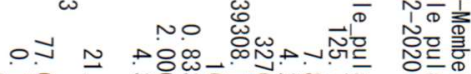

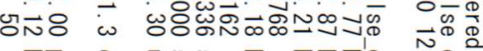

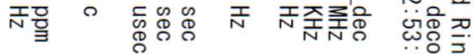

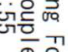



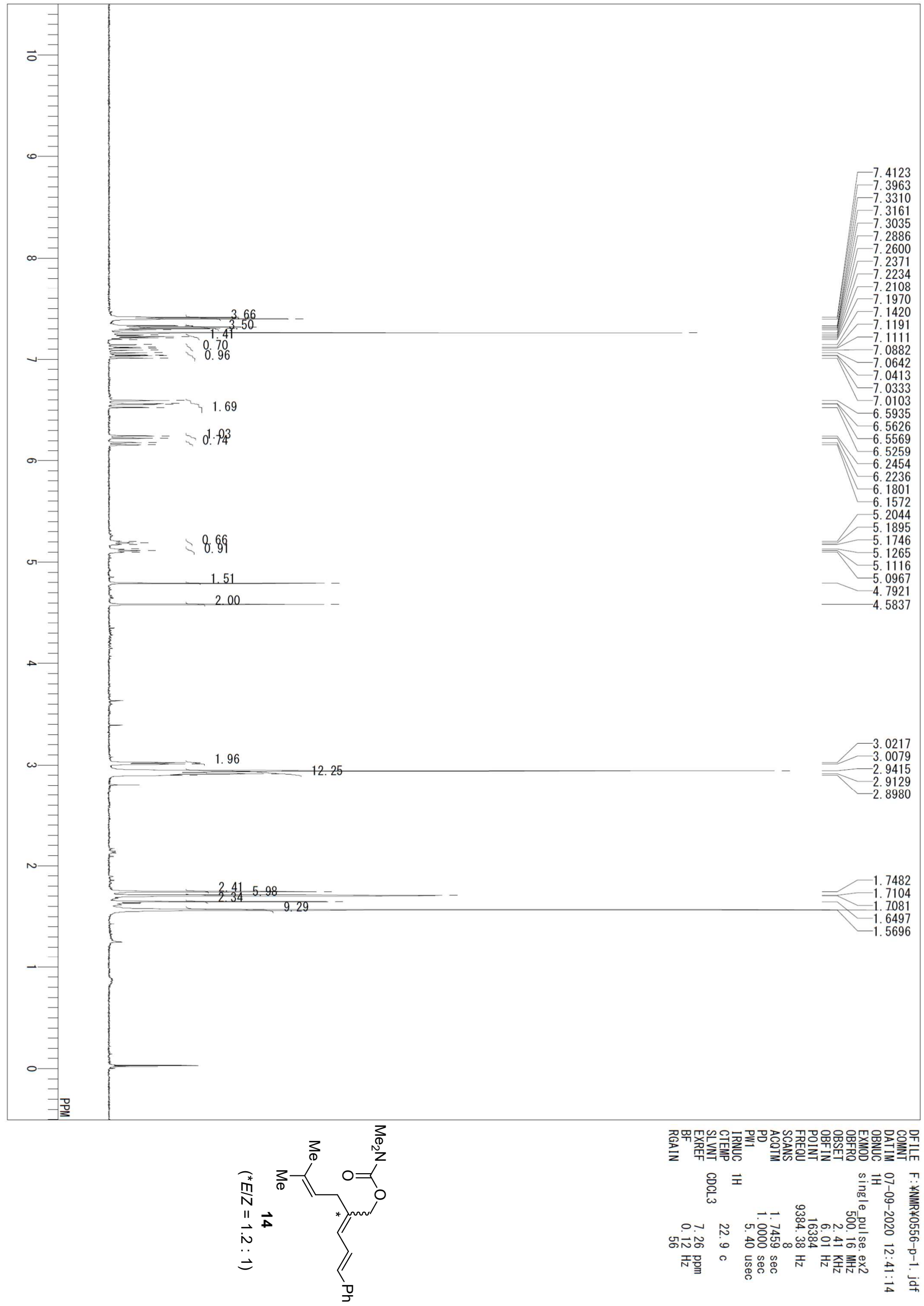

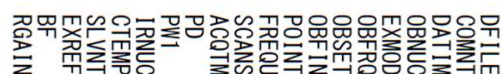

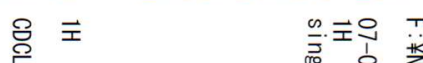

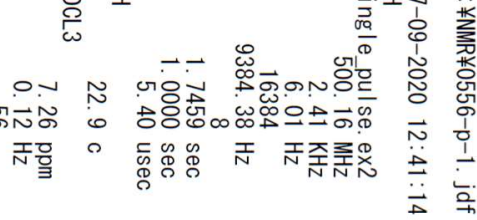




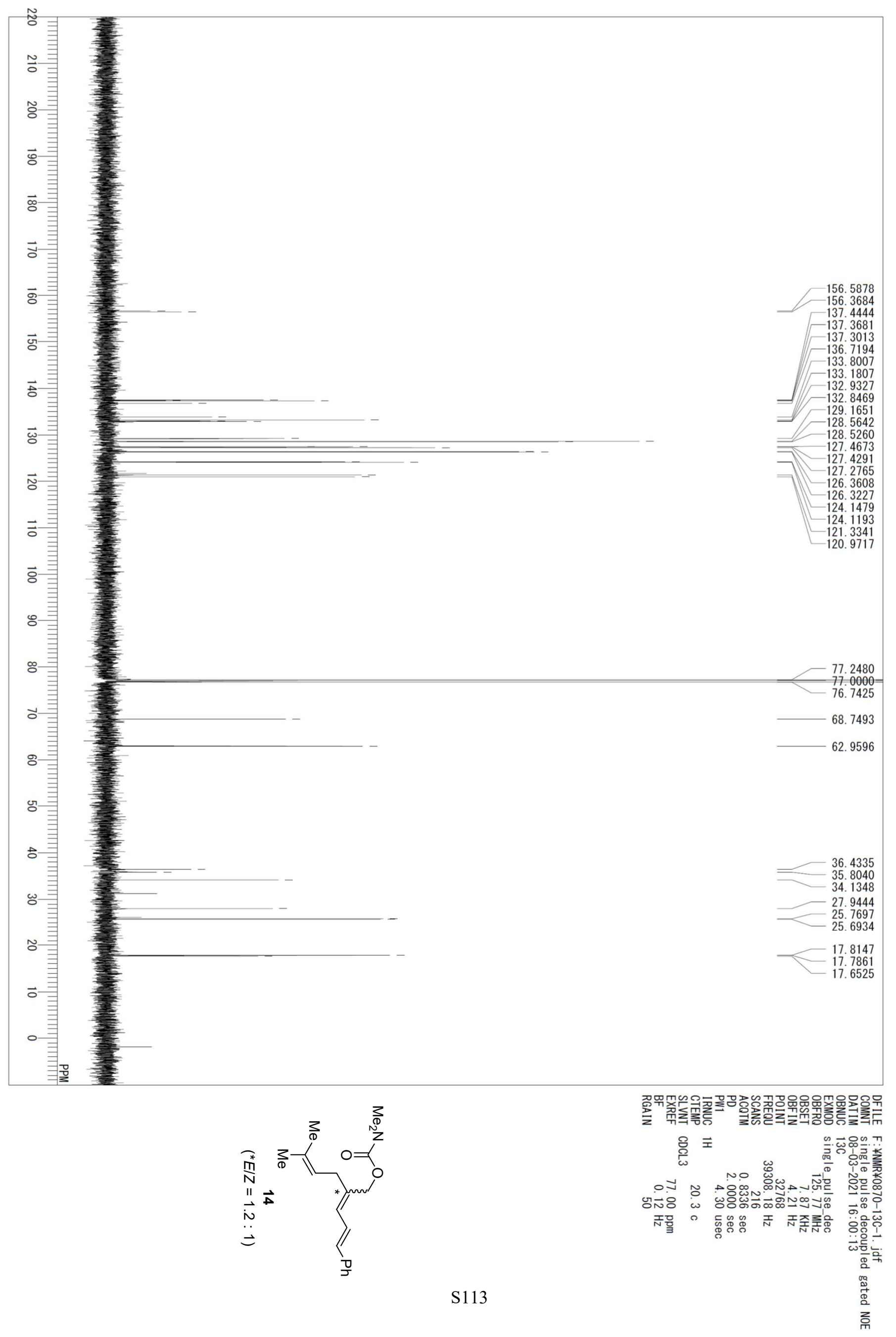




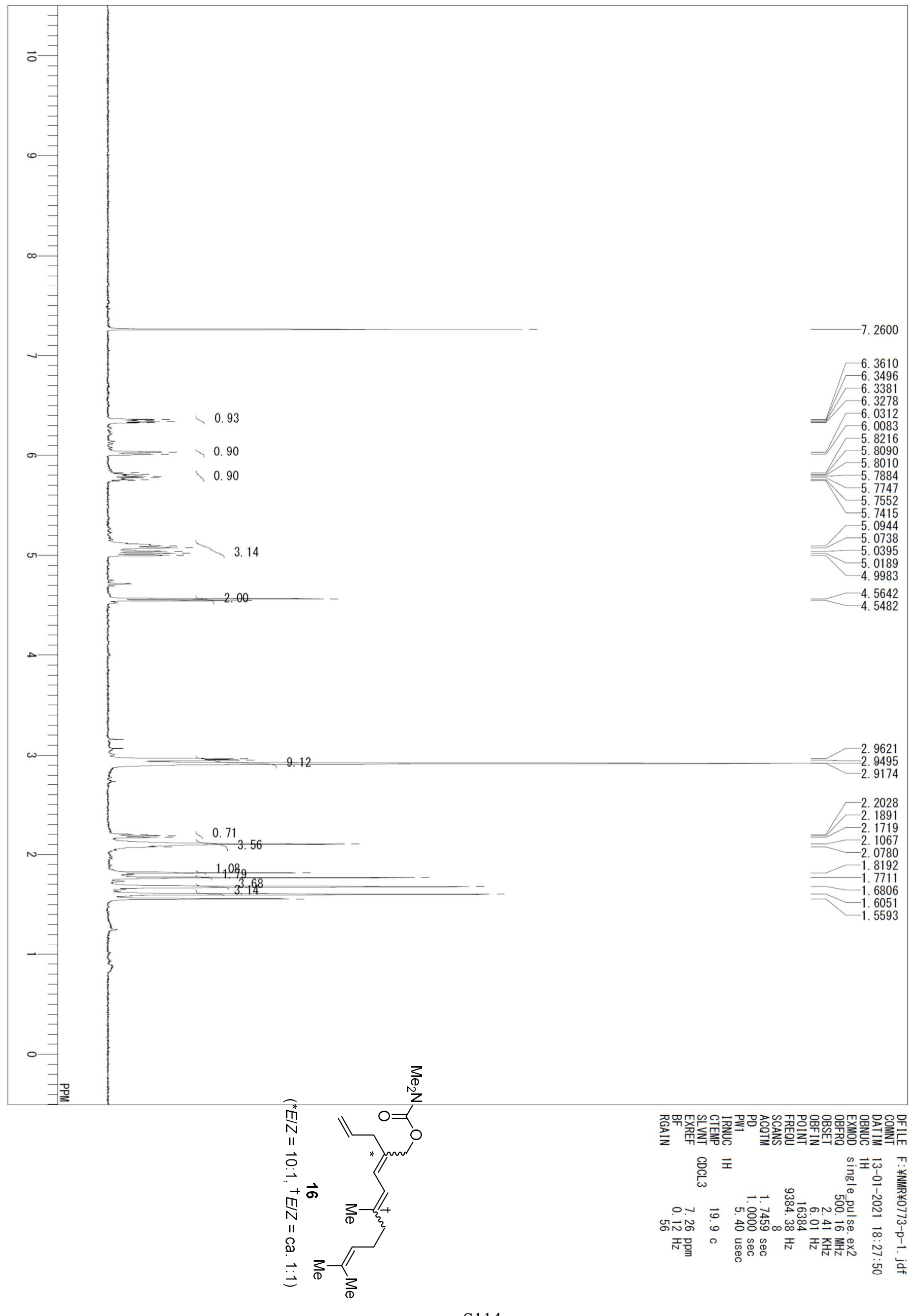




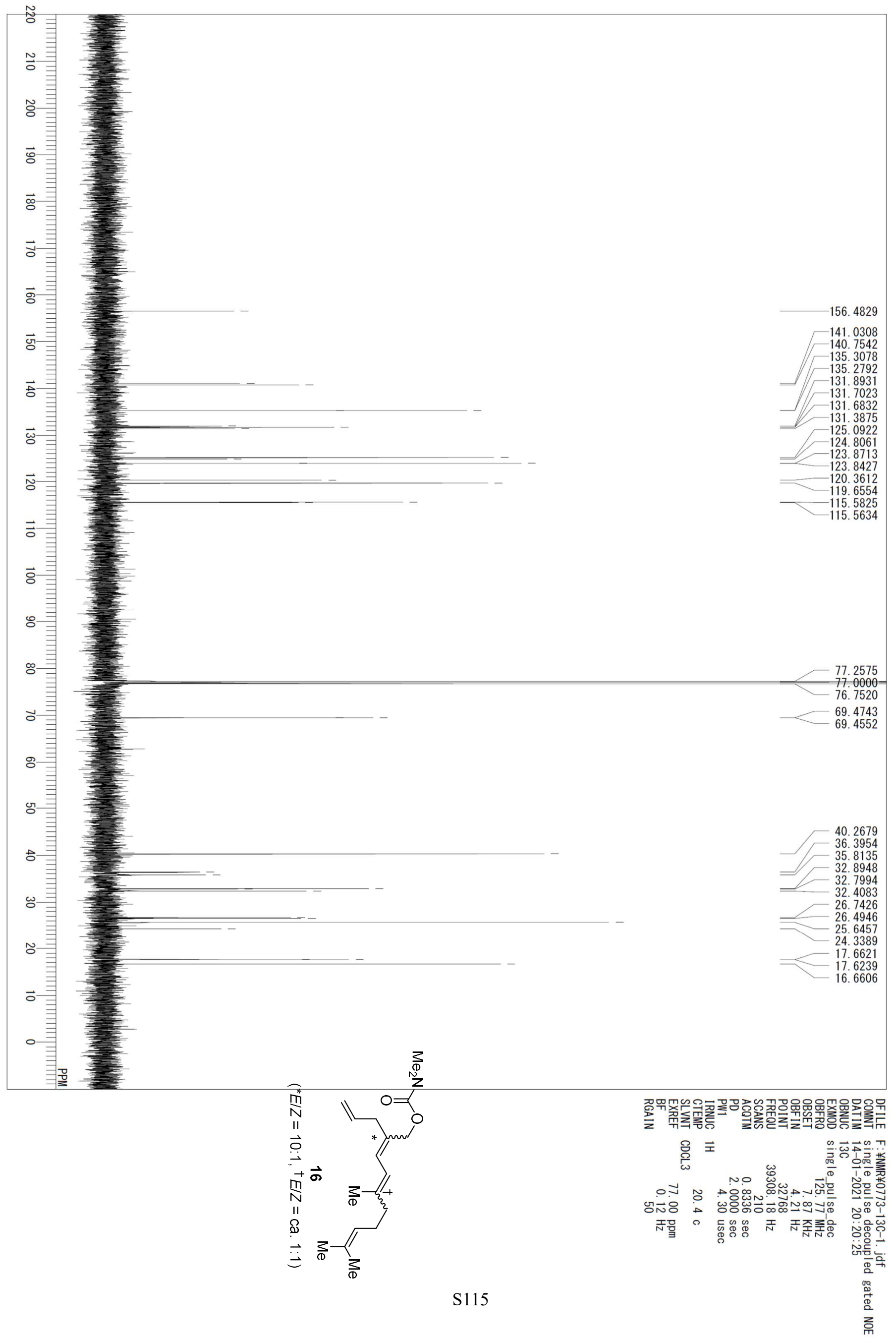



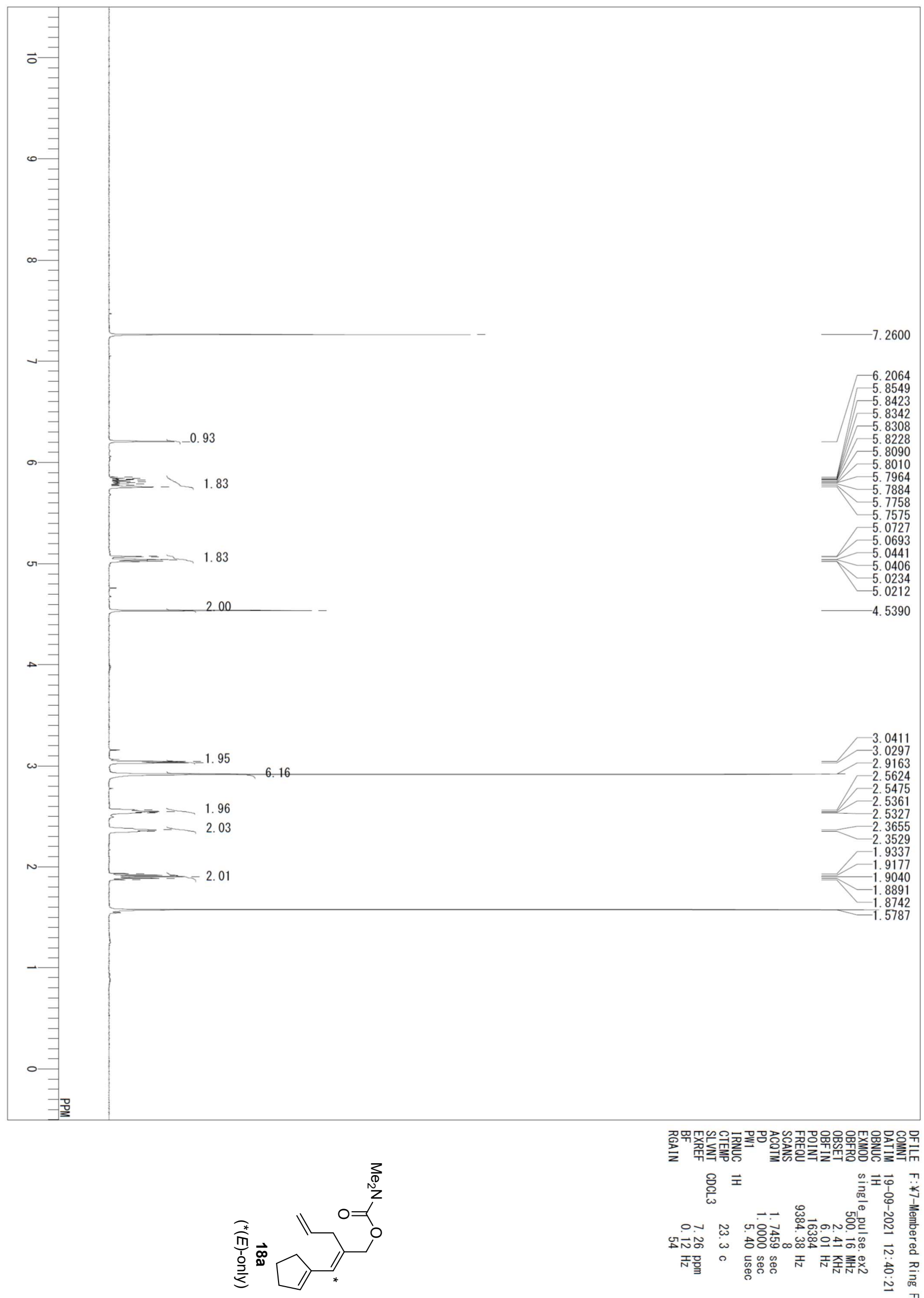

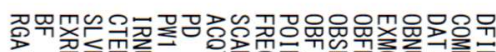

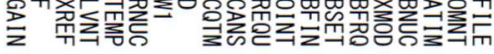

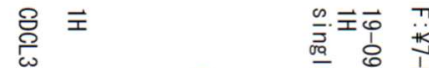

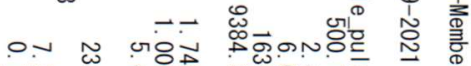
जिते

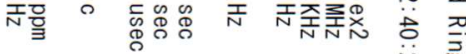



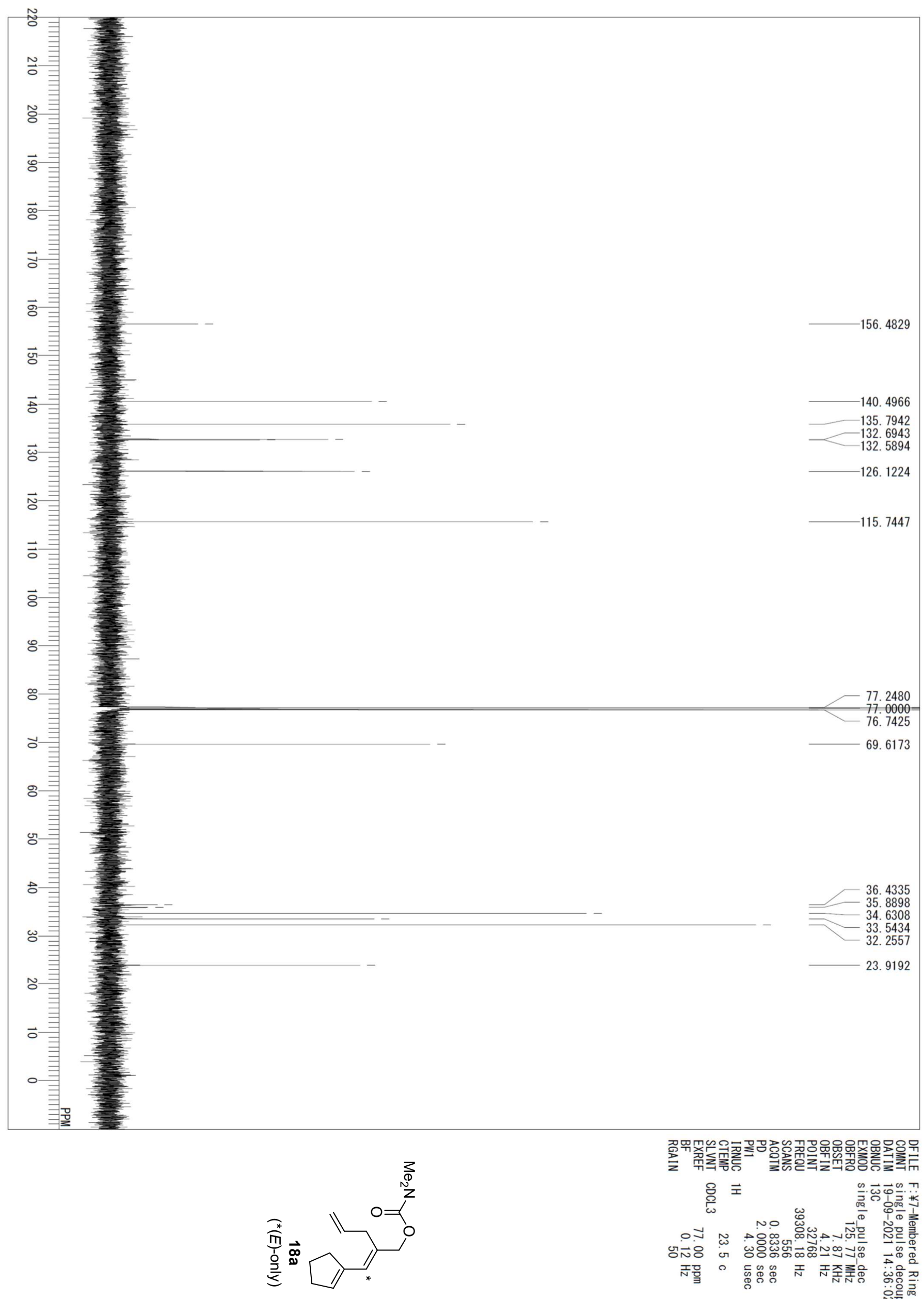

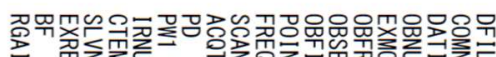

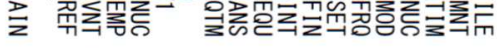

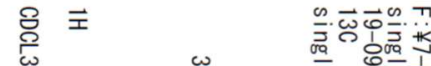

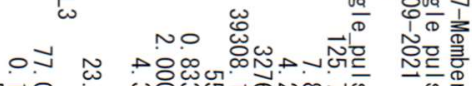

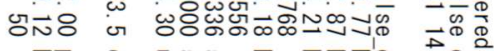

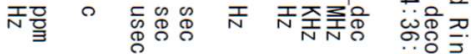




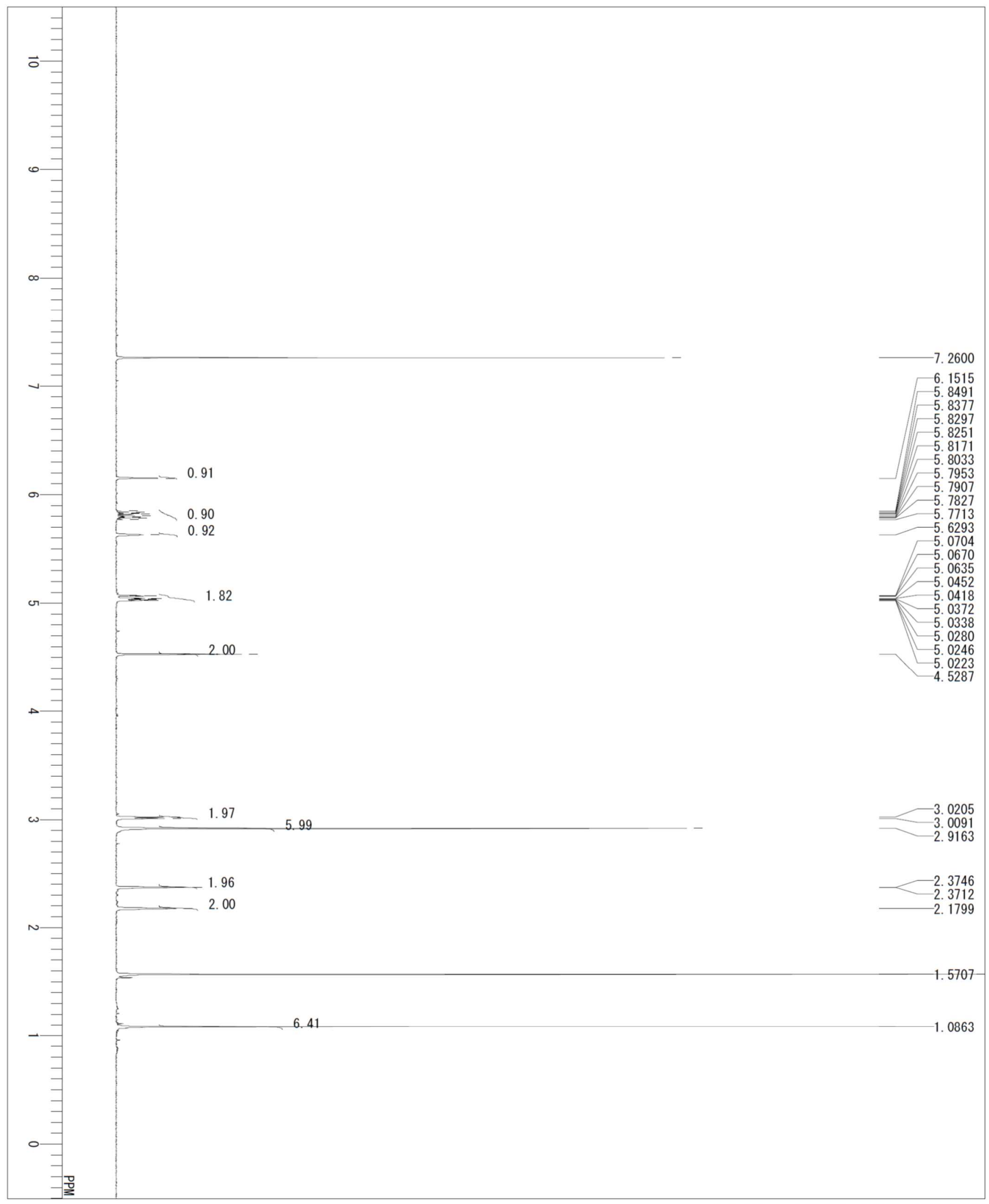

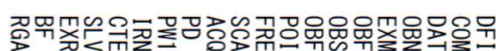

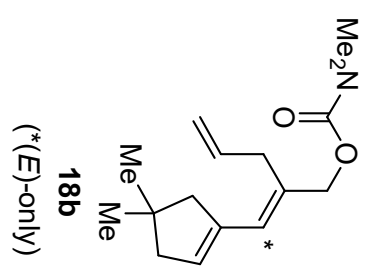

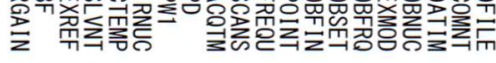

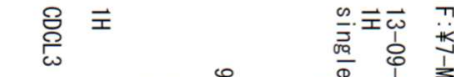

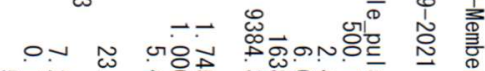
जंत̄

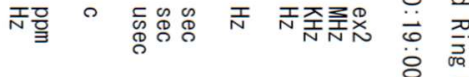



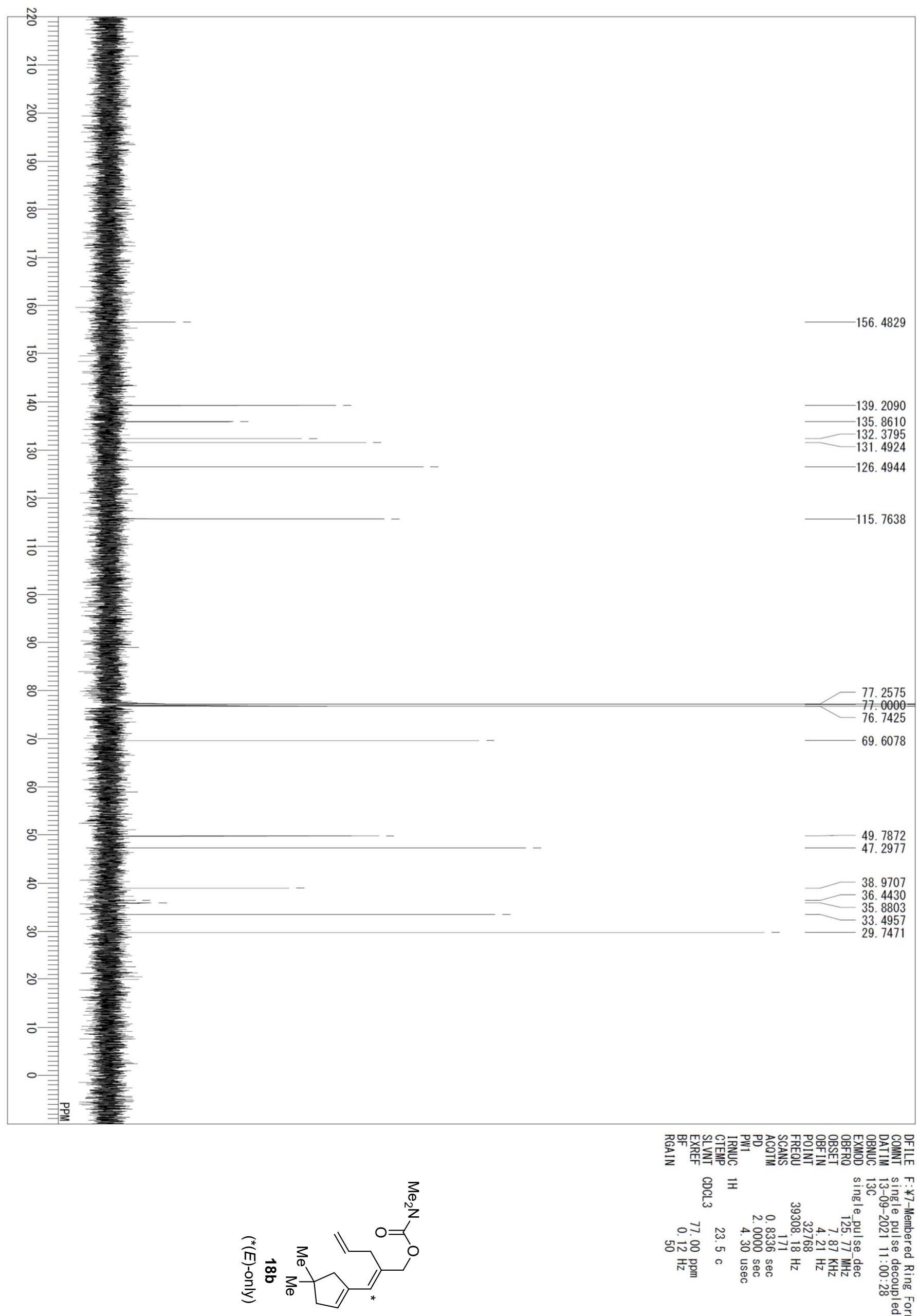

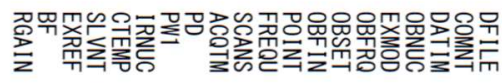

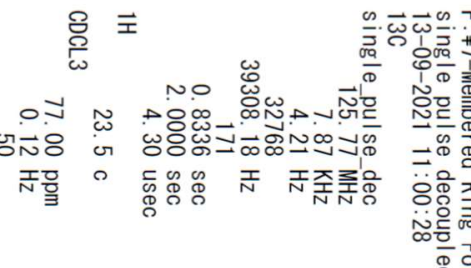



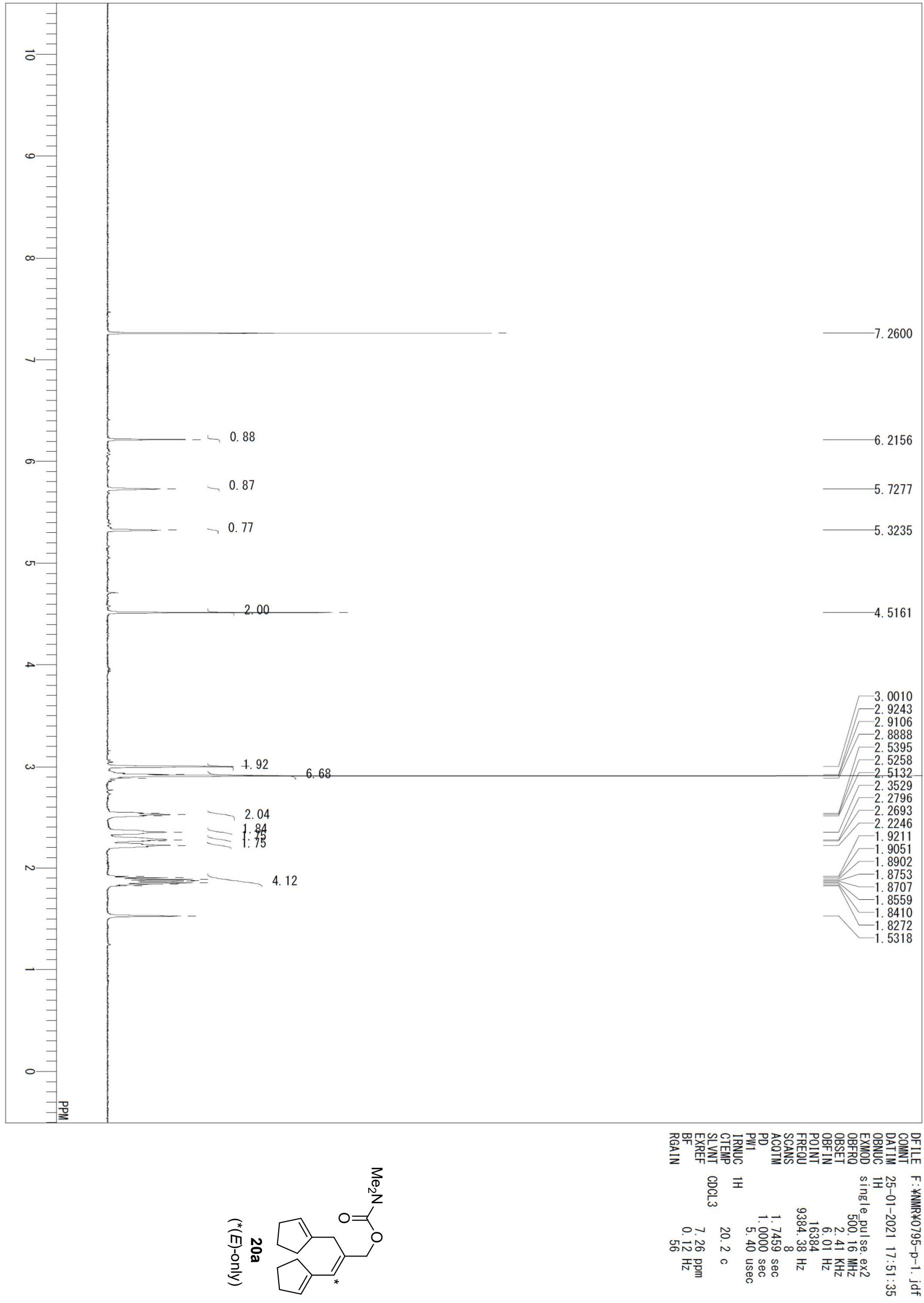

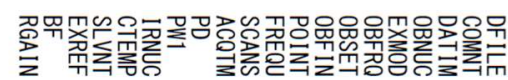

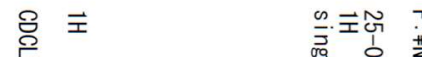

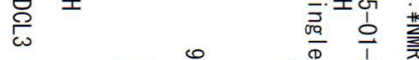

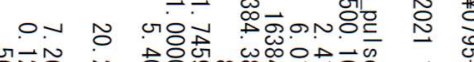

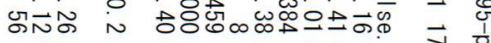

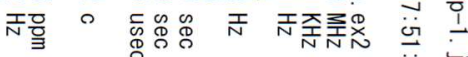
菂 

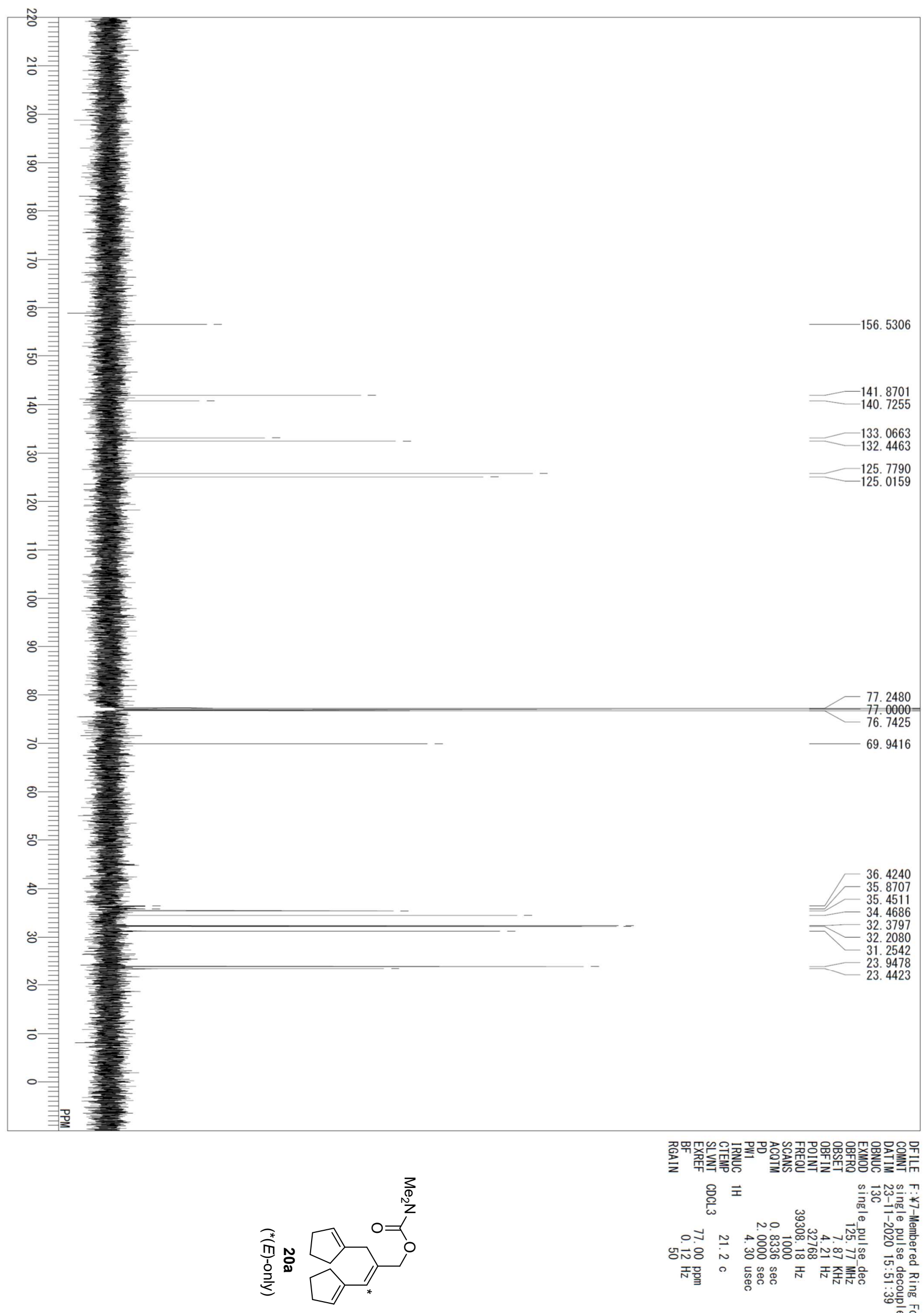

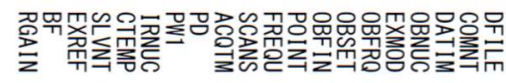

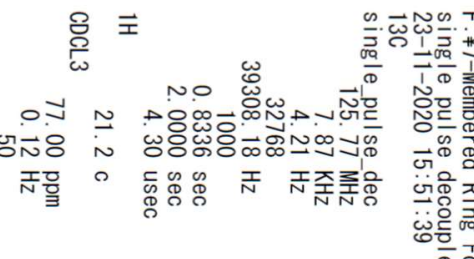



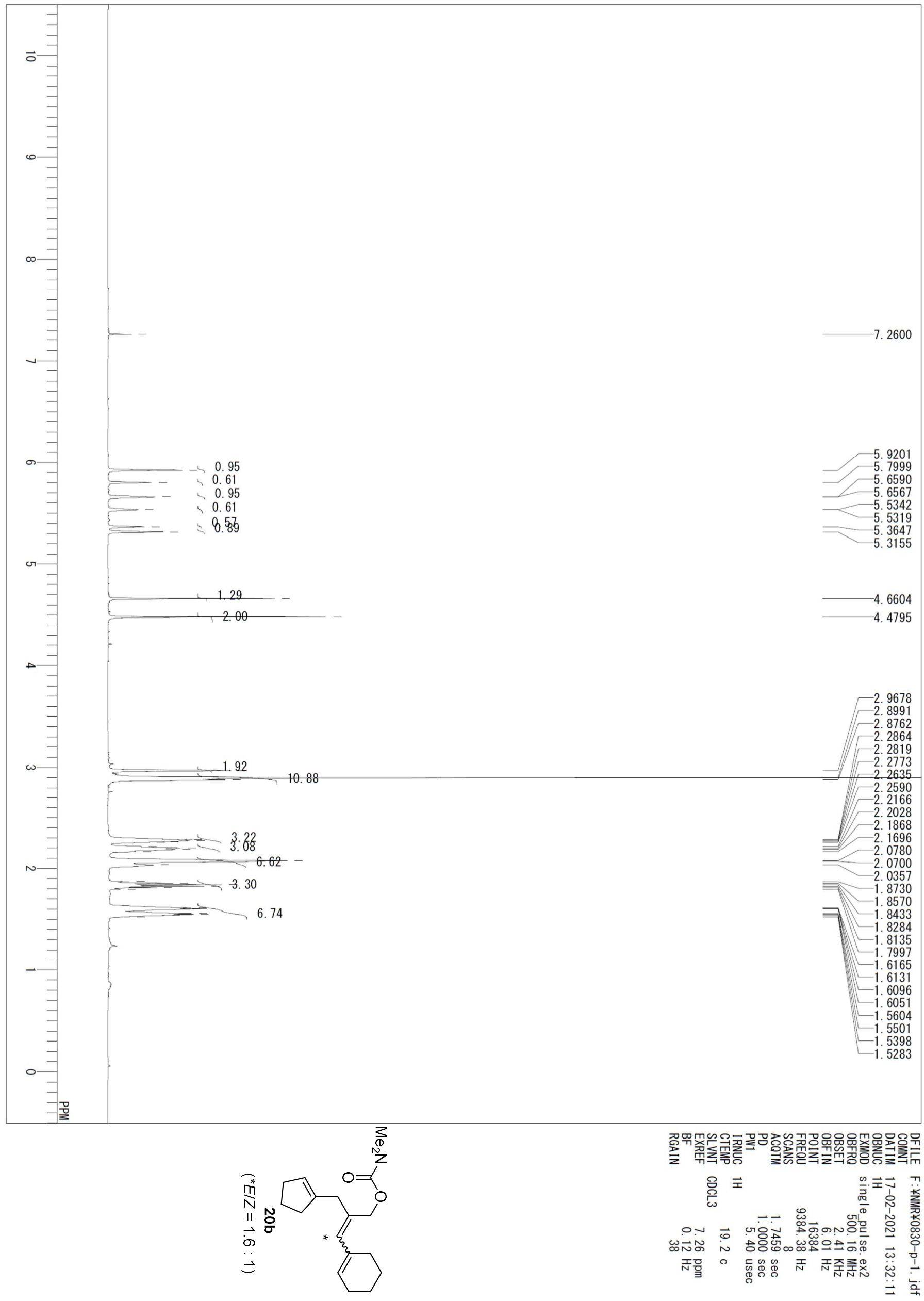

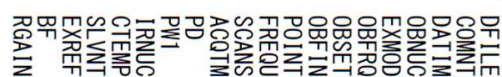

总

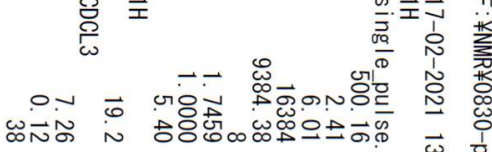

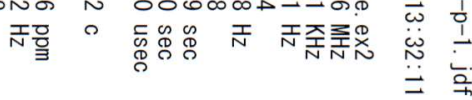




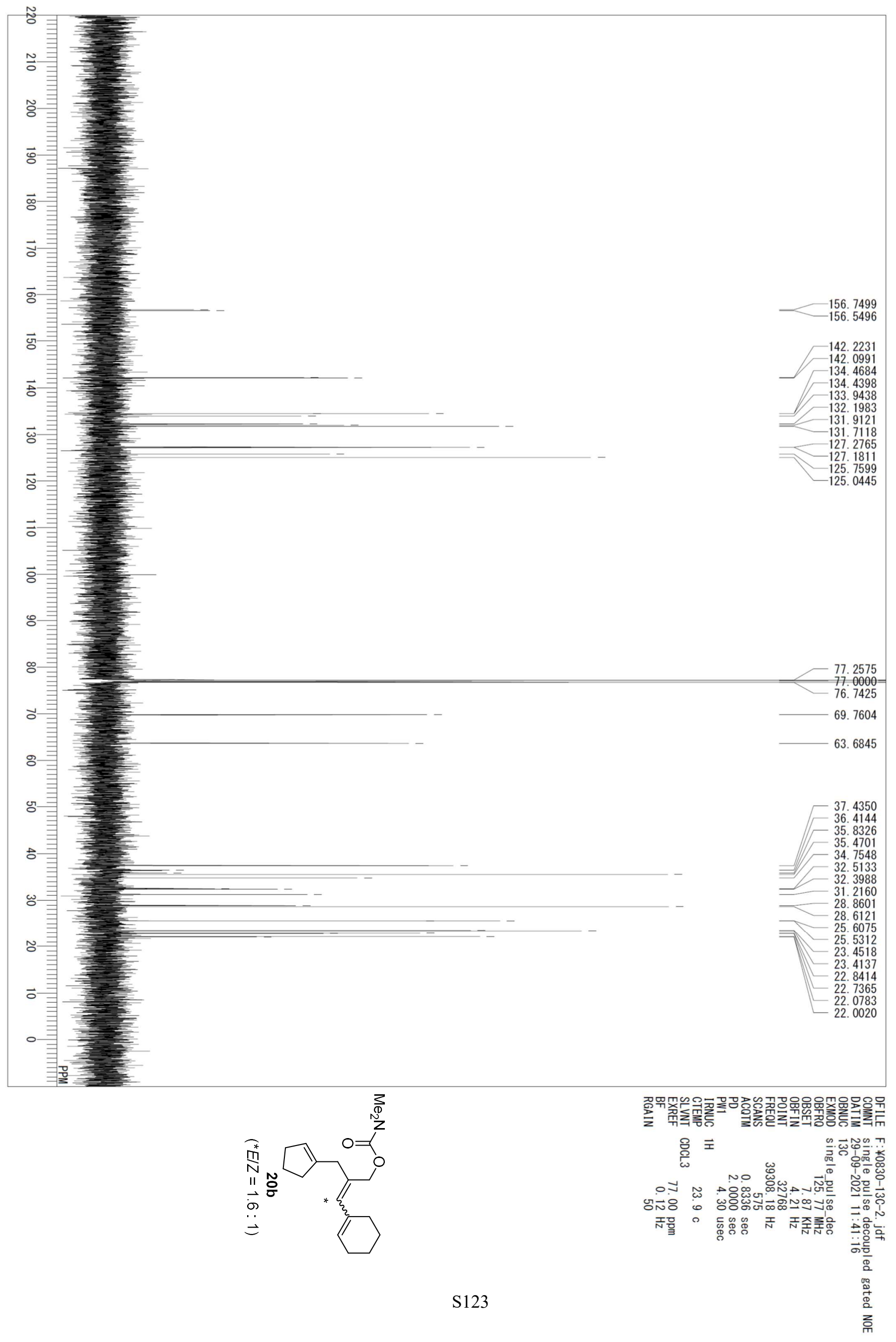



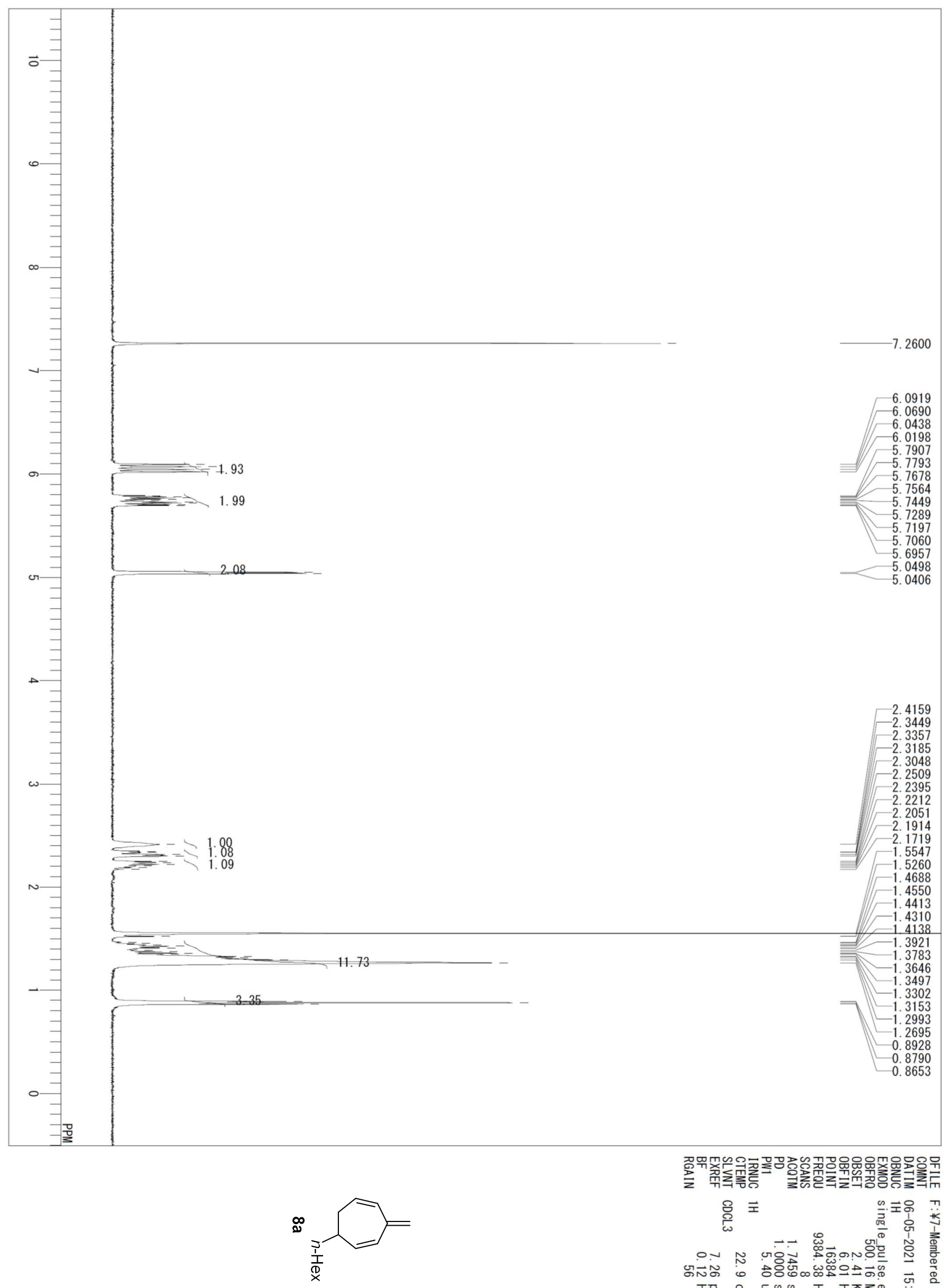

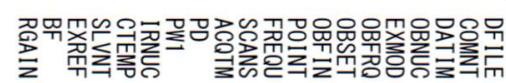

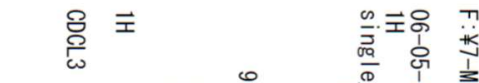
o. $\mathrm{N}$ Mion जुत̄

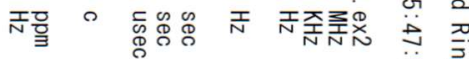




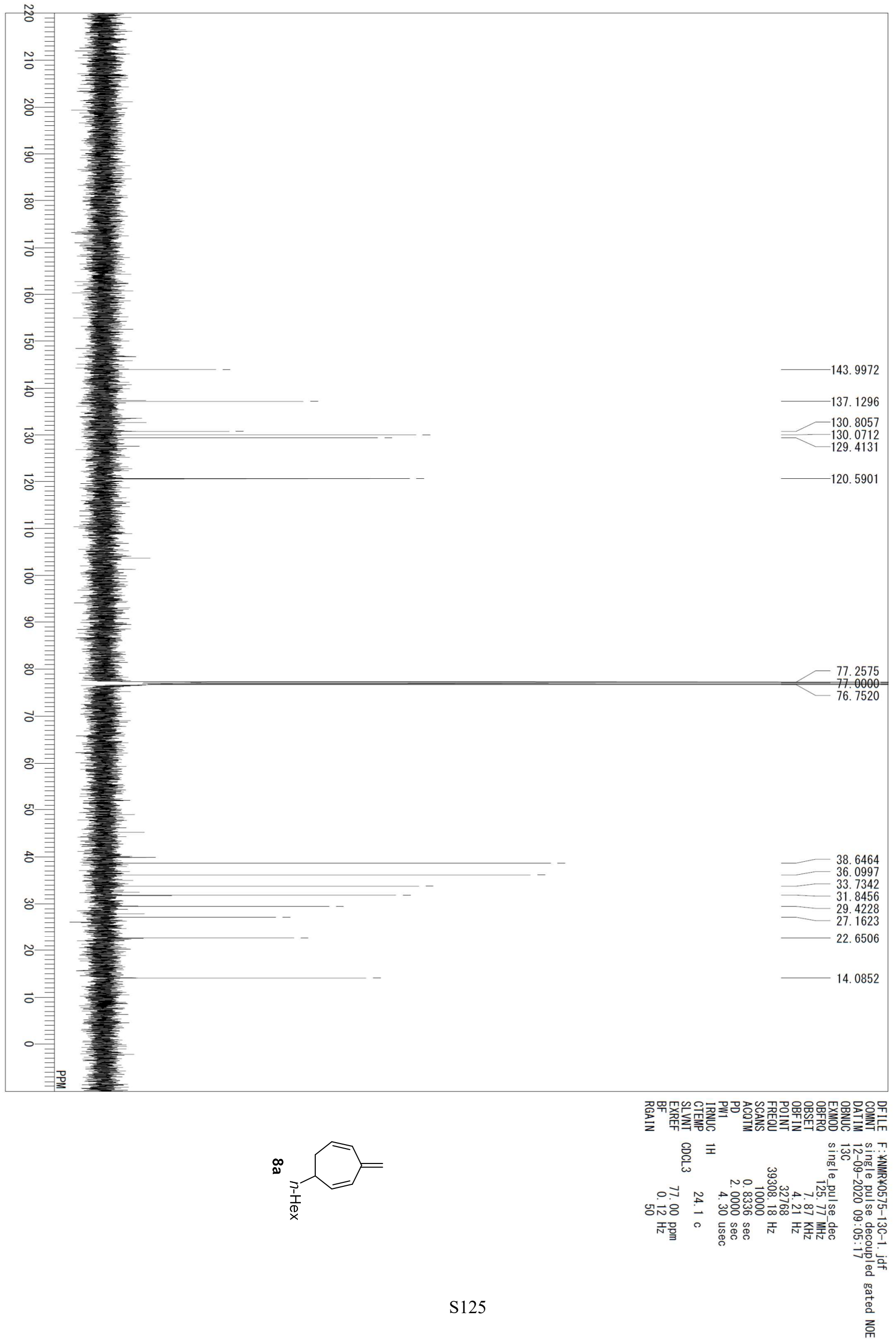




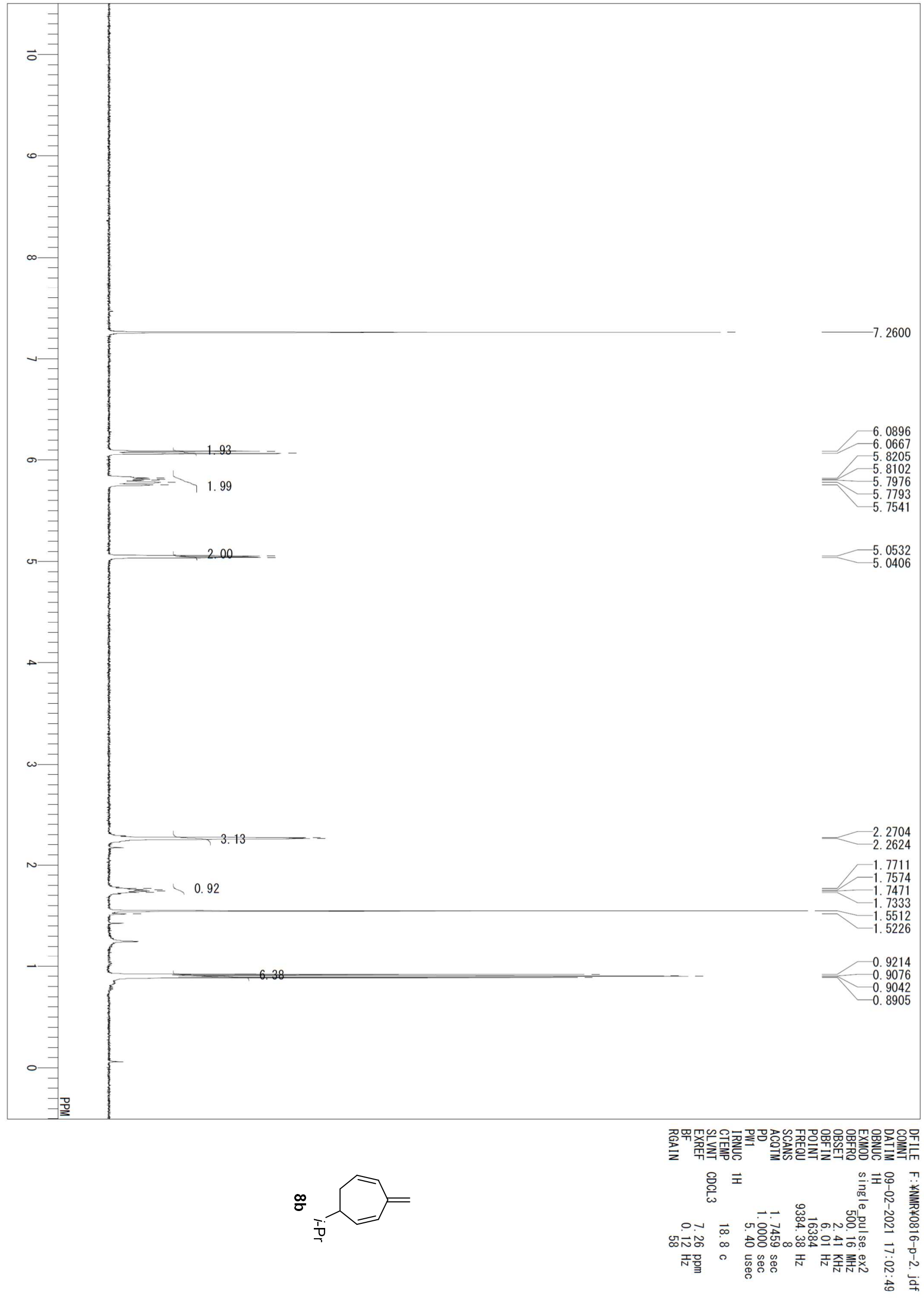




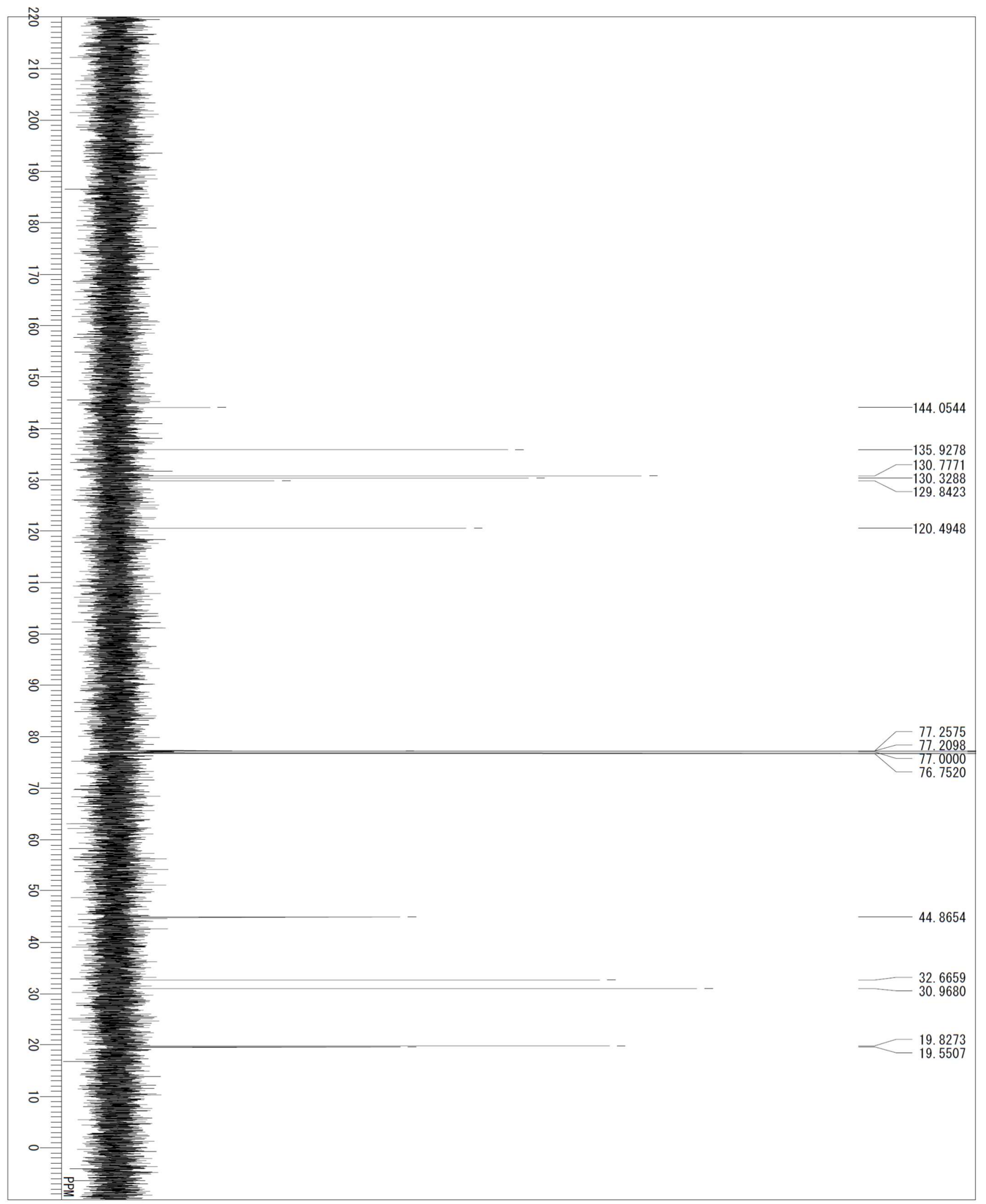

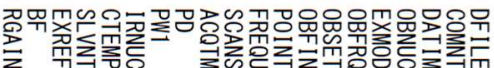

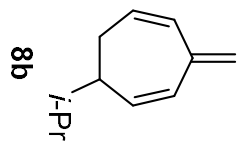

욤표

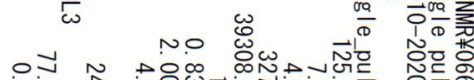

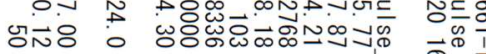

점믈 


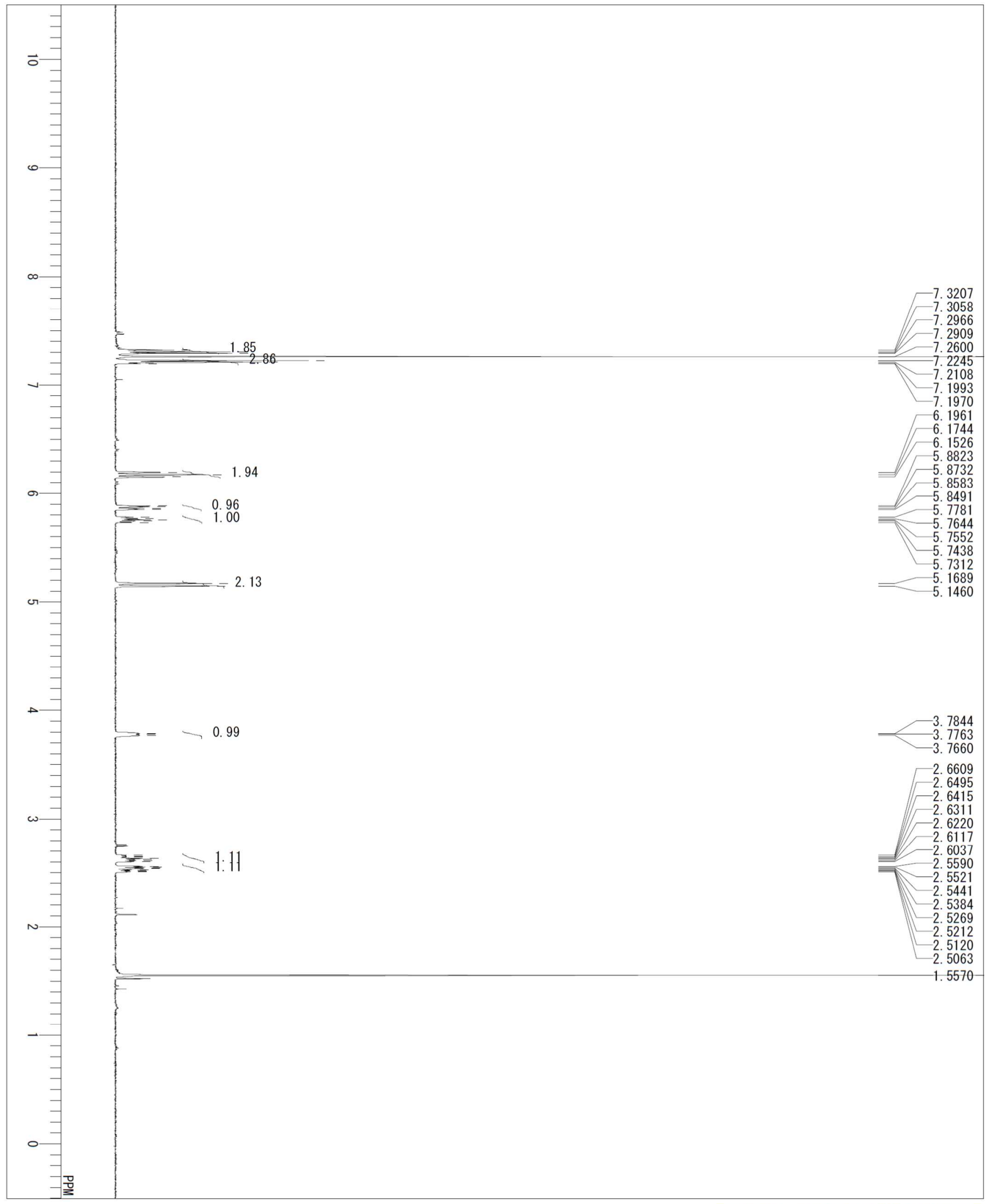

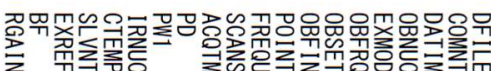

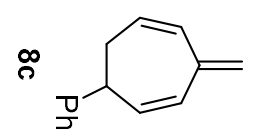

总

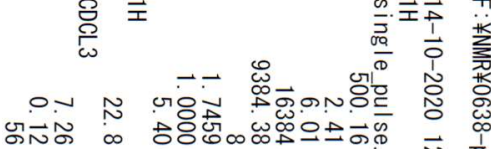

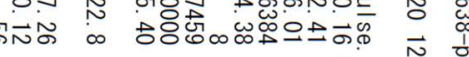

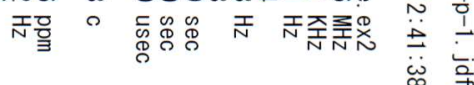



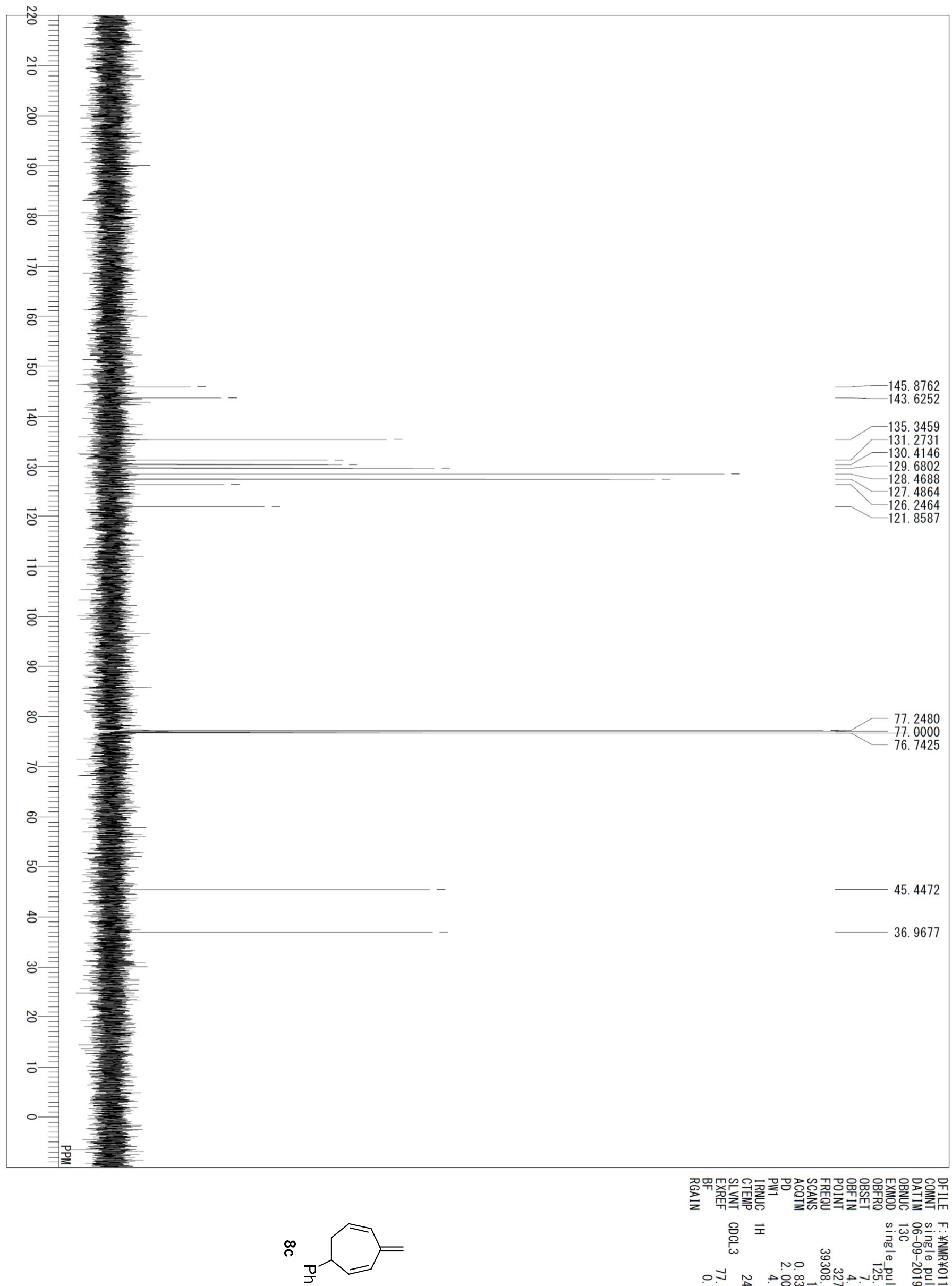

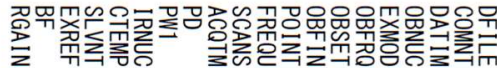

욤포

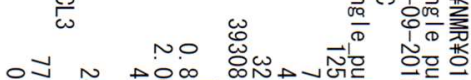

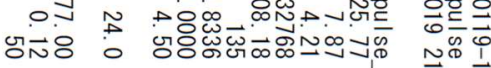

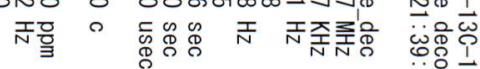

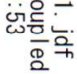



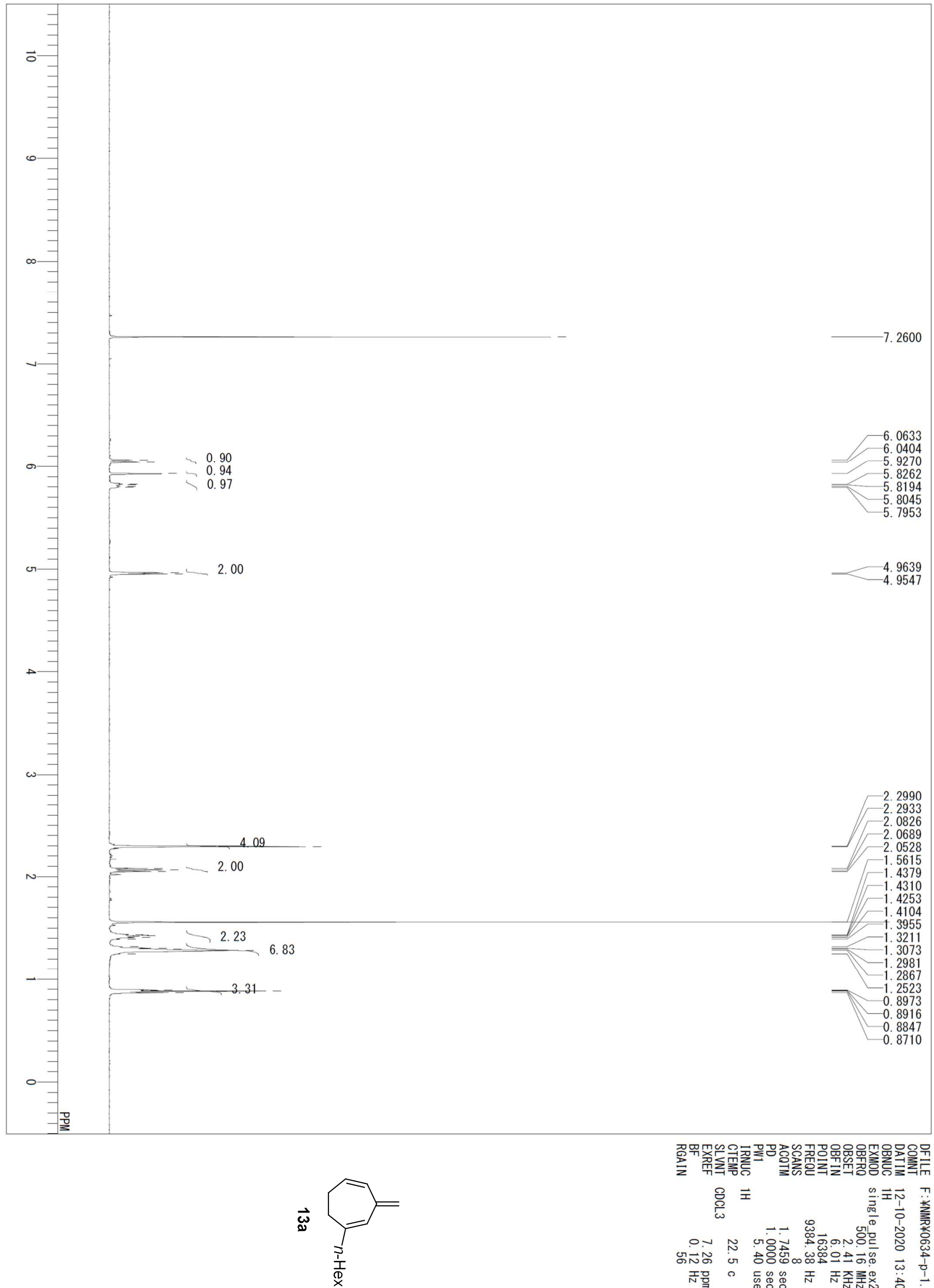

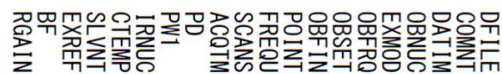

僁

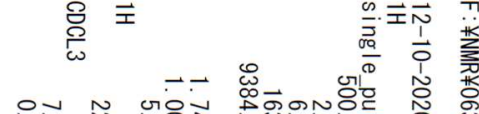

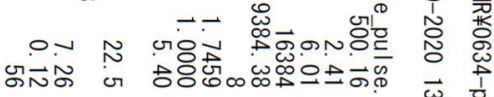

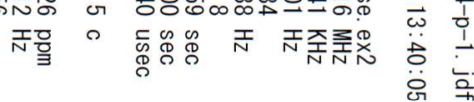



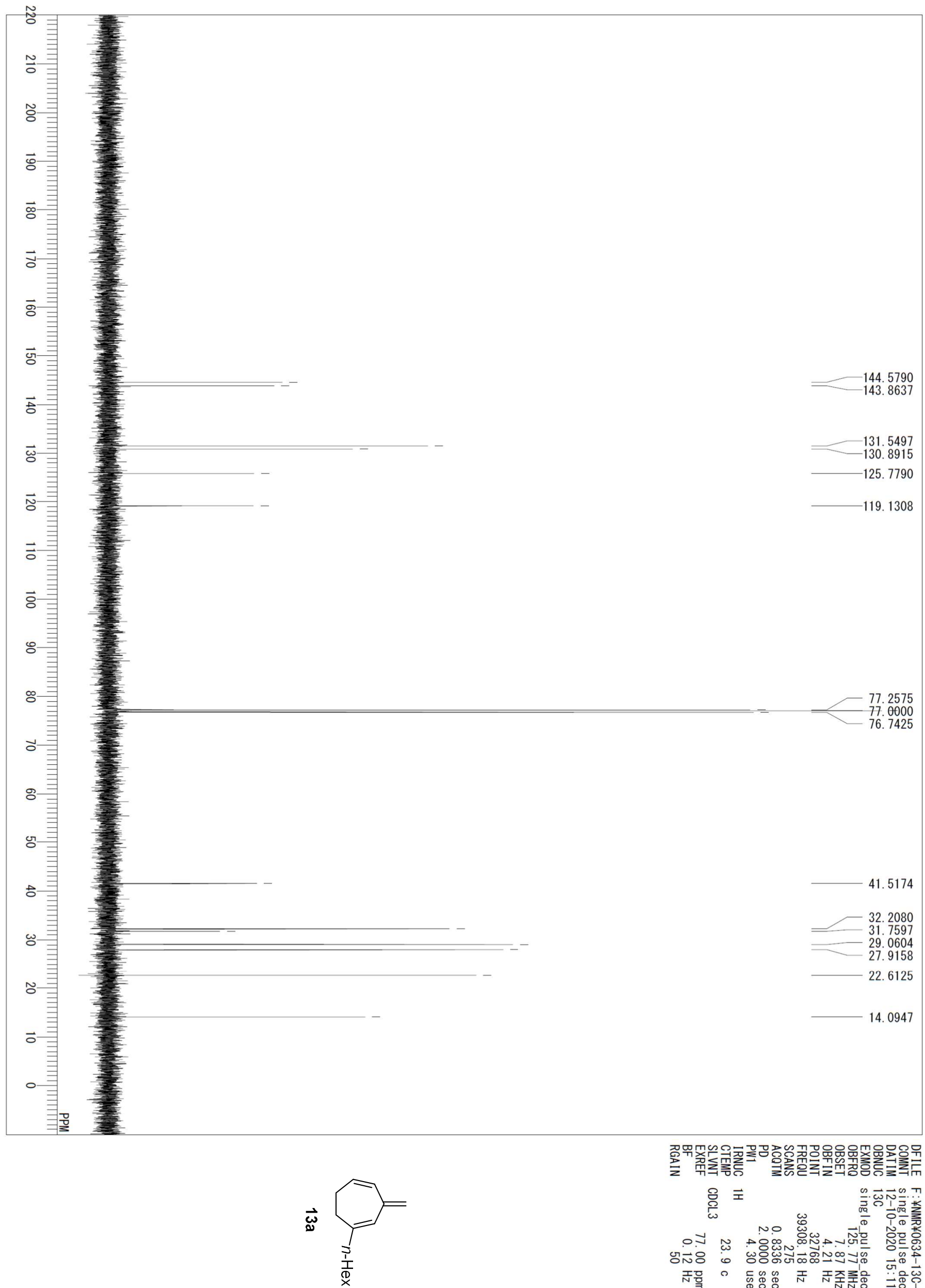

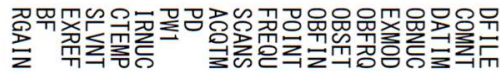
욤표

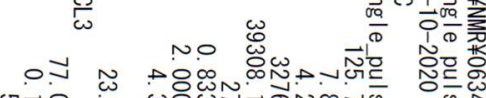

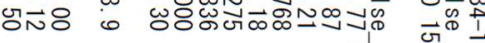

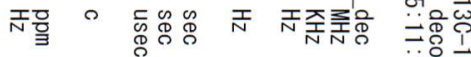

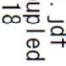



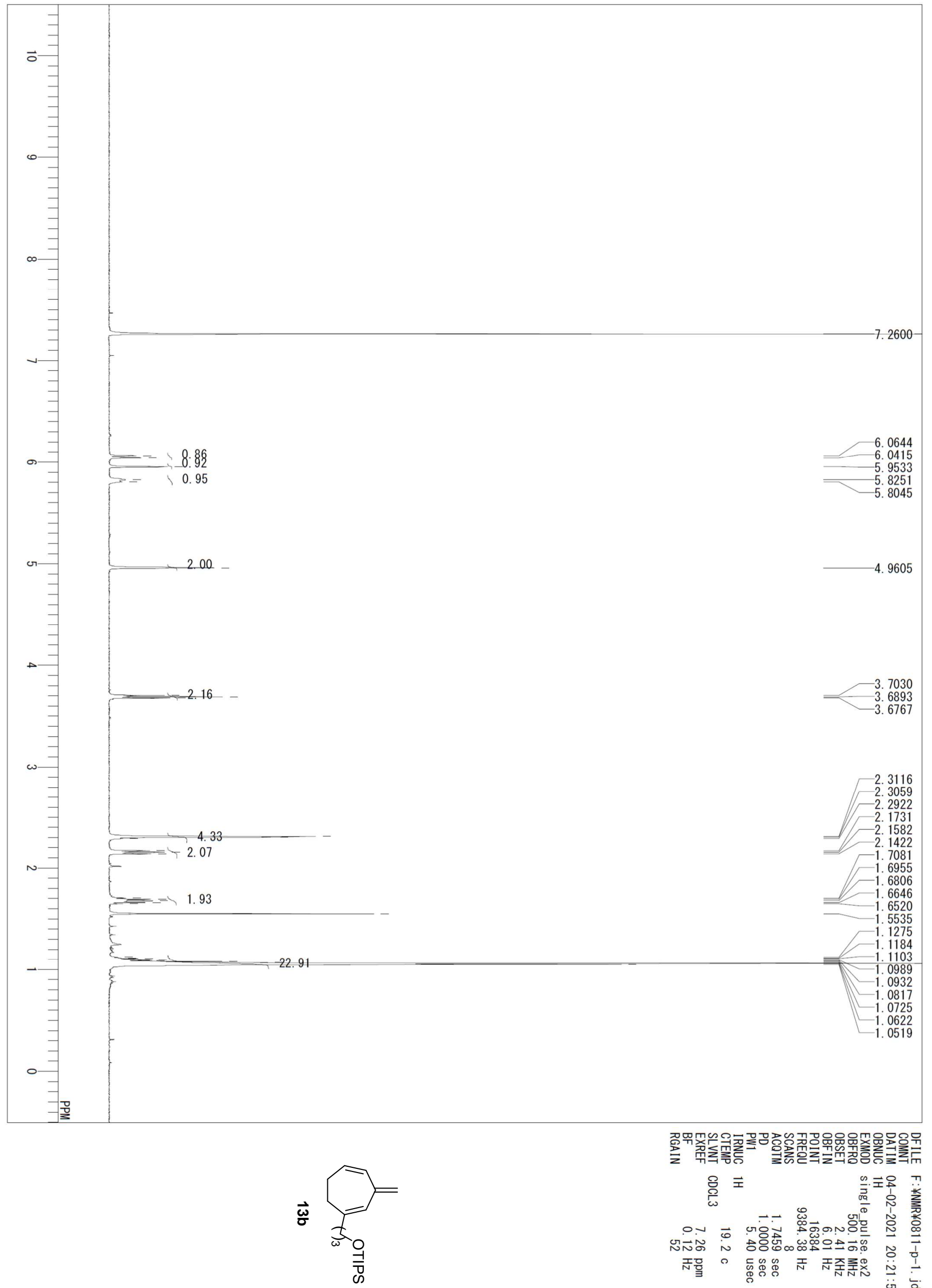

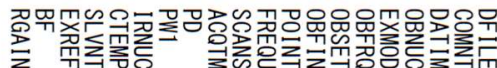

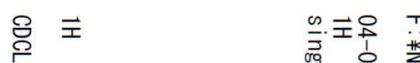

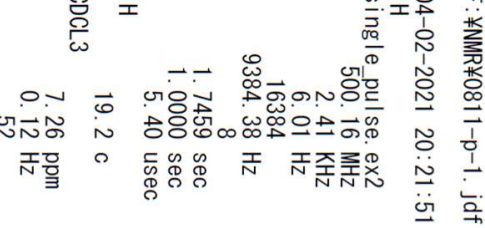




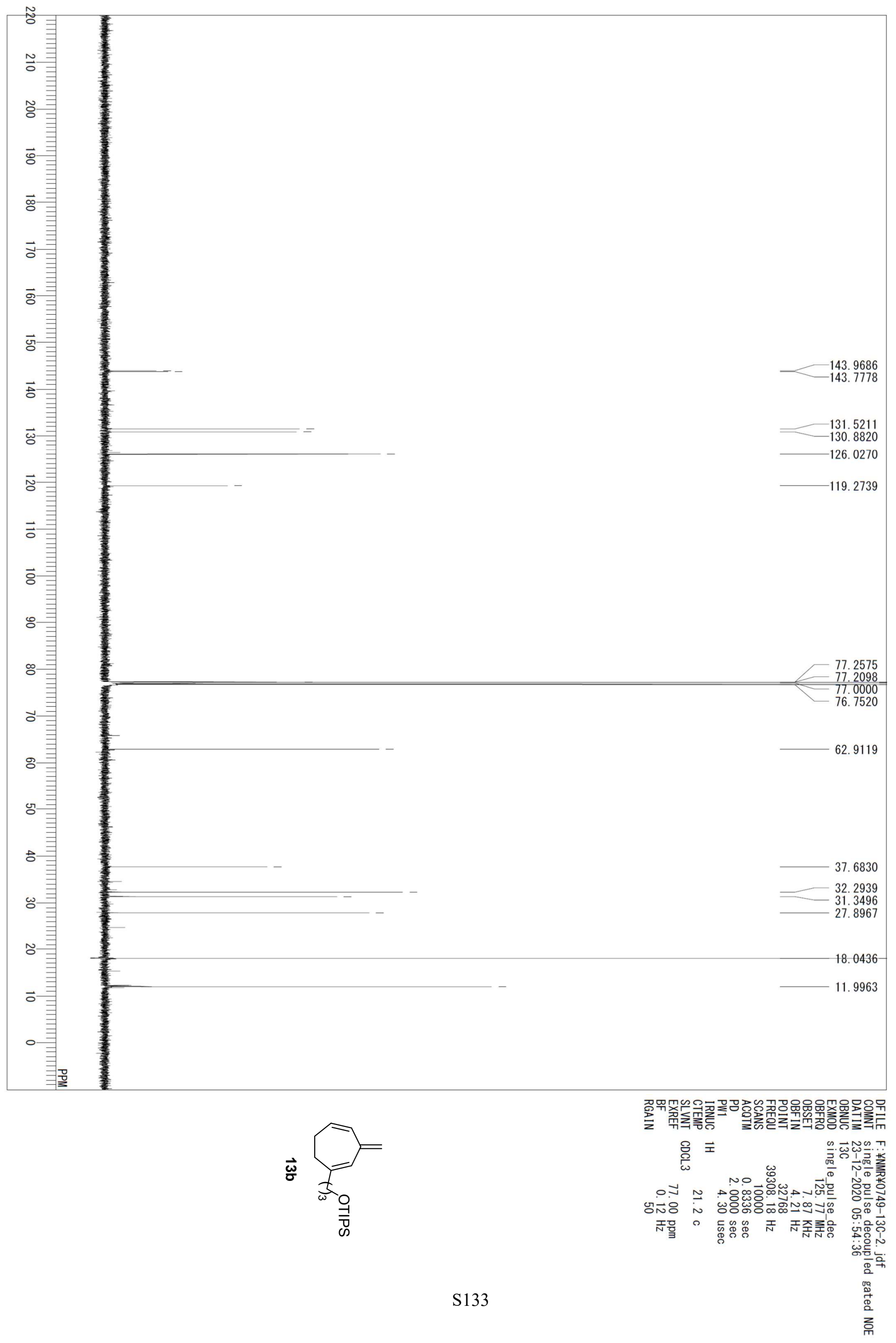




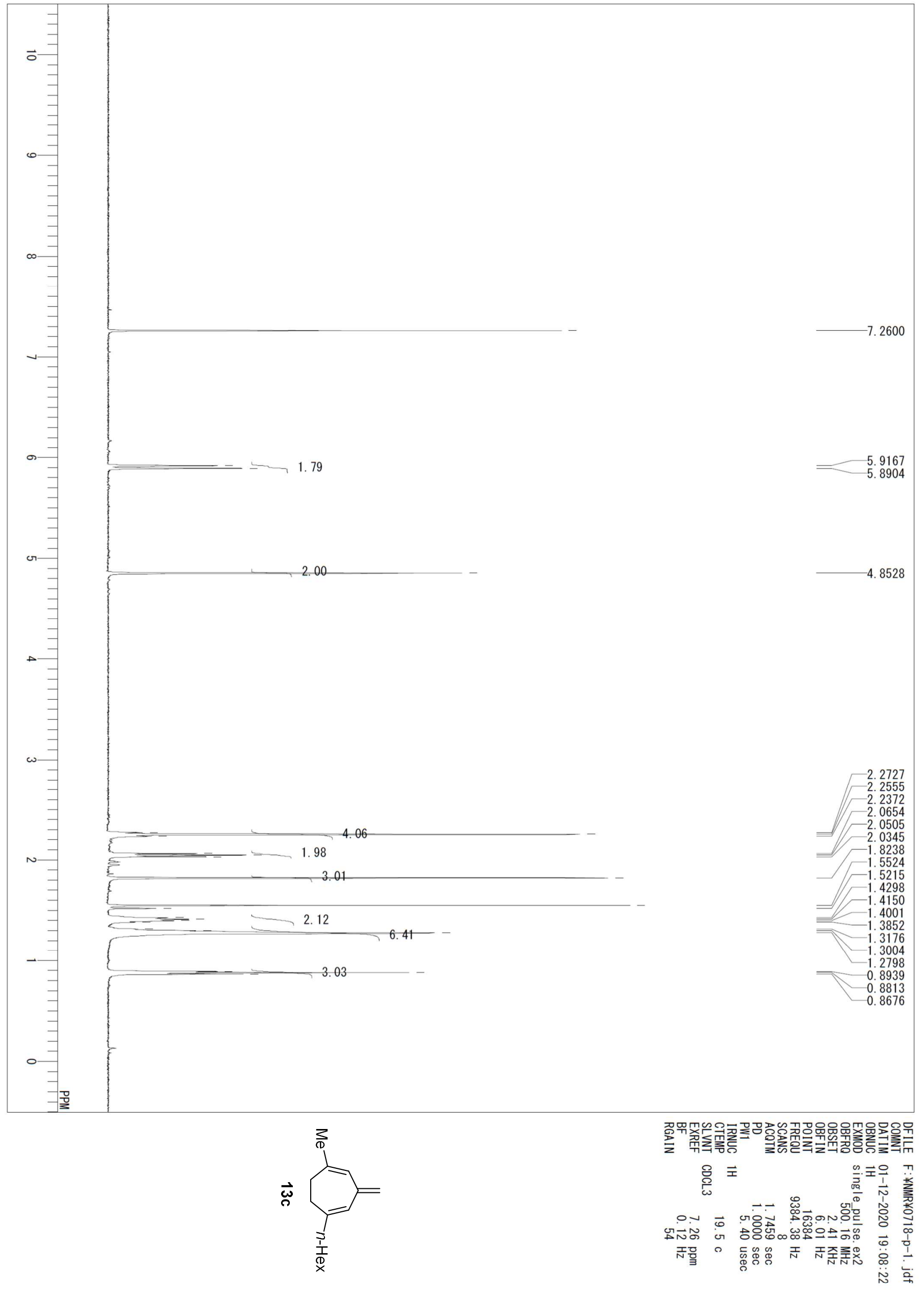



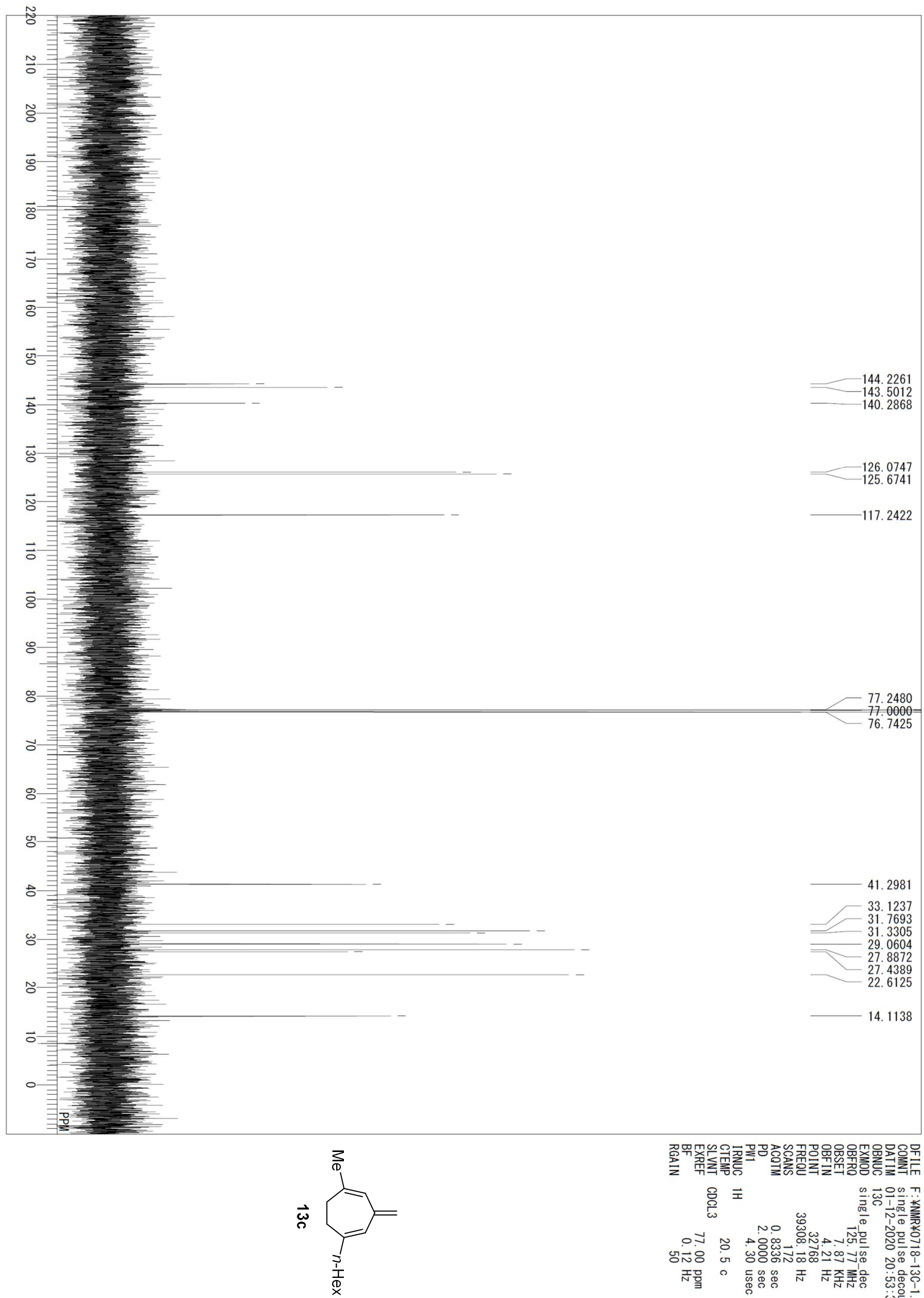

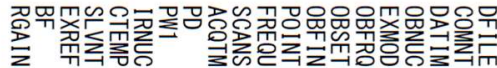

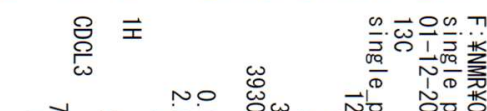

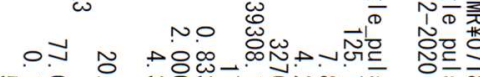

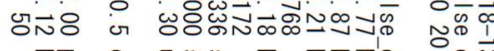

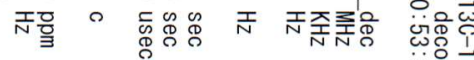



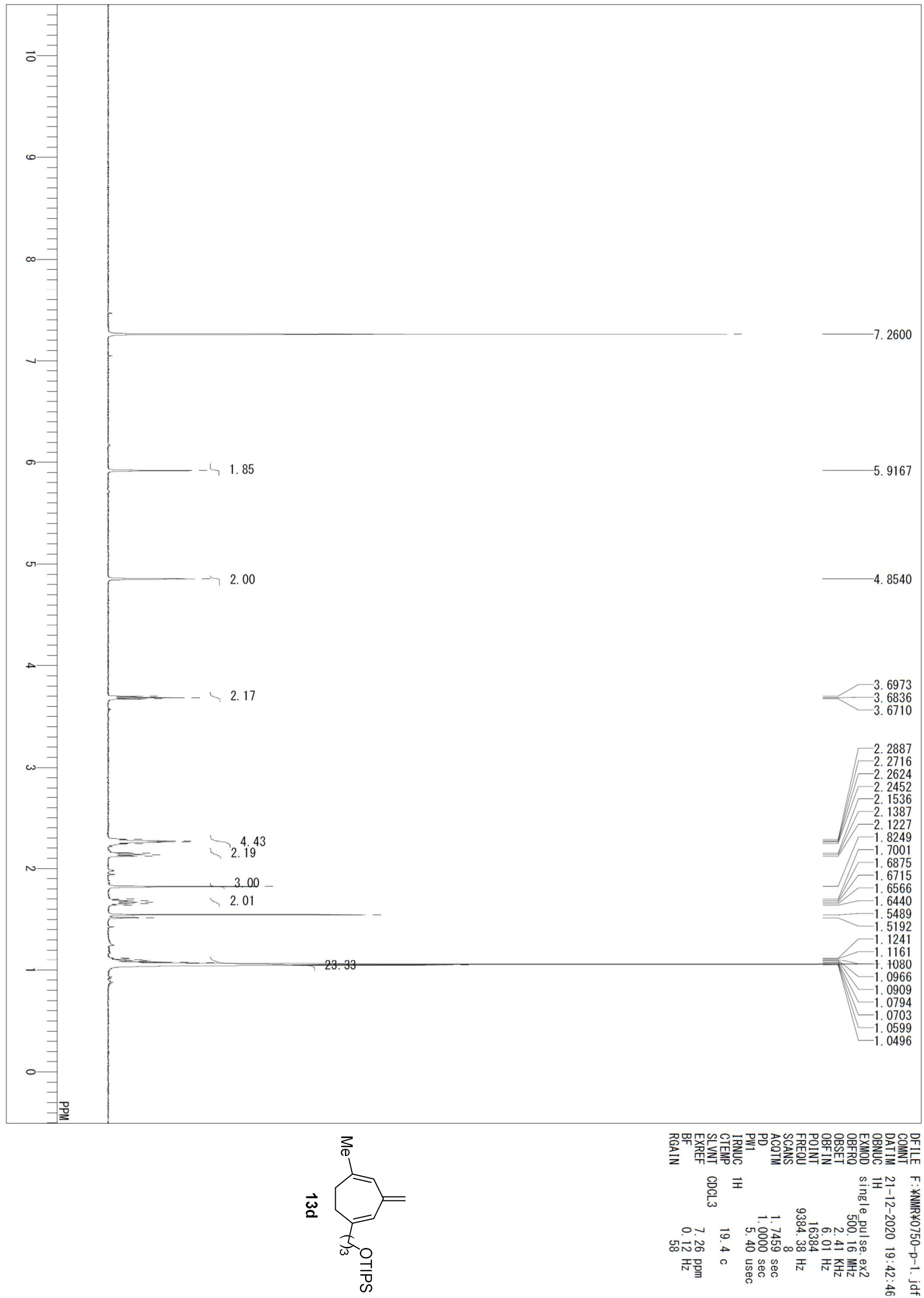

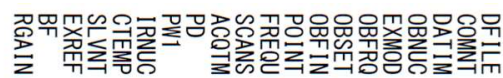

욜

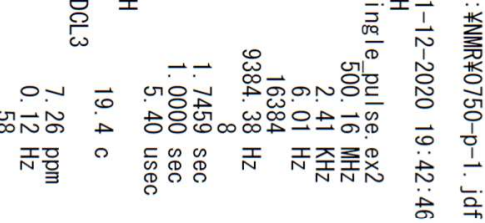




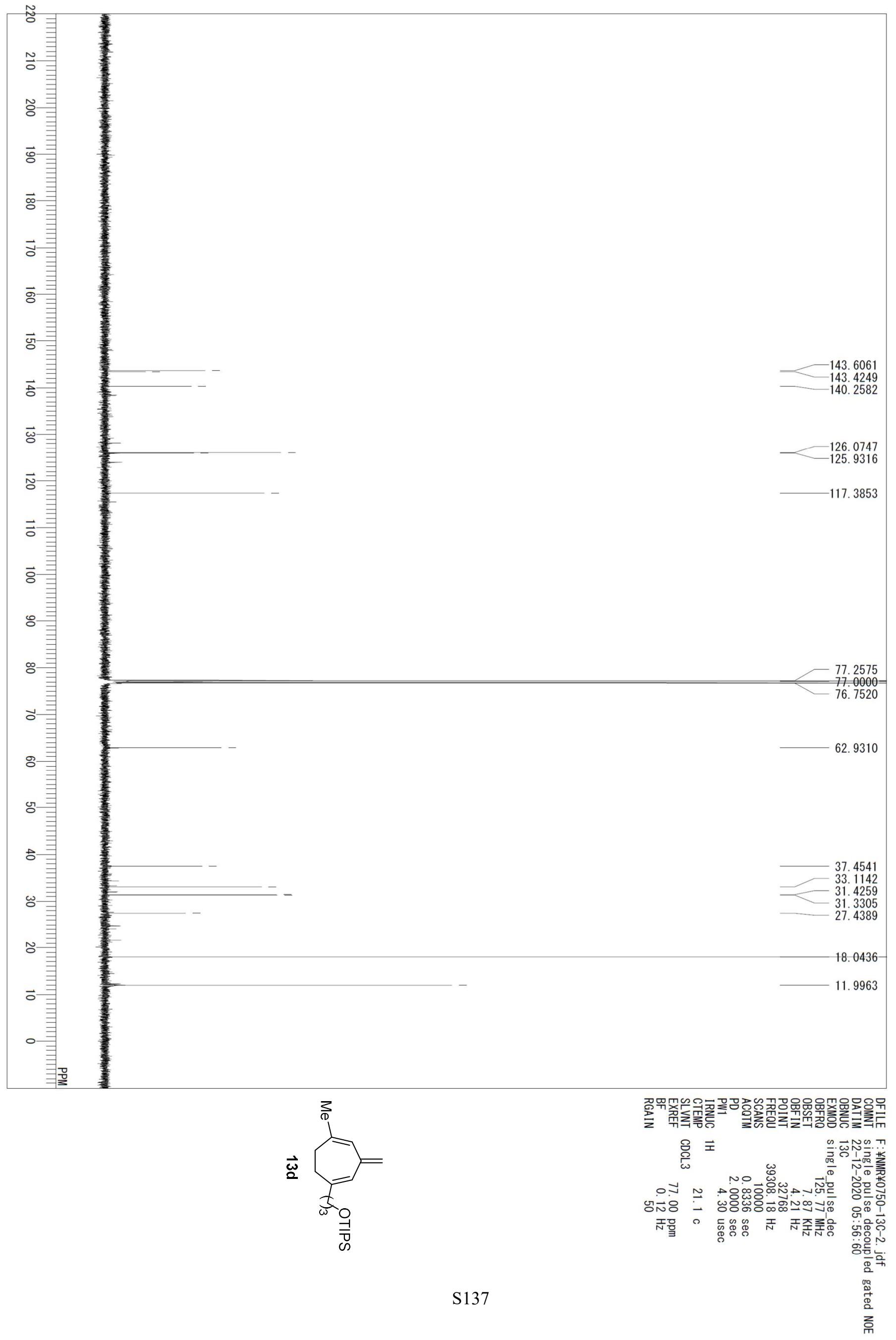



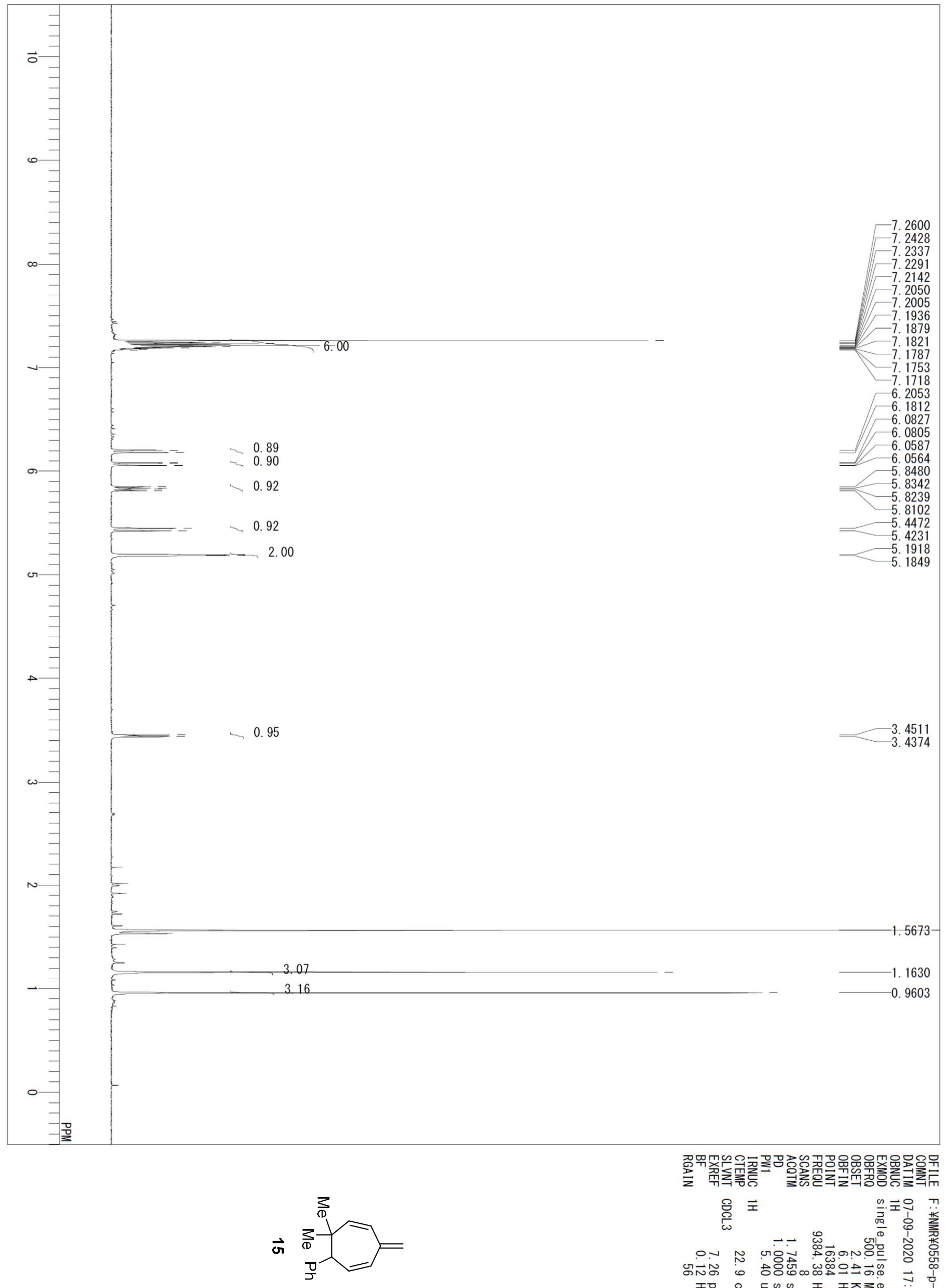

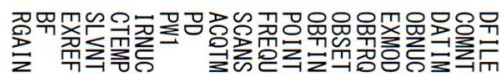

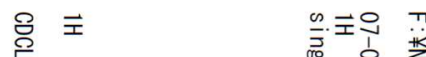

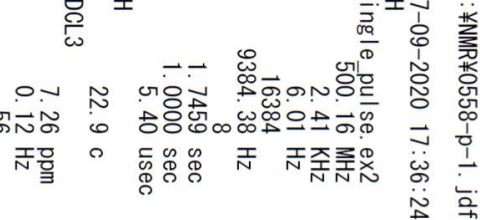



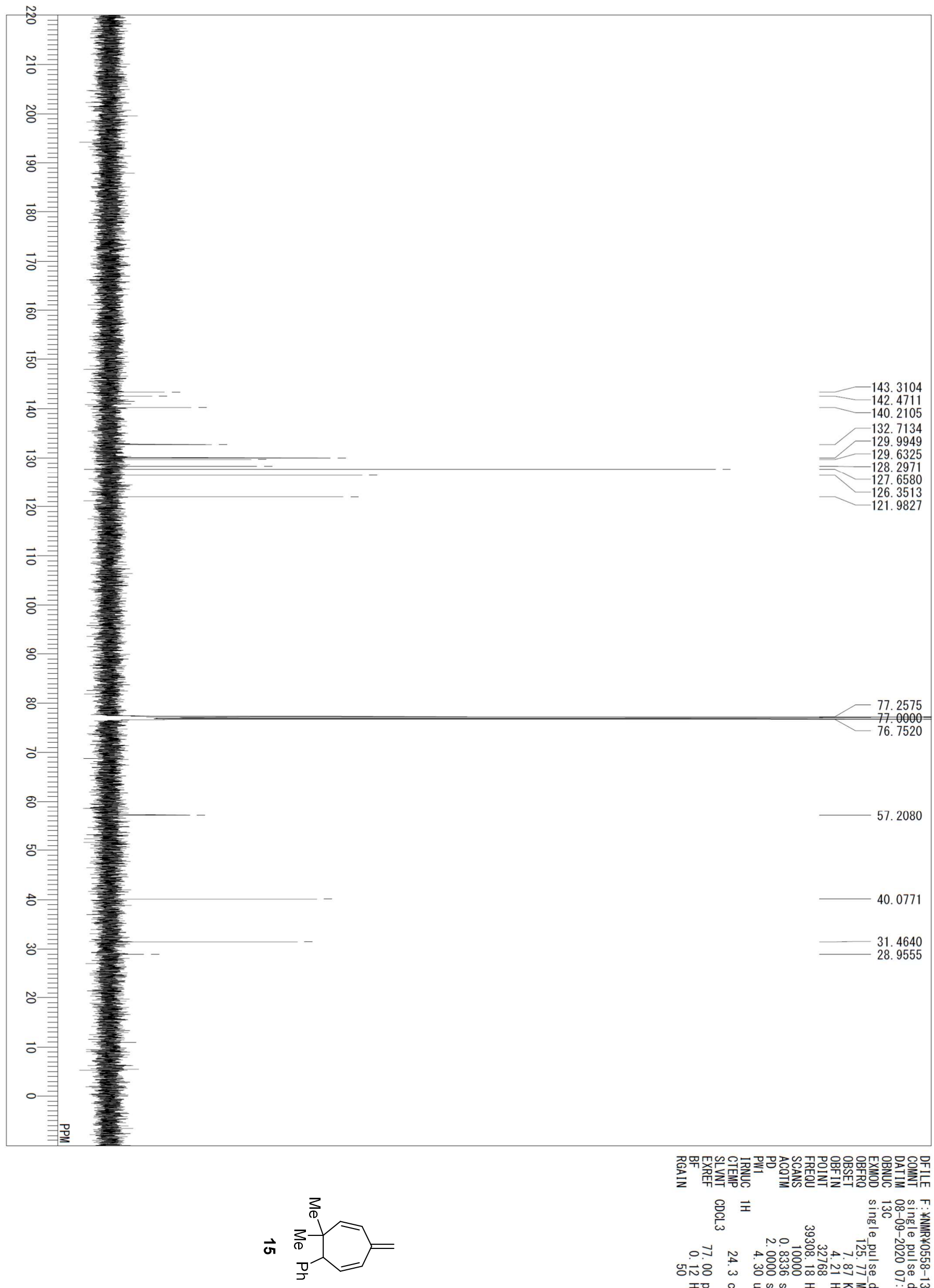

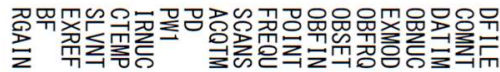
욤표

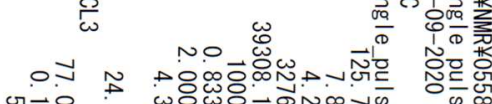

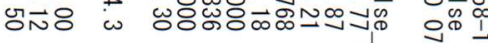

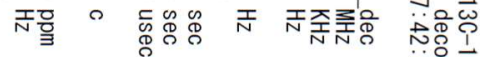
드음 


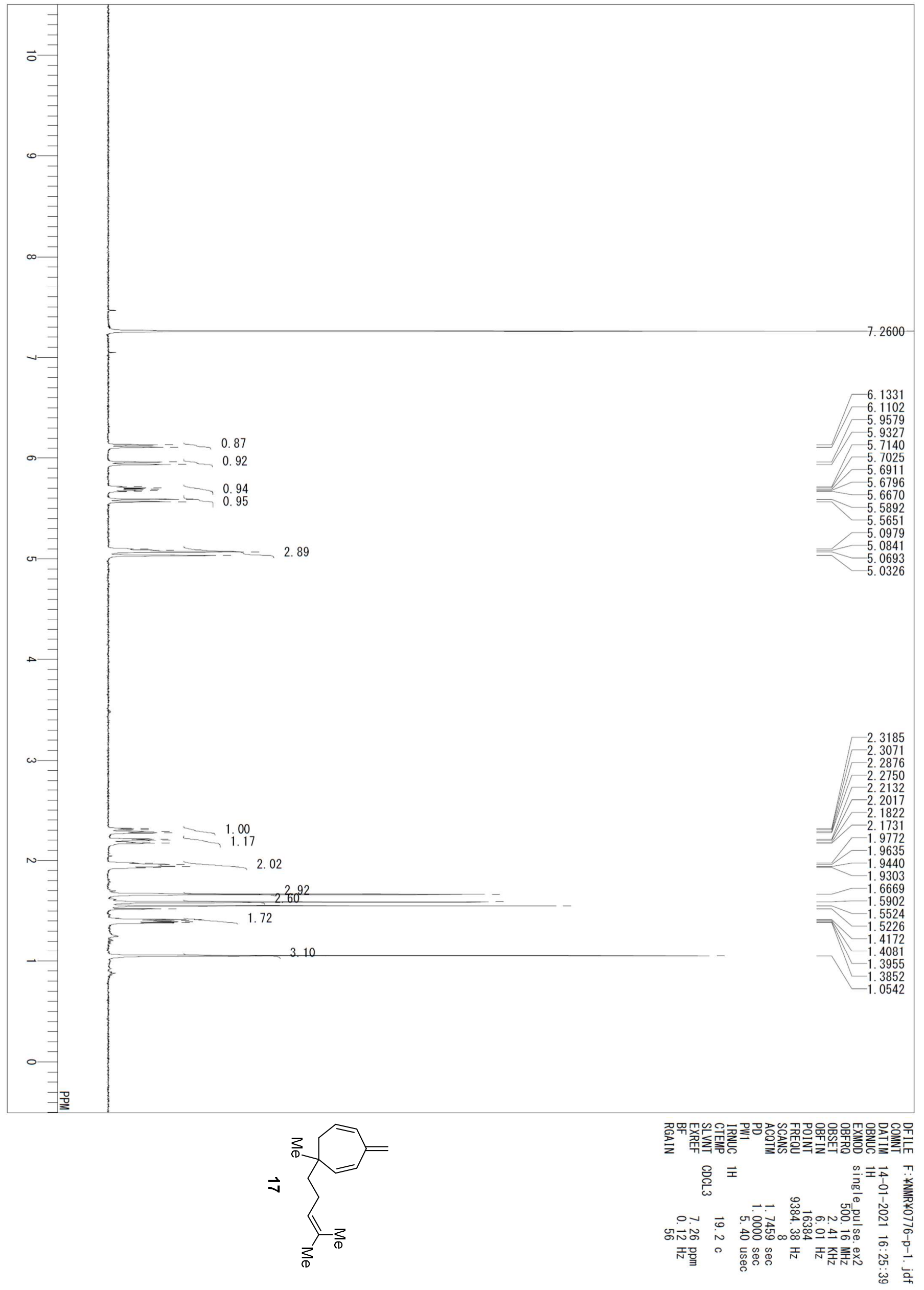




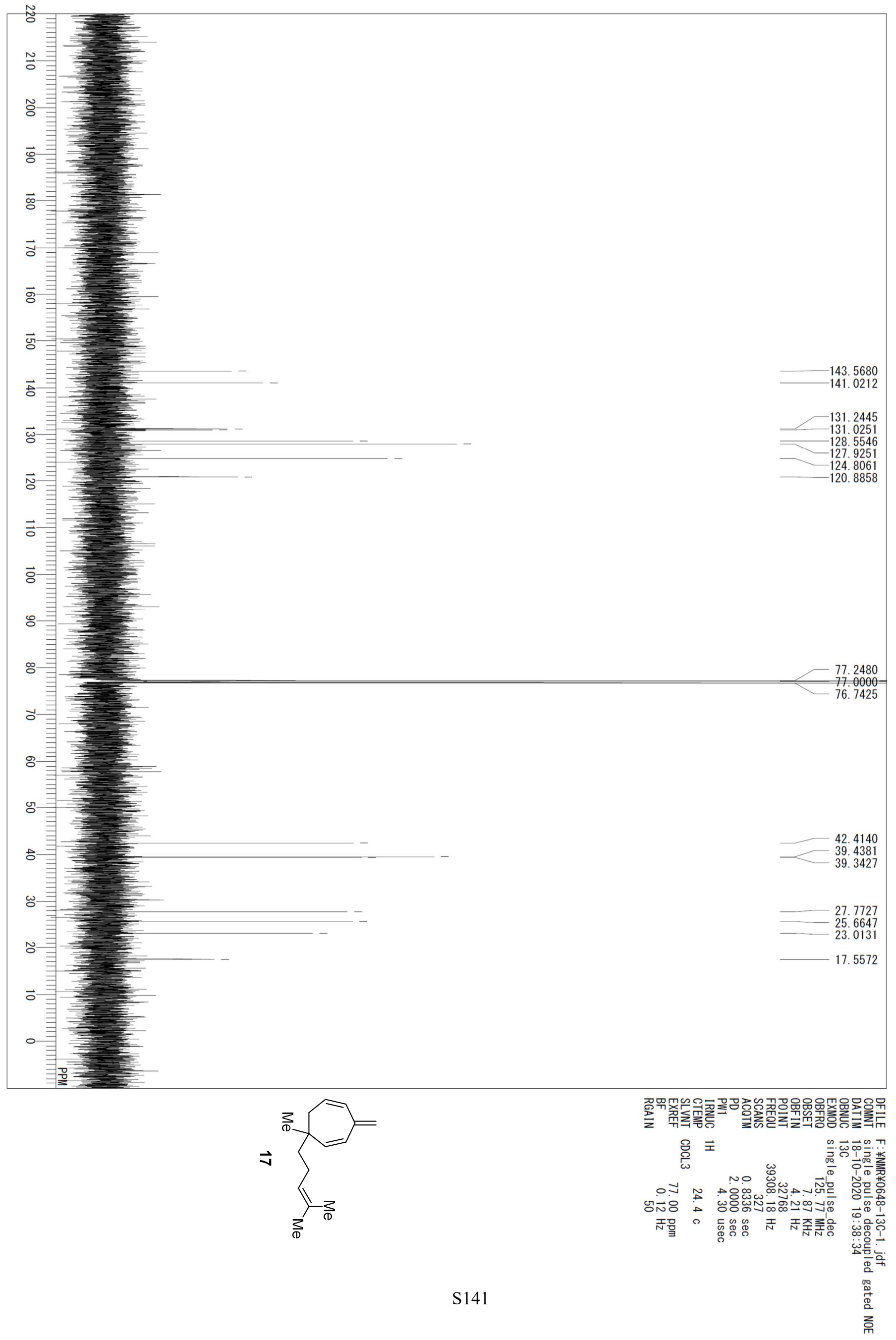



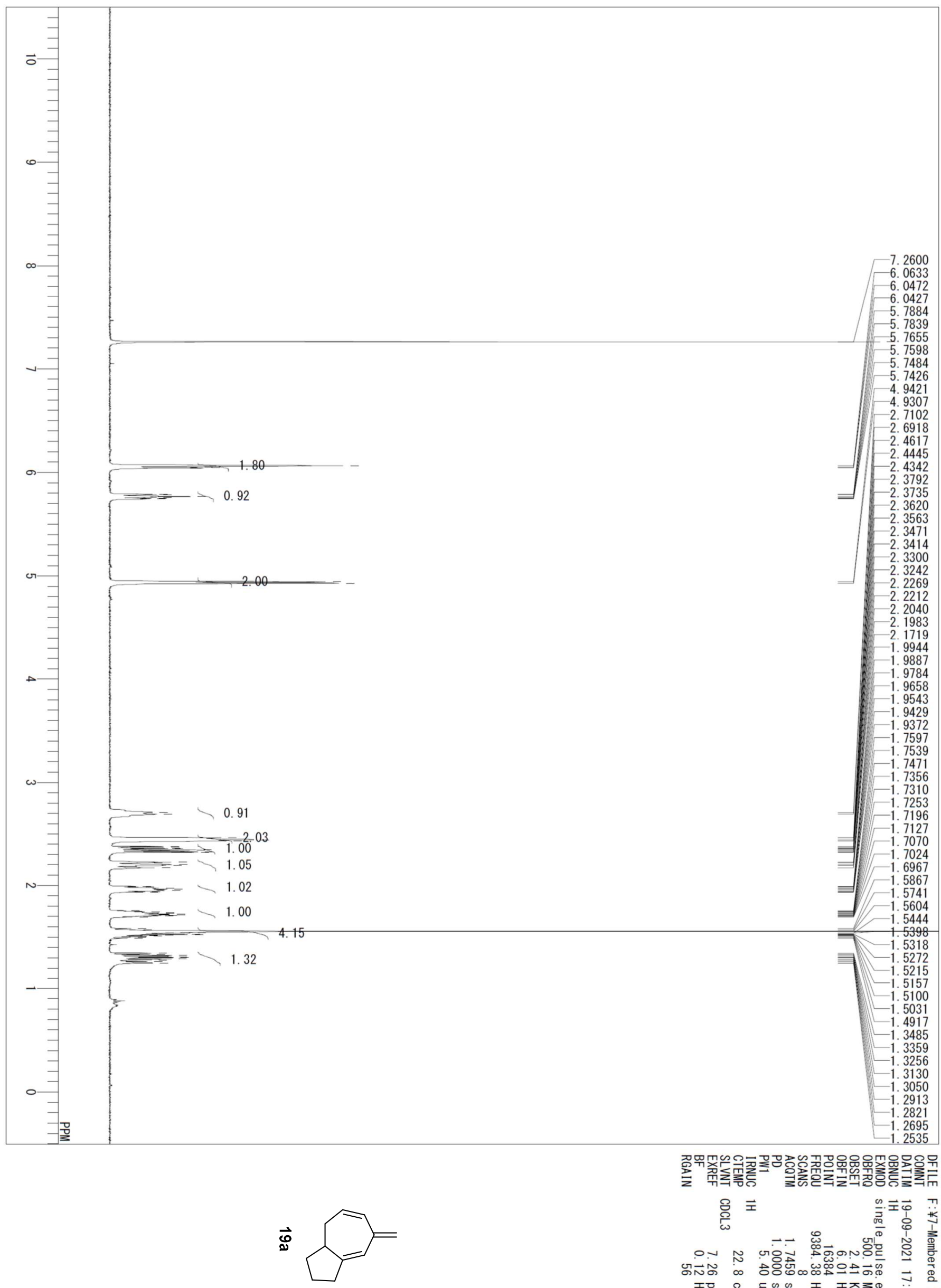

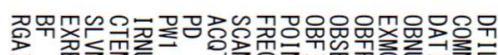

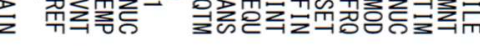

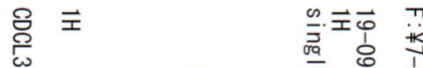

ON N ज施

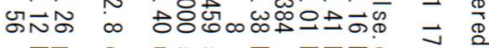

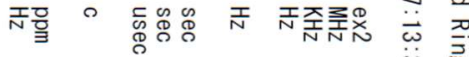




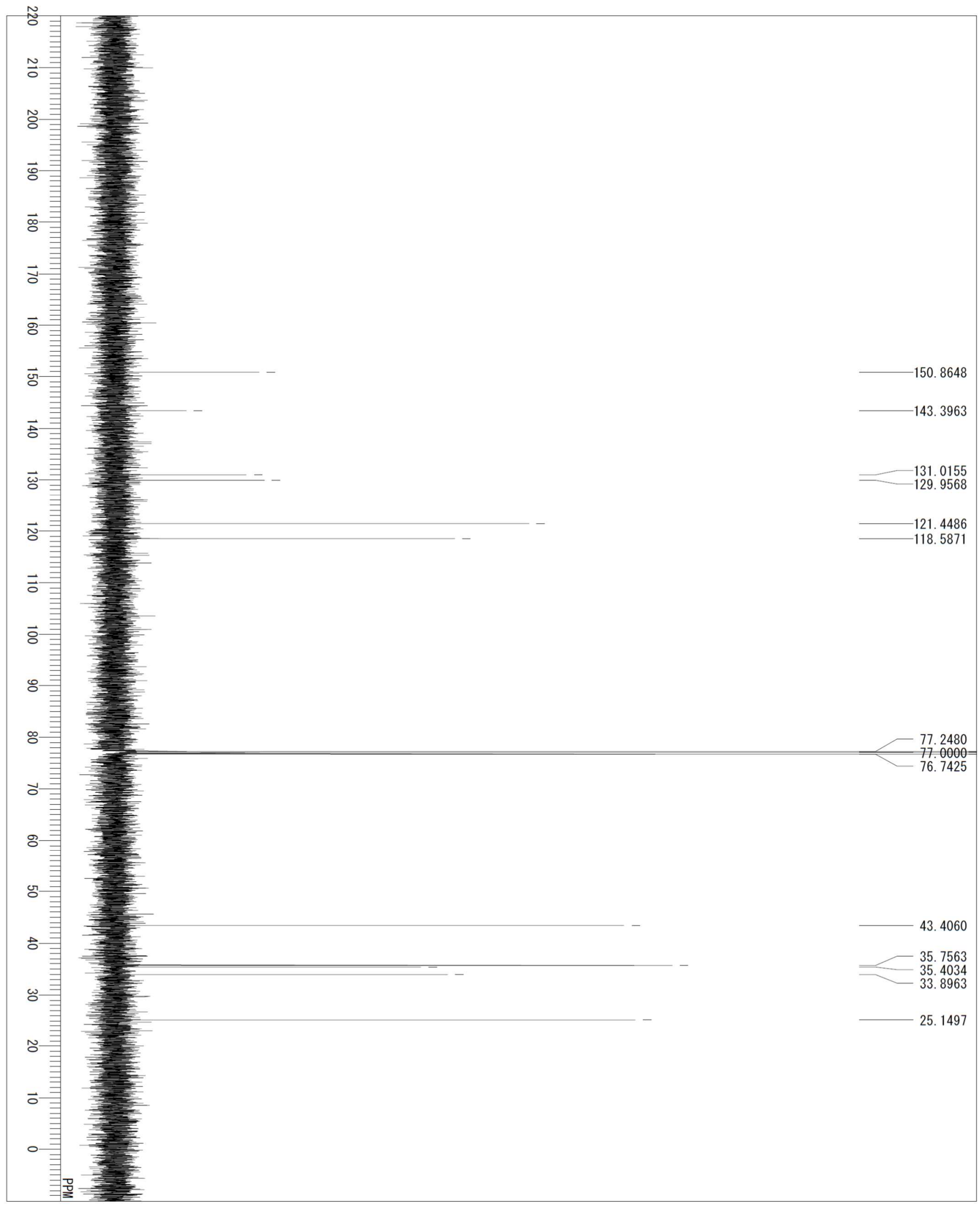

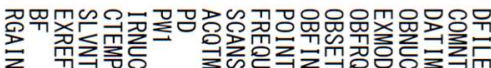<smiles>C1=CC2CCCC(C1)C2</smiles>

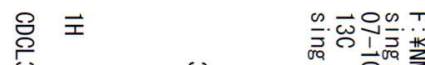

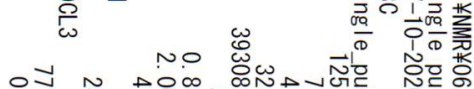

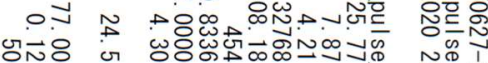

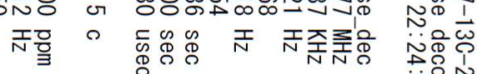



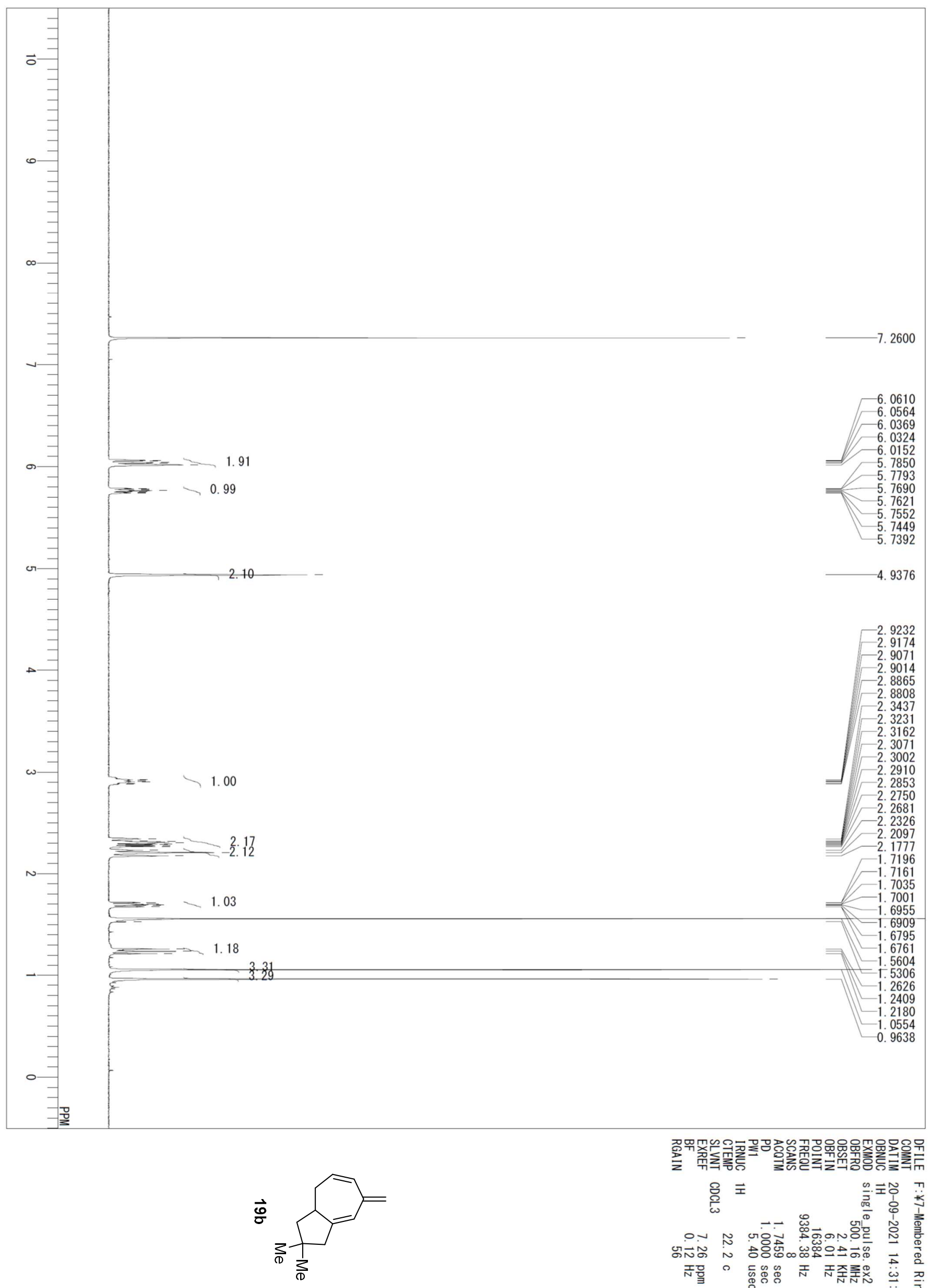

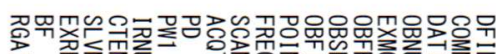

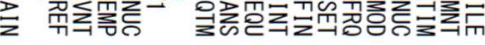

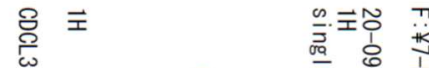

ON N

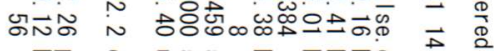

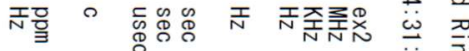

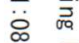



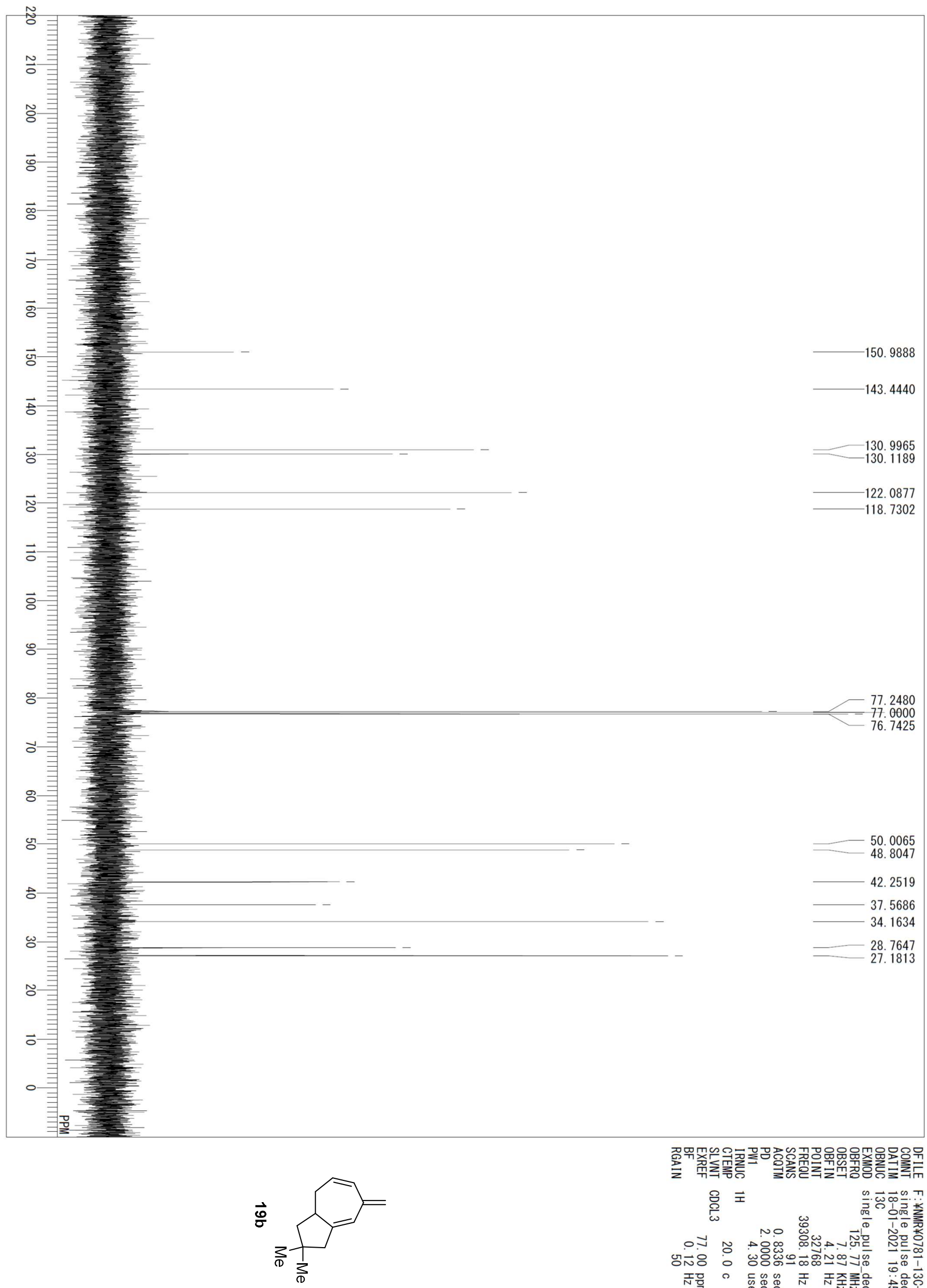

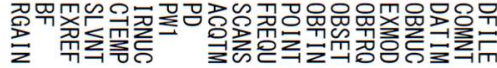

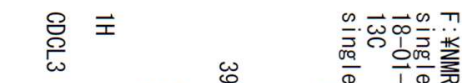

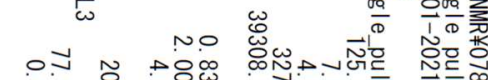

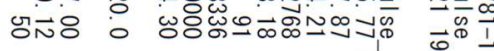

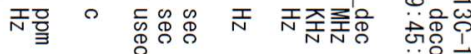




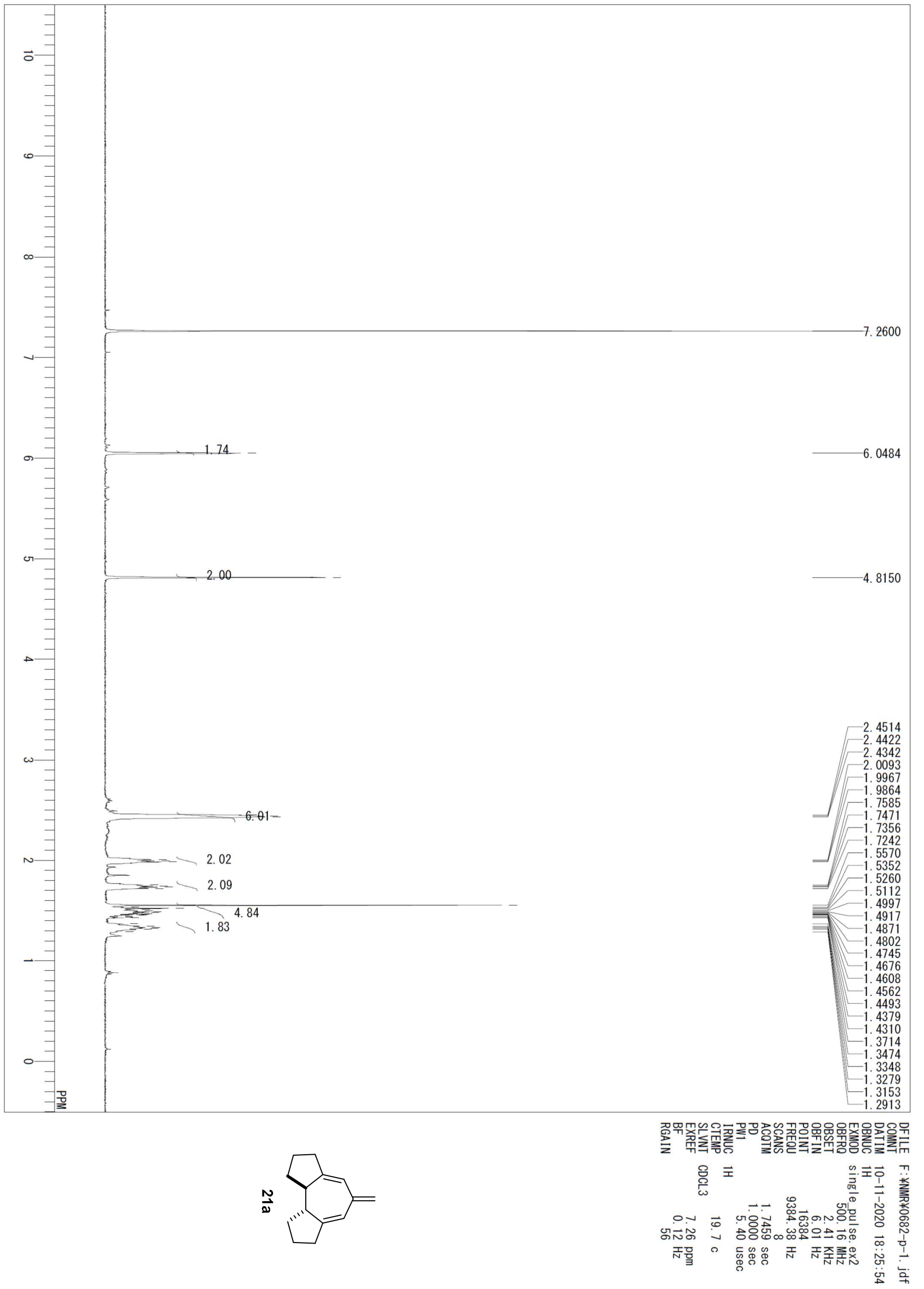



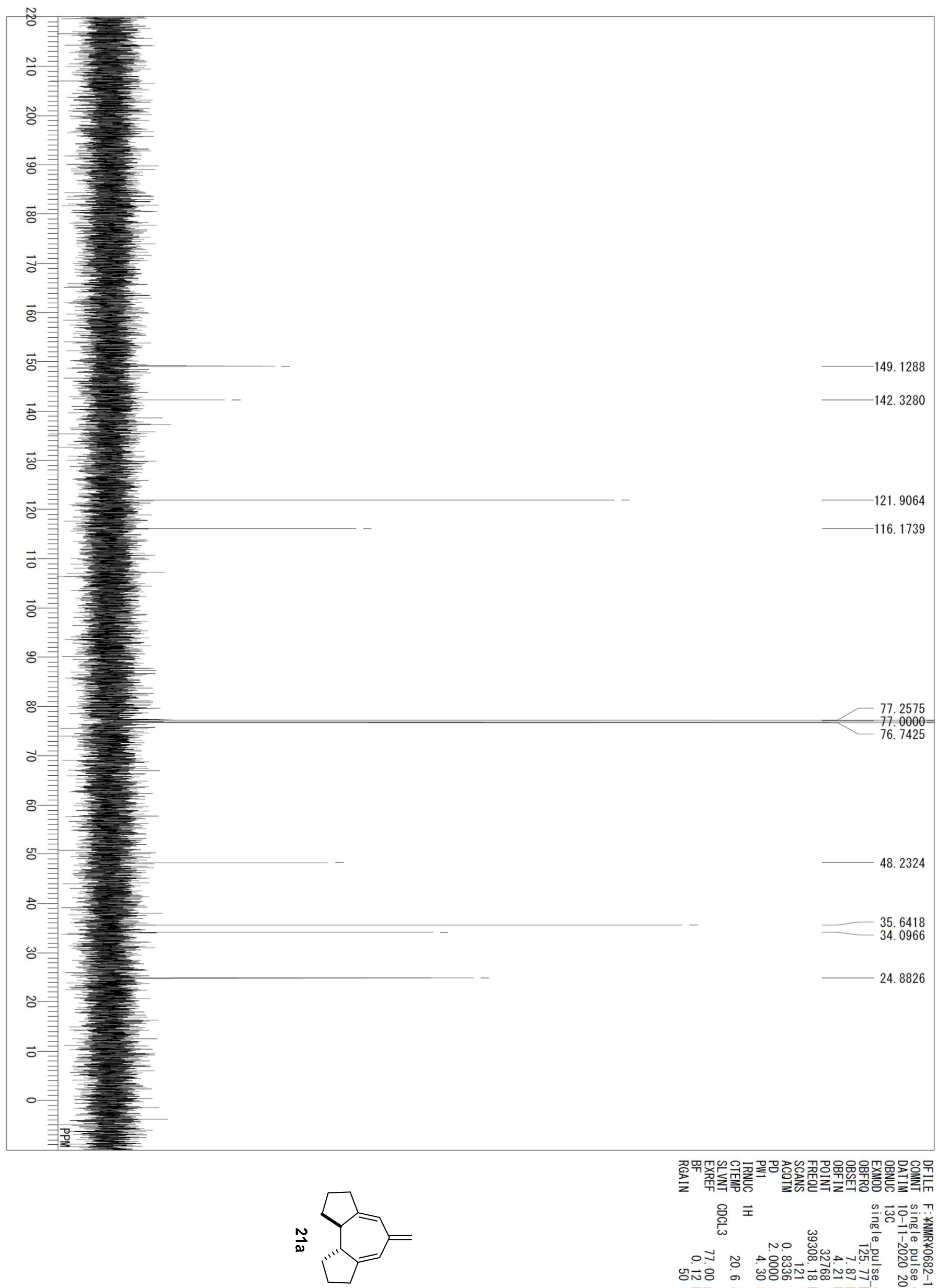

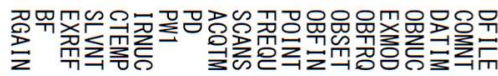

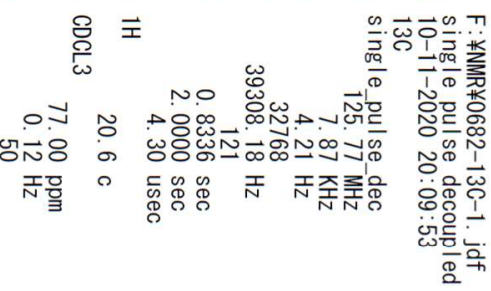




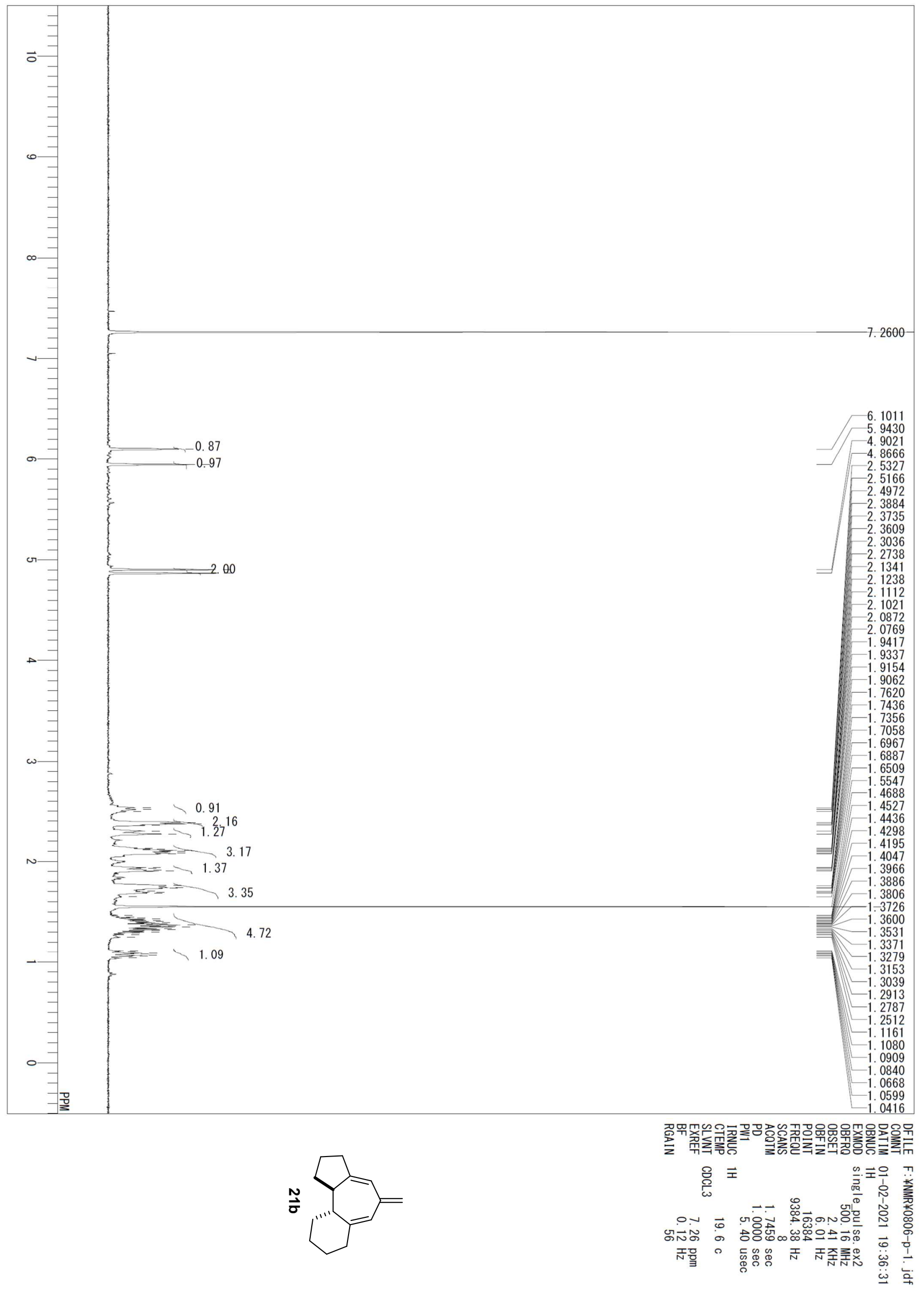



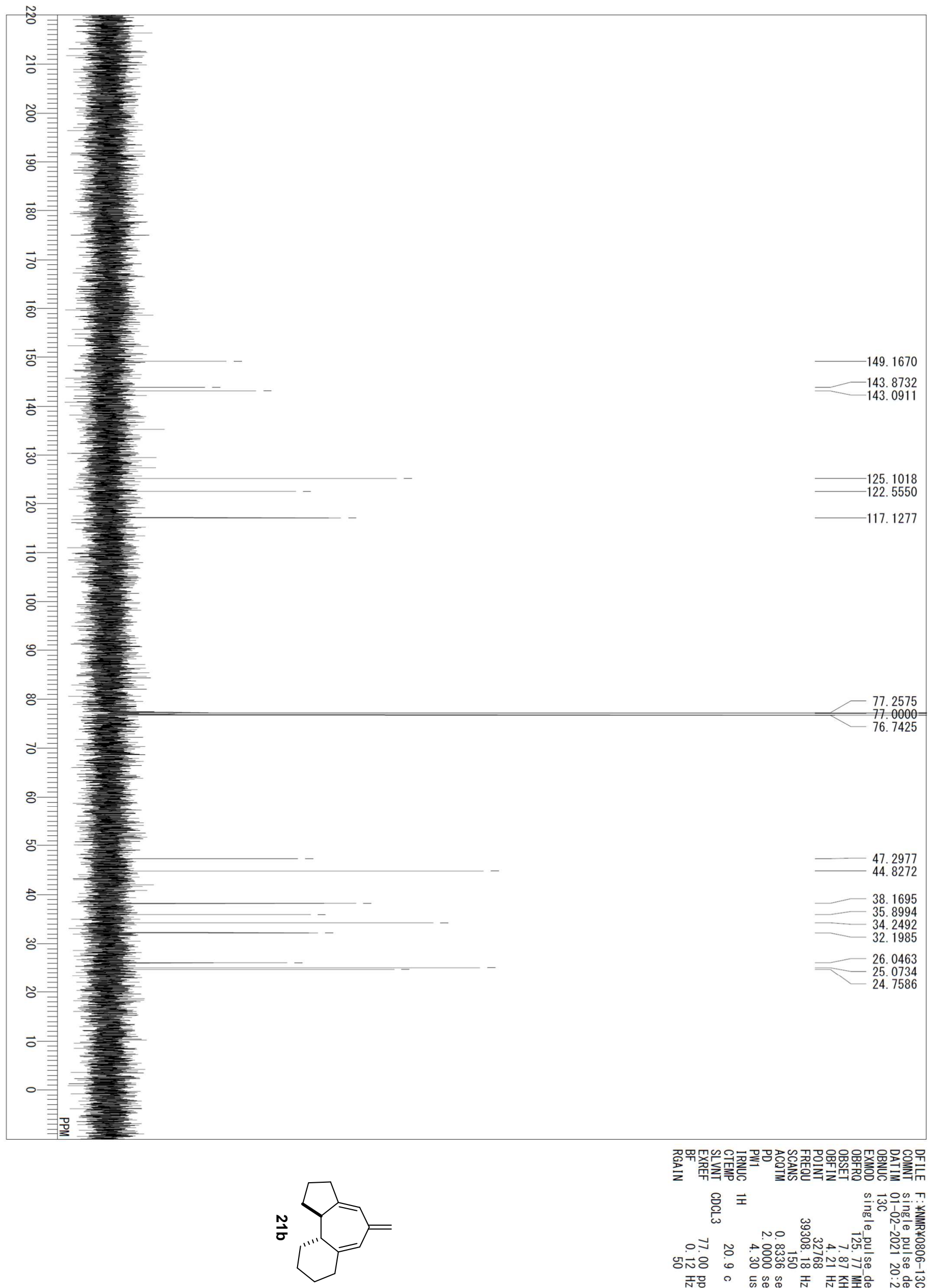

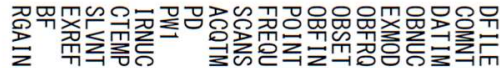

을 궁ำ 귱

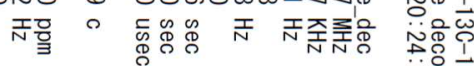



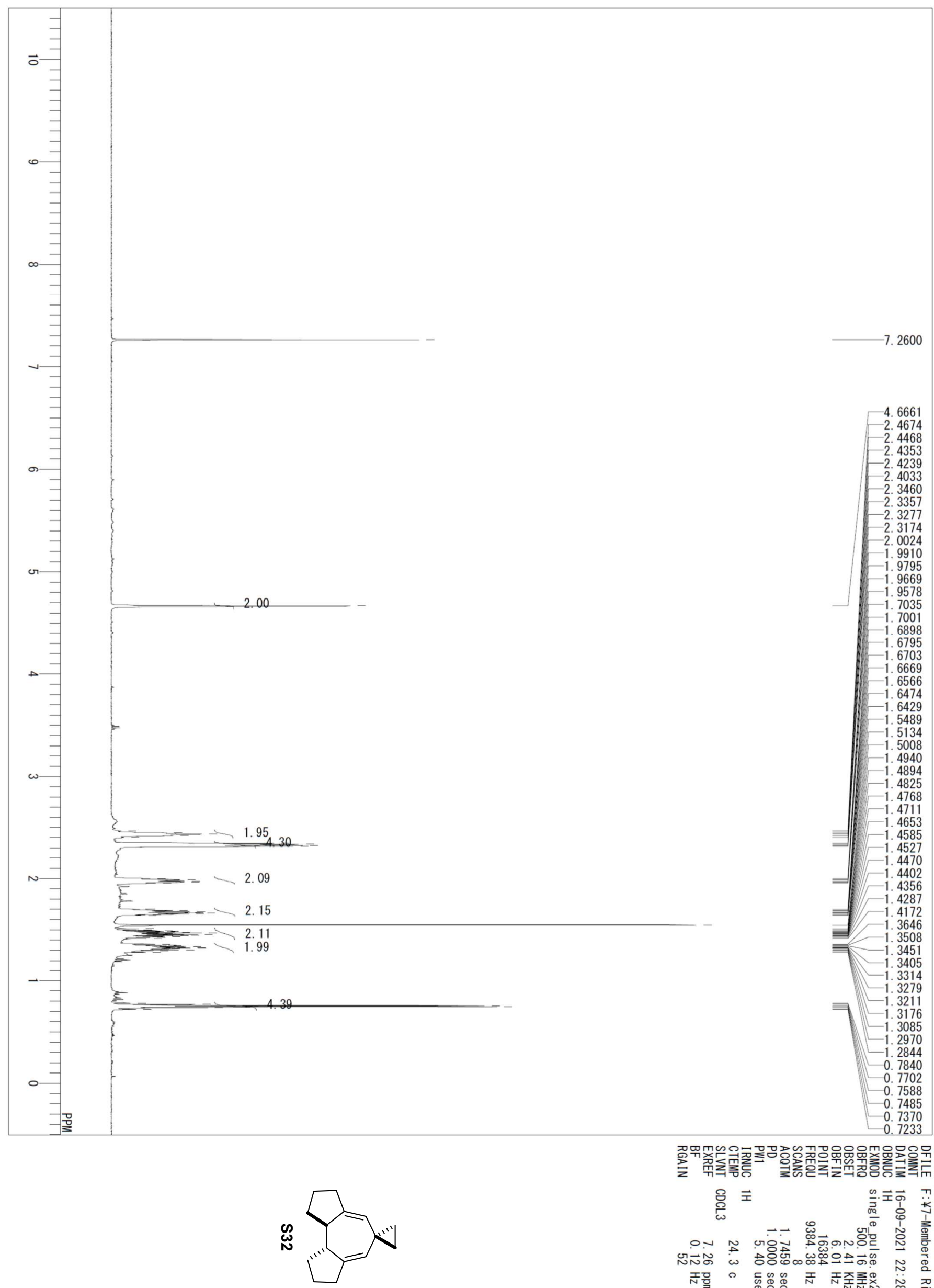

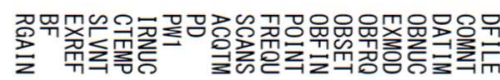

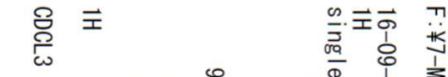

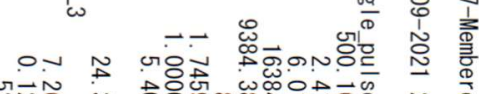

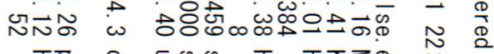

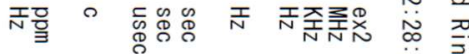
i 


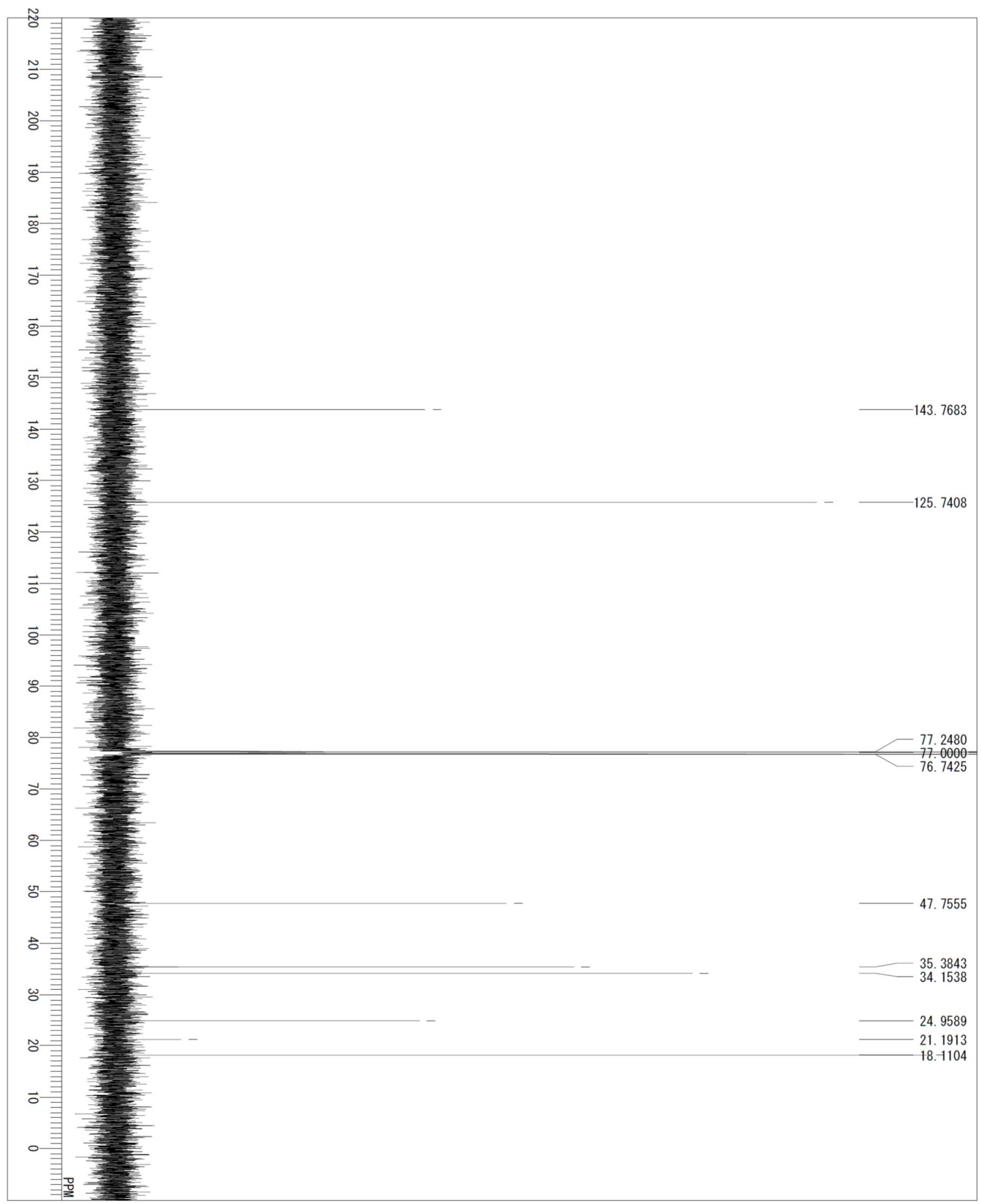

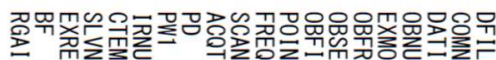

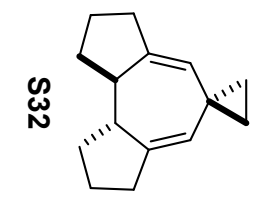

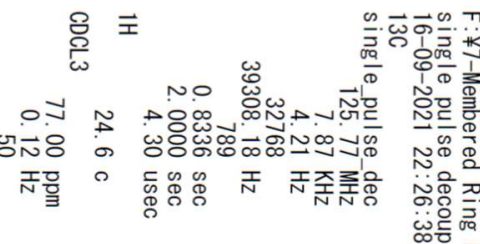




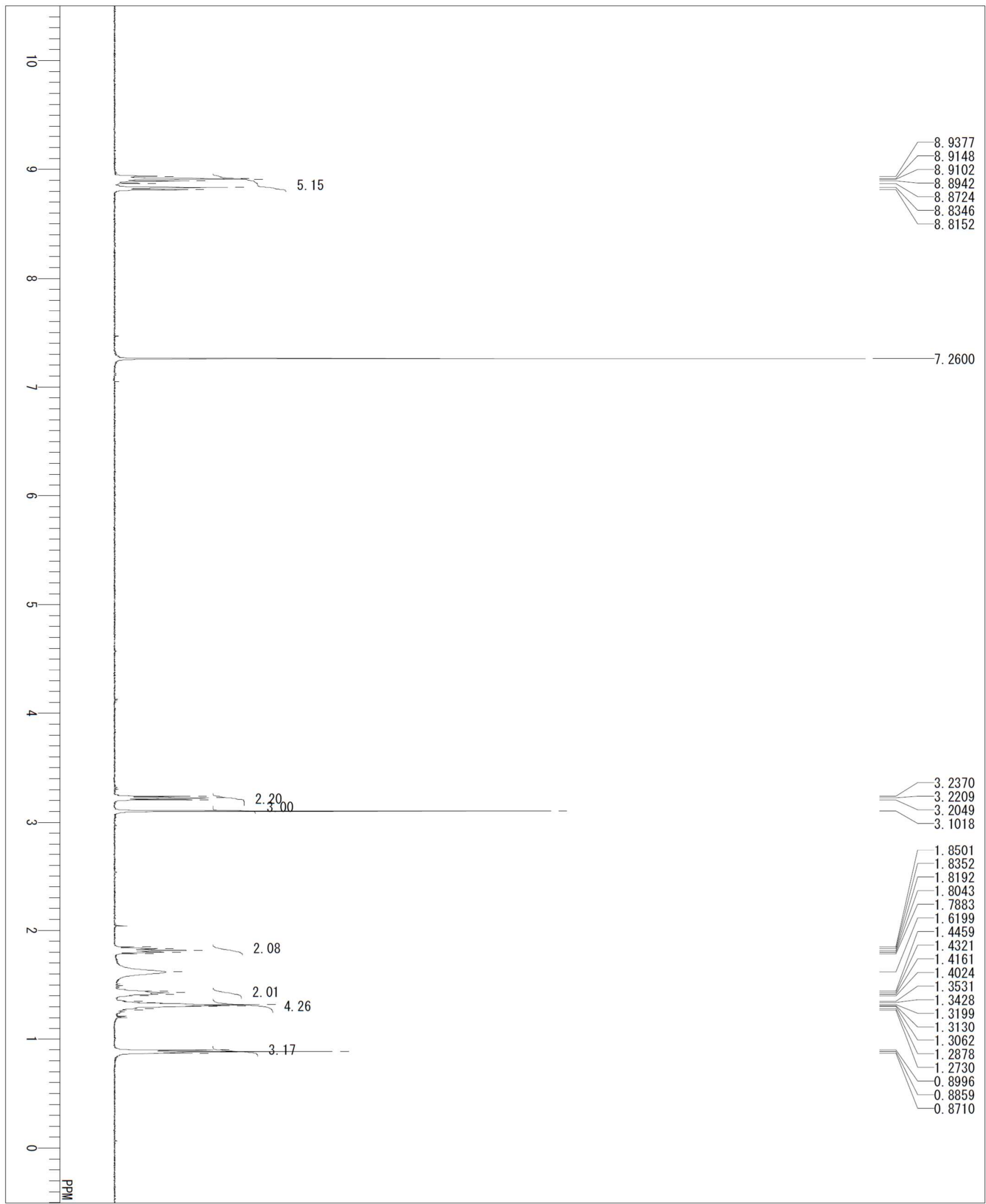

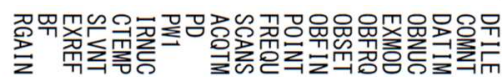

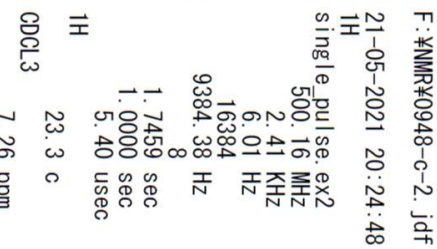




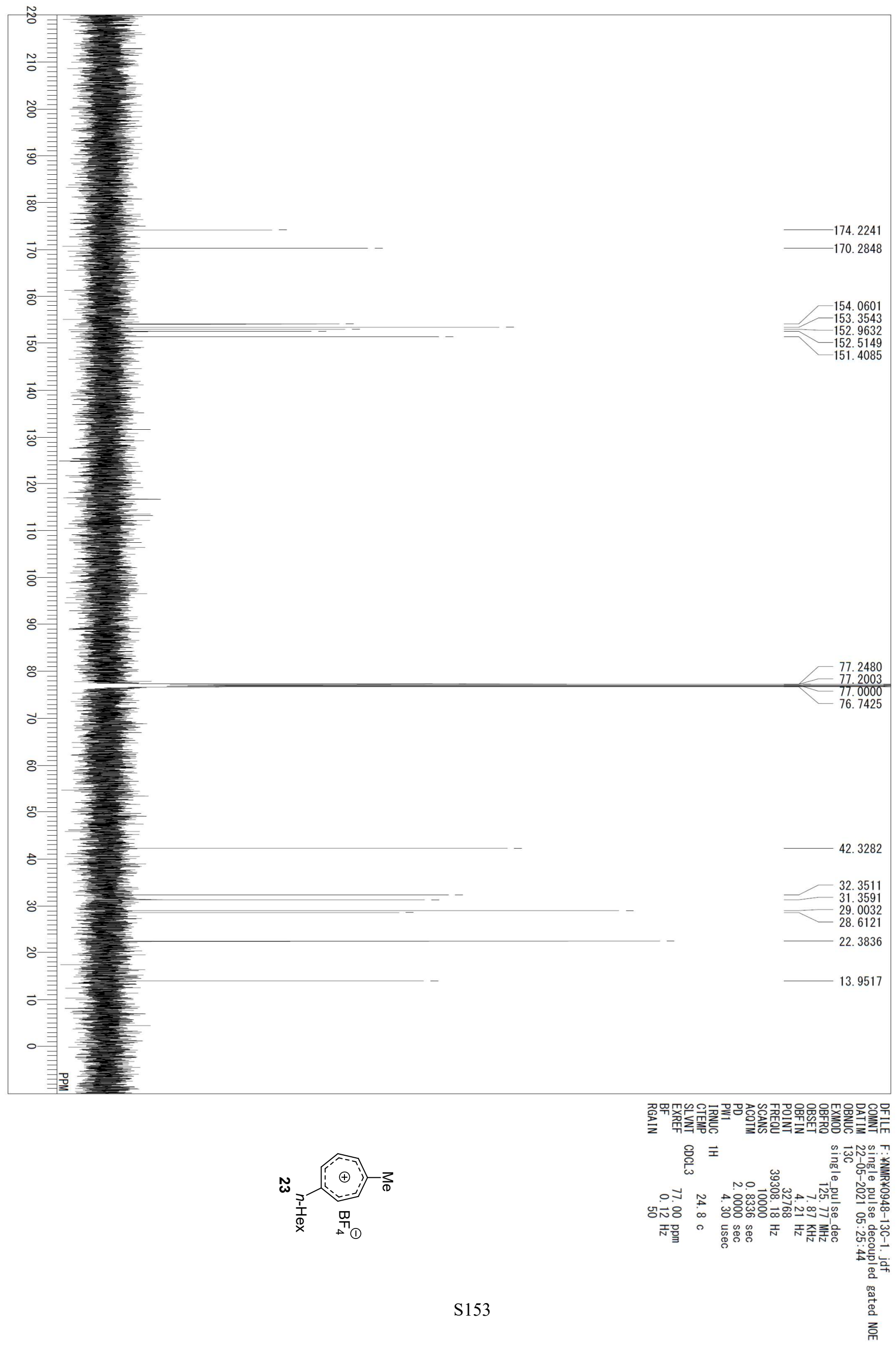


